\title{
Cooperation, Social Selection, and Language Change: An Experimental Investigation of Language Divergence
}

\author{
Gareth Roberts
}

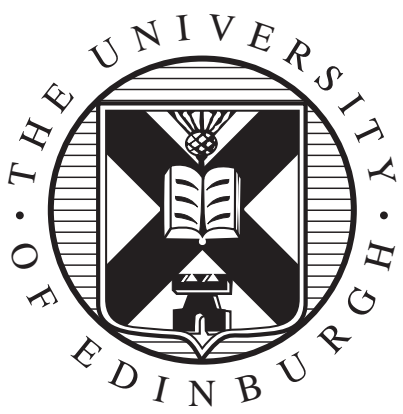

A thesis submitted in fulfilment of requirements for the degree of Doctor of Philosophy

to

Linguistics and English Language

School of Philosophy, Psychology and Language Sciences University of Edinburgh 



\section{Declaration}

I hereby declare that this thesis is of my own composition, and that it contains no material previously submitted for the award of any other degree. The work reported in this thesis has been executed by myself, except where due acknowledgement is made in the text.

Gareth Roberts 



\section{Abstract}

In this thesis, I use an experimental model to investigate the role of social pressures in stimulating language divergence.

Research into the evolution of cooperation has emphasised the usefulness of ingroup markers for swiftly identifying outsiders, who pose a threat to cooperative networks. Mechanisms for avoiding cheats and freeriders, which tend to rely on reputation, or on (explicit and implicit) contracts between individuals, are considerably less effective against short-term visitors. Outsiders, moreover, may behave according to different social norms, which may adversely affect cooperative interactions with them. There are many sources of markers by which insiders and outsiders can be distinguished, but language is a particularly impressive one.

If human beings exploit linguistic variation for this purpose, we might expect the exploitation to have an influence on the cultural evolution of language, and to be involved in language divergence, since it introduces a selective pressure, by which linguistic variants are selected on the basis of their social significance. However, there is also a neutral, mechanistic model of dialect formation that relies on unconscious accommodation between interlocutors, coupled with variation in the frequency of interaction, to account for divergence. In studies of real-world communities, these factors are difficult to tease apart.

The model described in this thesis put real speakers in the artificial environment of a computer game. A game consisted of a series of rounds in which players were paired up with each other in a pseudo-random order. During a round, pairs of players exchanged typed messages in a highly restricted artificial "alien language". Each player began the game with a certain number of points, distributed between various resources, and the purpose of sending messages was to negotiate to exchange these resources. Any points given away were worth double to the receiver, so, by exchanging resources, players could accumulate points for their team. However, the pairings were anonymous: until the end of a round, players were not told who they had been paired with.

This basic paradigm allowed the investigation of the major factors influencing language divergence, as well as the small-scale individual strategies that contribute to it. Two major factors were manipulated: frequency of interaction and competitiveness. In one condition, all players in a game were working together; in another 
condition, players were put into teams, such that giving away resources to teammates was advantageous, but giving them to opponents was not. This put a pressure on players to use variation in the alien language to mark identity. A combination of this pressure and a minimum level of interaction between teammates was found to be sufficient for the alien language to diverge into "dialects". Neither factor was sufficient on its own.

The results of these experiments suggest that a pressure for the socially based selection of linguistic variants can lead to divergence in a very short time, given sufficient levels of interaction between members of a group. 


\section{Acknowledgements}

As an undergraduate, I remember thinking that to list in the bibliography only those authors I had cited directly felt like cheating: surely I should also acknowledge those authors I hadn't mentioned, but whose work had nonetheless influenced me? The danger of such thinking lies in its logical conclusion. No doubt this thesis would not have been quite the same without the various introductory textbooks that helped shape my understanding of the field, many of which are not cited here. But if I were to cite those books on that basis, I should probably cite Tolkien too. I read The Lord of the Rings when I was still in primary school, and I noticed more recently that Appendix F explains the divergence of the Black Speech of the Orcs into a myriad of "barbarous dialects" in terms of social selection (if not in quite those words). A list of all the works that influenced me in creating this thesis would make it a hard task to bind it in one volume, and the same can be said for acknowledgements: there are many people who helped make me the man who wrote this, but who cannot be mentioned by name. You may recognise yourself in that shapeless mass; if so, please accept my thanks.

And now for the names:

My supervisors, April McMahon and Andrew Smith, first accepted my idea for an experiment with just the right mix of caution and enthusiasm. At no point did their enthusiasm wane, and much of what is good about this thesis is due to their advice and suggestions. The experiment might never have got off the ground, however, if I hadn't made a deal with Justin Quillinan that if he'd help me with the programming, I'd help him with his German. It's entirely due to him that the program worked, so I guess it's due to me that his German's still rather shaky. Sorry, mate.

Thanks are further due...

Academically, to Dan Dediu, Alexandra Dima, Frances Wilson, and Francesca Filiaci for their invaluable advice on statistics; to Martin Pickering, Thomas Bąk, Jamie Tehrani, Nikolaus Ritt, and Emma Cohen for their encouraging interest in my work and for several insightful questions and suggestions; to Mike Bennett, Eddie Dubourg, Cedric MacMartin, and Morag Brown for their technical support; to Katie Keltie, Toni Noble, Lynsey Buchanan, Stephanie Fong, and Devon Hawthorn for their administrative support and the lunchtime quizzes; to Rob Clark for having a perception lab when I needed one in an emergency, and his helpfulness and efficiency in giving me access to it; to everyone in the LEC, and in LEL more broadly, for 
the research environment they helped create-I can't help fearing I've just been spectacularly lucky and will never work in such a nice one again. And to Simon Kirby and Simon Garrod, who managed to make my viva far more enjoyable than I imagined it could be.

Slightly less academically, I'm grateful to Cyprian Laskowsi, Hannah Cornish, Lauren Stewart, Will Barras, Remco Knooihuizen, Karen Ludke, and Derek Murphy for being good friends, good colleagues, and good guinea pigs on early pilots of my experiment; to the lunch crowd, including Peter Bell, Dan Wedgewood, Lynda Hutchison, and quite a few other people already mentioned above; to Sebastian Lopez, Amy Wallace, Donal Mulvihill, and Azita Ayatinejad for being prepared to live with me; to Jill Bates, Hae Sung Jeon, Patrick Power, and Etta Gran Myreng who were there when I started in Edinburgh and who kept in touch from all the different places they moved to; to Peter Methven, even though it was me who moved away; to Jenny Graver for a very long and enjoyable email conversation about linguistics, books, and music; to Tom Roberts, Jo O'Hara, and Zoë Payne for weekly pub quizzes throughout my final year, and to David Harris who won them for us. It is due to all these people that I never found my PhD isolating.

Finally there's my family. I am grateful first to my parents for more things than I can list. I'll mention two: the financial help that saw me through to the end, and their unwavering pride in me. I hope they know I'm equally proud of them. Second, there are two individuals who weren't even in my life when I started my PhD: Ksyusha, who has been welcoming me home every day for the last year by rolling on the carpet and inviting me to rub her belly; and Lottie, who hasn't, but who has done more for me and who means more to me than I can ever thank her for. Thank you all the same. 


\section{Contents}

Declaration

Abstract

iii

Acknowledgements

$\mathrm{V}$

List of Tables

$\mathrm{XV}$

List of Figures

xvii

Chapter 1 Introduction 1

1.1 Why suicide bombers shouldn't have Yorkshire accents . . . . . . . 1

1.2 The drive to sound different . . . . . . . . . . . . . . . . . 3

1.3 An underused methodology . . . . . . . . . . . . . . . 4

1.4 Other questions ...................... 5

$\begin{array}{lll}\text { Chapter } 2 & \text { The problem of cooperation } & 7\end{array}$

2.1 Defining cooperation . . . . . . . . . . . . 7

2.2 Explaining cooperation . . . . . . . . . . . . . . . . . . 10

2.2.1 Indirect benefits and altruistic behaviour . . . . . . . . . . . 11

2.2.1.1 Mechanisms of kin recognition . . . . . . . . . 12

2.2.2 Enforcement in mutually beneficial behaviour . . . . . . . . 14

2.3 The problem of freeriders . . . . . . . . . . . . . . 16

2.4 Identifying oneself and others . . . . . . . . . . . . . . . . . 17

2.4.1 Markers ...................... 19

2.4.2 Criteria for good markers . . . . . . . . . . 20

2.4.2.1 Broader explanations for cultural markers . . . . . 23 
2.4.3 Social selection . . . . . . . . . . . . . . 25

$\begin{array}{lll}\text { Chapter } 3 & \text { Change and variation in language } & 27\end{array}$

3.1 The meaning of variation and change in language . . . . . . . . 27

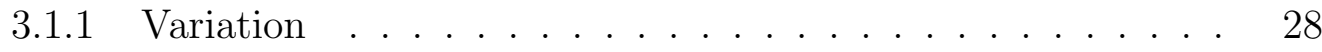

3.1 .2 Change ........................ 30

3.1.2.1 Change and diachronic variation . . . . . . . 33

3.1.2.2 Innovation and propagation . . . . . . . . 34

3.1.2.3 Production and competence . . . . . . . . . . 34

3.1.2.4 Kinds of change . . . . . . . . . . . . . . . 36

3.2 Convergence and divergence . . . . . . . . . . . . . . . . 36

3.3 Social factors in language change . . . . . . . . . . . . . . 38

3.3.1 Trudgill and new-dialect formation . . . . . . . . . . . 40

3.3.1.1 Claims and criticisms . . . . . . . . . . . 42

3.3.1.2 Accommodation and alignment . . . . . . . . 46

3.4 Conclusion . . . . . . . . . . . . . . . . . . . . . . . . 48

$\begin{array}{lll}\text { Chapter } 4 & \text { Linguistic variants in cultural-evolutionary models } & 51\end{array}$

4.1 Language as an adaptive system . . . . . . . . . . . . . . . . . . 51

4.2 Memetic models of language . . . . . . . . . . . . . . . . . . 55

4.2.1 Ritt and neural assemblies . . . . . . . . . . . . . 56

4.2 .2 Croft and utterances . . . . . . . . . . . . . 58

4.2.3 Candidates for linguistic replicators . . . . . . . . . . . . 61

4.2.3.1 Background conditions . . . . . . . . . . . 62

4.2.3.2 Phonetic tokens as a special case . . . . . . 65

4.3 Conclusion . . . . . . . . . . . . . . . . . . . 68

$\begin{array}{lll}\text { Chapter } 5 & \text { Testing the hypothesis: a first experiment } & 71\end{array}$

5.1 Methods in historical linguistics . . . . . . . . . . . . . . . 71

5.2 Description of experiment . . . . . . . . . . . . . . 75

5.2 .1 Overview ....................... 75

5.2.1.1 A typical round . . . . . . . . . . . 76

5.2 .1 .2 Research questions . . . . . . . . . . . 77 
5.2 .2 Participants . . . . . . . . . . . . . . . 77

5.2.3 Experimental procedure . . . . . . . . . . 77

5.2.4 Game structure . . . . . . . . . . . . 80

5.2.5 Resources and scoring . . . . . . . . . . . . . . 82

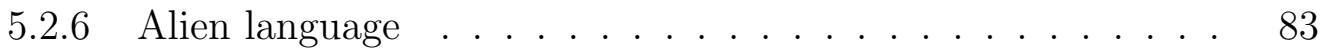

5.3 Summary of terms . . . . . . . . . . . . . . 84

5.4 Results . . . . . . . . . . . . . . . . . 86

5.4 Recognising teammates . . . . . . . . . . . 88

5.4 .2 Divergence . . . . . . . . . . . . . . . . . . . . . . 90

5.4.2.1 Syntactic variation . . . . . . . . . . 93

5.4 .3 Comparison with score . . . . . . . . . . . . . 95

5.5 Strategy . . . . . . . . . . . . . . . . 96

5.5 .1 Identification strategies . . . . . . . . . . . . . 96

5.5.2 Giving behaviour ................... . . 99

5.5 .3 Summary . . . . . . . . . . . . . . . 100

5.6 Discussion ........................... 100

Chapter 6 Controlling for the pressure to mark identity: a second experiment103

6.1 Description of experiment . . . . . . . . . . . . . . . 104

6.1 .1 Overview .......................... 104

6.1 .2 Participants .................... 105

6.1.3 Experimental procedure . . . . . . . . . . . 105

6.1.4 Game structure . . . . . . . . . . . . . . . 106

6.1.5 Resources and scoring . . . . . . . . . . . . 107

6.1.6 Alien language . . . . . . . . . . . . . . . . . . 107

6.1.7 Summary of differences between first and second experiments 108

6.2 Summary of terms . . . . . . . . . . . . . . . . . . . 109

6.3 Results . . . . . . . . . . . . . . . . . . 109

6.3.1 Recognising team-mates . . . . . . . . . . . . . . 110

6.3.1.1 Feedback questionnaires . . . . . . . . . . . 110

6.3.1.2 Explicit and implicit judgements in the Competitive condition .................. 113 
6.3.1.3 Chatting time . . . . . . . . . . . . . . 114

6.3.2 Divergence........................ 115

6.3.2.1 Other sources of divergence . . . . . . . . . 115

6.3 .3 Comparison with score . . . . . . . . . . . 116

6.4 Strategy . . . . . . . . . . . . . . . . . . 116

6.4 .1 Identification strategies . . . . . . . . . . . . . . 116

6.4 .2 Giving behaviour . . . . . . . . . . . . . . 117

6.4 .3 Summary of results . . . . . . . . . . . . . . 118

6.5 Discussion . . . . . . . . . . . . . . . . . 120

$\begin{array}{lll}\text { Chapter } 7 & \text { Raising the frequency of interaction: a third experiment } & 121\end{array}$

7.1 Description of experiment . . . . . . . . . . . . . . . 122

7.1 .1 Overview .......................... 122

7.1.2 Participants . . . . . . . . . . . . . . . 122

7.1.3 Experimental procedure . . . . . . . . . . . . 123

7.1.4 Game structure . . . . . . . . . . . . . . . . 123

7.1.5 Resources and scoring . . . . . . . . . . . . . 123

7.1 .6 Alien language . . . . . . . . . . . . . . . . . . . 123

7.1.7 Summary of differences between experiments . . . . . . . . . 124

7.2 Results . . . . . . . . . . . . . . . . . . . . 124

7.2.1 Recognising team-mates . . . . . . . . . . . 125

7.2.1.1 Feedback questionnaires . . . . . . . . . . 125

7.2.1.2 Explicit and implicit judgements in the Competitive condition . . . . . . . . . . . . . . . . 128

7.2 .2 Divergence. . . . . . . . . . . . . . . . . . 128

7.2.2.1 Other sources of divergence . . . . . . . . . . . 129

7.2 .3 Comparison with score . . . . . . . . . . . . . . . 130

7.3 Strategy . . . . . . . . . . . . . . . . . 130

7.3.1 Identification strategies . . . . . . . . . . . . . 130

7.3 .2 Giving behaviour . . . . . . . . . . . . . . 133

7.3.3 Summary of results . . . . . . . . . . . . . . . . . 134

7.4 Discussion . . . . . . . . . . . . . . . . . . . . 134 
$\begin{array}{lll}\text { Chapter } 8 & \text { Explaining variation in gift size: a fourth experiment } & 137\end{array}$

8.1 Comparing the second and third experiments . . . . . . . . . . . 137

8.2 Description of experiment . . . . . . . . . . . . . . . . . . . 138

8.2.1 Overview ......................... 138

8.2 .2 Participants . . . . . . . . . . . . . . 139

8.2.3 Experimental procedure . . . . . . . . . . . . . 139

8.2.4 Game structure . . . . . . . . . . . . . . . . 139

8.2.5 Resources and scoring . . . . . . . . . . . . . . . 140

8.2 .6 Alien language . . . . . . . . . . . . . . . . . . . . . . 140

8.2.7 Comparison of experiments . . . . . . . . . . . . 140

8.3 Results . . . . . . . . . . . . . . . . . . . . . 140

8.3.1 Giving behaviour . . . . . . . . . . . . . . . . 141

8.3.2 Recognising team-mates . . . . . . . . . . . . . 145

8.3.2.1 Feedback questionnaires . . . . . . . . . . . 145

8.3.2.2 Explicit and implicit judgements in the Competitive condition . . . . . . . . . . . . . 148

8.3.3 Divergence. . . . . . . . . . . . . . . . . . . . . . . 149

8.3.4 Comparison with score . . . . . . . . . . . . 150

8.4 Strategy . . . . . . . . . . . . . . . . . 150

8.4.1 Identification strategies . . . . . . . . . . . . . 150

8.4 .2 Summary of results . . . . . . . . . . . . . . . . 151

8.5 Discussion . . . . . . . . . . . . . . . . . . . . . 152

Chapter 9 Discussion of experimental results 153

9.1 Summary of findings . . . . . . . . . . . . . . . . . . . 153

9.2 Bridging the alien-human gap . . . . . . . . . . . . . . . . 155

9.2.1 Serbo-Croatian disintegration . . . . . . . . . . 156

9.2.2 Classic sociolinguistic studies . . . . . . . . . . 157

9.2 .3 Midlan High . . . . . . . . . . . . . . . . . . . . . . . . . . . 159

9.3 The role of players' strategies _. . . . . . . . . . . . . . . . 160

9.4 The role of accommodation/interactive alignment . . . . . . . . . . 164

9.5 Social selection . . . . . . . . . . . . . . . . . . . . . . . . 169 
9.6 Conclusion . . . . . . . . . . . . . . . . . . . . 171

Chapter 10 An evolutionary perspective 173

10.1 A bias towards language . . . . . . . . . . . . . . . 173

10.2 Selection for variability . . . . . . . . . . . . . . . 178

10.3 Bilingualism and maturational constraints . . . . . . . . . . . . . 182

10.4 A tentative sketch . . . . . . . . . . . . . . . . . 186

$\begin{array}{lll}\text { Chapter } 11 & \text { Conclusions } & 191\end{array}$

11.1 Summary of experimental findings . . . . . . . . . . . . . . . 191

11.1.1 First experiment . . . . . . . . . . . . . . . 191

11.1.2 Second experiment . . . . . . . . . . . . . . . . 192

11.1.3 Third experiment . . . . . . . . . . . . . . . . . 193

11.1.4 Fourth experiment ................ 193

11.2 How the divergence came about . . . . . . . . . . . . . . . . 194

11.3 Future developments . . . . . . . . . . . . . . . . . 195

$\begin{array}{ll}\text { Appendix A Experiment materials } & 199\end{array}$

A.1 Instructions for the experiments . . . . . . . . . . . . 200

A.1.1 First experiment . . . . . . . . . . . . . 200

A.1.2 Second experiment: Competitive condition . . . . . . . 206

A.1.3 Second experiment: Cooperative condition . . . . . . . . . . 210

A.1.4 Third and fourth experiments: Competitive condition . . . . 217

A.1.5 Third and fourth experiments: Cooperative condition . . . . 221

A.2 Feedback questionnaires . . . . . . . . . . . . . . . . 224

A.2.1 First experiment . . . . . . . . . . . . . . . . . 224

A.2.2 Second experiment . . . . . . . . . . . . . 225

A.2.3 Third and fourth experiments . . . . . . . . . . 227

$\begin{array}{ll}\text { Appendix B Alien language cartoons } & 229\end{array}$

$\begin{array}{ll}\text { Appendix C Calculating divergence } & 237\end{array}$

$\begin{array}{ll}\text { Appendix D Published papers } & 241\end{array}$ 
References 



\section{List of Tables}

2.1 Summary of social behaviour types . . . . . . . . . . . . . . . . 9

5.1 Summary of results from first experiment. . . . . . . . . . . . 87

5.2 Example variable set from first experiment . . . . . . . . . . . . . . 92

5.3 Mean distances between players in example set . . . . . . . . . . . . 92

6.1 Summary of differences between first and second experiments . . . . 108

6.2 Summary of results from second experiment (Competitive condition). 110

6.3 Summary of results from second experiment (Cooperative condition). 111

7.1 Summary of differences between first, second, and third experiments 124

7.2 Summary of results from third experiment (Competitive condition). 125

7.3 Summary of results from third experiment (Cooperative condition). 126

8.1 Summary of differences between all four experiments . . . . . . . . 140

8.2 Summary of results from fourth experiment (Competitive condition). 141

8.3 Summary of results from fourth experiment (Cooperative condition). 142

8.4 Summary of mean gifts . . . . . . . . . . . . . . . . . . . . 142

9.1 Summary of results for conditions . . . . . . . . . . . . . . . . . 154

9.2 Summary of accommodation rate (Competitive conditions) . . . . . 168

9.3 Summary of accommodation rate (Cooperative conditions) . . . . . 168

10.1 Four kinds of human vocalisation . . . . . . . . . . . . . 187

C.1 Example variable set from first experiment . . . . . . . . . . . . . 237

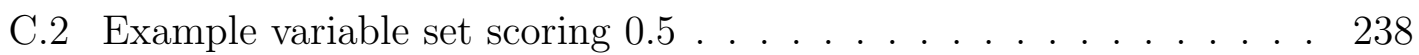

C.3 Mean distances from example set . . . . . . . . . . . . . . . 238

C.4 P-values for divergence depending on shuffling method (Competitive

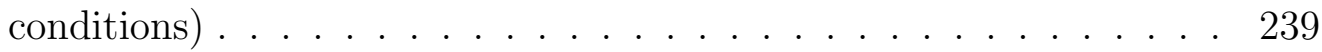

C.5 P-values for divergence depending on shuffling method (Cooperative conditions) . . . . . . . . . . . . . . . . . . . . 239 



\section{List of Figures}

5.1 Diagram of experiment setup _ . . . . . . . . . . . . . . . . 79

5.2 Diagram of game structure . . . . . . . . . . . . . . . 80

5.3 Examples of CHAT and EXCHANGE stages . . . . . . . . . . . . 82

5.4 Examples of FEEDBACK stage and final screen . . . . . . . . . . . 82

5.5 Frequency of sentence types . . . . . . . . . . . . . . . . . . . . 94

6.1 Gifts per round in Game 3 of the Competitive condition . . . . . . 119

7.1 Scatterplot of recognition rate against score . . . . . . . . . . . . . 131

7.2 Diagram of experimental design . . . . . . . . . . . . . . . . 135

8.1 Gifts per round in Game 1 of the Cooperative condition . . . . . . . 146

8.2 Gifts per round in Game 2 of the Cooperative condition . . . . . . . 147

B.1 Index of cartoons . . . . . . . . . . . . . . . . . . . . . . . . . 229

B.2 Cartoon: "hello!" . . . . . . . . . . . . . . . . . . . . 230

B.3 Cartoon: "I have fish" . . . . . . . . . . . . . . . . . . . 231

B.4 Cartoon: "do you want fruit?" "Yes!" . . . . . . . . . . . . . . . 232

B.5 Cartoon: "have much corn" . . . . . . . . . . . . . . . . . . . 233

B.6 Cartoon: "no need meat!" . . . . . . . . . . . . . . . . . . . . . . . 234

B.7 Cartoon: "I have no water" . . . . . . . . . . . . . . . . . . 235

B.8 Cartoon: "goodbye and thanks!" . . . . . . . . . . . . . 236 



\section{CHAPTER 1}

\section{Introduction}

Two facts about language are undeniable. The first is familiar to most people from everyday experience: it is that language communicates not only propositions, but also social information about the speaker. The second fact requires some knowledge of history, though it is enough to be dimly aware that French, Italian, and Spanish all used to be called Latin. What can be inferred from this is that languages change, and not only that - that one language can diverge into multiple accents and dialects and, ultimately, new languages. Such data provoke a hypothesis: perhaps the two facts are related. Indeed, they must be. If there were no such things as accents and dialects, then language could not communicate social information in the way that it does. Perhaps, though, the causality also runs in the opposite direction: does the need for social marking lead languages to diverge into new dialects (or at least hasten their divergence)? This is the question this thesis addresses.

\subsection{Why suicide bombers shouldn't have Yorkshire accents}

Whenever we speak, we give away all sorts of social information about ourselves: where we are from; what kind of school we went to (or go to); what kind of job we do; even what we like to do in our free time (see Section 9.2.3). We tend to forget, since we take it for granted, that this is somewhat curious. There are in fact two parts to the question of why it is the case: the first is the question of why speech 
varies according to such factors; the second is why listeners are so good at learning to interpret the variation - what is the use of doing so?

It is this second question that looks most in need of an answer. The fact that speech patterns vary at all looks as if it can be explained in terms of random drift, and the fact that people from the same community sound like each other may be explicable in terms of variation in the frequency with which people talk to each other (although see Section 1.2 below). But this does not explain why we pay so much attention to the variation. Not only do we notice it, but we attach importance to it. Enough importance, in fact, that parents pay large sums of money to send their children to schools where they will learn not to talk like them. Enough importance that people take time out of their day to write to the $\mathrm{BBC}$ to complain about Americanisms, or about Radio 4 announcers sounding Jamaican (Kirby: 1 April 2006). Enough importance that, after the $7 / 7$ London suicide bombings, when a video of one of the bombers was shown on Al-Jazeera, the Times reported:

Shehzad Tanweer, 22, a university dropout from Beeston, Leeds, is shown speaking with a Yorkshire accent, wearing a Muslim headscarf and jabbing his finger at the camera. (Fresco et al.: July 6 2006)

It is fascinating that a respected British national newspaper should find it worthy of mention, in the first paragraphs of an article, that a young man from near Leeds spoke with a Yorkshire accent. There may even be a clue here as to why accents matter to us; and there may be a further clue in the limits that exist on our ability to distinguish them. We are rather good at telling if another person is not from the same place, or the same social group, as we are, but we tend not to be very good at distinguishing between all the different "not-one-of-us" accents, except where this happens to be especially relevant to us. A native of Edinburgh, for instance, is likely to be rather good at identifying Glaswegians by the way they speak (and distinguishing them from people from Stirling, or Perth); and, in principle, this should not be a very hard task, as there are relatively significant differences to pick up on. In spite of this, however, visitors from outside Scotland tend to find it rather hard to make the distinction. It is not, therefore, that human beings are extremely well attuned to all linguistic variation; it is rather that we are very good at learning the (often subtle) distinctions that are relevant to us. This suggests that 
the ability is, primarily, about identifying outsiders to one's own group and, perhaps secondarily, about distinguishing between outsiders we encounter frequently. The very point of the ability, it would seem, is that it allows us to tell if someone is part of our group or not. This is why it shocked people that a Muslim terrorist had a Yorkshire accent instead of a Middle-Eastern one; and this is why some British people worry about Americanisms on Radio 4: because they feel Radio 4 belongs to them, and putting on the speech patterns of another group feels like disloyalty.

We are still in the realm of proximate explanations, however - why, after all, do we care about whether or not someone is part of our group? The best ultimate explanation for such behaviour has to do with cooperation, and the mechanisms that support it, which depend on an ability to distinguish between individuals who have a local reputation to consider and outsiders who do not. This will be discussed in detail in Chapter 2.

\subsection{The drive to sound different}

If this is the explanation for why we pay such attention, and attach such value, to linguistic variation, does it also play a role in explaining how the socially structured variation comes about in the first place? It was suggested above that socially structured variation might be explicable simply in terms of random drift and variation in the frequency with which speakers interact, given that people come to speak most like the people they talk to the most. This is the the view of Trudgill (2004, 2008a), and, as the most parsimonious explanation, it should be our null hypothesis. On the other hand, Atkinson et al. (2008: 588) found evidence for "rapid bursts of change associated with the emergence of new languages." This suggests that there may be something more going on, at least some of the time: that, as was suggested in the first paragraph of this introduction, the need for social marking may drive people to sound different - or rather, to sound less like some people and more like others.

It should not be understood from this that people set about trying to create new dialects. Atkinson et al. (2008) begin their article with Noah Webster's assertion that: 
as an independent nation, our honor requires us to have a system of our own, in language as well as in government. (Webster 1789: 20)

This is misleading. As will be discussed more in Section 9.2.1, it seems highly unlikely that this kind of explicit planning is how new dialects usually come about, or even that this is how American English came about (certain spelling conventions excepted). If identity plays a role in language divergence, then it is rather more likely to play a role in the everyday interactions of individuals and the choices they make, whether they are aware of it or not, in how to use language. It is here, in these small-scale strategies, that "social selection" can be said to play a role: the (conscious or unconscious) selection of linguistic forms for social reasons. The opposition between this social-selection model and the interaction-frequency model in explaining language divergence will be discussed in detail in Chapter 3, and is the focus of the central question of this thesis.

\subsection{An underused methodology}

The question will be addressed by means of experimental simulation. This is a methodology that has a relatively long history, and which has been employed with some success more recently to investigate the origins and evolution of language (Galantucci 2005; Kirby et al. 2008; Scott-Phillips et al. 2009). It has not been much used to research change in modern language, however. There are likely to be two main reasons for this. First, evolutionary linguists have no real-life data to work with that can shed a direct light on language origins; they are forced to take more indirect routes and create data through simulation. Historical linguists, by contrast, have centuries of recorded language to work with, eked out by reconstructions of proto-forms from periods before literacy. Given this, simulated data is less necessary, although computational and mathematical simulations have begun to show their worth (Nettle and Dunbar 1997; Nettle 1999; Livingstone and Fyfe 1999; Livingstone 2002; Oudeyer 2005; de Oliveira et al. 2008; Baxter et al. 2009; Kirby to appear).

Chapters 5 to 9 will present a series of such experiments designed to address the central question of this thesis: the role of social factors in language divergence. 
This paradigm involved having participants learn an "alien language" of twenty made-up words and use it to play a game in which they negotiated with each other, anonymously, to exchange resources. In one condition, players were divided into teams, and the game was set up such that it was advantageous to give away resources to teammates, but not to opponents. Frequency of interaction was also manipulated. This allowed two main questions to be investigated: first, were players able to come up with sociolinguistic markers in a made-up language they had only just learnt, and without the means to discuss their strategies explicitly; and, if so, under what conditions? Second, did this behaviour lead the alien language to diverge significantly? The strategies that players used, their patterns of gift giving, and the relationship between their score in the game and other factors were all also investigated.

\subsection{Other questions}

Questions have a habit of inviting their friends. The use of the word "selection" in the title of this thesis raises questions about cultural evolution: do the commonalities between language change and biological evolution, new-dialect formation and speciation, imply they are fundamentally examples of the same process? This will be addressed in Chapter 4. Questions more directly concerned with biological evolution will be discussed in Chapter 10, along with some suggestions of how they might be answered: are human beings innately biased to pay special attention to linguistic cues (as opposed to other cultural and biological cues, such as race)? Has a pressure for social marking led to selection for variability in language? How should we explain children's impressive ability to learn several languages at once? This chapter will finish with a tentative sketch of the evolutionary path envisaged. After this murky peer at our distant past, Chapter 11 will look forward to future research. 



\section{Chapter 2}

\section{The problem of cooperation}

Cooperation is enormously important. It is easy to suppose, and it frequently is supposed, that cooperating is something human beings do, but which is otherwise rare. This reflects a serious misunderstanding: cooperation is everywhere. It has played a fundamental part in the history of life, and at all levels of life (Dawkins 1989: 37-45; Axelrod and Hamilton 1984). Maynard Smith and Szathmáry (1995: 4) note that the most important feature common to their major transitions in evolution "is that entities that were capable of independent replication before the transition can replicate only as part of a larger whole after it." Not only our societies, but also our bodies, our cells, and the very DNA that codes for the proteins of which our cells are made are testament to the power of cooperation. Yet explaining cooperation in evolutionary terms is problematic. As Maynard Smith and Szathmáry (1995: 4) put it: "why does not selection between entities at the lower level disrupt integration at the higher one?"

\subsection{Defining cooperation}

Before going further, it would be useful to be clear what is meant by cooperation, and related terms like "altruism". Such terms are not always used as clearly and as consistently as they might - particularly outside biology — and this has led to confusion. West et al. (2007) provide a good summary of the confusion and clarify key terms, in general following established practice in biology, although also making recommendations of their own, and this thesis will follow them in their use of terms. 
Along with Hamilton (1964) in his classification of social behaviours, they define cooperation as follows:

a behaviour which provides a benefit to another individual (recipient), and is selected for because of its beneficial effect on the recipient. (416)

Several points about this definition should be borne in mind:

- Benefit is to be understood as an absolute, not a relative, fitness benefit. The important question is whether recipients produce more offspring than they would in the absence of the cooperative behaviour. Even if some individual recipient benefits relatively more than other recipients from the behaviour, in other words, it still counts as cooperative for all beneficiaries.

- The fitness benefits to the recipient must be direct. A fitness benefit is direct when it increases the number of offspring that a given organism produces, and which survive into adulthood; it is indirect when it increases the number of surviving offspring produced by individuals carrying the same cooperative allele. If behaviour were called cooperative on the basis of only an indirect benefit to the recipient, then selfish behaviour would be included, since it indirectly benefits close relatives of the actor (stealing from one's siblings would be cooperative, because the effect on one's own reproductive success would indirectly benefit the same siblings!). Indirect fitness is important to accounts of cooperation, but chiefly in explanations of apparently altruistic behaviour (see Section 2.2.1), where the indirect benefit to the actor - not the recipient-plays a key role.

- The fitness benefit in question is to be understood not as the short-term benefit of a particular cooperative act, but in terms of average lifetime payoffs; behaviour may thus be cooperative even if, on a particular occasion, it happens to "misfire". For example, feeding your children is a cooperative act, even if, on one occasion, they are unlucky enough to get food poisoning.

- The costliness of a behaviour should be understood in the same terms: behaviour is costly or harmful to the extent that the average lifetime payoff from it is negative. 
- Cooperative behaviour should be selected for. Parasites benefit from various behaviours of their hosts, but this does not mean that these behaviours are cooperative.

Finally, it will be noticed that no reference is made to the actor's payoff under this definition. Altruistic behaviour, which can be defined as behaviour costly to the actor and beneficial to the recipient, is, therefore, a subset of cooperative behaviour. West et al. (2007: 418) suggest "mutual benefit" as a term to describe cooperative behaviour that is directly beneficial to both parties. ${ }^{1}$

Non-cooperative social behaviour can be distinguished along the same lines: selfish behaviour is behaviour beneficial to the actor and costly to recipients (i.e. has an average negative fitness payoff for other individuals); behaviour that is costly to both the actor and the recipient is termed "spiteful" (ibid.). This is summarised in Table 2.1, in which "+" represents a direct benefit, and "-" represents a direct cost. $^{2}$

\begin{tabular}{lcc}
\hline & Effect on recipient \\
& + & - \\
\hline Effect on actor & & \\
+ & Mutual benefit & Selfishness \\
- & Altruism & Spite \\
\hline
\end{tabular}

Table 2.1: Summary of social behaviour types (cf. Hamilton 1964; West et al. 200\%: 418)

\footnotetext{
${ }^{1}$ This should be distinguished from "mutualism", which — while it is often used in this senseis also frequently used to refer to interspecific cooperation. See West et al. (2007: 418) for a discussion. Clutton-Brook (2009), by contrast, uses it in to refer to instances where actors benefit by being a part of a benefiting group.

${ }^{2}$ If the minus signs are taken to represent an indirect benefit instead of a direct cost, then the left half of the table remains the same, since it is indirect benefit that explains altruism. For both cells in the right half of the table, however, the meaning of "recipient" changes: this indirect recipient must now be a relative of the actor. The top right cell would thus still represent selfish behaviour, but the bottom right would now, like the bottom left, represent altruism, the difference being that there is some other, direct, recipient - a relative of both — not included in the table.
} 


\subsection{Explaining cooperation}

Explaining cooperative behaviour has long been an intriguing problem for evolutionary thinkers, including Darwin (1879/2004: 119-72). It should not be assumed, however, that it poses a problem for evolutionary theory in particular. Social contract theory, in which the seventeenth- and eighteenth-century philosophers Thomas Hobbes, John Locke, and Jean-Jacques Rousseau stand out as the best known figures, is an early-modern response to very much the same question, which has engaged philosophers in all eras. It is this:

Under what conditions will cooperation emerge in a world of egoists without central authority? ... in situations where each individual has an incentive to be selfish, how can cooperation ever develop? (Axelrod 1984/1990: 3)

Nor is the problem a mainly hypothetical one, or one that concerns only human behaviour (as noted at the start of this chapter, cooperation is to be found throughout nature). In spite of Axelrod's vivid choice of words, the problem does not lie in an irreconcilable mismatch between an evolutionary world of selfishness and some creationist world of altruism. It is clear that cooperation can arise from selfishness; the problem of explaining cooperative behaviour in evolutionary terms is in pinning down precisely how it contributes to the actor's fitness. A first distinction to be made is between direct and indirect benefits ${ }^{3}$ (see Section 2.1, page 8, for a definition of these terms, and Section 2.2.1 for more discussion), although, of course, both kinds of benefit may be involved in a given case. If it is direct (i.e. if the behaviour is mutually beneficial), then either a group of actors draw a shared benefit from some cooperative enterprise, or an actor is compensated for a particular cooperative act by cooperative acts from other individuals. The problem becomes one of explaining how such a system is enforced. If the benefit is indirect (and the behaviour is thus altruistic), then the problem lies in uncovering the level of kinship necessary for such behaviour to be adaptive, and the means by which the actor establishes this.

\footnotetext{
${ }^{3}$ Actors and recipients differ in this respect. It will be recalled that cooperative behaviour is defined as behaviour that directly benefits some recipient. The benefit to the actor need not be direct.
} 
Section 2.2.1 will discuss indirect fitness benefits and kin recognition in more detail, while Section 2.2.2 will discuss enforcement in mutually beneficial behaviour.

\subsubsection{Indirect benefits and altruistic behaviour}

Altruism is cooperative behaviour in which there is no direct benefit to the actor. In view of this, it should be noted that some behaviour that appears altruistic is in fact mutually beneficial. The term "altruism" has even been applied in the literature to such behaviour. For example, behaviour has been termed "weakly altruistic" where it is costly to the actor relative to the individuals it interacts with in its group (see Wilson 1975, 1977; cf. the use in Boyd et al. 2003 of the term "altruistic punishment", which may or may not be strictly altruistic). As West et al. (2007: 420-1) show, however, such behaviour can in fact increase the individuals' direct fitness. Similarly, the term "reciprocal altruism" (introduced by Trivers 1971) is often used for situations where a cooperative act is rewarded by another cooperative act that benefits the first actor. This reward, however, means that the behaviour is not in fact altruistic in a useful sense of the term, and is instead mutually beneficial. Several writers (e.g. Hamilton 1996; West et al. 2007) prefer alternative terms like "reciprocity" for this reason.

True altruistic behaviour that does not involve an indirect fitness benefit for the actor (i.e. does not on average provide a direct fitness benefit to other bearers of the gene) should not be expected to evolve by natural selection. This is as much as saying that for behaviour to evolve by natural selection, it must be selected for. A classic example of altruistic behaviour, clearly explicable by indirect fitness benefits, is of a parent aiding its young. This is simply a particularly salient case of kin selection; any altruistic behaviour can be explained in this way provided the average indirect benefit outweighs the cost of the behaviour. Formally, we should expect genes for any behaviour to increase in frequency whenever $r B>C$, where $r$ is the genetic relationship between the actor and recipient, $B$ is the benefit to the recipient of the behaviour, and $C$ is the cost to the actor. This is known as Hamilton's Rule (Hamilton 1964). 
It is important to note that this does not mean every altruistic act must be beneficial to the actor; such behaviour can be expected to operate on a rule-ofthumb basis (cf. Hutchinson and Gigerenzer 2005), provided that the average net payoff is positive. This allows a certain amount of abuse, as can be seen in the behaviour of cuckoos, who lay their eggs in the nests of other bird species. It is not the case that the mother birds targeted by cuckoos have no mechanisms for identifying their own young; they do not, for example, try to feed rocks. Rather, it is these very identification mechanisms that cuckoos exploit - in this case, the genetic instruction to treat as kin "any small bird sitting in the nest that you built" (Dawkins 1989: 102; cf. Komdeur et al. 2008: 181; see Komdeur and Hatchwell 1999 for a review of kin-recognition mechanisms in birds).

\subsubsection{Mechanisms of kin recognition}

There are several possible kinds of kin-recognition mechanism (see Hepper 1991, 2008 for a review). ${ }^{4}$ One solution might be provided by a "green beard", where a gene, or group of linked genes, produces three things: a recognisable phenotypic effect; recognition of this trait in others; and preferential behaviour towards individuals exhibiting the trait. The idea was first put forward by Hamilton (1964), and given a name by Dawkins (1989: 89). Though theoretically possible, such effects have generally been assumed to be rather unlikely to arise. As Dawkins puts it:

Green beardness is just as likely to be linked to a tendency to develop ingrowing toenails or any other trait, and a fondness for green beards is just as likely to go with an inability to smell freesias. It is not very probable that one and the same gene would produce both the right label and the right sort of altruism. (ibid.)

In spite of this, Keller and Ross (1998) provide evidence for one - now frequently cited - case in ants, and there are other cases to be found in microbes, where the link between genotype and phenotype is relatively simple (West and Gardner 2010).

\footnotetext{
${ }^{4}$ Kin-recognition aids the evolution of altruism by helping propagate the genes that underlie it. It might be objected that "trait-recognition" could do the job just as well, if not better, since altruistic behaviour may be more unambiguously identifiable than kinship. This is misleading, for if the altruistic behaviour identified in such a case were based on different genes, then there would be no indirect benefit to the actor, and there would still be pressure for kin-recognition. If there were instead a direct benefit to the actor in rewarding such behaviour, then this would be a case of mutual benefit, not altruism.
} 
More broadly, however, various more general heuristics can be expected to be employed for recognising kin (Dawkins 1989: 99-107), including physical resemblance and childhood relationships. Shepher (1971) found, for example, no cases of marriage or heterosexual activity between individuals raised in the same peer group on a kibbutz, the implication being that the instinct for incest avoidance was operating with a kin-recognition heuristic based on upbringing patterns. There is also evidence that grandparents make quite subtle adjustments in behaviour on the basis of the likelihood of kinship; Laham et al. (2005) found, for example, that grandchildren tend to have closer relationships with maternal grandparents than with paternal ones, while Michalski and Shackelford (2005) found that this had an effect on grandparents' actual investment of time and money. Other behaviour may serve to reinforce assumptions of kinship; Daly and Wilson (1982) found that newborn babies are alleged to resemble their fathers far more often than their mothers.

On the other hand, kin-recognition mechanisms may work well enough on a much more general level: in populations with sufficiently low levels of migration, or limited dispersal (such groups are called "low-viscosity" groups), levels of kinship between all members are likely to be sufficiently high for altruistic behaviour to be directed indiscriminately towards other members of the group (Dawkins 1989: 100), although there is simulation evidence to suggest that the increased competition between kin can cancel this effect out (Wilson et al. 1992; Taylor 1992a,b). It is also worth noting that, as behaviour towards adoptees, pets, or other organisms in loco infantis shows, human beings can easily develop feelings of kinship towards unrelated individuals, even when the lack of kinship is obvious to all involved. Explanations for non-kin adoption have made reference to the heuristic nature of kin-recognition (Dawkins 1989: 101), evolutionary lag (where adoption is no longer adaptive for the adopters, but once was, owing to the low-viscosity of the group; see Barash 1982: 327), or mutual benefit (where the adopted child provides some direct benefit to the adopters; see Lindgren and Pegalis 1989). We should of course be careful of simply assuming that relationships between adopted children and their parents are necessarily exactly like those between parents and genetic offspring, whether for better or worse. It is at least the case that some adoptive parents lack a sense of entitlement to their children, for which they may compensate; however, Miall 
(1987) explains this with reference not to the parents' own views, but to societal stigma aimed primarily at infertile couples and couples who choose to be childless. She found that attitudes towards adoption are influenced positively by familiarity with families that have adopted children.

In all the enforcement mechanisms described above, there remains a potential to cheat the system: outsiders can become attached to low-viscosity groups, pose as kin, or even pose as offspring. Even a green beard is not foolproof - if a phenotypic trait can evolve that is genetically linked to cooperative behaviour, then it can presumably evolve without that link too. Of course, simply not being kin need not matter; a population of individuals behaving altruistically to one another is barely distinguishable from a population of indirectly reciprocal cooperators (that is, individuals who cooperate with others on the basis that the others are also generally inclined to cooperate), and if the kin-cheat returns favours, then it causes relatively little harm to the cooperative system. But the cheat who poses as kin and also gives little in return for favours can also do very well indeed. In this case some form of enforcement may become necessary, of the kind described in the next section.

\subsubsection{Enforcement in mutually beneficial behaviour}

Mutually beneficial behaviour is cooperative behaviour where both the actor and the recipient benefit directly. As benefit is to be understood as a lifetime fitness benefit (see Section 2.1), the benefit to the recipient may consist in being the recipient of such behaviour at a later date. Alternatively, two or more individuals might work together to achieve some goal from which they all benefit, or one individual might exchange skills or goods with another. All this counts as mutually beneficial behaviour, and the three examples just given are hardly exhaustive. In some cases, enforcement is integral to the activity: to have a favour returned is to be rewarded for doing the favour. Nor does the relationship between one favour and another need to be explicit - an individual is rewarded for a propensity for doing favours by living in a population of similarly inclined individuals. There are other kinds of enforcement, however, which may be more negative, and several-often overlapping - terms are used in the literature. They fall into two broad categories: 
- Positive enforcement. Several terms are used:

- Reward: Cooperative behaviour is directly rewarded, either by the recipient of the behaviour or a third party.

- Reciprocity: This, first described in evolutionary terms by Trivers (1971) as "reciprocal altruism", can be summed up as "I'll scratch your back if you scratch mine". It involves a direct reward, as the individuals involved reward each other for cooperative behaviour. Where an individual behaves cooperatively on the assumption that the behaviour will be reciprocated at a later date, Gintis (2000) calls this "weak reciprocity" (cf. "Strong reciprocity", described below).

- Indirect (reputation based) reciprocity: An individual is made the recipient of cooperative behaviour on the basis of their reputation as a cooperative individual - meaning that members of a group are constantly reassessed (Alexander 1987; for formal models of this see: Nowak and Sigmund 1998a,b; Panchanathan and Boyd 2003).

- Strong reciprocity: This term was introduced by Gintis (2000) to describe individuals who are "predisposed to cooperate with others and punish non-cooperators, even when this behavior cannot be justified in terms of self-interest, extended kinship or reciprocal altruism" (169). In other words, there is a direct fitness benefit, but not one that relies on the cooperative behaviour being directly rewarded, or on the expectation of direct reciprocation. A strong reciprocator benefits by being a member of a group that is made more successful by the behaviour in question. The explanation, in spite of Gintis's claim, thus relies ultimately on selfinterest (cf. West et al. 2007: 426, who argue that such claims confuse proximate and ultimate explanation).

- Negative enforcement. Again, several terms are used:

- Policing: Behaviour in a group is monitored and uncooperative behaviour is punished (see below). 
- Punishment/sanction: ${ }^{5}$ Uncooperative behaviour (such as failing to return a favour) is directly punished, either by a directly involved individual, or by some third party. Punishment involves incurring some sort of cost to the malefactor, and may consist solely in spreading word that the individual is uncooperative. Such reputation-based punishment requires a sophisticated communication system, but, once such a system is in place, it is comparatively cheap (Dunbar 1996).

The role of culture in all these kinds of enforcement may, of course, be significant (Richerson et al. 2003; Henrich et al. 2010). It is not the case, of course, that such mechanisms are foolproof. They can all be circumvented. The next section discusses this.

\subsection{The problem of freeriders}

The terms "free(-)rider", "free(-)loader", and "cheat(er)" are all used more or less interchangeably in the literature to refer to individuals who exploit cooperative systems so that they can enjoy the benefits undeservedly (Boehm 1997, for example, uses all three with no obvious distinction between them). The most commonly used of the three terms is "freerider" and so will be the one employed here, particularly as the compound term "freerider problem" is well established.

In the case of altruistic behaviour, as discussed in Section 2.2.1, a freerider is an individual who poses as more closely related to an altruistic actor than is the case. In the case of mutually beneficial behaviour, a freerider is one who fails to return favours, defaults on deals, contributes less to but enjoys more of the fruits of teamwork, and so on. Such behaviour can be punished, as described in Section 2.2.2. However, such punishment can be avoided by cheats who do not stay around long enough to receive it. Reputation-based punishment (a relatively cheap form of punishment, at least for humans) is useless against an individual who moves faster than the speed of gossip. Therefore, a mobile individual who travels from community to community, exploiting each in turn, can be particularly dangerous

\footnotetext{
${ }^{5}$ These terms both occur in the literature, but there is no obvious attempt to distinguish them (cf. Fehr and Rockenbach 2003; Fehr and Fischbacher 2004; Houser et al. 2008)
} 
(Enquist and Leimar 1993). Such individuals can also exploit altruistic behaviour in groups with limited dispersal (see Section 2.2.1).

Learning, through reputation and observation, to avoid cooperating with specific individuals is a useful strategy, but this will do nothing to protect against exploitation by individuals you happen not to have met or heard of yet (and, even if you have been warned of a particular cheat, this does not mean you will recognise them when you meet them). In the face of a serious threat from outsiders, two approaches remain: decreasing one's investment in cooperation and compensating by increasing the number of interactions, or limiting the recipients of one's cooperative behaviour to individuals who are likely to form part of the same cooperative network - that is, treating newcomers with suspicion. This may involve refusing to cooperate with any newcomer, which is suitable for extreme situations, but which seriously limits the potential for mutually beneficial interaction. Not all newcomers, after all, are equally likely to defect. Newcomers from nearby are more vulnerable to gossip, for example; an individual from the next village has more of a local reputation to maintain than someone from another country. Among neighbouring communities there will also be distinctions: members of hostile communities are more likely to defect than members of friendly ones. This means that mechanisms which allow information to be inferred about an unfamiliar individual's origins are valuable and aid cooperation. Inferring such information is not trivial; that it might seem so chiefly reflects the numerous mechanisms we have evolved (or invented) for ingroup recognition.

\subsection{Identifying oneself and others}

Information about an individual's group membership can be stored in a variety of places. Section 2.2.1 gave some examples of heuristics that might be relied on to identify kin, mainly based not on identifying features of the individual in question, but on the "normally ... reliable correlation between genetic relatedness and the spatial/temporal component of association" (Halpin 1991: 222; see also Komdeur et al. 2008). Such heuristics are reliable in relatively low-viscosity groups, but can obviously become very unreliable if non-kin are regularly present. In these cases, it may become necessary to rely on associating specific cues with individuals. 
Beecher (1982) coined the term "signature system" for cues that exhibit greater inter-individual than intra-individual variation, and are thus suitable for identifying individuals.

Above the level of the individual, such labels can become associated with groupswhat Halpin (1991) calls "group cues". He notes that these tend to be either individually-distinctive cues of the sort mentioned above, or a combination of such cues. They also tend to be environmentally acquired rather than genetically determined (223). That last fact means that such cues can be used for groups not defined by genetic relatedness. Cues of this sort need not necessarily even be exhibited on the organism in question, as demonstrated by animals that scent-mark. To take a very specifically human example: governments (and other institutions) often store quite extensive information about people, and issue passports for the explicit purpose of identifying people's membership of particular communities. Such documents, of course, may or may not be carried by the person referred to in them, but technology — such as photography and fingerprinting - has long been employed to strengthen the links between the document and the individual referred to in it.

A government database can be seen as an externalised version of what human beings, and other animals, possess without the intervention of technology: (mentally) stored information about other individuals. Such information can, when transmitted through communication systems, behave somewhat like other affiliation cues stored in the environment, although access to them (as in the case of government databases) is restricted by the possessors of the information. The sort of information stored mentally might range from a list of individuals in one's own social group to detailed information about the social relationships between these individuals (and oneself) and individuals in other groups, as well as the relationship between social groups themselves. It has been argued convincingly that the relatively large size of anthropoid primate brains is best explained by the cognitive demands of the relatively complex social structures of these primates, based on networks of non-reproductive pairbonds (Byrne and Whiten 1988; Dunbar 1998; Dunbar and Shultz 2007; cf. Herrmann et al. 2007). There is, moreover, good evidence of the importance to hunter gatherers of both regional and long-range social ties, which 
Whallon (2006: 261) refers to as a "'safety net' ... in times of local resource scarcity or failure".

The amount of information that can be stored mentally is clearly limited by the memory capacity of the individual, but can be supplemented and extended (at least in human beings) by such external devices as address books and social-networking websites, at which point the distinction between environment and individual is again blurred (cf. Clark and Chalmers 1998). Stored information, however, will always be somewhat wanting: first, storage capacities put a limit not only on how much information can be stored, but also on speed of retrieval, even when cognition is supplemented by technology. Second, such information as can be stored must be gathered from the environment in the first place. Third, information previously gathered from the environment is inherently more likely to be out of date than information currently in the environment. Fourth, stored information is of much less use with new acquaintances. Therefore, while it is reasonable for extensive and detailed information (gathered over long time periods and frequently updated) about an inner-circle of contacts to be stored in memory, this needs to be supplemented by heuristics and mechanisms for interpreting cues directly from the environment or the individual in question.

\subsubsection{Markers}

This brings us to the case where individuals carry information about their affiliations on their person. To the extent that these are on display, they can be termed markers. ${ }^{6}$ Such social markers can be useful both to their bearers and to others.

To its bearer, a marker is useful if it signals membership or non-membership of groups in such a way as to maximise the fitness benefit for that user. In many cases this may involve appearing to belong to the same group as other individuals in an

\footnotetext{
${ }^{6}$ This term is chosen for its relative transparency, and because it is already used in this sense in the literature (e.g. Boyd and Richerson 1987; Nettle and Dunbar 1997; McElreath et al. 2003). However, it should be noted that a distinction is normally drawn in sociolinguistics between markers, stereotypes and indicators. With regard to linguistic variation, indicators are below the level of conscious awareness, but correlate with social (though not stylistic) factors; stereotypes correlate with social factors, but are above the level of conscious awareness, and attract positive or negative evaluation; markers may or may not be above the level of conscious awareness, and correlate with social and stylistic factors, but tend not to attract evaluation. For simplicity's sake, such distinctions will be ignored in this chapter.
} 
interaction, or at least the majority of them; it may be more beneficial, however, to appear to be of the same group as the most influential minority, or at least of a group favourable to them. In other cases it may be equally, or even more, important to signal one's lack of connection to a particular group. It would seem, therefore, that there would be pressure for individuals to develop chameleon-like markers that are able to change to suit circumstances. There are two pressures in the opposite direction, however: first, to be perceived as too fluid in one's affiliations is quite dangerous, as it identifies an individual as considerably less trustworthy (it is possible to be considered too charming). It will almost always be preferable to be considered an honest member of the wrong group than a false member of the right group. Second, there is pressure on observers to attach more weight to more reliable markers; this means that the most trusted markers are likely to be those that are inherently hard to fake, or change.

\subsubsection{Criteria for good markers}

To individuals other than the bearer, then, a social marker is useful to the extent that it provides accurate information about the bearer's identity and affiliations. In order to be accurate, it must fulfil several criteria. Here a distinction must be drawn between variables and variants: a variable is a trait that can vary (e.g. hair colour), and a variant is a particular instance of that variability (e.g. red hair). ${ }^{7}$ The ideal marker is one where a given variant is costly to obtain and to imitate (an imitation being a trait designed, whether by natural selection or cognitive means, to resemble a trait exhibited by another individual). ${ }^{8}$ This recalls theories of honest signalling in biology, such as Zahavi's Handicap Principle (Zahavi 1975, 1977; Zahavi and Zahavi 1997). Costly signalling has also been put forward as an explanation for

\footnotetext{
${ }^{7}$ Boyd and Richerson (1985: 33) contrast cultural variants further with "cultural repertoires", which consist of the information an individual inherits culturally - a particular collection, in other words, of cultural variants.

${ }^{8}$ The term "imitation" can be used in two ways. First, it is a name for a process by which something is copied; second, it is a name for the product of that process. In the second sense in particular, the term tends to imply some lack of authenticity. This is at the root of the implied distinction here between the costliness of obtaining a trait and the costliness of imitating it, which are not necessarily the same thing. In fact, there are two ways in which imitating a trait might differ from obtaining it. Either the imitation resembles the actual trait only at a certain level of detail, but not at other levels (consider, for example, a tattooed freckle); or it has a different level of permanence (hair dye lasts less long than natural hair colour, and is not inherited genetically). The second is in fact a special case of the first.
} 
"strongly reciprocal" cooperative behaviour (Gintis et al. 2001; see Section 2.2.2, page 15, of this thesis) and-most relevant to the current discussion-for certain religious behaviours and rituals as costly markers of affiliation and commitment to a religious group (Irons 2001; Sosis 2003; Ruffle and Sosis 2007).

There are two pressures in the other direction. First, the ideal marker must not be too costly to obtain or possess; the cost should not outweigh the benefits of bearing it. Second, the ideal trait must be obtainable by any genuine member of the relevant group. To act as a social marker, a trait should also be suitably salient - as a variable, it should be suitably easy to observe, and a given variant should be suitably distinct from others - a marker is no good if it can only be seen in restricted circumstances, unless those circumstances coincide with the relevant contexts (in which case, the marker is sufficiently salient for those contexts). Finally, as group dynamics are prone to shift, a variable trait should be flexible enough to accommodate this, at least across generations, but ideally within a single lifetime as well. It will be noticed that there is a tension here: on the one hand, a marker should not be so flexible that its bearer can adjust it too easily and use it to deceive (i.e. its variants must be sufficiently costly to obtain and hard to imitate); on the other hand, if it is too inflexible, it can be made unreliable by changes in group dynamics.

Any trait that varies across human populations may be a candidate for use as a social marker. The variation in such traits may be more or less genetically determined: variation in eye colour, for example, is mainly determined genetically; variation in clothing, on the other hand, is dependent mainly on culture and local environment (climate, availability of materials etc.), although this is not to say that there is no genetic component in shaping individual and cultural tastes. There is much interaction between all these influences, and modern technology even allows cultural manipulation of eye colour. Nevertheless, a continuum can be said to exist between those traits in which variation is chiefly genetic in origin and those traits in which variation is chiefly cultural in origin, and the position of a trait on this continuum influences how well it fulfils the criteria of costliness, imitability, and flexibility across generations. The more that variation in a trait is genetically determined, the more costly the trait will be to obtain (one must be born to the right parents to 
possess it). This kind of trait, however, tends to be very inflexible. Traits in which variation is chiefly cultural tend to be more flexible, at least across generations, but tend to be less costly to obtain and easier to imitate. The flexibility of a marker within an individual's lifetime is influenced by the trait's position on a different continuum: relative permanence. For example, tattoos and many other forms of body modification can be said to have a high degree of permanence (and thus be less flexible), while clothing has considerably less. Neither continuum is especially relevant to the salience of a marker, which depends on a wide range of factors: a large tattoo on one's chest is a very salient marker when one's chest is on display, for example, but not at all if it is covered.

There are, then, very many possible sources of markers, and their suitability varies considerably. If it were the case that all Scottish people had red hair, that no English people had red hair, and that hair dye were hard to come by, then red hair might be a useful marker of Scottishness. Nevertheless, such a situation would make it almost impossible for people without red hair to be considered fully Scottish. Of course, there do exist human communities that actively exclude people on the basis of such genetic traits, but this goes beyond what is necessary for protecting one's community from freeriders, and can make it hard for a group to increase its membership, where group augmentation may be beneficial for all members (cf. Kokko et al. 2001). One might replace red hair in the example with a much more flexible, similarly salient, cultural trait like the wearing of kilts. This could be a good marker, but only if kilts were very hard to make or obtain for non-Scots. To return to the real world: it may indeed be reasonable to assume that a person with red hair and a kilt has some strong connection with Scotland, but if any importance hung from the identification, most observers would be inclined to seek some better marker, and most would be especially interested in the speaker's accent. Among all the possible human traits in which variation could be exploited as a marker of identity, linguistic variation seems an especially good source (cf. Nettle and Dunbar 1997: 98). A person's accent is a salient trait in social situations. It is flexible across generations (infants learn whatever speech patterns occur around them) and even within a single lifetime (Harrington 2006). Yet in spite of this flexibility, learning new speech patterns takes a considerable time, and considerable 
exposure to the target; this appears, moreover, to be generally easier - for reasons that are still debated-for children than for adults (see e.g. Penfield and Roberts 1959; Lenneberg 1967; Newport et al. 2001; Abrahamsson and Hyltenstam 2008). Moreover, duality of patterning ${ }^{9}$ means that language has an enormous potential for semantically meaningless variation, which - for speakers familiar with the code - is not detrimental to communicative function.

\subsubsection{Broader explanations for cultural markers}

This chapter has focused on the development of cultural markers that allow individuals to identify themselves as belonging to a particular social group, and it has explained this in terms of a need to distinguish between insiders and outsiders to one's group as a means of avoiding freeriders. There are, however, other reasons for non-functional cultural variation between groups. In a classic study of vocal learning in birds, Nottebohm (1972) gives population identity (alongside kin recognition) as a proximate explanation for geographically defined songbird dialects. The ultimate explanation is that "Panmixia between neighbouring populations hinders the development of local adaptations" (123). Boyd and Richerson (1987) give a similar explanation for ethnic markers in human beings: that they allow individuals to identify suitable models for social learning, and thus allow more accurate cultural adaptation to variable environments. McElreath et al. (2003) make a similar argument and, indeed, argue that this explanation is preferable to the cooperation-based explanation, which (with reference to Nettle and Dunbar 1997) they do not find to be cogent. Their arguments for the latter point, however, are rather briefly stated, and are far from convincing; indeed, it seems they have misunderstood the position they are opposing. There are two arguments. The first is that:

Altruism can evolve only if some cue allows altruists to interact with each other preferentially so that they receive a disproportionate share of the benefits of altruism. One such cue is kinship ... and another is previous behavior. (122)

\footnotetext{
${ }^{9}$ See Hockett (1960). This refers to the fact that language consists of two layers: a meaningless layer of sound units - phonemes - which is associated, arbitrarily, with a layer of meaningful units - morphemes, words, and syntactic patterns.
} 
This is an argument against group markers as an explanation for the evolution of altruistic behaviour. It is indeed correct that the existence of such markers cannot explain why people are cooperative or, more specifically, altruistic. This, however, is a straw man. The word "cue" is also used misleadingly, which conflates two very different things. Kinship and previous behaviour clearly are cues to cooperation: once an individual has been identified as kin, or as reliable, then this is a cue to act cooperatively towards them. Cultural markers are also often referred to as cues, but they are cues to something different. McElreath et al. seem to be suggesting that they are a poor substitute for kinship and previous behaviour. This is missing the point. As argued above, it is not trivial to identify new individuals either as kin or as reliable cooperators, and this is precisely why social markers are useful. They are cues to kinship or potential reliability (on the basis of belonging to the same group). They are not, in other words, an alternative explanation for cooperation, but a means of reinforcing exactly those cues that McElreath et al. mention. Their second argument relates to green beards (122-3). Again, they are right that this is unlikely to work as an explanation for altruistic behaviour (see Section 2.2.1.1). However, given that the issue in question concerns cultural markers and that green beards are by definition genetic, McElreath et al.'s argument in this regard is strikingly beside the point.

McElreath et al.'s own argument is that "markers function to allow individuals to interact with others who share their social norms" (123), which they support with a mathematical model. This is convincing, and entirely reasonable. It is, however, far less different from Nettle and Dunbar's (1997) own view than they seem to be claiming. Both are arguing that markers allow the identification of conspecifics as members or non-members of one's own group, since it is beneficial to interact differently with the former than with the latter. McElreath et al. see the benefit as being about identifying other individuals likely to share one's own social norms, which allows one to maximise the payoff from the interaction. This, however, is still about cooperation, and it still assumes that the most beneficial cooperative interactions are likely to be with members of one's own group. The difference between the positions is that Nettle and Dunbar focus on avoiding outsiders who will exploit the interaction - the existence of whom, McElreath et al., for some reason, 
see as an argument against Nettle and Dunbar's position (128)—while McElreath et al. focus on avoiding outsiders who will misunderstand the norms governing the interaction. Not only is the difference small, but the two positions are eminently compatible. Indeed, as McElreath et al. note themselves (128), the social norms involved may well be moral norms concerned with preventing exploitation. In any case, it would be misleading to assume that the only purpose for cultural markers of group identity is to identify potential freeriders and cheats, and it would be entirely wrong to explain human cooperative behaviour simply on the basis of ethnic markers. McElreath et al. are right about the importance of markers to the maintenance of groups based on shared social norms, but are misleading in their suggestion that this is opposed to explanations that make reference to cooperation.

\subsubsection{Social selection}

A social marker, as noted on page 19, is useful to its bearer to the extent that it maximises fitness benefits to that bearer. This means being associated with the right groups and not with the wrong groups. We should thus expect variants of traits to be selected (whether consciously or unconsciously) on the basis of their social significance, which is not, it should be noted, inherent to the variant itself - at least in language - but comes about through association with individuals and groups of individuals. This process can be termed social selection (cf. Croft 2000: 32; Nettle 1999), although other terms are in use, such as Boyd and Richerson's (1985: 13271) more general (i.e. not specifically social) "biased transmission". They make a distinction between "direct bias", whereby an individual directly evaluates the worth of a particular cultural variant, ${ }^{10}$ "indirect bias", whereby the worth of a variant is based not on an evaluation of the variant itself, but on its selection by another successful individual, and "frequency-dependent bias", where the worth of a variant is determined based on the number of other individuals exhibiting it (135). It should also be noted that, in addition to such cultural selection of a variant by

\footnotetext{
${ }^{10}$ On some functional basis, for example. Although this does not necessarily mean that the cultural variant in question is actually valuable. The proximate explanation for why many animals gather shiny things is that they are innately biased to be attracted to them. There is an ultimate, adaptive, explanation underlying this, but that does not necessarily mean that every shiny thing must be of functional use to the animal that picks it up.
} 
an individual, a cultural variant may spread because it gives its bearers a reproductive advantage, and via this route gain a frequency-dependent advantage over other cultural variants (cf. Boyd and Richerson 1985: 227-40; Boyd and Richerson 1987; Soltis et al. 1995 on the related notion of cultural group selection). Of course, genetic evolution and cultural evolution should be expected to interact significantly. Individuals may, for example, select cultural variants because of the fitness advantage they confer. On the other hand, a cultural variant (e.g. contraception) may benefit from cultural selection, but lower the reproductive fitness of the individuals selecting it (for a discussion of gene-culture coevolution, see Laland 2002, 2008). The extent to which a trait can be directly selected by the bearer will also vary considerably, as discussed in Section 2.4.2. In any case, if a trait is selected for on social grounds, whatever the nature of the selection, this should affect its distribution. If this occurs in language (and, as noted at the end of Section 2.4.2, language seems to be an especially good source of social markers), then we should expect it to play a role in language change. Chapter 3 will discuss this in more detail. 


\section{Chapter 3}

\section{Change and variation in language}

Chapter 2 discussed the evolution of cooperation and argued that the use of social markers aids cooperative interaction, by allowing members of a group to quickly identify outsiders who may exploit the cooperative behaviour of group members, or, more broadly, whose social behaviour may be governed by different cultural norms. It was also argued that language - for reasons given in Section 2.4.2-is a particularly good source of such social markers. Given this, we might expect social factors to play an important role in language change, in particular in encouraging divergence into dialects. This is somewhat more controversial in linguistics than might be expected, however, and Section 3.3 will discuss the controversy. First, though, Section 3.1 will look at what precisely is meant by change and variation in language, and the relationship between them.

\subsection{The meaning of variation and change in language}

For social markers to exist, there must be variation - in language this means more than one way of saying the same thing. Since linguistic signs are arbitrary, there is in principle an infinite number of ways of saying the same thing. In practice this is limited by the number of acoustically distinct sounds producible by the human vocal tract (far from all of which are available for use in any given linguistic variety); however, there remains an enormous potential for variation in language, and this variation is the raw fuel of socially driven change; it is, indeed, a prerequisite of it. The process by which variation arises in language, however, is also usually called change. Obviously different things are meant by the word "change" in both cases. 
To be clearer about what is involved, it is worth probing the relationship between change and variation a little more deeply.

That change and variation in a communication system should be closely related is not quite a logical necessity. One can imagine a system in which variation remained entirely stable across time, or a population of robots whose identical communication modules all changed in unison. But in a culturally acquired system of the kind that we have, the two are inherently associated. What, however, is the relationship? It seems on first sight that they can be understood, respectively, as diachronic and synchronic reflexes of each other (cf. Milroy and Milroy 1985: 344). Understood this way, change is just diachronic variation. This seems to make some sort of intuitive sense, but it will not quite do. We would, presumably, also want to use the word to refer to the appearance of a new variant in some context, or its spread through a population. Here we do not seem to quite mean "diachronic variation" so much as the process that brings that variation about.

When we say that new variation has arisen, we also need to be clear with respect to what: one speaker or a population of speakers? On top of that, we must account not only for the appearance and spread of new variants, but also for the loss of old one, and shifts in their usage: the pejoration of "silly" and the lamentable loss of "neorxnawang" are evidently signs of change. Nor, finally, can we restrict ourselves to talking of changes in production, as some writers (e.g. Janda and Joseph 2003) appear to want to do. Underlying production is linguistic knowledge in the heads of speakers and, in being clear about what change and variation are, we need to be clear about how they relate to both production and competence. Sections 3.1.1 and 3.1.2 will attempt to define variation and change in a useful way for the purposes of this thesis.

\subsubsection{Variation}

First, variation should be distinguished from variability (cf. Section 2.4.2). A linguistic variable is any linguistic item that can vary, and a variant is a particular case of that variation. The level or domain in which a variable is to be found is dependent on context and does not form part of the definition. For example, one might treat the concept of [MEAT] as a variable with, as its variants, words for meat 
in various languages, whether represented orthographically or phonemically: meat, мясо, cig; /mit/, /'m ${ }^{\mathrm{j}} \mathrm{aso} /, / \mathrm{kig} /$. Alternatively, one of these words, say the free morpheme $\{$ meat , might be considered a variable with such phonetic variants as [mit], [mi?], [mit], [məri].

There will usually be a context or set against which this is to be considered, and that depends on the particular research question. A researcher may be interested in all the words for [MEAT] in the world's languages, but may just as well be interested in the phonetic realisations of the word (or one of its phonemic segments) in a particular monolingual corpus, or even a single conversation. These are all cases of variation in production. A speaker may equally well be interested in looking at variation in the competence of a particular speaker, or in the grammars of a particular population.

This leads us to a definition of variation:

The existence of more than one variant for a given variable.

It will be noticed that no distinction is drawn between synchrony and diachrony. For sets of utterances at least, this is partly because a principled distinction is rather hard to maintain. Research on dialogue has consistently shown that speakers align to each other over the course of a conversation (Bock 1986; Branigan et al. 2000; Pickering and Garrod 2004, 2006; Pardo 2006); is a corpus drawn from a single conversation thus diachronic? Then what is left that can be considered synchronic? How short, in other words, must a time-window be to be considered synchronic, and how long before it becomes diachronic? There seems less of a problem in the case of competence: it is easier to imagine a snapshot of the linguistic variants stored in a set of speakers' brains at a particular moment than a split-second snapshot of those speakers' utterances. This is because the brain is a physical space, in which we imagine information to be in some sense all present at the same time. Spoken discourse, on the other hand, consists very obviously of sounds spread across time, and is thus inherently diachronic (as is signed discourse). ${ }^{1}$ We must be cautious: this view of brains may simply reflect greater naïvety about the nature of mental representations. Moreover, the means currently available for investigating mental

\footnotetext{
${ }^{1}$ Written texts are a little different, but, like spoken texts, are accessed diachronically.
} 
representations of linguistic knowledge do not allow us to simply take such a snapshot in the way we might like. Nevertheless, it seems reasonable for the time being to refer to synchronic and diachronic variation with respect to linguistic competence. With respect to production, it seems we are obliged either to talk simply of variation, specifying the set of utterances involved, or to distinguish synchronic from diachronic variation, specifying the length of the relevant time-windows.

\subsubsection{Change}

Variation was defined above (page 29), relatively straightforwardly, as "the existence of more than one variant for a given variable". Change is more troublesome. For example, it has been taken for granted so far that language change is an indisputable fact. Ritt (2004: 11), however, seems to express doubt about this very thing:

Of course, it deserves to be stressed right at the outset that the very claim that languages actually "change" is not really self evident. "Language change" is no "fact", but a theoretical construct because processes of "language change" are not observable as such. That language change is nevertheless treated as if it were a "fact" reflects, basically, a complex but plausible interpretation of observable variation among linguistic texts from different historical periods.

The point, which has been raised in other places (see e.g. Andersen 1989: 12-13; Janda and Joseph 2003: 13), is that change and diachronic variation are not quite the same thing after all (or, if diachronic variation is to be called change, then it is only one of several things referred to by that name). Indeed, Janda and Joseph (2003: 13) advocate a three-way distinction between "diachronic correspondence", "innovation", and "change". The first refers to a comparison of two linguistic states, separated by time; the second to an event initiated by a particular speaker at a particular time; and the third to a process "requiring adoption, over time, by all - or at least much - of a group." Change in this paradigm, in other words, is specifically change on the level of a population of speakers, or grammars; it is a change in the distribution of variants, and is kept distinct from change in a particular speaker's grammar, which, if it fits into this framework at all, must come under innovation (although the definition seems to refer specifically to production). These distinctions are vital ones to make, and this narrow use of the term "change" 
at least accords well with what most people, particularly non-linguists, first think of when they hear the term "language change" - given an understanding of the term "language" as something like the combined linguistic knowledge of a statistical clustering of speakers (see Section 3.2).

However, this division still has the potential to mislead, partly because "change" is still a very broad term in common usage - an innovation is clearly a kind of change, in the normal sense of the word, for example - and the word is hardly used more precisely even in the field. Indeed, Croft (2000) quite reasonably interprets Joseph's (1992: 127) claim that "language change always takes place in the present, i.e. it always occurs in some speaker's (or group of speakers') present" as meaning that "only innovation is a language change" (Croft 2000: 5), a claim that not only employs the term "change" differently from Janda and Joseph (2003), but implies that the latter's usage is unhelpful. Croft cites Milroy (1992) and Labov (1982) as holding the opposite view: that a change is not a change until it has been propagated. Similarly, Andersen (2006: 67) asserts that, "Traditionally linguists conflate the notions of innovation and change and name different types of change by the kinds of initial innovation that gave rise to them."

A desire that the word be used in the narrow sense that Janda and Joseph want thus seems a little quixotic. The best response, it would appear, is to allow "change" to be used in a somewhat more overarching sense (see below), and to make distinctions elsewhere. One distinction in particular is worth being clear about. This is between what Croft (2000) prefers to call innovation and propagation (terms that have the advantage of already being in common usage in linguistics, and of being relatively straightforwardly applicable to complex systems other than language). The first he uses to refer to the "creation of a novel variant" (238) and the second to refer to diffusion of a variant (in the case of language, this usually means from speaker to speaker, but should also be taken to include diffusion between stylistic contexts; see Kroch 1978; Moore 2004). ${ }^{2}$

\footnotetext{
${ }^{2}$ Croft specifically defines propagation as "the increase in frequency of a lingueme [i.e. a variant] in a language by selection." (241) This definition seems to exclude the spread of variants by drift, but this is more a reflection of Croft's views of how propagation tends to occur than a part of the definition; elsewhere he allows drift as a "relatively minor propagation mechanism" (62).
} 
Croft does not, of course, claim to introduce the distinction, which has a long history in linguistics; Weinreich et al. (1968), for example, use the terms "actuation" and "diffusion" (to which Croft also refers, as alternatives) in a very similar way. "Innovation" is perhaps preferable in its transparency to "actuation" (although the term "actuation problem" is now well established), but there seems little to choose between propagation and diffusion. In any case, the distinction is a very useful one, which will be maintained here (see also Section 3.1.2.2). Any instance of change must involve at least one of the two.

But this still leaves open the question of what is meant by change. A commonsense understanding is that if there is a difference between the state of an object at two different points in time (i.e. diachronic variation, or "diachronic correspondence", to employ Janda and Joseph's usefully more specific term), then we can infer that change has occurred. For example, if a house has a blue front door on 1 January 1989 and a red front door on 31 December 1990, then we can say that the colour of the door has changed at some point during 1989 or 1990 . We can also say that the door has changed, that the house has changed and that the world has changed. Of course, even if the door is blue at the end of 1990, there might still have been a change - the door might have been painted red and then repainted blue. In any case, the key issue, as Ritt (2004: 11; quoted above, page 30) implies, is that in this example we have inferred the change rather than observed it - the existence of the two states separated by some period of time is not itself a change. "Change" is rather a term we apply to whatever activity led from one state to the other: in this case, it is something that starts when the old coat of paint is removed (if it is removed) and ends when the new coat has dried, and the desire to be more precise about what exactly it is that counts as change is akin to the desire to be precise about when a collection of grains of sand becomes a pile. Ritt's point should not be taken too far, however: it would be excessively pedantic to seriously deny that change occurs simply because it is inferred rather than directly observed. The explanandum, strictly speaking, is the fact that there is diachronic variation in language. Theories of change attempt to explain how and why a move from one state to another occurred; this is as much an explanation of language change as of linguistic variation in time. 
Language change, then, can be defined as:

The process of moving from one linguistic state to another.

The next sections will expand on this. Sections 3.1.2.1 to 3.1.2.3 will elaborate on important distinctions; Section 3.1.2.4 will provide a broad taxonomy of language change.

\subsubsection{Change and diachronic variation}

Based on the above discussion, it seems we can say that the practical difference between diachronic variation (or diachronic correspondence) and change is primarily one of emphasis. The former term emphasises the difference between mental states, or utterances, or populations of speakers, at different points in time, while the latter emphasises the movement from one to another. The real difficulty is in clearly specifying what has changed: moving from one state to another of what? Some answers are straightforward. With regard to individual competence, we can say that a speaker's grammar has changed, or that their brain has changed. If a new word has been learnt, we can say that a lexicon has changed. With regard to a population of grammars, we are interested in a change in distribution: the proportion of grammars that have a particular pattern of variants.

We must be specific about what constitutes a population, of course. If we compare the same people in 1970 and 2000, then this is straightforward (even if the practicalities of finding them are not); if we compare speakers in 1623 and $1923,{ }^{3}$ however, then we must be clear about how we are selecting the two sets of speakers. Perversely, for there to be change, something must remain constant. For English speakers in one century to be compared with English speakers in another, and for us to able to say that English has changed between the two centuries, there must be something constant that we call English (which is precisely why Janda and Joseph 2003 use the term "diachronic correspondence"). This is not the place for an examination of the Ship of Theseus paradox - lt is enough for present purposes to be aware of the issues - but there is one further point worth making. Some differences

\footnotetext{
${ }^{3}$ It will be objected that in both cases we are actually comparing linguistic output rather than grammars. The point is that even with contemporaries, grammars can only be inferred from output. We are simply more limited with historical speakers in our methods for inferring grammars.
} 
between a corpus from 1623 and 1923 may be significant, but others may simply reflect the variation that is to be expected between two corpora and which is not statistically significant. Here there appears to be a distinction in intuition: where the difference between two things separated by time is statistically insignificant, it seems clearer to talk of diachronic variation; where the difference is significant, one feels more justified in talking of change.

\subsubsection{Innovation and propagation}

Innovation and propagation were defined above (page 31), and the difference between them can be stated clearly: propagation occurs between speakers, and can be defined as the transmission of information from one grammar into another (which occurs via production). Innovation, on the other hand, can only occur in a single speaker, not in a population. ${ }^{4}$ There is, granted, one terminological issue here, which can be summed up in this question: can innovation in a grammar be distinguished from grammar-level change (as distinct from change in production)? If not, then innovation refers both to a change in a grammar that has its origins in that grammar and to the result of an instance of propagation. If the two can be distinguished, then innovation is a subset of grammar-level change, and refers only to such changes as originate in the grammar. ${ }^{5}$ Since such a distinction-between innovation and grammar-level change - is likely to be useful, it will be maintained in this thesis.

\subsubsection{Production and competence}

A further distinction, which has a very long history in linguistics, is that between competence and production: between the linguistic information in a speaker's head - their grammar and lexicon - and the utterances the speaker produces. ${ }^{6}$ In

\footnotetext{
${ }^{4}$ Of course, we tend not to be aware of innovations until they have already spread to many grammars. Therefore we might talk of an innovation "in a dialect of English"; this is because we do not know the particular source of the innovation (and there may be several), but also because the innovations that tend to matter to us are the ones that propagate (cf. Milroy and Milroy 1985: 348).

${ }^{5}$ Such changes can, of course, come about as a result of propagation, where imitation is less than perfect.

${ }^{6}$ This is not quite the same as Chomsky's (1986) distinction between I-Language and ELanguage. The former is equivalent to competence, but the latter is essentially a term for everything else that might be referred to as language, and which Chomsky does not find to be a coherent concept.
} 
the context of change, however, they are not as easy to distinguish as might be expected, since what is called change in production tends to be simply a reflection of a change in the grammar or lexicon. Now, inasmuch as two instances of linguistic production from one speaker, separated by time, can differ, it does not seem unreasonable to speak - at that level of analysis - of change in that speaker's production, just as we talk of a change in the colour of a door, when we know that, really, the change is in the particular chemical composition in the coating on the door, and the colour difference we perceive is a product of that. There can, however, be change in a grammar that is not reflected in production, simply because the relevant discourse context does not arise that would allow the change to be revealed. At least we can assume such changes are possible; they are, by definition, unobservable by the methods currently at our disposal.

Innovation in production can also occur that does not reflect any change in the grammar; a slip of the tongue is a familiar example. Can we speak, however, of propagation in production? First, it should be noted that linguistic production is the only means by which propagation occurs. In a simplified example, a speaker says something (the form of which, slips of the tongue aside, is constrained by their grammar); another speaker hears this and, perceiving something novel (whether consciously or unconsciously), makes some change to their grammar. ${ }^{7}$ In this case, if the hearer then reproduces the novel variant, this simply reflects propagation between grammars, not production.

So can propagation occur in production, but not between grammars? We might consider the case where a speaker produces a variant that is not encoded in their grammar (presumably as a production error), and this variant enters the grammar of another individual. Here there is at least propagation from production to grammar, not from grammar to grammar via production. How about propagation to production? This could occur if a speaker were to imitate something that another

\footnotetext{
${ }^{7}$ This is, of course, greatly simplified and, to an extent, theory-dependent. In a more exemplartheoretic account, every variant heard would be stored mentally - counting, trivially, as a changeand, eventually, changes in the proportion of variants might lead to a more significant shift in the grammar. In any account, we should certainly not expect every novel variant to induce a significant change in the hearer's grammar; there must be something more involved, be it frequency, prestige, or some other biasing factor.
} 
speaker said, but without storing it in their grammar. ${ }^{8}$ If this is an imitation of a production error, then we have propagation from production to production, via the speakers' brains, but plausibly not via their grammars.

\subsubsection{Kinds of change}

A movement from one linguistic state to another can take four forms. ${ }^{9}$ It might involve:

(a) the introduction of new items (e.g. a new word for something, or a new speaker);

(b) the removal of items (e.g. the loss of a past-tense form, or the death of a speaker);

(c) the replacement of one item with another (i.e. the simultaneous occurrence of a and $\mathrm{b})$;

(d) the reorganisation of items, where an already-existing item takes on some new role, or the relationship between items changes (e.g. euphemism, where a phonetic string expands in scope to refer to a new referent).

While these four categories are most obviously applicable to innovation, where the "item" is some linguistic variant, they also apply to propagation. Change at the population level can also be discussed in the same terms - the unit in this case would most often be the grammar, or speaker.

\subsection{Convergence and divergence}

Language change invariably leads speakers to become less or more like each other. ${ }^{10}$ In terms of the kinds of change discussed in Section 3.1.2.4, this is a reorganisation

\footnotetext{
${ }^{8}$ Again, this understanding depends somewhat on our understanding of what constitutes a grammar. In an exemplar-based model, then speakers might store everything they hear, albeit in some cases fleetingly, in their grammar.

${ }^{9}$ Andersen (2006: 67) defines innovation in much this way (though in broader terms), as "(a) the introduction by a speaker ... of a new variant, or (b) the modification of an existing variation", yet mysteriously neglects the removal of variants (unless this is considered to be included under "modification").

${ }^{10}$ In fact, it should be noted that the degree of difference between two speakers may well depend on the context in which they are speaking. Intra-speaker variation, whether defined in terms of dialect or style (a distinction Moore 2004 is in favour of abandoning) is ubiquitous, and has been long recognised (e.g. Trudgill 1974; Kroch 1978). A particular case of this-alignment between two speakers in dialogue - will be discussed in more detail in Section 3.3.1.2. An extreme (though also unexceptional) case of such variation is multilingualism, and the evolutionary context to this will be discussed in Section 10.3.
} 
change (type d) on the level of a population; on the level of the speakers' grammars, on the other hand, other kinds of change are involved.

When speakers become more like each other, this is called convergence, and it represents a reduction in variation; the opposite - when speakers becomes less like each other - is divergence, which represents an increase in variation. Neither process, nor the relationship between them, is quite as simple as it may seem (cf. Giles et al. 1991). Consider the relationship between Latin and the modern Romance languages: these languages are quite reasonably perceived as having come about through a process of divergence. Nevertheless, on the level of individual speakers and groups of speakers, there must have been convergence involved as well. If the history of Romance speakers from the fall of the Western Roman Empire to now could be described entirely in terms of divergence, then we would expect there to be an extravagant multitude of Romance idiolects, not the relatively manageable collection of languages and dialects that we in fact see. Divergence leads to a spreading out across linguistic space, and convergence to a coming together at some point on it; a combination of the two leads to statistical clusters in the space, and it is these, on the whole, that get called languages and dialects (see Croft 2000, 13-20, for a related discussion in evolutionary terms). ${ }^{11}$

The development of Latin (or, more accurately, Proto-Romance) into the modern Romance languages, in other words, was the divergence of one statistical cluster into several. The actual movement in linguistic space, however, was done by individual speakers (replaced periodically through birth and death), and this involved both divergence and convergence. The relationship between Ancient Greek and Modern Greek, by comparison, is of a different kind. Here, several clusters converged into one, and this must have involved convergence between individual speakers. Strictly speaking, koineisation of this sort need not involve divergence between speakers, although in practice one can assume that at least some divergence between individual speakers will inevitably occur.

\footnotetext{
${ }^{11}$ The distinction between the two is famously fuzzy, and may involve political factors. Of two clusters of similar density, for example, one may be treated as representing one language, while another may be treated - for political reasons - as representing two or more.
} 
Convergence is mainly driven by the propagation of variants between speakers. ${ }^{12}$ Divergence between members of a speech community, on the other hand, must involve either innovation by members of the community or the arrival of new variants from outside it. In any case, something new must be introduced. However, as noted above, if divergence is to mean divergence into dialects and not idiolects, there must be convergence involved, and thus propagation within the community. This propagation must in some way be biased; if it were not, then variants would propagate equally between all members of the community, and speakers would end up neither closer to each other nor further apart. The simplest such bias is based on frequency of interaction, which may simply be the result of geographical distance between speakers (including the presence of natural barriers that hinder travel). On the other hand, there may be some other bias involved: variants may be more likely to propagate between some speakers than others, and the reasons for this are likely to be social, involving such specific factors as prestige and identity.

\subsection{Social factors in language change}

It is well recognised in linguistics, and has been for a very long time, that language is affected by social factors. The field of sociolinguistics, after all, is primarily concerned with the association between social variables and linguistic ones. Although the field had its origins in work on dialectology that began in the nineteenth century, modern sociolinguistics owes a considerable amount to the pioneering work of William Labov, and one of his earliest articles, his 1963 study of sound change in Martha's Vineyard, is still cited both as a foundational paper for the field (see e.g. Meyerhoff 2006; Pope et al. 2007) ${ }^{13}$ and in support of the claim that social factors play a significant role in language change and divergence (e.g. Nettle and Dunbar 1997: 93). This study showed that the use of certain phonetic variants

\footnotetext{
${ }^{12}$ It may also involve homoplasy, and in some cases this is quite likely. As historical linguists have been very well aware for a long time, intervocalic voicing need reflect neither common ancestry nor borrowing.

${ }^{13}$ As Meyerhoff (2006: 20) points out, this study was almost, but not quite, the first study to find evidence for the involvement of social, as distinct from geographical, factors in language variation.
} 
by inhabitants of Martha's Vineyard correlated significantly with attitudes to outsiders, especially the rich visitors who would spend their summers on the island. ${ }^{14}$ Fishermen selected centralised variants of the (ay) and the (aw) diphthongs, apparently unconsciously, which were associated more with traditional island speech patterns than with the outsiders. In Labov's words: "the meaning of centralization ... is positive orientation towards Martha's Vineyard" (306; italics original). Blake and Josey (2003) carried out a follow-up study on the island forty years on, which suggested that, at least with regard to the (ay) variable - the focus of their studythe situation was no longer as Labov had found it. In contrast, work by Pope et al. (2007), which was a closer replication of the original study, found similar results to Labov, especially for the (aw) variable, although they also found that change had slowed for the (ay) variable.

Labov's study is a classic work that has had a huge impact on the field, and which seems to provide clear evidence for social selection at work in language change. However, a fact often forgotten or ignored is that Labov himself is cautious of how broadly it can be applied:

The Martha's Vineyard study ... is frequently cited as a demonstration of the importance of the concept of local identity in the motivation of linguistic change. However, we do not often find correlations between degrees of local identification and the progress of sound change. (Labov 2001: 191)

He states, moreover, that:

It does not seem likely that the linkage between linguistic and social structure involves the association of forms and frequencies with particular groups and calculations of the consequences of adopting their speech forms ... [T] he simpler and more mechanical view [is] that social structure affects linguistic output through changes in the frequency of interaction. (ibid., 506)

\footnotetext{
${ }^{14}$ Hostility towards holiday-home owners, who are often able to buy locals out of the housing market, and who tend not to contribute to the economy throughout the year, is not restricted to Martha's Vineyard. In Wales in the late twentieth century, holiday homes were burnt down by a group called Meibion Glyndŵr, and legislation has since been passed in Gwynedd to control the number of holiday homes. There is similar hostility in other parts of the UK, and in Europe more widely. The stereotypical holiday-home owner - an outsider who enjoys the best of an area, but gives little in return - is a classic freerider.
} 
Labov refers to the primary importance of frequency of interaction as the "principle of density" (ibid., 19-20), according to which most cases of the propagation of linguistic variants are explicable in simple, mechanistic terms.

\subsubsection{Trudgill and new-dialect formation}

Peter Trudgill, another highly significant figure in the development of sociolinguistics, is of the same view, and he applies it to his explanation of the emergence of new dialects in colonial contexts (see e.g. Trudgill 1986, 2004, 2008a). His claim is that the emergence of new colonial dialects is the result of mixing, mediated by accommodation in face-to-face interaction. The emergence of new dialects is thus, in line with Labov, "inevitable" (Trudgill 2004) and mechanistic, mediated by accommodation to one's interlocutor - which Trudgill views as an unconscious, automatic process - and thus ultimately by the frequency of interaction between interlocutors. Although Trudgill admits that identity plays a role in some sociolinguistic situations (2008a: 243), he claims it plays no role in the formation of new colonial dialects - indeed, that it is simply "not ... powerful enough" to explain the new dialects, but, rather is "parasitic upon accommodation, and is chronologically subsequent to it" (ibid., 251-2).

This stance has much to recommend it: if accommodation occurs automatically in ordinary discourse (though see Section 3.3.1.2), then an explanation that can account for language change in terms of it, without invoking identity or speakers' attitudes in general, is clearly a more parsimonious one, and there is broad support in the field for privileging more mechanistic accounts. Croft (2000: 78) makes a similar, though not identical, point when he states that "a good methodological strategy would be to seek nonintentional mechanisms first, and only turn to intentional mechanisms if those fail". As Mufwene (2006; 2008) points out, moreover, Trudgill's account is also compatible with research into the development of creole languages. Both he and Trudgill make reference to the uniformitarian principle (Mufwene 2008: 254-5; Trudgill 2008a: 250-1): if identity cannot be invoked in some cases of creole-formation and colonial dialect mixing, then there is no reason to invoke it in other cases. 
This all seems compelling, but Trudgill's claims have nonetheless been challenged. His deterministic account of the emergence of New Zealand English (Trudgill 2004; see also Gordon et al. 2004) prompted Baxter et al. (2009) to present a mathematical model contradicting it. His 2008 a paper attracted six responses in the same journal issue (Mufwene 2008; Tuten 2008; Schneider 2008; Coupland 2008; Bauer 2008; Holmes and Kerswill 2008), along with a rejoinder by Trudgill himself (2008b). That the issue should attract such a significant response should not be surprising. It certainly has great bearing on the central question of this thesis, which is why it will be discussed in some detail here. While it is obviously a question of great importance to the study of change and variation in language, it is also relevant to the psycholinguistic study of dialogue (whence the concepts of accommodation and alignment; see Section 3.3.1.2). Since it is relevant, moreover, to models of cultural evolution and language as an example of a complex system, it is of interest to biologists, physicists and mathematicians (see e.g. Atkinson et al. 2008; Baxter et al. 2009). With regard to Trudgill's own field: as Coupland (2008: 267) notes, there has been a tendency in sociolinguistics for identity to be the "first explanatory resource", and, even among critics of his 2008a paper, Trudgill finds much support in his rejection of this knee-jerk tendency (e.g. Mufwene 2008; Tuten 2008; Schneider 2008; Coupland 2008); the same point has also been made elsewhere (e.g. Meyerhoff 1998). Mufwene (2008: 257) and Holmes and Kerswill (2008: 275) also side with Trudgill in recognising the implausibility of "guided" evolution in this contextof speakers early in the process of new-dialect formation explicitly setting out to create a new speech community, or deliberately developing new vowel qualities, which Trudgill (2004: 157) rightly calls "ludicrous". ${ }^{15}$ But this is something of a straw man, and hard not to concede: no one would seriously argue that linguistic divergence and dialect formation are generally best explained by such conscious and deliberate activity. Similarly, Coupland (2008) agrees with Trudgill in his rejection of people of disparate origins coming together in some sort of identity pact. This is also rather a straw-man argument, however. Trudgill (2008a: 252) cites Keller's (1994: 100) maxim of, "Talk like the others talk", as an expression of

\footnotetext{
${ }^{15}$ Although there are certainly several historical cases (such as in America and the Balkans) of members of speech communities explicitly seeking to promote linguistic variants associated with their community in contrast to another. See Section 9.2.1.
} 
the basic drive to accommodate. Keller's focus in his book, however, is on the largescale unintended consequences of small-scale intentional actions - which he calls "epiphenomena", or "phenomena of the third kind" - making reference, in the title of the book, to Adam Smith's "invisible hand" in economics. The role of identity in such a large-scale phenomenon as dialect formation should not, therefore, be lightly dismissed on the grounds that large-scale intentional coordination by speakers is implausible.

\subsubsection{Claims and criticisms}

Before looking at the criticisms of Trudgill's thesis, it would be as well to look at what precisely he is saying. He is really making three arguments:

1. That automatic, unconscious accommodation is sufficient to explain the emergence of new dialects, and identity need not be invoked;

2. That national identity cannot be invoked before the 16th century, so should not be invoked later either;

3. That identity is not a powerful enough factor to explain the emergence of new dialects.

The third of these is particularly poorly motivated, and Trudgill's argument does not go far beyond the statement that "identity factors cannot lead to the development of new linguistic features" (2004: 157; 2008a: 251). Be that as it may, this is not the point, as he admits:

This is, of course, not necessarily the same thing as saying that once new linguistic features have developed they cannot BECOME emblematic. (Trudgill 2008a: 251, emphasis original; cf. Trudgill 2004: 157)

He is right to call the idea that speakers are deliberately creating new dialects from scratch ludicrous (Trudgill 2004: 157), but that is not what is being claimed. The role identity is assumed to play is not in innovation, but in propagation ${ }^{16}$ (cf. Baxter et al. 2009: 269). Trudgill may mean that identity is not powerful enough on its own to lead to change, but must work together with accommodation, or

\footnotetext{
${ }^{16}$ Although that is not to exclude it as a possible source of innovation. An attempt to imitate a particular accent for social reason might "overshoot" and lead to the creation of a new variant.
} 
some similar process. If so, what is the alternative that he objects to? Surely no one seriously proposes that identity leads to language change unmediated by any other process. The real question is whether social factors can play a significant role at all and influence how accommodation occurs. This brings us to his first two arguments, which are more clearly based on empirical foundations than his third. These are the main focus of criticism in responses to his 2008 paper, of which there are essentially four:

1. That his conception of identity is too narrowly focused on large-scale national identity (Tuten 2008; Schneider 2008; Coupland 2008; Holmes and Kerswill 2008).

2. That his invocation of the uniformitarian principle puts the cart before the horse in seeking to explain general processes with reference to poorly understood historical case studies (Schneider 2008; Holmes and Kerswill 2008)

3. That accommodation cannot lead directly to language mixture (Bauer 2008).

4. That the processes Trudgill invokes rely themselves on social factors (Schneider 2008; Coupland 2008; Holmes and Kerswill 2008).

Not all these criticisms are overwhelming. Bauer argues, for example, that accommodation "cannot lead directly to a mixed dialect ... because different speakers will accommodate to different degrees, and, in any case, will accommodate to the interlocutor of the moment" (272). This is true, and the possibility of variation in levels of accommodation is a highly important one, that will be discussed in more detail below. His point about accommodation in the moment is less convincing, however, even given that he is certainly right that this will occur and will not - on its own - lead to dialect mixing. Of course accommodation cannot lead directly to dialect mixing; of course there must be some influence on the speaker's grammar. It seems clear, however, that such an influence is assumed in Trudgill's model; indeed, he makes explicit reference elsewhere to usage-based models, in which frequency plays an important role in grammar change (Trudgill 2004: 150-1) ${ }^{17}$, a fact not missed-perhaps unsurprisingly, given the authorship-by Baxter, Blythe, Croft and McKane (2009).

\footnotetext{
${ }^{17}$ See Pierrehumbert (2001); Bybee (2006, 2007); Bod et al. (2003) for a review of such approaches.
} 
The criticism of Trudgill's appeal to uniformitarianism is more interesting. The point of the uniformitarian principle is that, in seeking to explain historical eventsfor which we may lack data - we are best served by relying on processes with which we are familiar through observing recent events, for which we have more data. It is thus an application of Occam's razor: we explain the unknown as far as possible with reference to those entities we already know to exist. Trudgill's argument, however, is that there are examples to be found of new colonial dialects developing before the 16th century, the main period of European colonial expansion, and that there is no evidence that national colonial identity factors played a role in these cases. Nor should we even expect them to have done so. Since we do not invoke national identity in our explanations of these cases, he argues, we should not do so in other cases. Schneider (2008) is right to find this an odd application of the uniformitarian principle, but it is odd only because we generally know less about more distant historical events. However, if Trudgill is right that we know enough to rule out national identity in the older cases, then it is indeed more parsimonious to assume that it is unnecessary for explaining the later cases, assuming the newer and older cases are indeed comparable in relevant details. And the rejection of national identity as a significant factor in the pre-16th-century cases does indeed seem reasonable; the genesis of nationalism is, after all, usually located in the late eighteenth century, around the time of the French and American revolutions.

Much hangs on the word national, however, and this is the clearest criticism. There is, in short, more to identity than this; indeed, to quote Coupland (2008: 269): "identity is often less coherent, less rationalized, more elusive, more negotiated, and more emergent than this." It is not as if, prior to the development of nationalism, identity was not an important factor in human social psychology. It is simply that the association of an individual with a nation-state, rather than a city or other smaller-scale entity, developed at this point. Nor will it do to protest that Trudgill is really talking only about national identity, even if this is all he refers to directly. He is clear in his reference to the automaticity of accommodation, and he is clear in his claim that this (mediated by frequency of interaction) is sufficient to explain colonial dialect formation, that he is rejecting all forms of identity, and indeed social factors more generally. Given this, his argument concerning pre-16th-century cases 
seems rather redundant. It is presumably intended to provide empirical support for the parsimony-based claim. But, as noted, it does not go far enough. It suggests only that new dialects can form without large-scale national identities, which is to ignore the possibility of other sorts of identity factors (cf. Holmes and Kerswill 2008). As Tuten (2008: 259) states:

newly arrived inhabitants often faced common human and natural threats [that] could well ... favor the development of a "frontier" identity in opposition to local "others" ... as well as to distant "others" who remained in home communities.

The emphasis on some sort of explicit, coherent large-scale identity is simply misleading (again it seems that Keller 1994 is being ignored; see page 42). It is enough that the new arrivals identify more, on an individual level, with other new arrivals - or with some of them - than with people they left behind, and this is precisely what one would expect, particularly in the kind of frontier zones Tuten refers to. They may still identify as nationals of their native lands, but that does not mean that they identify as much with the people they left behind as with people they now mix with. To put it another way, a bias does not have to be huge to have a significant effect over time (cf. Kalish et al. 2007). Similarly, Holmes and Kerswill (2008: 276) point out, with reference to Trudgill (2004), that:

Rather than searching for evidence of the development of a "New Zealand" identity at the early stages of dialect mixing, we should be looking for evidence on what it would mean to sound (locally) acceptable within any particular new, socially diverse community.

So this argument is not overwhelming. Trudgill's best argument was always about parsimony - that identity is simply unnecessary to explanations of dialectformation. It is self-evidently more parsimonious to explain any phenomenon in terms of automatic, universal mechanisms. There is a false dichotomy here, however. Trudgill (2008a: 252) makes reference to the biological, adaptive, basis of accommodation as evidence for its being a powerful, automatic, universal tendency. This seems to imply that social factors have no such biological basis (Holmes and Kerswill 2008 explicitly make the same distinction: 275). Yet, as I hope to have shown in Chapter 2, there is good reason to suppose that the drive to mark one's 
membership or non-membership of a particular social group also has an adaptive, biological basis.

\subsubsection{Accommodation and alignment}

There is another false dichotomy in the implication that explanations based on accommodation and frequency of interaction exclude social factors. There are two ways for this to be false: social factors may be involved, but relatively unimportantly, or they may be integral to the process. As an example of the first case, let us assume a neutral model, as Trudgill does, in which dialect-formation is simply the result of accommodation to all interlocutors and variation in the frequency of interaction. As Schneider (2008: 264) suggests, this variation in the frequency of interaction is likely to be affected by social factors: the groups to which individuals belong, and which shape the frequency with which they interact, are formed on the basis of social factors related to identity. In other words, we interact more with those we identify with more strongly. But the influence of social factors on accommodation here is indirect and relatively uninteresting. The more interesting second case is where social factors exercise a direct influence. There are various ways in which this might plausibly work: for example, speakers might accommodate more to people they are keener to associate with; or they might accommodate more to all interlocutors in certain socially marked situations (where, for example, nervousness or uncertainty plays a role).

Indeed, it has long been recognised in Speech Accommodation Theory (SAT) and its broader successor, Communication Accommodation Theory (CAT), that speakers both converge and diverge (Scotton 1985 makes a distinction between accommodation and "disaccommodation"), depending on attitudes. Bourhis and Giles (1977) and Bourhis et al. (1979), for example, are classic experiments that demonstrate accent divergence. As Giles et al. (1991: 11) note, moreover, "basic convergent-divergent shifts are ... not as descriptively simple as they might at first appear." Various sub-categorisations can be made, depending on the social relationships between the individuals involved, the relative degree to which they converge or diverge, and variation in the linguistic dimensions on which this occurs. It has also long been known that convergence may be to a perceived, rather than 
an actual target (e.g. Cohen and Cooper 1986; Giles and Wiemann 1987), or may converge on a related - and easier, or more acceptable - target, rather than the patterns of their interlocutor (Seltig 1985).

In other words, linguistic accommodation has, throughout its theoretical history, been assumed to be influenced by identity and social relationships between the speakers involved (cf. Coupland and Jaworski 1997; Coupland 2008; Meyerhoff 1998). As Coupland (2008: 269) states:

When Trudgill writes that "accommodation is not only a subconscious but also a deeply automatic process," he is challenging the fundamental claims of the model.

All the same, there is not complete agreement on this, and Tuten (2008: 260), for example, agrees with Trudgill that accommodation is "largely automatic and mechanistic". He makes reference to Pickering and Garrod's (2004) Interactive Alignment Model, and notes that Trudgill (2008a) does not make reference to this himself. This is indeed surprising, for it is an important assumption of this model that the process is largely automatic. It is, moreover, not generally assumed to occur as a means of signalling identity, but "as a mechanism to promote mutual understanding between interlocutors" (Pickering and Garrod 2004: 188). Since it is concerned with establishing common ground in interactions, and is achieved through priming (Bock 1986; Branigan et al. 2000), interactive alignment differs from accommodation in certain respects: the model does not easily include the possibility of alignment to an imagined target, for example. There is, however, an important qualification to be made about its automaticity. Pickering and Garrod (2004) argue, with reference to Bargh (1989) that alignment most likely occurs at a "postconscious" level. Crucially, this allows the possibility of its being influenced by external factors:

In general, we expect that rate of alignment may be affected by social factors even when the interlocutors are unaware that they are aligning. (Pickering and Garrod 2004: 214)

There is evidence, for example, that a desire to affiliate with the interlocutor can enhance behavioural mimicry (Lakin and Chartrand 2003; Lakin et al. 2008), 
while Branigan et al. (2007) show that alignment can be affected by the prior conversational role of participants.

There is nothing, therefore, about models of accommodation and interactive alignment that specifically excludes the role of social factors. Again we return to Trudgill's best argument: these models provide a framework that allows the exclusion of external factors. So if dialect formation can be accounted for without reference to such factors, then it is self-evidently more parsimonious to do so. Trudgill (along with Labov, Meyerhoff, and others) is right that this should be our default assumption; even if identity is involved somewhere along the line, it may form an unnecessary part of our explanation. What remain are, first, the smallerscale empirical questions of whether identity is relevant to particular cases of dialect formation and, second, the broader question of what the typical pattern is - what a generalised model should include. Baxter et al.'s (2009) mathematical model, as it happens, suggests that identity is in fact a necessary element in accounting for the particular case of New Zealand English.

\subsection{Conclusion}

Interactive alignment and, more broadly, communicative accommodation are models that allow a mechanistic explanation of convergence and divergence in language. At the level of interaction between individual speakers, and in combination with usage-based theories that allow language change to occur in adult speakers, based at least partly on token frequency (Miller 1994; Croft 2000; Pierrehumbert 2001; Bod et al. 2003; Bybee 2006, 2007), these frameworks allow the propagation of variants to be modelled theoretically, and can account - to an extent - for differential propagation through variation in the frequency of interaction. However, they do not exclude the possibility of social factors influencing propagation, and we might expect these, on the basis of the discussion in Chapter 2, to play a role especially in cases where identity is of particular importance and has some relevance to survival and economic success. Chapters 5 to 9 of this thesis will present an experimental model investigating this very question. In the meantime, there is a second, more theoretical, question that arises from the discussion of language change in this chapter. While this is less essential to the main argument of this thesis, it is important 
to the understanding of how social factors might influence language change: if there is socially biased selection of variants, is this selection in a Darwinian sense? Chapter 4 will attempt to give some answer to this question. 



\section{Chapter 4}

\section{Linguistic variants in cultural-evolutionary models}

\subsection{Language as an adaptive system}

Chapter 2 was about the mechanisms that support cooperation. It was argued that such mechanisms are supported by markers of identity and social affiliation, which help create a safe environment for cooperative exchange. The implication is that human beings are biased, as a result of biological evolution, to use such markers to guide their social behaviour. It may also be that human beings have evolved a bias towards relying on linguistic markers in particular, though this cannot be assumed to be the case - such a specific bias may be inherited culturally, not biologically (Section 10.1 will discuss this further). As was argued in Section 2.4.2, language is an inherently good source of reliable markers. It is worth noting that this is to a great extent the result of language being culturally transmitted: members of the same group talk in a similar way because they acquire linguistic variants from other members. This thesis is about modelling that process experimentally, and Chapters 5 to 9 will describe the model. Chapter 3 discussed the theoretical background from a relatively traditional historical-linguistic and sociolinguistic standpoint. The present chapter will also be concerned with a theoretical question, and one that has demanded attention ever since the word "selection" was introduced in Section 2.4.3; this word implies an evolutionary process - one, moreover, that is relevant to explaining the nature of language. This is not, however, quite the same as the process that made our brains language-ready. 
Indeed, the science of evolutionary linguistics has two foci. The first concerns the processes of biological evolution that led to human beings becoming a linguistic species, and the influence of evolved systems on the structure of modern language (e.g. Pinker and Bloom 1990; Deacon 1997; Hauser et al. 2002). The second treats language itself as a "complex adaptive system" (Gell-Mann 1992; Morowitz and Singer 1994; Steels 2000). The two approaches are not in the least contradictorythough there is certainly room for debate regarding their relative importance as explanations for particular features of language - and they cannot be treated as entirely separate. The constraints and biases to which language adapts are to a great extent the product of human biological evolution. Moreover, it is far from clear that the route that took us from our non-linguistic ancestors to our current linguistic state can be understood entirely through biological evolution; cultural evolution may well play a vital explanatory role not only in how and why language changes, but also in how human beings came to have such a thing as language, at least in the form that we do (Brighton, Kirby and Smith 2005; Kirby et al. 2007). The distinction implies a shift of perspective: on the one hand, human beings adapt to their environment and language is shaped to their needs; on the other, language itself adapts to the environment of human brains and social structures. Indeed, some cultural-evolutionary accounts (e.g. Croft 2000) make relatively little reference to human beings as active agents. Others are more careful to keep speakers in the picture; Keller (1994), for example, views language change as a "phenomenon of the third kind" - a large-scale unintended consequence of small-scale human intentions. Similarly, some make the link between cultural and genetic evolution very explicit, and argue that they are two examples of the same process (e.g. Lass 1997; Croft 2000; Ritt 2004; Mesoudi et al. 2004), while others are rather wary of the notion of uniting cultural and biological evolution as a single process. ${ }^{1}$

This is not only a concern of modern evolutionary linguists. The divide between those who focus on human evolution and those who focus on language evolution has its roots in a rather older divide in historical linguistics, between those who

\footnotetext{
${ }^{1}$ See, for example: Sperber (2000); Brighton, Smith and Kirby (2005); Andersen (2006); Ingold (2007). Barkow (2006) and O'Brien (2006) make reference to a very broad hostility in the social sciences generally towards perceived incursions from biology and other sciences, although this certainly does not lie at the root of many criticisms of the "same process" view.
} 
seek to explain change with reference to speakers and those who seek "some of the ultimate explanations for change in languages as systems" (Milroy and Milroy 1985: 345). Indeed, the view that language behaves rather like a biological entity is usually dated to at least the nineteenth century, and William Jones's recognition of the relatedness of certain languages that would come to be known as IndoEuropean - and the consequent hypothesis of their common origin - dates to the eighteenth. There are even examples of similar conclusions as early as the sixteenth (Lass 1997: 108). The direction of influence tended at this point in history to be from linguistics to biology, rather than the other way round; recognition of the organisation of languages into family trees, and the acceptance of language change and divergence, upon which this depends, was famously commented on by Darwin (1879/2004: 465f). That a coherent theory of linguistic common origins should have preceded the biological one is presumably due to the rate of change and divergence in language being so much greater than in biology, making such conclusions more readily apparent. There has long been influence in the other direction, however, and it is thus interesting that, by the twentieth century, the application of biological metaphors to language had nonetheless become "One of the most controversial issues in current historical linguistics (if not all linguistics)"; evolution had, indeed, become "a 'dirty word' in modern linguistic theory" (McMahon 1994: 314). Fitch (2007: 665) attributes this, at least partly, to the quasi-mystical ideas entertained by some nineteenth-century linguistics, like Jacob Grimm's Sprachgeist. McMahon (1994) attributes it instead to the careless borrowing of concepts from other fields by individuals who did not fully understand what they were borrowing (although the two phenomena may not be entirely distinct).

Andersen (2006: 60), on the other hand, argues that the adoption of biological metaphors in linguistics is simply part of a series of attempts by linguists "to legitimize their endeavors by borrowing concepts, principles, or methods from other sciences, natural or formal." He goes on to argue that:

Against such attempts to make linguistics 'more scientific' by borrowing from the 'real' sciences, it should be emphasized that there is only one way the practitioners of a discipline can make it truly scientific, namely, by developing a conceptual framework and methods of investigation that are appropriate to their object of inquiry. This is, of course, precisely 
what scholars in the natural and formal sciences have done and continue to do. (ibid.)

In his view, historical linguists have not in the main failed in this endeavour, and what metaphors they have adopted from the natural sciences "have generally been understood as the metaphors they are" (ibid.). He considers that modern evolutionary theory, on the other hand, is a poor fit to language change, and that many of its proponents have lost sight of the metaphorical nature of its application. In this last point, he would find some kind of agreement among these proponents: several cultural-evolutionary theorists (e.g. Lass 1997; Croft 2000; Ritt 2004; Mesoudi et al. 2006) explicitly reject an understanding of evolutionary theory applied to culture as "metaphorical". In their view, researchers of cultural and biological evolution are both studying the same fundamental process. Clearly there is some question as to the level of detail at which this is true. At the finest-grained level, it is trivially untrue (cf. Lass 1990: 96; Ritt 2004: 89-91) - languages are not made of DNA, for example - while at the most coarse-grained level, it is trivially true: both are complex systems that change. The question is whether there is a level of detail between these extremes where it is interestingly true that the mechanisms are the same, where recognition of the commonalities makes a useful contribution to our greater understanding. As Jäger (2010: 193) puts it, "The proof of the pudding is in the eating." He can be said to fall in the category of those who would treat language change and biological evolution as examples of the same process, but at a rather more abstract level than, say, Croft (2000) and Ritt (2004). He promotes an analysis based on George Price's "General Theory of Selection" (Price 1970, 1972, 1995), by which the same simple equation - the Price equation - can be used to model processes as diverse as biological evolution, trial-and-error learning, chemical recrystallisation, political change, and the selection of apples in the marketplace (Price 1995; Jäger 2008).

At the other extreme from these theorists are those, like Andersen, who view evolutionary theory as actually unhelpful to our understanding of language change. Somewhere between these poles are those who consider it a useful metaphor or analogy, in one way or another (e.g. Whinnom 1971; Mufwene 2001; see also Croft 2000: 10-13, for further discussion of this three-way distinction). 
There is a great deal that could be said about models of cultural evolution, and there is not space here to say more than a small amount of it. Chapter 3 talked of the innovation and propagation of linguistic variants, and their differential selection based on social factors, and Chapters 5 to 9 will present an analysis of experimental data based on such variants. The rest of the current chapter, therefore, will concentrate on evolutionary models of variant propagation. The question, which has been a significant focus of recent debate (Jäger 2010: 193), is whether such variants can be treated as "replicators" in the sense Dawkins (1989) uses the term. Is language, to use another of his terms, memetic?

\subsection{Memetic models of language}

A meme is a cultural replicator that can be the basis of evolutionary processes:

Examples of memes are tunes, ideas, catch-phrases, clothes fashions, ways of making pots or of building arches. Just as genes propagate themselves in the gene pool by leaping from body to body via sperms or eggs, so memes propagate themselves in the meme pool by leaping from brain to brain via a process which, in the broad sense, can be called imitation. (ibid.: 192)

For the idea of memes to be able to offer anything interesting to the study of culture, it is important that they be replicators in an evolutionary sense; as Sperber (2000: 163) notes, the Oxford English Dictionary's definition of a meme as "an element of culture that may be considered to be passed on by non-genetic means" divests the idea of any usefulness. Indeed, the term "replicator", as Dawkins (1982/1999: 83) defines it, is extremely broad: "anything in the universe of which copies are made. Examples are a DNA molecule, and a sheet of paper that is xeroxed." Only a certain kind of replicator can be made the focus of evolution, however: "active germ-line replicators" (84) - that is, replicators that have some influence over their probability of being copied (are active) and are potentially the ancestor of an indefinitely long chain of descendants (a germ line). Only these can be the focus of natural selection and have long-term evolutionary impact (ibid.). The qualities need for such a replicator to be successful are longevity, fecundity and fidelity. It seems clear that such replicators could exist in a form other than DNA, 
and even Sperber (2000: 163) — who is deeply critical of meme theory —acknowledges that "there are clear cases of actual memes", giving chain letters as an example.

Dawkins introduced the idea primarily as a thought experiment, but it has found enthusiastic support among such figures as Daniel Dennett (e.g. 1990; 1993; 1996) and Susan Blackmore (e.g. 2000; 2003). Others (like Sperber) remain more critical. It is intriguing, however, that it has not found greater support in linguistics; linguists are very used to breaking language up into discrete units, and there is no doubt, first that these units can be copied faithfully, and second that small modifications over time lead to large changes. The fact that the idea has not stimulated greater interest in linguistics may partly reflect a general opposition in the social sciences towards perceived incursions from biology (cf. Barkow 2006; O'Brien 2006; see also page 53). Ritt (2004: 119f), one linguist who is attracted to the idea, attributes it more positively to the "very long tradition of historical language studies as an academic discipline, and the progress they have made without employing an evolutionary paradigm" (cf. Andersen 2006). His view, however, is that an evolutionary paradigm has something important to offer the study of language change.

\subsubsection{Ritt and neural assemblies}

For Ritt, evolution is not an analogy in language change; language change is an evolutionary process, and a memetic one at that. Based on Dawkins (1989, 1982/1999) he gives four criteria for identifying replicators $(122-3)$ :

1. Minimal stability, or longevity.

2. Minimal copying fidelity. ${ }^{2}$

3. Minimal fecundity.

4. An effect on its own chance of being replicated or copied well.

This leads him to his own definition of a meme:

A 'meme' represents an assembly of nodes in a network of neurally implemented constituents, which has (a) a definite internal structure, (b) a definable position within a larger network configuration, (c) qualifies as a replicator in Dawkins' sense. (Ritt 2004: 169)

\footnotetext{
${ }^{2}$ For evolution to occur, of course, there is also a maximum level of permissible copying fidelity; mutation must be possible (Ritt 2004: 123).
} 
In language the neural structure of a phoneme would be a prototypical example, and he gives others. There is a problem with this, however, and it lies in the difference between how genes replicate and how memes replicate . Gene replication is a far more direct process; unless there is a fault in copying, gene replication leads to a new copy that is identical to the original, and which codes for proteins in the same way as the original. In language (or culture generally), however, a particular neural pattern in one brain is not directly copied into another brain; instead, it is inferred and reconstructed. Importantly, it is not obviously the case that the reconstructed neural pattern must be identical to the original; it has no a priori need to be, provided both produce the same output. Ritt is aware of this problem and considers it a serious one (2004: 90, 104-8 etc.). Yet he does not give a very clear answer to it, partly because we simply do not know enough about the neural underpinnings of stored linguistic information. The problem of differences between genetic and memetic replication is a challenge for any memetic model (cf. Sperber 2000; Brighton, Smith and Kirby 2005: 221-2), but Ritt's desire to locate the replicating unit in neural assemblies is particularly troublesome in this respect.

The problem is not even quite as empirical as it might appear on first sight. The important question is not whether or not linguistic units are in fact consistently represented by identical neural configurations; it is more useful to ask whether it is necessary that they be so represented. Ritt argues convincingly that language evolution can be understood in terms of discrete units; he argues, indeed, that to linguists this is "almost old hat" (203) — traditional linguistic theory has long divided language up into such units as phonemes, morphemes, lexemes, and so on. This would seem to fulfil his first criterion for identifying replicators (minimal stability). ${ }^{3}$ Ritt further argues convincingly that these units are copied with a very high degree of fidelity. This again, is not a new claim in linguistics: language learning is clearly a faithful enough process that children are able to talk easily with their grandparents (and easily understand the recorded output of several generations beyond that), in all but exceptional circumstances; and it is far from clear to what extent those differences that exist between generations can be explained by copying error. As Ritt notes, many linguistic units remain unchanged over thousands of

\footnotetext{
${ }^{3}$ Whether or not these categories are in fact suitable will be discussed in more detail in Section 4.2.3.
} 
years (133). Linguistic transmission thus seems very clearly to be a faithful enough process for the second criterion (minimal fidelity) to be fulfilled. The third criterion (minimal fecundity) is also no problem; no one denies that linguistic variants are transmitted between brains. The fourth criterion (that the replicators have some effect on their own likelihood of being transmitted) is also fulfilled; phonemes that are not sufficiently distinct from others in the same system, for example, are likely to be harder to copy than others.

Ritt, therefore, has already made his key arguments for the existence of some kind of linguistic unit that can be the focus of evolutionary processes, and none of this is dependent on the unit being instantiated on the level of neural patterns. The problem described above, of whether or not the copying process produces identical neural configurations embodying these units, is a question about the nature, or composition, of the units, not about their existence. If the units are thus already accepted to exist, then the question is this: is it in principle possible that such linguistic units as phonemes could be copied as faithfully as they are, yet have different underlying neural representations? If it is impossible, then their very existence shows that the units must therefore exist at the neural level, and that it must be these neural configurations that are copied faithfully. If, on the other hand, we accept the possibility that they could be underpinned by different neural representations, yet have the same surface features as consistently as they obviously do, then the copying fidelity cannot be a feature of the neural configuration, but must reside at a different level. In which case the replicator itself must exist at a different level. Should it then turn out, in fact, that the units are underpinned by identical neural patterns, then this must be coincidental.

\subsubsection{Croft and utterances}

If the linguistic replicators do exist above the level of neural patterns, at what level is this? Presumably it must either be at some relatively abstract level, or at the acoustic level. This latter possibility recalls Croft's (2000) view that the linguistic replicator is the utterance, or, more specifically:

embodied linguistic structures, anything from a phoneme to a morpheme to a word to a syntactic construction, and also their conventional 
semantic/discourse-functional (informational-structural) values ... The linguistic structures as embodied in utterances are not beads on a string either. They exist in nested systems of more inclusive units (28)

That Croft should choose the utterance as the unit of selection seems to arise from his commitment to treating language in terms of "real, existing entities" (2). The same commitment plays a role in Ritt's choice of neurally instantiated units, and it is understandable. DNA is tangible; it can be looked at under a microscope. If linguistic replicators can also be shown to exist in some physical form, then this is reassuring. It is, however, rather odd that Croft should choose the utterance as the home of his replicators rather than the brain (although the degree to which he keeps this distinction clear is highly questionable; see below). He does not raise the problem noted above of how neural patterns might be replicated, and he acknowledges the attractiveness of the obvious analogy between the grammar as the genotype and the utterance as phenotype - to which Ritt explicitly subscribes. As Croft points out, while in biology the replicator (the genotype) produces the interactor (the phenotype), the situation appears to be reversed in his conception of language evolution (39), where the utterance-level units are the replicators, and the grammar the interactor. The key to his choice seems to be that:

the replicator ... should not be identified with the structure as an abstract essence (type). The formal structure of e.g. the passive construction or its semantic/discourse function, or the phonetic expression of a phoneme can change in replication. (28)

Ritt (2004: 158) considers that Croft's utterance-based theory "is flawed in a fundamental way." He takes issue with Croft's definition of an utterance as:

an actually occurring piece of language, completely specified at all levels of structure, including its full contextual meaning on the particular occasion of use (i.e. speaker's meaning). (Croft 2000: 244)

Ritt is right to see this as a serious problem. Croft seems to want to have his cake and eat it: his conception of an utterance includes not just acoustic (or, indeed, signed or graphic) patterns, but mental states as well. Such units cannot be the "spatiotemporally bounded individuals" (Croft 2000: 24) that he wants them to be. It is easy to see why he would want his units to consist of more than acoustic or 
graphical patterns; if he does away with the mental components, then, as Ritt (2004: 159) points out, he has trouble keeping such forms as "son" and "sun" distinct, or in establishing "that two allophonic realisations of any morpheme actually represent variants of a single type" (ibid.). However, if linguistic replicators are to exist on the level of the utterance, and not mentally, then all they can be is strings of sounds - waveforms. The contextual meaning that Croft refers to must form part, not of the units themselves, but of the framework for their replication. If Croft is serious about having meaning form part of the replicator, then that replicator must exist in the minds of the speaker and hearer, and the waveform (together with the context that allows inference of meaning) is in fact the means by which replication occurs (i.e. the interactor), not the replicator itself. It may be that this is what Croft intends in his definition - the memory of an utterance, in other words, rather than the utterance itself - in which case, his units exist, like Ritt's, in people's heads. If so, where precisely should these mental replicators reside? As discussed in Section 4.2.1, Ritt's view that they must be instantiated as patterns of neurons, while plausible, is problematic for reasons already given, and it would be better to treat his replicators as informational units, perhaps instantiated on a more abstract level, but still a mental one. If Croft is serious in what he wants "linguemes" to consist of, then they too seem best understood as existing at this level, rather (in this case) than in "actually occurring ... language", as he claims to want.

The key questions are as follows: first, do linguistic units in fact exist that can be the focus of evolutionary processes? Second, if they do, do they exist outside the heads of speakers or inside them? If the former, then they can consist only of waveforms. If the latter, then they can consist of more than this, but the question remains open as to what level they exist on. Ritt sees them as existing as neural patterns, but if we cannot be certain that such neural patterns themselves are reliably replicated, then it is in fact the information they instantiate that is the replicator, since it is here that their reliability must reside. If we cannot accept this, then the units must exist as neural patterns, and we are committed to either assuming that the neural patterns can be replicated faithfully or to rejecting such mental units altogether. The next section will discuss whether linguistic replicators 
can be said to exist at all, and whether the kinds of linguistic unit that Croft and Ritt identify as candidates are really suitable.

\subsubsection{Candidates for linguistic replicators}

Both Croft and Ritt agree in identifying traditional linguistic units, like phonemes, morphemes, rules and syntactic constructions ${ }^{4}$ as examples of linguistic replicators (see e.g. Croft 2000: 33-7; Ritt 2004: 169-86). This is perhaps not surprising; it is hard to see what other units would do-Ritt (2004: 133-5) argues convincingly that smaller entities, such as distinctive features, are not sufficiently cultural (that is, they are too grounded in basic human abilities) to count. He argues further that sequences of phonemes below the morpheme level are not sufficiently stable; that is, we should not expect them to be replicated or stored distinctly from higher-level units. This argument seems to hold as far as most such sequences are concerned, but does not succeed in excluding phonaesthemes. Of course, there is no principled reason why units between the phoneme- and the morpheme-level should not also be considered to be linguistic replicators. Nevertheless, phonaesthemes seem problematic; on the one hand, this most likely simply reflects their uncertain status in mainstream linguistics (Bergen 2004); on the other hand, even if their existence is taken for granted, there is a problem with treating them as replicators - to the extent that they are units with any psychological reality, they are likely to be schematic units inferred by the speaker from statistical tendencies over distributions of phoneme sequences.

This fact raises a rather bigger problem: to what extent is this different from other, more canonical, linguistic units? Are phonemes, for example, any safer than phonaesthemes? There is much evidence now that speakers retain a great deal of phonetic tokens in memory, and that phonemes are categories with considerable internal structure - schematic units based on clusters in phonetic distributions. ${ }^{5}$ As Bybee (2007: 205) puts it:

\footnotetext{
${ }^{4}$ As the inventor of radical construction grammar, Croft understandably puts more emphasis on constructions than on rules.

${ }^{5}$ Miller (1994) is a relatively early review of evidence; Pierrehumbert (2001) provides a more recent summary. See also Wedel (2006) for a summary very relevant to the current question.
} 
Phonemes ... are not units of lexical representation. Instead, phonemes are abstract patterns that emerge in the phonological organization of the lexicon.

Exemplar theory has also convincingly been applied to other levels of linguistic structure (Bybee 2006; Bod and Cochran 2007). This model leaves a level of uncertainty as to the status of the linguistic "units" as potential replicators. If it is correct, then linguistic replication of phonemes and rules is not a matter of a cognitive unit being copied, via an acoustic channel, from one brain to another. Instead, a speaker uses a mental category, representing a structured collection of memorised tokens, to produce an acoustic token, and this token is turned into a mental representation by a listener, which forms part of a structured collection of mental tokens in the listener's brain, influencing the phonetic forms that a speaker is likely to produce in future. There are three types of unit here: an acoustic token, a mental token, and a mental category (the second of which has something in common with Ritt's replicators). Indeed, there are at least two kinds of category: since the mental tokens, which contain the phonetic detail, are supposed to be stored in the lexicon (and lexical items are thus considered to be rather more phonetically specified than previously thought), the basic category derived from them is on the lexical level, and any sound-unit level of category must be derived from this level. Much the same, within generalised exemplar theory, is true of morphosyntactic units. Where does that leave our replicators? This, perhaps, is why Croft, a construction-grammarian, seeks refuge in the utterance. Within the confines of this theory, it seems that the replicator, if there is one, would have to be the token, not the category. It seems reasonable to say that phonetic tokens are copied. If categories are derived from multiple tokens gathered from multiple interactions, on the other hand, it is hard to see that there is anything like direct copying going on. In any case, Croft's lingueme, as discussed in Section 4.2.2, does not seem to keep this distinction as clear as one might expect.

\subsubsection{Background conditions}

Even ignoring exemplar theory, the inferential means by which linguistic variants are transmitted may be a problem. Sperber (2000), for example, argues forcefully that 
the means by which cultural units are transmitted changes the game. Linguistic replicators are not imitated; instead, the instructions for producing linguistic forms are inferred through observation of another speaker's output (Brighton, Smith and Kirby 2005 also stress the importance of this). More specifically, he argues that this inference depends on information already present in the system:

Thus, the similarities between the grammar and lexicons internalized by different members of the same linguistic community owe little to copying and a lot to pre-existing linguistic, communicative, and conceptual evolved dispositions ... For memetics to be a reasonable research programme, it should be the case that copying, and differential success in causing the multiplication of copies, overwhelmingly plays the major role in shaping ... the contents of culture. Evolved domain-specific psychological dispositions ... should be at most a relatively minor factor that could be considered part of background conditions. (172)

Put this way, the argument is only partly convincing. Pre-existing evolved dispositions of this sort may be a good explanation for certain universal features of human language (though see, for example: Keller 1994; Kirby et al. 2004; Brighton, Kirby and Smith 2005), but they hardly explain the similarities to be found between members of a linguistic community, as compared with a different linguistic community. The fact that people from London are more like other Londoners than people from Washington DC in their propensity to pronounce "butter" with a glottal stop (or, for that matter, the fact that people from Caernarfon are now more likely to speak Welsh than people from Carlisle) has nothing to do with pre-existing evolved dispositions, except inasmuch as there is a disposition to acquire one's grammar and lexicon from other members of one's own community. Moreover, while there are of course pre-existing cognitive and physical constraints on linguistic replication, there are physical and chemical constraints on genetic replication too. It is hard to see how these should not be considered background conditions to the propagation of linguistic variants. Nevertheless, the key question that Sperber raises is a significant one: are the inferential mechanisms by which a linguistic unit is replicated sufficiently similar to the mechanisms by which DNA is replicated for both to be seen as the same kind of replicator? Again, it must be stressed that much depends on the level of detail. On a certain level of detail, the two processes are obviously 
different; on a very coarse-grained level, they are clearly similar. Sperber marks out a middle ground with three minimal conditions for "true replication". Specifically:

For $\mathrm{B}$ to be a replication of $\mathrm{A}$,

1. B must be caused by A (together with background conditions).

2. B must be similar in relevant respects to $A$, and

3. The process that generates $\mathrm{B}$ must obtain the information that makes B [relevantly] similar to A from A.

(Sperber 2000: 169)

It is not too hard to find a linguistic example that seems to work within this model. Consider the following situation: an English speaker (A) produces the phonetic form [mi?] which is heard by another English speaker (B) and internalised by that speaker. Speaker A must have mental instructions to produce the form [mi?]; this is a competence state (cf. Ritt 2004). If we take a relatively exemplar-theoretic approach and assume that phonemes are not monolithic units, this competence state must either be a mental token previously added to the lexicon (and categorised) after being acquired from another speaker, or a mental token derived in some way -involving phonemic categorisation - from other tokens in the lexicon (this token might have been created at a previous point in the past, or might be created at the moment of speech).

In either case, speaker A has a mental linguistic token that has a causal relationship with the acoustic token produced; this mental token can be termed $\mathrm{Am}_{\mathrm{m}}$. A's acoustic token, $\mathrm{A}_{\mathrm{a}}$, is heard by $\mathrm{B}$ and transformed into a mental token $\mathrm{Bm}$. It seems clear that there is a strong causal relationship between $\mathrm{A}_{\mathrm{m}}$ and $\mathrm{A}_{\mathrm{a}}$, and that there is also a strong causal relationship between $\mathrm{A}_{\mathrm{a}}$ and $\mathrm{B}_{\mathrm{m}}$; the causal relationship between $\mathrm{Am}_{\mathrm{m}}$ and $\mathrm{B}_{\mathrm{m}}$ is more indirect simply because of the acoustic stage, but it is hard to feel that this is a significant barrier to saying that $\mathrm{Am}_{\mathrm{m}}$ causes $\mathrm{B}_{\mathrm{m}}$, together with background conditions - these being the cognitive and physiological mechanisms that allow A to turn $\mathrm{A}_{\mathrm{m}}$ into acoustic patterns and allow $\mathrm{B}$ to hear and interpret these patterns. Furthermore, inasmuch as $\mathrm{B}_{\mathrm{m}}$ leads $\mathrm{B}$ to reliably produce acoustic patterns relevantly similar to those that $A_{m}$ led A to produce, then Sperber's second criterion is also fulfilled. The third criterion is the most important, and Sperber also expresses it as, "B must inherit from A the properties that make it relevantly similar to A" (ibid.). This also seems to be fulfilled: the properties 
that make $\mathrm{B}_{\mathrm{m}}$ relevantly similar to $\mathrm{Am}$ are the particular acoustic patterns it leads a speaker to produce in a suitable context, and these must come from $\mathrm{Am}$.

The crux of the argument lies in the two words "relevant" and "background", and it is important to note that Sperber seems to be focusing primarily on the acquisition of a first language by an infant. Given that, his point is fair: in the first stages of language acquisition, those cognitive and physiological mechanisms described above as background conditions are in the process of being developed, and the development of these probably should not be considered a matter of replication. Sperber seems to be right about that. At least some of the competence states that exploit these mechanisms, on the other hand, seem very reasonable candidates for evolutionary replicators.

\subsubsection{Phonetic tokens as a special case}

Brighton, Smith and Kirby (2005) make a similar argument to Sperber's:

DNA persists by a process of direct copying governed by a selective mechanism that prunes lines of inheritance. Linguistic knowledge, on the other hand, must persist through a repeated cycle of production and induction. We can think of the task of the learner as akin to that of the reverse engineer, trying to figure out what the blueprints are for a device while only being able to look at its behaviour. In the system of biological evolution there is no such reverse engineer - the blueprints are passed on directly every generation. (221)

This is very close to Sperber's argument, cited on page 63, but it is less committed to the existence of evolved domain-specific mechanisms. Put simply, the copying of linguistic variants is rather less than direct. This points at a difference, noted before, between sound patterns and other linguistic structures. The model of transmission given in Section 4.2.3.1 is really about the transmission of phonetic variants. It does not work anywhere near so neatly for semantic and syntactic structures, or for the phonemic categories built from these phonetic tokens. ${ }^{6}$ It was noted in Section 4.2.2 that all that a spoken utterance consists of, apart from what is in the heads of the speakers in the interaction, is a waveform. This is a fundamentally

\footnotetext{
${ }^{6}$ Note that this is true even given a less exemplar-theoretic approach. Any theory has to have some way of building phonemes secondarily, through a process of categorisation, from phonetic tokens.
} 
important fact, which makes phonetic variants different from semantic and syntactic ones: phonetic variants physically exist outside speakers in a way that no other linguistic variant does. The meaning of an utterance and the morphosyntactic constructions that govern the arrangement of meaningful elements must both be inferred - reverse engineered - and they are inferred from the phonetic string once it has been internalised and become a mental token in the mind of the hearer.

This is not to say that the transmission of meaningless (i.e. phonetic) tokens does not also involve reverse engineering. It must do, since a waveform is not the same as the instructions for producing a waveform. And in infants who are learning to speak, or adults learning non-native sounds, this reverse engineering is not trivial. However, in the relatively narrow (yet overwhelmingly typical) case of adults hearing new strings that follow familiar phonotactic patterns, there is a good case to be made that this inference plays a relatively minor role- for its having been relegated, in other words, to a background condition. Consider, for example, an adult native English speaker (A) teaching another adult native English speaker (B) the novel word $[\mathrm{gl} \Lambda \mathrm{bl}]$. Assuming a relatively noise-free channel, we would expect $\mathrm{B}$ to be able to repeat the word back with little trouble (by contrast, say, with a resolutely foreign name like $[\underset{\mathrm{T}}{\mathrm{i}}]$ ] $]$. It seems reasonable to talk of a relatively straightforward transmission of a competence state $\mathrm{A}_{\mathrm{m}}$ - via an acoustic pattern $\mathrm{A}_{\mathrm{a}}$ - to competence state $\mathrm{B}_{\mathrm{m}}$. Of course there are entire cognitive frameworks set up, conditions that have to be in place, to make this transmission possible; of course this is not quite direct copying; but there are conditions that have to be in place for DNA replication too. This by itself cannot be a cogent objection.

There is, in fact, a way of imagining the replication of sound-patterns that makes it look somewhat similar to the replication of DNA strands in an interesting way. Consider how DNA replication takes place: a double helix splits in half and each half is used, reliably, to reconstruct a new copy of the missing other half. This, of course, relies on the appropriate mechanisms being in place for such reconstruction to be possible. A phonetic token, similarly, can be seen as consisting of two halves - a competence half and an acoustic half. ${ }^{7}$ The reconstructibility of one from the other,

\footnotetext{
${ }^{7}$ Granted, there is a difference here. Both halves of a DNA strand are of the same kind, while the two halves of a phonetic string given here are not. This must simply be accepted; to insist that biological and linguistic replicators must be identical in every way helps no one. The point is
} 
given the appropriate mechanisms, seems reliable in a way that the reconstructibility of a strand of double helix is reliable.

So can phonetic tokens act as the linguistic replicators we want? Certainly they seem more suitable than other candidates. A few points should be made clear. First, these tokens must be phonetic strings, not individual phonemes. Phonological knowledge must already be in place - must already be part of the background conditions - for the above to work as described. Second, it must be mental tokens, not acoustic tokens, that are the replicators. There are numerous differences between any two acoustic tokens, even produced by the same speaker, that are not linguistically relevant. Furthermore, as Ritt (2004: 159) pointed out, there is no way to distinguish "sun" from "son" acoustically, except by inference from context. Mentally, such tokens can be attached more clearly to semantic information, though, as implied, this meaning does not form part of the replicator. Since de Saussure (1916/1995), a linguistic sign has been seen as consisting of two parts, the signifier and the signified. The phonetic replicators described above are signifiers in this sense; they are not full signs (cf. Ritt 2004, 233, who also decouples the two halves of the sign, and treats linguistic signs as "secondary replicator alliances").

Where, then, does this leave meaning? For language to work, the phonetic tokens described above must be paired with meanings. The way in which meanings are transmitted, however, is different from the way in which phonetic strings are transmitted; the process is much more clearly, and importantly, inferential. While the transmission of phonetic tokens (at least between adult native speakers of the same language) may be reliable enough, owing to the nature of the transmission channel - which can contain only phonetic strings - for these tokens to be good candidates for evolutionary replicators, the transmission of meanings is different and relies much more on relatively uncertain reverse engineering. Indeed, the reliable inference of meaning of a particular word or construction can easily depend on the hearer being exposed several times to a variant. There is a similar problem with syntactic constructions. In traditional linguistics, syntax has been treated rather

that, in order to replicate, DNA strands split and create two halves from what was a whole. This is the means of its replication. The means by which a mentally stored phonetic string replicates is by producing something new-an acoustic token. 
differently from morphology, with the emphasis being on rules. In usage-based models of language, such as Cognitive grammar and Construction grammar, however, syntactic constructions are treated more like morphemes: phonetic strings with attached meanings, or signifer-signified pairings. From this point of view, should the phonetic half of these be treated differently from the word-level phonetic tokens described above? There is a difference. Syntactic constructions are templates, derived, like meaning and phonological categories, by a process of inference from utterances and with regard to the context in which they occur, and this is where a crucial difference lies: phonetic tokens are independent of the discourse context in a way that other linguistic variants are not.

\subsection{Conclusion}

It has been suggested in this chapter that there are, in general, problems with treating linguistic variants as Darwinian replicators, but that word-level phonetic tokens stand out as plausible candidates for such replicators, owing to the nature of linguistic transmission. Even these, however, only qualify in a relatively constrained set of circumstances: the transmission of variants among adult speakers with a high level of competence in the language in question (and given a relatively exemplar-theoretic account of transmission); only here is there sufficient automaticity in the replication for the inferential capacities involved to reliably be relegated to background conditions. Constrained though it is, however, this context cannot be considered rare. It is reasonable to expect that most linguistic interactions in the world are between adults competent in the relevant language.

It should be noted that there remain important differences between language evolution and biological evolution, even if phonetic tokens are treated as evolutionary replicators. The rate of change in language, for example, is clearly greater than in biology. Brighton, Smith and Kirby (2005: 221-2) make a further point:

there may be times where competition leads to the induction of totally new rules in one step. Linguistic paradigms can change through a process of "reanalysis", the output of a number of different rules can lead to the origins of a new rule that subsumes them all-indeed, this process is fundamental to the evolution of increasingly general regularity in the iterated learning models we have described. 
This is particularly true of grammatical structures in language; it is here that this sort of reanalysis occurs, rather than directly on the phonetic tokens paired with particular meanings - and this is a further reason to treat these as different. Nevertheless, this does not mean that competition between phonetic forms too cannot lead to the production of new variants, and this is clearly different from biological evolution.

Much depends, however - as stated elsewhere in this chapter - on the level of detail, and at a certain level of detail, it is not even clear that the difference between phonetic and other variants matters. How important is it that there is a difference in the directness of transmission, if there is transmission and it is faithful? On another level of detail, we can, as Wedel (2006) does, model category shift in an individual speaker's brain as an evolutionary process. It seems there is something to be lost here in the search for the "correct" linguistic replicators to match up with genes. It does not seem true that language change can be reduced to competition between exemplars in the head of a single speaker (and Wedel 2006 does not think it can), or to differential propagation of variants between speakers. ${ }^{8}$ It seems clear that selection can operate in both cases; it also seems clear that mutation can occur in more than one place. Assimilation, for instance, must originate at least partly as a result of gestural overlap in the mouth of a speaker (though Ohala 1990 argues for a significant role for the hearer). Dissimilation, on the other hand, is almost certainly better explained as hearer-based. Similarly, while grammaticalisation may originate in an intentional metaphorical extension on the part of the speaker, in some cases it will be entirely driven by processes taking place in the minds of hearers (Hoefler and Smith 2009).

This should not discourage cultural-evolutionary approaches to language change. As Boyd and Richerson (2000: 158) put it:

If the application of Darwinian thinking to understanding cultural change depended on the existence of replicators, we would be in trouble. Fortunately, culture need not be closely analogous to genes ... All that is really required is that culture constitutes a system maintaining heritable variation.

\footnotetext{
${ }^{8}$ As argued above, if we want to take a variant-eye's view to the latter process, we need to take on board the distinction between phonetic tokens and meaningful variants.
} 
To ignore the similarities between language and biological organisms would be to ignore important parallels that have been beckoning to scientists for at least two centuries. The danger is in attempting to shoehorn language into a genetic boot that does not fit it, particularly when there exist generalised frameworks such as the Price Equation, which allow questions of what the "right" replicators in language are to be sidestepped (Jäger 2010: 196).

Such frameworks, in making clear the commonalities between language and other complex adaptive systems, are a very useful addition to the historical linguist's toolkit. The next chapter will discuss the importance and role of experimental methodologies in this kit, and it will present the first of a series of experimental simulations designed to get at the question of the role of social marking in language divergence. 


\section{Chapter 5}

\section{Testing the hypothesis: a first experiment}

This chapter, along with the following four chapters, will present a series of experiments designed to investigate the roles of a pressure for social marking and frequency of interaction in language divergence. Section 5.1 will briefly discuss methodology in historical linguistics and will introduce the method - experimental simulationemployed in this thesis. Section 5.2 will describe how the first experiment worked, and Section 5.4 will present the results. Chapters 6 to 8 will describe experiments that follow on from the one described in the current chapter and attempt to pick out more finely the factors involved in new-dialect formation. Chapter 9 will then summarise the results and discuss various issues relating to them. Chapter 10 will discuss how the findings might fit into an account of the evolution of language, and Chapter 11 will present overall conclusions.

\subsection{Methods in historical linguistics}

Historical linguistics is not a field commonly associated with experiments. This is perhaps not surprising, given the time periods normally involved in language change. ${ }^{1}$ To be sure, any item of real-life linguistic data, whether already recorded in some medium or gathered fresh by the researcher, gives only a window onto

\footnotetext{
${ }^{1}$ This chapter - and, indeed, this thesis - will treat historical linguistics as the study of language change. Although this is broadly true, not everyone who calls themselves a historical linguist is interested in language change. Historical sociolinguists, for example, tend to be more interested in particular synchronic time slices (see Milroy and Milroy 1985, 344-5, for further discussion of such matters). A distinction is sometimes drawn between historical linguists, as scholars interested mainly in change, and linguistic historians, who are more interested in describing the linguistic situation at particular periods, or the history of a particular language. In practice, the first term is often used for both.
} 
a synchronic time-slice of language; and at this level of data collection, historical linguistics, sociolinguistics, and dialectology are hard to distinguish. If such data are to shed much light on anything, there has to be an element of comparison, and to the extent that variationist sociolinguistics and historical linguistics can be said to differ in method, it is mainly in the role of time in the comparison (Milroy and Milroy 1985: 344; see also Section 3.1.2 of this thesis). The items to be compared, for example, may come from different points in the same speaker's lifetime (e.g. Harrington 2006), or from different speakers separated by time, or, as in apparenttime studies, by age. Similarly, reconstruction relies on the comparison of variants of one variable at a particular time.

This, at least, is the traditional approach, and it has been augmented in the last few decades by the use of advanced statistics and phylogenetic methods, often borrowed from other disciplines, in particular biology (see e.g. McMahon and McMahon 2005). These methods have made a significant contribution, but their contribution is in the manipulation of data already gathered, rather than in expanding the means by which it can be gathered in the first place. Most historical-linguistic data is still gathered in the wild. There are, granted, several exceptions: the results of many psycholinguistic experiments, for example, shed important light on historical linguistic phenomena. Section 3.3.1.2 discussed accommodation and interactive alignment in dialogue. Much research has shown how this mechanistic process occurs more or less automatically in linguistic interactions, and contributes to communication. It is also generally assumed to play an important role in the propagation of linguistic variants, and thus in accent change and dialect formation (see e.g. Pardo 2006, and also Chapter 3 of this thesis). There are also the kinds of experiment commonly carried out in sociolinguistics, such as matched-guise tests, which provide information on language attitudes. Laboratory phonology, moreover, allows the collection of more detailed phonological data (or, more accurately, phonetic data that allows the drawing of phonological conclusions).

In the examples given so far, the diachronic dimension is clearly present only in the experiments on interactive alignment; in these, there is a clear change in language use over the course of the experiment. Assuming that frequency affects storage and categorisation, this can be expected to lead to, or affect the course of, 
grammatical change (Bod et al. 2003; Bybee 2006, 2007). In laboratory phonology and most sociolinguistic experiments, by contrast, the emphasis is on obtaining a clearer synchronic window. To model larger-scale, longer-term change, the most obvious recourse has been computational simulation. The advantages of computational simulations are obvious. Wild data are messy, and the different constraints and pressures operating on the individuals involved are not always clear, or even discoverable, to the researcher. In experiments, these can be controlled to an extent (at the expense of ecological realism), but not all unknown quantities can be eliminated. In the artificial environment of a computer simulation, on the other hand, the agents and the pressures acting on them are entirely under the programmer's control (at least within the constraints of the computational medium). For the historical sciences, computational simulations have an enormous further advantage: time can be contracted and thousands of generations modelled, many times over, in a matter of days. Such simulations have been widely used in evolutionary linguistics (for a review, see Cangelosi and Parisi 2002; Kirby 2002) —understandably, given the lack of available naturalistic data. They have not been neglected in historical linguistics, however (e.g. Nettle and Dunbar 1997; Nettle 1999; Livingstone and Fyfe 1999; Livingstone 2002; Oudeyer 2005; de Oliveira et al. 2008; Kirby to appear). Nettle and Dunbar (1997) present a model that is particularly relevant to the central question of this thesis. In this model, the existence of variability in language, ${ }^{2}$ coupled with a bias towards cooperating with those who speak in a similar way, leads both to a population that cannot be invaded by "CHEATs" and to a high level of (geographically structured) linguistic diversity. Nettle (1999: 37-59) describes a series of simulations that confirm these results, but suggest further that:

Geographical isolation and noise in learning are ... not sufficient to produce diversity in discrete variables unless the level of noise is set extremely high ... A very low level of inter-group contact, in the absence of social selection, destroys local diversity ... [S] ocial selection hugely increases both the amount of diversity which evolves and its stability against inter-group contact. (54)

\footnotetext{
${ }^{2}$ Although "language" in such simulations could be taken to stand for almost any sufficiently complex culturally acquired system.
} 
On the other hand, other computational simulations have cast doubt on the importance of social selection in explaining linguistic diversity. Livingstone and Fyfe (1999) and Livingstone (2002) describe simulations in which "child" agents learn language from "adult" agents; group boundaries are not defined, but individuals are more likely to interact the closer they are to each other spatially. Given random noise in the learning data, a high level of geographically structured linguistic diversity emerges, without the need for social selection.

The simplicity of computational agents and computational environments is a curse and a blessing. While it certainly makes both more tractable, much depends on the programmer's assumptions about the right biases to build into the model. The middle ground between simulations and studies of the real world, as implied above, is the experiment, and experiments vary considerably in their similarity to both. There is a kind of sociolinguistic study, for example, where participants are recorded talking in different conditions (e.g. chatting naturally and reading scripts) and the conditions compared. Here, the experimental manipulation is slight, and the difference from naturalistic data collection minimal. At the other end of the spectrum are studies that are best called experimental simulations. ${ }^{3}$ In embodied-agent simulations, for example, artificial agents (i.e. robots) are put in real environments (e.g. Vogt 2000; Quinn 2001). Alternatively, human agents can be put in highly simulated environments. This may involve complete immersion in a virtual reality (see Fox et al. 2009 for a review of this methodology in the social sciences), but any task, such as a game, intended to model some real-life situation achieves a similar effect. Studies of this sort have been employed with some success over the last decade in investigations of the origin and evolution of language (e.g. Galantucci 2005; Kirby et al. 2008; Scott-Phillips et al. 2009), but somewhat less so in research into change in modern language. The rest of this chapter will describe an experimental simulation, which was influenced in its design particularly by computational simulations described by Nettle and Dunbar (1997) and Nettle (1999) and by such experiments as Garrod and Doherty (1994) — which showed how communities can converge on a communicative strategy through paired

\footnotetext{
${ }^{3}$ This term has a long history in various fields; see e.g. Lindeman (1942); Mendlowitz and Leslie (1942); Waters and Glass (1970); Martindale (1973); Krackhardt and Stern (1988); Willis and Long (2007); Mesoudi and O'Brien (2008).
} 
interactions. It was carried out in November and December 2007 and designed to investigate the role of social marking in language divergence. ${ }^{4}$

\subsection{Description of experiment}

\subsubsection{Overview}

This experiment involved twenty participants, who were divided into five groups of four. Each of these five groups played a computer game, for which they were divided into two teams of two. The object of the game was for one's own team to score more points than the other team.

A game consisted of a series of fourteen timed rounds (see Figure 5.2), in each of which every player was paired pseudo-randomly (see Section 5.2.3) with one of the other players (their "partner" for that round). A round of the game involved using an instant-messenger-style program to negotiate to exchange resources (i.e. points, expressed as quantities of meat, grain, water, fruit, and fish). Players were not, however, allowed to use any real language, but had to type messages in an "alien language", which consisted of nineteen randomly generated words (see Section 5.2.6). After the negotiation stage of a round, players could choose to give away some of their resources to their conversation partner. The game was set up in such a way that it was advantageous to exchange resources with one's own teammate, but not to give anything away to one's opponents (see Section 5.2.5). As noted above, the four players of each game were divided into two teams of two, and players did not know for sure until the end of a round whether they were paired with their teammate or an opponent. Throughout the game all the players worked in separate booths and could not see each other. The only information they were given about the identity of their teammates was that they would be paired with

\footnotetext{
${ }^{4}$ The computer program that participants of this experiment used was written using a combination of HTML, PHP, and MySQL. I was aided in the programming by Justin Quillinan. While I designed the experiment, how the program should operate, and how the interface should look (which included writing the HTML code), he wrote the PHP and MySQL code that allowed participants on different computers to communicate with each other, and which stored the resulting data. The scripts written to analyse this data (which were all written using Python and R) were entirely my work. Details of the experiment described in the following sections, and the two experiments described in the following chapters, have been published in Roberts (2008) and Roberts (2010), and this thesis will draw on those publications, but will contain additional analysis and go into greater detail.
} 
them for the first two rounds of the game. No such information was provided for the other rounds. Only at the end of a round, after they had already chatted with the other person, and after they had already decided whether or not to give anything away to them, was it revealed who they had been paired with.

The uncertainty that this engendered put pressure on players to use variation in the alien language to mark identity, since this was their only means (aside from giving away resources) of interacting with anyone else in the game. At the start of the game, every player learnt the same alien language, so variation between players had to arise during the experiment, and could have only two sources: error and deliberate innovation. As well as the greater control afforded by this fact, there were two further advantages, from an experimental point of view, to using an artificial language. First, because the players were not using their own languages, but were using a language they had only learnt that day, the chance of error as a source of variation was increased. In other words, the mutation rate was raised, leading to considerably faster change than would normally be expected to occur (cf. Kirby et al. 2008). Second, as can be seen from the list in Section 5.2.6, the words available to players severely limited their opportunities for explicit strategising.

\subsubsection{A typical round}

In a typical round, players would usually begin by greeting each other. Typically, one would then state what resources they wanted, perhaps adding what they were prepared to give away, and asking what their partner wanted in return (e.g. "I need lots of meat. Do you have meat? I give you fruit."). The other would reply to this and the conversation would develop along these lines. Once this chatting stage had ended (it lasted 180 seconds), a player could choose to give away as many or as few resources as they liked (up to the amount they had available), and what a player did would depend on what was said during this exchange and on the player's estimate of the benefits of doing so. After this, they would be told the results of the exchange: what they had given away, what they had received, and whether or not they had been talking to a teammate. 


\subsubsection{Research questions}

Two main predictions were made:

1. That, in spite of the anonymity of the interaction, players would be able to identify their teammates using linguistic cues - the only means at their disposal.

2. That the use of the alien language as a source of group markers would lead the language, identical for all players at the beginning of the game, to begin to diverge into dialects.

There were also two secondary research questions: what strategies would the players use to win the game, and to what extent would success at recognising teammates and linguistic convergence between teammates correlate with score?

\subsubsection{Participants}

Twenty participants were recruited via a student employment website. The advertisement stated that the experiment "involved playing a game with three other participants using an alien language". It was specified that participants must have a high level of English (so that they would be able to understand the instructions without difficulty), and that none should have studied more than a semester of linguistics. All but six of the twenty participants were native speakers of English, and they came from a wide variety of academic backgrounds. They ranged in age from 18 to 42 . Participants were assigned to games on the basis of availability and to teams at random. ${ }^{5}$ Each participant was paid $£ 8$, and the members of the winning team in every game were awarded $£ 5$ each.

\subsubsection{Experimental procedure}

The procedure can be summarised as follows:

1. Participants arrive, sign consent forms, and choose cubicles;

2. Participants read instructions;

3. Training period: participants have ten minutes to learn alien language:

\footnotetext{
${ }^{5}$ There was one exception to this: two players in one game turned out unexpectedly to know each other. They were put, without their knowledge, on different teams
} 
(a) First five minutes: participants look at wordlists;

(b) Last five minutes: participants look at wordlists and cartoons;

4. Participants told about prize;

5. Participants play two practice rounds (with wordlists in front of them);

6. Participants play game proper (without wordlists);

7. Participants fill in feedback questionnaires;

8. Having been paid, participants leave.

Having read and signed consent forms, participants were seated in adjacent closed cubicles, each containing a computer (see Figure 5.1 for a diagram of the setup). They then read through instructions, presented to them on the screen; they were given as much time as they wanted for this. Having finished reading the instructions, and having been given a chance to ask any questions they might have, every player was given an identical list of English words with translations into the "alien language". They were given ten minutes to learn these alien words in any way they liked that did not involve them speaking out loud. They were also permitted to write on the wordlists, and were given pens for this purpose. For the last five minutes of this training session, they were also given access, on the screens in front of them, to a series of cartoons of people using the alien language (see Appendix B). ${ }^{6}$

After this training, participants were told about the prize available for winning the game (the advertisement had also mentioned a potential £5 "bonus" for success) and that they would now play two practice rounds, whose score did not count towards the game proper. For these two rounds, players were told they would be paired with their teammates. The chatting stage in the practice rounds also lasted 30 seconds longer than in the game proper, and players were allowed to keep their wordlists to refer to. Apart from giving the players a chance to get used to the game, and to improve their command of the alien language before the wordlists were taken away, these practice rounds (together with the training session described above) were intended to go some way to simulating childhood. The game proper, where players could not refer to their wordlists, but were told again that they would be

\footnotetext{
${ }^{6}$ They were not shown these for the whole ten minutes because pilots had suggested that — while the cartoons helped participants learn the language, or at least feel more comfortable with using it-being presented with the cartoons from the start distracted them from learning the words.
} 


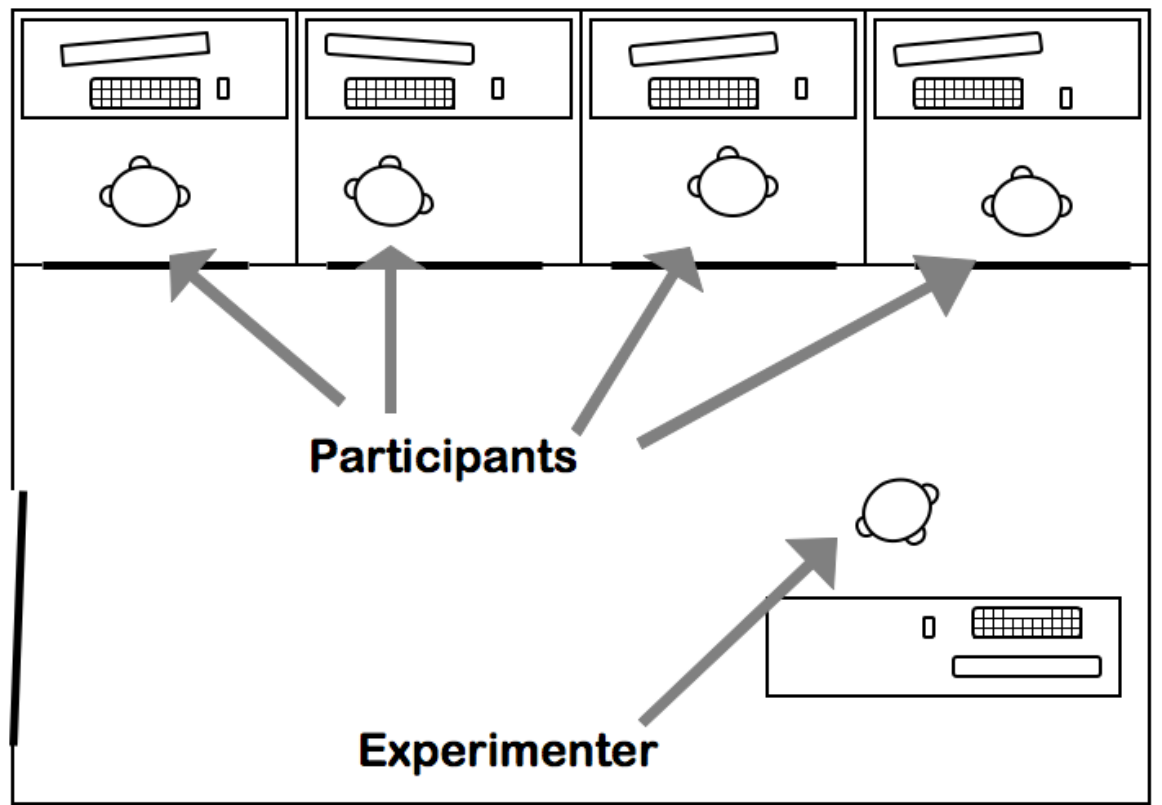

Figure 5.1: Diagram of experiment setup

paired with their teammates for the first two rounds, can be seen as simulating adolescence and adulthood. Section 5.6 will discuss the potential shortcomings in this approach, particularly the high level of contact between teammates early on in the game.

After the practice rounds, the players were given a minute to relax and have a last look over their wordlists. These were then taken away, and players began the full game, which lasted for 14 rounds (players were not told how many rounds to expect. See Section 5.2.4 for a detailed description of how the rounds were structured). As noted above, players were told in advance that they would be paired with their teammates for the first two rounds. In fact, they were paired with their teammates for the first three rounds. This was designed to frustrate players' likely expectations that, after two rounds with teammates, the next partner would most likely be an opponent (see Section 5.6 on the shortcomings of this). After this round, players were paired with their teammates in four more rounds, and with their opponents in seven (three rounds with one and four with the other). The order of these rounds was randomised, but no player was paired with any other player for more than three rounds in a row. This meant that, overall, players were paired an equal amount of 
time with both teams, but were paired with their teammate more than with any other individual player.

At the end of the game, participants were given a feedback questionnaire to fill in (see Appendix A).

\subsubsection{Game structure}

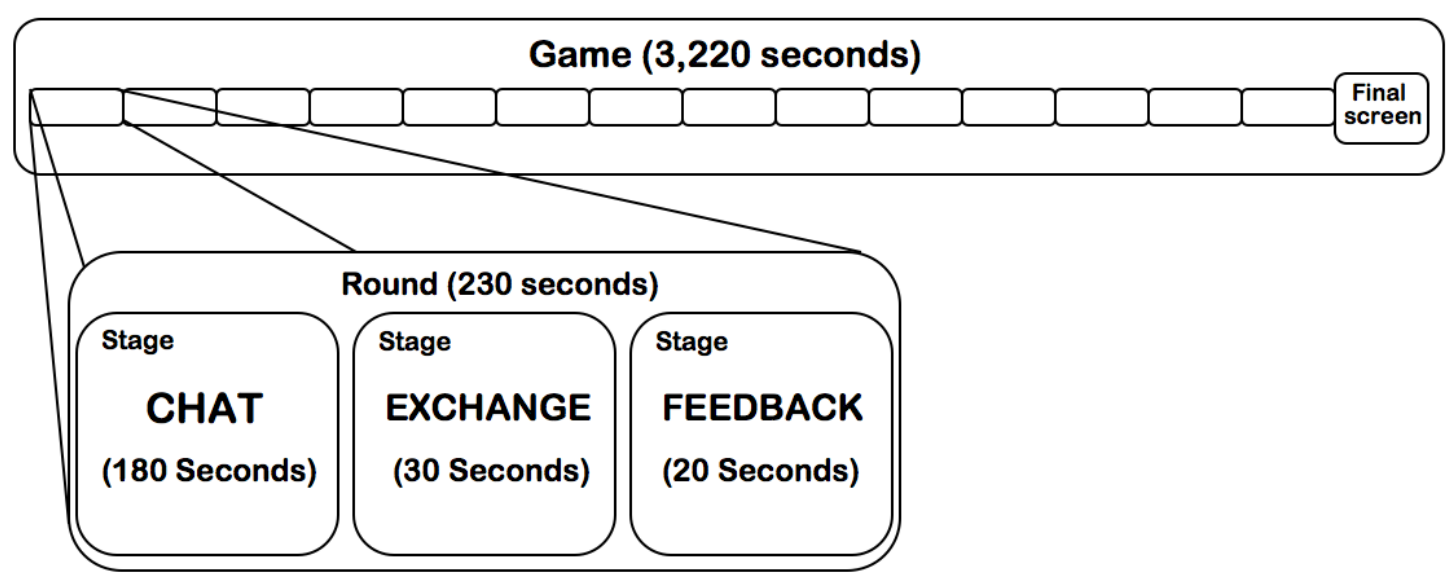

Figure 5.2: Diagram of game structure

Figure 5.2 is a diagram of the game structure. As noted above, a game consisted of a series of fourteen timed rounds. Players began the game with seven points, divided between five resources (see Section 5.2.5). Throughout a round, players could see their available resources on the left of the screen, and these were updated after the second stage if they gave anything away to, or received anything from, their partner.

Each round consisted of three stages. The first (CHAT) stage lasted for 180 seconds in the game proper and 210 seconds in the practice rounds (which otherwise did not differ). This was the stage in which players negotiated, by typing messages in the alien language, to exchange resources. They could do nothing to interact with their partner during this round except type and receive messages. This interaction worked using an instant-messenger-style program: either player could type a message at any time and, once they pressed enter, this message would appear on both their partner's screen and their own. Turn taking was not otherwise constrained. See 
Figure 5.4(a) for a screenshot (larger screenshots can be found in Appendix A in the instructions for participants).

This stage was followed by the EXCHANGE stage (see Figure 5.4(b)), which lasted 30 seconds. In this stage, players could no longer send messages, but they could choose to give away resources to the other player. They were free to give as little or as much as they wanted, provided they had enough to do so. To give away resources, players clicked on a button marked "+" for the relevant resource as many times as they needed to. If they wanted to change their mind, there was also a "_" button (nothing was final until the 30 seconds were up). Any resource given away was worth double to the receiver (cf. Nettle and Dunbar 1997), so, as the aim of the game was for one's own team to finish with more points than one's opponents' team, there was an incentive to give resources away to one's teammate, but not to opponents. In this stage, players were also asked, "Were you talking to someone on your own team?" and could answer on a scale of 1 to 4 , where 1 meant "Definitely not" and 4 meant "Definitely". They were told in the instructions that their answer to this question did not affect the game itself.

This stage was followed by a 20-second FEEDBACK stage (see Figure 5.5(a)), in which players were reminded what, if anything, they had given their partner, and told what, if anything, their partner had given them. They were also told if their partner had been on their team or not (but not any more specific information, such as, "You were talking to player A"). After this stage, either a new round started, or the game ended.

The final screen of the game showed, along with the player's own resources on the left of the screen (which had been visible throughout the game), the resources the player's team had managed to gather and the resources their opponents' team had managed to gather (see Figure 5.5(b)). It also gave the provisional score for each team, which equalled the sum of the team's shared resources, and the final score, which equalled the provisional score minus a penalty. This penalty was equal to the difference between the team's highest scoring resource and their lowest scoring resource. For example, if a team had amassed 100 points of meat, 80 points of grain, 60 points of water, 40 points of fruit, and 20 points of fish, then their overall 


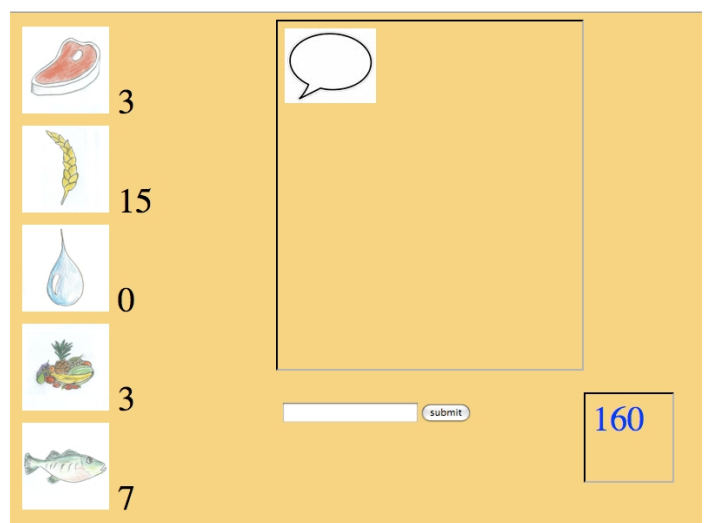

(a) CHAT Stage (in which players negotiate to exchange resources)

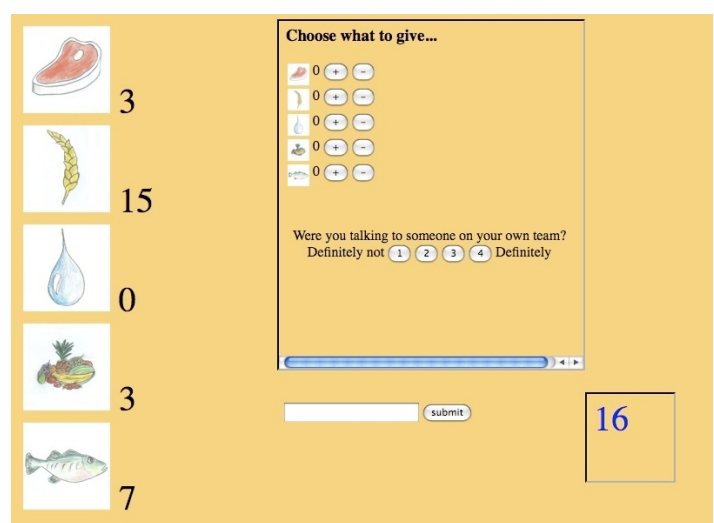

(b) EXCHANGE Stage (in which players exchange resources)

Figure 5.3: Examples of CHAT and EXCHANGE stages
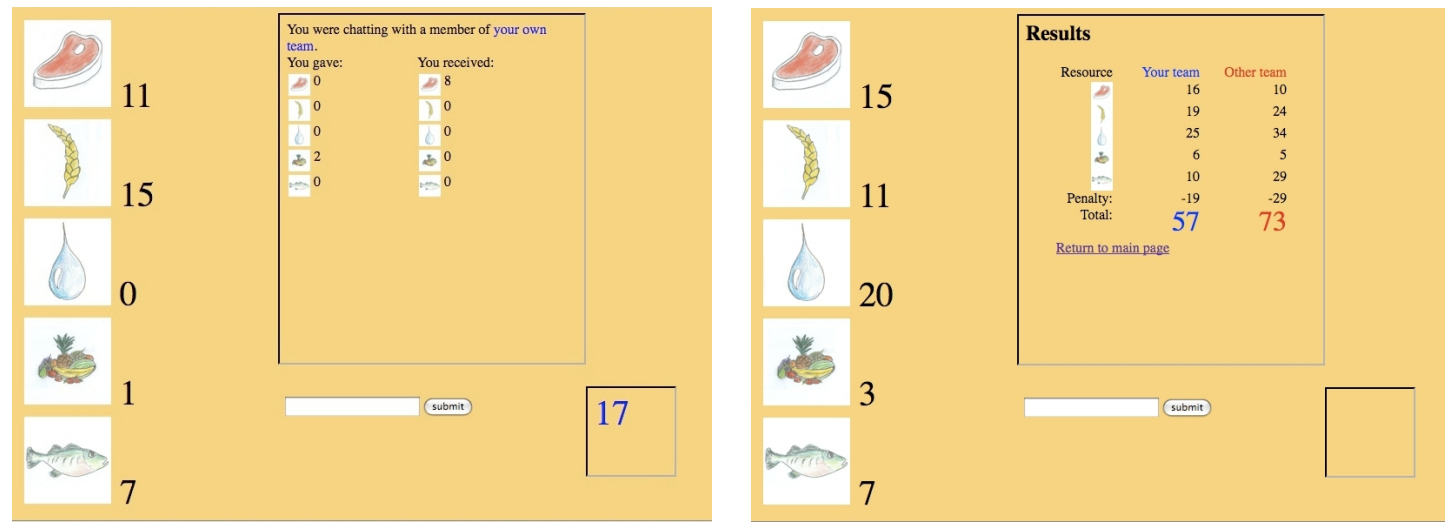

(a) FEEDBACK stage (in which players receive (b) Final screen (shows combined team scores feedback on exchange and interlocutor's iden- at end of game) tity)

Figure 5.4: Examples of FEEDBACK stage and final screen

score would be $300-80=220$ (since the difference between their score for meat and their score for fish would be 80). Neglecting one resource could therefore be very serious. Players were made aware of the penalty before the game started. Its main purpose was to ensure that there was material for negotiation in the CHAT stage. Otherwise, there would be no point in asking for more of any particular resource.

\subsubsection{Resources and scoring}

Players began the game with seven points each, divided between five resources: meat, grain, water, fruit, and fish. Every player began the game with three points in one resource, two points in one resource, one point each in two resources, and 
no points in the remaining resource. These quantities were allocated to specific resources at random, with the constraints that no two players in the same game had precisely the same distribution, and that the resource in which a given player had no points would be the resource in which their teammate had three points (therefore, no team in any game had fewer than two points between them in any resource). As mentioned above, there was a penalty applied to teams' scores at the end of the game, which equalled the difference between the team's highestscoring and lowest-scoring resources. The difference between resources had no more significance than this, however, and points in one resource were worth inherently no more or less than points in any other resource.

Throughout the game, players could see how many of a given resource they had available, but not how many their team had as a whole. At no time did any player see how many points any other player had, except at the end of the game, when the final combined scores for both teams were revealed.

\subsubsection{Alien language}

Players were not allowed to use English, any other real language, or numerals in the experiment. Instead they were given an "alien language" to learn, which consisted of nineteen randomly generated words of either two or three CV syllables. The alien words were generated anew for every game, and the length of the alien word (i.e. whether it had two or three syllables) paired with a given meaning was varied between games. The following is an example set, with English glosses:

$$
\begin{array}{ll}
\text { hello }- \text { jezife } & \text { want/need }- \text { hizu } \\
\text { goodbye - zemoze } & \text { give }- \text { howapi } \\
\text { please - zoyi } & \text { meat }- \text { bileja } \\
\text { thanks - saje } & \text { grain }- \text { jata } \\
\text { yes - fayide } & \text { water }- \text { tomuje } \\
\text { no/not - kiba } & \text { fruit }- \text { yinu } \\
\text { and - rupo } & \text { fish }- \text { sipuno } \\
\text { I - muba } & \text { much/a lot of }- \text { woduko } \\
\text { you - bulifo } & \text { question word }- \text { lani } \\
\text { have }- \text { mijeke } &
\end{array}
$$


The players were not given detailed instructions with regard to syntax, but, as mentioned in Section 5.2.3, they were shown cartoons of characters using the alien language (see Appendix B). All the sentences in these examples had an SVO structure, and the "question word" preceded the subject. There was, however, some variation introduced. First, three out of five sentences had overt subject pronouns, while these were lacking from the remaining two; second, one of the five sentences had a preverbal negative particle, while another had one post-verbally.

So that players would remain focused on the game and the screen in front of them, and so that memory lapses (along with typing mistakes) would provide a source for "natural" variation in the language, analogous to production errors in real language, players were not able to refer to their wordlists during the game itself and had to use the alien language from memory. Early pilots had suggested that participants felt somewhat overwhelmed by the prospect of having to "get the alien language right", so participants were also told not to...

... worry at all about making mistakes in the language. The important thing is to make yourself understood without using English (or any other real language).

This was also intended to encourage players to communicate as naturally as possible, and not to treat the task as a memory test.

\subsection{Summary of terms}

The design of this experiment is relatively complex, so this section will summarise the terms used to describe it, along with some details of the experiment.

Alien language An artificial language that PLAYERs had to use to communicate with each other during the CHAT STAGE of a ROUND. They were not permitted to use any real language, or to use numerals.

Final score The PROVISIONAL SCORE minus the PENALTY for a given TEAM at the end of a GAME.

Game The experimental task, engaged in by a GROUP of four PLAYERS. First the players would play a small number of PRACTICE ROUNDS, and then the GAME PROPER, which, in this experiment, lasted for 14 rounds. 
Game proper Those rounds of the GAME, following the PRACTICE ROUNDS, for which PLAYERS could be rewarded depending on their success.

Gift Points given away by a particular PLAYER during the EXCHANGE STAGE of a ROUND.

Group The four PLAYERS engaged in playing a given GAME. There were five groups in all.

Opponent A member of the same Group, but not the same TEAM. Every PLAYER had two opponents.

Participant An individual taking part in this experiment. There were 20 participants in all in this experiment.

Partner For the duration of every ROUND, each PLAYER was paired with one of the other players, who might or might not be on the same TEAM. This was their partner for that round.

Penalty The difference, at the end of the GAME, between the highest- and the lowest-scoring RESOURCE for a given TEAM. This was deducted from the PROVISIONAL SCORE to give the FINAL SCORE.

Player A PARTICIPANT described with respect to a particular GAME. There were four players in each game.

Practice round In this experiment there were two of these, and they were played before the GAME PROPER began, to allow PLAYERS to practise using the ALIEN LANGUAGE and playing the game. Scores from the practice round were not carried over into the game proper.

Provisional score This was equal to the sum of all RESOURCES belonging to a given TEAm. The PENALTY was deducted from this to give the FINAL SCORE.

Resource Every PLAYER started the game with seven points, split among five resources: meat, water, grain, fruit, and fish. No resource was worth inherently any more than any other.

Round A subdivision of a GAME, consisting of three STAGES.

Stage A subdivision of a ROUND. There are three stages in each round:

Chat stage In this stage, a PLAYER could interact with their PARTNER for the round by typing and receiving messages. They cannot, however, give 
any RESOURCES away. This stage lasted 180 seconds in this experiment (or 210 seconds in the PRACTICE ROUNDS).

Exchange stage In this stage, players could choose to give away some of their resources to their partner. This stage lasted for 30 seconds in this experiment.

Feedback stage In this stage, players were reminded what they had given away in the EXCHANGE STAGE, told what they had received from their partner, and told if their partner had been on their TEAM or not. This stage lasted 20 seconds in this experiment.

Proportional gift A GIFT expressed as the proportion of the giver's RESOURCE total at the time of giving.

Team In each GAME of this experiment, the four PLAYERS were divided into two teams of two. The object of the game was for one's own team to score more than the other team.

Teammate The other member of one's own TEAM. Every PLAYER had one teammate.

Variable set In the analysis of how the ALIEN LANGUAGE was used (see Section 5.4.2), a variable set is a set of words used in a particular GAME with a particular meaning (e.g. all the words used by players of one game to refer to "meat").

\subsection{Results}

Table 5.1 summarises the results for this experiment. The second game was unfortunately cut short by power failure in the middle of the ninth round. Data from this game will be included in the following sections only where it is practical to do so, and, in such cases, results excluding this data will also be given.

As noted in Section 5.2.1.2, there were two main dependent variables in this experiment:

1. Players' success in distinguishing between teammates and opponents.

2. The degree to which the alien language diverged. 
As will be discussed in more detail in Section 5.4.1, players did significantly better than chance at identifying their teammates. As will be shown in Section 5.4.2, players were also significantly closer to their teammates than to their opponents in their use of the alien language, indicating that the language was beginning to diverge into team "dialects". Section 5.4 .3 will look at how teams' scores correlate with other factors, and Section 5.5 will discuss the particular strategies the players employed, and how these relate to divergence. In these sections, teams will be referred to by a number and a letter; for example, "Team $3 b$ " refers to Team b in the third game.

\begin{tabular}{|c|c|c|c|c|c|}
\hline & $\begin{array}{l}\text { Final } \\
\text { score }^{a}\end{array}$ & $\begin{array}{l}\text { Convergence } \\
\text { level }^{b}\end{array}$ & $\begin{array}{l}\% \text { Correct } \\
\text { recognitions }\end{array}$ & $\begin{array}{l}\text { Mean } \\
\text { gift }^{d}\end{array}$ & $\begin{array}{l}\text { Strategies } \\
\text { employed }^{\mathrm{e}}\end{array}$ \\
\hline \multicolumn{6}{|l|}{ Game 1} \\
\hline Team a & $35(47)$ & 0.91 & $79.2(24)$ & $1.3(1.8)$ & $\mathrm{DR}, \mathrm{M}$ \\
\hline Team b & $33(46)$ & 0.95 & $79.2(24)$ & $1.1(1.5)$ & $\mathrm{DR}$ \\
\hline \multicolumn{6}{|c|}{ Game $2^{\mathrm{f}}$} \\
\hline Team a & $52(61)$ & 0.86 & 90.9 (23) & $2.1(4.1)$ & DR, II \\
\hline Team b & $13(17)$ & 0.91 & $66.7(24)$ & 1.1 (1.3) & DR \\
\hline \multicolumn{6}{|l|}{ Game 3} \\
\hline Team a & $41(55)$ & 0.90 & $75(24)$ & 1.5 (2.3) & $\mathrm{DR}, \mathrm{M}$ \\
\hline Team b & $28(44)$ & 0.88 & 66.7 (24) & $1.1(1.5)$ & $\mathrm{DR}, \mathrm{M}$ \\
\hline \multicolumn{6}{|c|}{ Game 4} \\
\hline Team a & $54(67)$ & 0.89 & 73.9 (23) & $1.8(2.7)$ & $\mathrm{DR}, \mathrm{M}$ \\
\hline Team b & $52(82)$ & 0.84 & 77.3 (22) & $2.5(4)$ & $\mathrm{DR}, \mathrm{M}, \mathrm{II}, \mathrm{SH}$ \\
\hline \multicolumn{6}{|c|}{ Game 5} \\
\hline Team a & 432 (463) & 0.88 & 85.7 (21) & 16 (31.8) & DR, M, II, SH \\
\hline Team b & $28(35)$ & 0.94 & $75(24)$ & $0.8(1.2)$ & $\mathrm{DR}, \mathrm{M}$ \\
\hline
\end{tabular}

Table 5.1: Summary of results from first experiment.

${ }^{\text {a }}$ Provisional score given in brackets;

b 1 - mean distance between teammates (higher figure means greater convergence); see Section 5.4.2;

${ }^{c}$ Excludes first two rounds and rounds where players made no response; number of responses made given in brackets (out of a possible 24);

${ }^{\mathrm{d}}$ Based on all rounds; mean gift to teammates only given in brackets;

e See Section 5.5.1. DR = Dialect Recognition; $\mathrm{M}=$ Mimicry; II = Intentional Innovation; $\mathrm{SH}=$ Secret Handshake;

f This game cut short by power failure. 


\subsubsection{Recognising teammates}

In the feedback questionnaire, participants were asked if they had been able to recognise their teammates and, if so, how. All but one said that they had, at least some of the time; of these 19 participants, 18 reported basing these judgements on linguistic cues. Both members of Team 4 a reported that this became more difficult towards the end of the game, however, as their opponents had started mimicking their use of language (members of Team $4 \mathrm{~b}$ confirmed their use of this tactic in their own questionnaires). The one player who claimed some success in recognising teammates, but did not mention linguistic cues, reported basing his judgements on "response time ${ }^{7}$ and the items they have or don't have". Two other players made reference to recognising teammates based on the resources they claimed to have, but only in addition to linguistic cues. Section 5.5.1 will discuss in detail the linguistic strategies that players employed.

Aside from the players' own subjective impressions, there are two quantitative measures of how well they did at recognising their teammates. The first measure comes from their having been asked explicitly. In the second stage of each round in the competitive condition, players were asked: "Were you talking to someone on your own team?" and answered on a scale of 1 to 4 , where 1 meant "Definitely not" and 4 meant "Definitely". The third column of Table 5.1 shows the percentage of correct recognitions for each team, where an answer of 1 or 2 is taken to mean "no", and an answer of 3 or 4 is taken to mean "yes". Responses from the first two rounds, where players knew they were talking to their teammates, were ignored, as were rounds where players neglected to respond (which make up $2.9 \%$ of potential responses). As can be seen, players were more often right than wrong. Of the 216 judgements made, only 56 were incorrect, which is highly significant $(p<0.001$, based on a one-tailed binomial sign test). If the results from Game 2 are excluded, the results are similarly significant $(p<0.001 ; S=44$ incorrect out of 186). Definite guesses (i.e. a guess of 1 or 4) were, unsurprisingly, far more often right than wrong (97 correct against 14 incorrect).

\footnotetext{
${ }^{7}$ He gave no further details about this. Response time may, of course, be regarded as a linguistic variable, at least to an extent. It stands in contrast, however, to the much more unambiguously linguistic markers that other players referred to, such as punctuation, spellings and greeting forms.
} 
One problem with this measure, of course, is that players sometimes neglected to answer the question. As there were four players playing at any one time, and the rounds were timed, they could not be made to answer the question without interrupting the game. In this case, it is unimportant. First, what players in fact thought in these rounds can be inferred with reasonable expectation of accuracy from their giving behaviour on these rounds (see below). Second, as failures only constituted $2.9 \%$ of responses, the results remain significant however these are interpreted. An interesting question remains, however: where players are asked explicitly what they think, their responses will be one step removed from their actual impressions of who they were talking to, which might be filtered through post hoc justifications, reconsiderations, and the like. It is, moreover, possible that players might subconsciously respond to something in an interlocutor's use of language, but consciously come to a different conclusion on more rational grounds. Of course, it might be possible to get some interesting data on this through neuro-imaging. ${ }^{8}$ While this was not done, the question is not raised for the sake of idle speculation. A second measure of players' impressions is available, which can be compared with the explicit responses. This is gift size, which, as noted above, could be used to infer what players thought in those rounds where they failed to answer the question. Since any points given away were worth double to the receiver, we should expect that gifts to teammates should be larger than gifts to opponents. There is one caveat to this: given the pressure to keep resources balanced, if players had rather too much of one resource, it might seem a reasonable strategy to give much of it away to an opponent, particularly if that opponent also claimed to be overburdened with that resource - a strategy that will be referred to here as "malign generosity". Indeed, two players claim to have done this, but one of these mentioned it only as an occasional alternative to giving nothing; the other eighteen players claimed to have tried to avoid giving anything to opponents.

To test whether players treated all assumed opponents the same, gifts were compared (as a proportion of the player's resources at the time of donation) for those rounds where they voted no: if players were talking to teammates, did they give them more than they gave their opponents, despite explicitly guessing "opponent"

\footnotetext{
${ }^{8}$ Such methods are not unusual, for example, in studies of multilingual processing; de Bot (2008) gives a relatively recent, though pessimistic, review.
} 
in both cases? ${ }^{9}$ The mean proportional gift to teammates on these rounds (0.02) was in fact higher than the mean proportional gift to opponents (0.01), but a Wilcoxon $T$ test (where $N_{1}=35$ and $N_{2}=98$ ) showed that this was not significant $(T=1923, p>.05)$. Players' gifts in these games were thus in line with their explicit judgements of whether or not they were paired with teammates. Everything, in other words, indicates that players of this game were able to use linguistic cues, in a highly restricted artificial language they had only just learnt, and which was initially identical for all of them, to recognise people they had barely met.

\subsubsection{Divergence}

The previous section described how players managed to recognise each other using markers arising in the alien language. One of the main research questions behind this experiment was whether this would cause the language to start diverging into team "dialects". For this to happen, variation must arise between players (it will be recalled that all four players in a game learnt the same alien language). This alone is not enough, however. The development of four idiolects would not be of the greatest interest (see Section 3.2). Far more interesting if there were also structured convergence: if players were closer to their teammates, in their use of the language, than to their opponents.

That there was variation in the players' use of the language is easy to observe, and a large amount of it emerged during the game. If we consider only the four games for which there is full data, there are 76 potential variables (19 initial words $\times 4$ ); alongside these, the players introduced 282 new variant forms. The vast majority of this variation appears to have arisen accidentally, as a result of typing mistakes, poor memory, and so on: most players denied making any deliberate changes (as can be seen from the fifth column of Table 5.1, members of only three teams claimed to have employed Intentional Innovation as a strategy; see Section 5.5.1). There were, moreover, a few variables that did not produce any new variants. If these are ignored (since the only variables that matter for the measurement of between-player

\footnotetext{
${ }^{9}$ The second game will be excluded from this analysis, as the data was too incomplete to be helpful.
} 
variation are those with more than one variant), then there were 6.2 variants used for every variable.

Given this variation, players could be compared based on the relative frequency with which they used particular variants. To this end, every individual word-form used in every one of the four completed games was extracted along with a frequency count for each player (i.e. how many times each player used a given form) and sorted into "variable sets", according to which players could be compared. Variants were assigned to these sets based on formal similarity (see, for example, the variants in Table 5.2) and the context in which they were used, which allowed their intended meaning to be inferred.

For instance, in a particular game, one player might use only the form hizu to mean "want", while another player might use hizu sometimes and (the innovative form) hizo the rest of the time. These - along with any other forms used to mean "want" in a given game - would constitute a variable set. On the basis of these sets, pairs of players could be compared according to which variant they favoured. This is analogous to variation in real language, where speakers vary in the phonetic forms they use (e.g. the word "meat" might be pronounced variously as [mit], [mi?], [mit], [mər?] etc.). All variants were on the word level; variation in capitalisation was included ( $g a z o$, GAZO, Gazo and gAzo, for example, would be treated as four separate variants), but syntactic variants were not. There was a particular kind of morphological variant, however, which was a special case. This is where the repetition of individual letters was used to indicate number. For example, Team 5a used ttttt to mean "5 points of meat [i.e. tasobo]". In such cases, ttt, ttttttt and ttttttttttttt etc. were not all treated as separate variants, but as one. For each of these variable sets, the raw frequency scores were converted to proportions. The mean Euclidean distance between each pair of players was then calculated. Table 5.2 shows an example, taken from one game, of a variable set consisting of five variants. The figure on the left of each cell is the raw frequency and the figure in brackets is the proportion. The form rohapi was given to participants in the word list at the start of the game, and glossed as "want/need". The other variants can be assumed to have arisen through error. Players 1 and 2, as can be seen, showed a strong preference for the original variant. Players 2 and 3, however, both used the 
metathesised form ropahi almost as frequently. Other forms were also used once or twice. Table 5.3 shows the mean distance (based on the proportions) between every pair of players for this example set; the smallest distances are marked in bold.

\begin{tabular}{lllll}
\hline & \multicolumn{2}{c}{ Team 1 } & \multicolumn{2}{c}{ Team 2 } \\
& Player 1 & Player 2 & Player 3 & Player 4 \\
\hline rohapi & $34(.97)$ & $27(.93)$ & $11(.52)$ & $10(.48)$ \\
roahpi & $1(.03)$ & $2(.07)$ & $0(0)$ & $0(0)$ \\
ropahi & $0(0)$ & $0(0)$ & $9(.43)$ & $9(.43)$ \\
rophui & $0(0)$ & $0(0)$ & $1(.05)$ & $0(0)$ \\
wohapi & $0(0)$ & $0(0)$ & $0(0)$ & $2(.09)$ \\
\hline
\end{tabular}

Table 5.2: Example variable set from first experiment

\begin{tabular}{lllll}
\hline & \multicolumn{2}{c}{ Team 1 } & \multicolumn{2}{c}{ Team 2 } \\
& Player 1 & Player 2 & Player 3 & Player 4 \\
\hline Player 1 & 0 &. $\mathbf{0 2}$ & .192 & .208 \\
Player 2 & $\mathbf{. 0 2}$ & 0 & .192 & .208 \\
Player 3 & .192 & .192 & 0 & $\mathbf{. 0 3 6}$ \\
Player 4 & .208 & .208 & $\mathbf{. 0 3 6}$ & 0 \\
\hline
\end{tabular}

Table 5.3: Mean distances between players in example set

The second column of Table 5.1 shows the mean level of convergence for every team (calculated as 1 minus the mean distance between teammates across variable sets; a score of 1 would indicate complete convergence). In no game was there a significant between-team difference in within-team convergence (based on a Monte Carlo test). This fact should not be misinterpreted. It means only that all players converged linguistically with their teammates to a similar level. It is important to note that this is not a measure of between-team divergence: if all four players in a game used the language in exactly the same way, both teams would show the same level of convergence; on the other hand, two teams could show the same level of convergence, yet diverge significantly from each other. To test whether teams had diverged in any condition, the variable sets were scored: if, for a given variable set, every player was closer to their teammate than to either of their opponents (which is the case in the example set; see Tables 5.2 and 5.3), the set was awarded a score of 1 (meaning that the teams had diverged with regard to this variable). If, on 
the other hand, this was true of neither team, the set was scored 0 (meaning that there was no between-team divergence). If both members of one of the teams were closer to each other than either was to their opponents, but this was not the case for the other team, the set was scored 0.5. The scores for all variable sets in all four complete games were then summed, to give an overall divergence score (in this case 19). $P$ values were calculated using the Monte Carlo method; that is, the data was shuffled and rescored 10,000 times (see Appendix C for more details of this approach), and the score was found to be highly significant $(p<.001)$, indicating a significant tendency towards socially, and geographically, ${ }^{10}$ structured divergence in the alien language.

\subsubsection{Syntactic variation}

The measure described above involves only word-level variants, but can be seen as including morphological, phonological, and phonetic variation, to the extent that these levels of structure played a role (see Section 5.4.2 for an example of something like reduplication; orthography acted as a proxy for sound). This leaves syntax. To investigate syntactic variation, every utterance used in the game was extracted and rewritten as parts of speech. For example, a sentence meaning, "I give you meat fish water" would be represented as, "PRO VERB NOUN NOUN NOUN". Players were compared on three variables: position of pronouns, position of the interrogative particle (glossed as "question word" in the wordlists), and position of negative particles in sentences with verbs. There were three variants for the second two variables: left, right and on both sides. The same variants were measured for pronoun position, but this variable had an extra variant: sentences with a verb, but no pronoun at all (as was noted in Section 5.2.6, this possibility was presented to the players in training, as were both pre- and post-verbal negative markers). Figure 5.5 gives the frequency of the different sentence types. Players were compared for these variables as they had been for word-level variants. In this case, however, there was no significant divergence $(p=.25)$.

\footnotetext{
${ }^{10}$ With frequency of interaction understood as a proxy for geographical distance.
} 


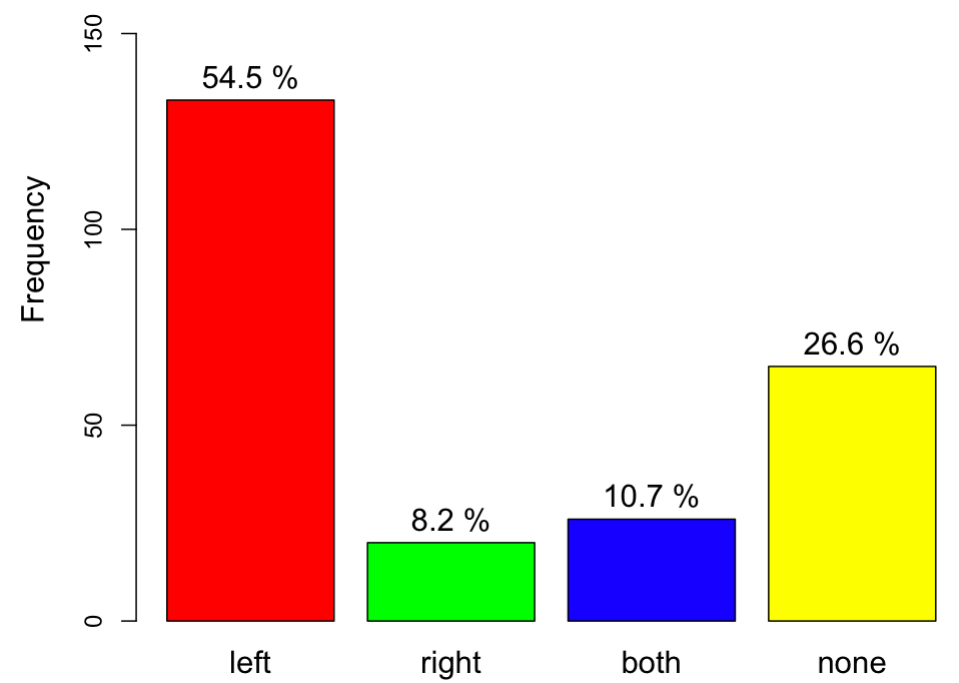

(a) Position of pronoun

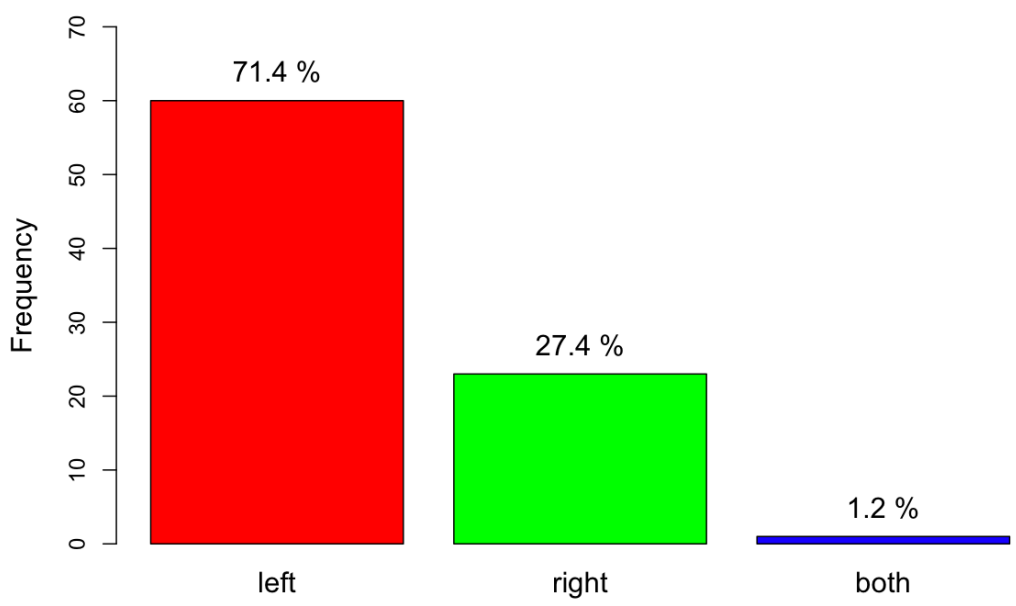

(b) Position of negative marker

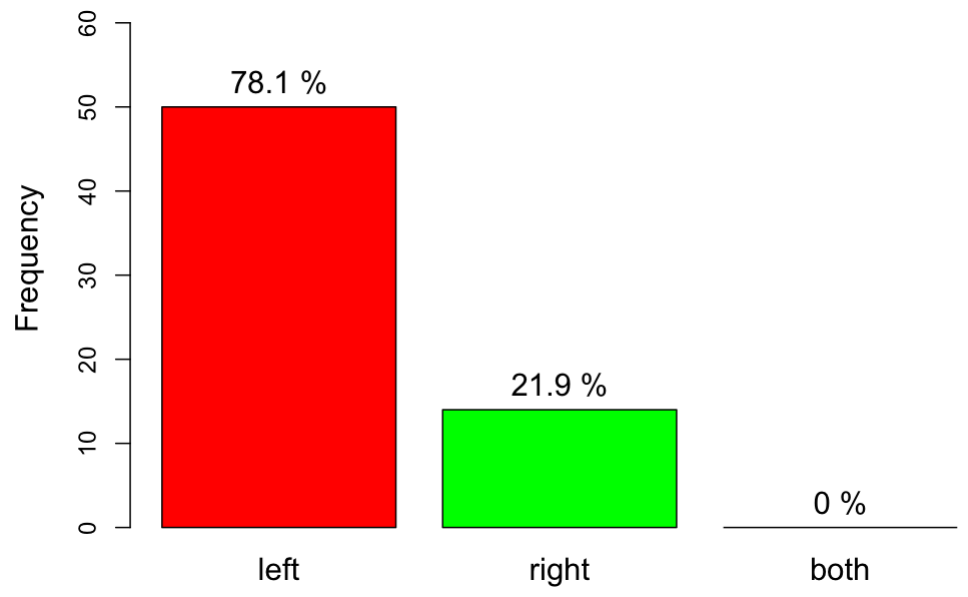

(c) Position of interrogative marker

Figure 5.5: Frequency of sentence types 


\subsubsection{Comparison with score}

In three out of the five games, the team that most often distinguished correctly between teammates and opponents also won the game. In one of the two games in which this was not the case, both teams did equally well at distinguishing, and their scores were also extremely close, differing by only one point before the penalty was applied, and by only two points afterwards. In the other of the two games, the team with the best recognition rate actually scored 15 points more than their opponents before the penalty was applied, but lost by two points after it had been. The team producing the most definite guesses (1 or 4$)$, which was also always the team with the most correct definite guesses, attained the highest provisional score in every game. While this all might suggest that recognition rate was quite a good predictor of (pre-penalty) success, there was no significant correlation between team recognition rate and provisional score $(r(8)=-.465, p>.05)$. While recognition rate is a good predictor of the winning team, absolute score is the result of several other factors, which will be discussed in Section 5.5.

Similarly, there was no correlation between the level of within-team convergence and score ${ }^{11}(r(8)=-.25, p>.05)$ and, as noted in Section 5.4.2, there was no significant difference between levels of within-team convergence in any game. On the face of it, this contradicts expectations. It should not, however. For teammates to be able to identify each other, an especially high level of convergence is not necessary; nor is it necessary to be any more converged linguistically than one's opponents. As noted in Section 5.4.2, within-team convergence is not even a good predictor of between-team divergence. For the purposes of recognising one's teammate, one suitably salient variant or shared signal is sufficient for recognition. As can be seen from the fifth column of Table 5.1, the two highest-scoring teams (before penalty) developed a very powerful code: a "Secret Handshake" (see Section 5.5.1). This was the strategy that led them to be so successful. ${ }^{12}$

\footnotetext{
${ }^{11}$ Either provisional or final; the coefficient given is for provisional score, as this is likely to be the most meaningful measure for these purposes.

${ }^{12}$ At least in the case of Team 5a. Team 4b developed their Secret Handshake rather too late in the game; they let themselves down, moreover, by allowing their resources to get dangerously unbalanced. However, had they developed the handshake earlier, their penalty is unlikely to have mattered. Team 5a incurred the largest penalty of any team, but still beat their opponents by 404 points!
} 
The overall levels of within-team convergence and between-team divergence are better treated as by-products of adaptive strategies rather than as strategies themselves. Section 5.5 discusses the strategies that players actually used.

\subsection{Strategy}

\subsubsection{Identification strategies}

The linguistic strategies available to players to identify, and be identified as, teammates can be summarised as follows: ${ }^{13}$

- Dialect Recognition (DR): try to remember how different players use the language and identify them based on this. For example, a player in Team 1a reported noticing that her teammate did not use first-person pronouns with verbs.

- Intentional Innovation (II): make changes to the language that are likely to be recognisable. For example, players in Team 2a and Team 5a in started abbreviating resource names and repeating the abbreviations to indicate number.

- Mimicry (M): try to sound as much like the person you're talking to as possible. For example, in both Game 2 and Game 5, the players who started abbreviating resource names were soon imitated by their teammates. Players in several games also claimed to have intentionally imitated the linguistic behaviour of their opponents (Team 5a did this in greetings; see below)

- Secret Handshake (SH): say something at the beginning of the interaction that identifies you to your teammate (and ideally not your opponent), and requires a specific response from them (see below for an example).

The strategies are referred to in abbreviated form in the last column of Table 5.1. Inclusion in that column is based on players' own reporting in feedback questionnaires of the strategies they employed and, where this can be clearly seen, evidence in the data.

\footnotetext{
${ }^{13}$ As mentioned in Section 5.4.1, three of the twenty players claimed to have attempted to keep track of their teammates' resources (based on what they offered and asked for) so as to identify them on this basis. Since they did not report success at this, it will be ignored in the following analysis. It should also be noted that it is hard to identify cases of intentional mimicry in the data; this may therefore be underreported.
} 
There seems to be some sign of implicational universals in strategy use. For example, all teams employed Dialect Recognition, and most employed Mimicry as well. Only one team claimed to use Intentional Innovation, but not Mimicry (and it is possible that they simply neglected to report the latter strategy). This suggests that Dialect Recognition is the simplest (or at least the most obvious) strategy, and that Mimicry and Intentional Innovation are less basic, with some suggestion that the latter may be the less basic of the two, though this is less certain (and see Sections 6.4.1 and 7.3.1). No teams employed a Secret Handshake who did not also employ all three of the other strategies. The significance of this pattern should not be overstated, however. Apart from trying to keep track of the resources one's teammate's claims to have, which is clearly not likely to be very reliable, Dialect Recognition is the least a player of this game can do apart from doing nothing. It is clear, moreover, that Secret Handshake is the most complex strategy, since it involves coordination with another player; it also entails both Dialect Recognition (as players have to recognise that their teammate is doing something special) and Intentional Innovation (as players have to create a salient greeting).

As Table 5.1 shows, Secret Handshakes occurred in Games 4 and 5, and the teams that used it were the most successful in the game. Team 4b's Handshake had four parts. The word for "hello" in this game was zojo, and, as early as the seventh round, the two members of this team greeted each other as follows:

Player 3: zojo

Player 4: zojo

Player 3: zo

Player 4: zo

It was not entirely successful on this round, however, as neither member was convinced that the other player was on their team (Player 3 voted 2 and Player 4 voted 1). Precisely the same sequence was repeated in the tenth round (by which time Player 3 had also greeted Player 1 with $z o$, but with no response in kind). This time, Player 3 voted 4 (but gave only three points of meat). It was not until the thirteenth round that both members of the team worked out that they were on to something. Happily for them, they were also paired up for the fourteenth round, 
which allowed them to make even more use of their Handshake, but by then it was too late to develop it further.

Team 5a's Handshake was much more impressive. In this game, the word for "hello" was sekudi, but Team 5a did not use this between themselves. Instead, they used jori noloku, which literally meant, "I have". In early rounds (in fact, it began in the second Practice round), this seems to have been meant literally. Members of this team just dispensed with the ordinary greeting and got down to the business of stating what resources they had. Members of the other team, however, tended to begin with a greeting. This difference of approach soon became obvious to Team 5a, and by the seventh round they had turned it into something rather interesting:

Player 0: jori noloku

Player 0: ?

Player 1: jori nokolu tasabo tasabo vawu vawu vawu vawu ...

As indicated by Player 0's question-marked pause, jori noloku had become an identity marker to these players; this also meant that it was beginning to resemble a greeting-Player 0 did not immediately state what resources she had. A further marker will be noticed in the above exchange: players in this team repeated resource names to indicate how much of something they had. This was not especially unusual: Team $2 \mathrm{a}$ did this too, and Team $4 \mathrm{~b}$ also did it a little. Team $5 \mathrm{a}$ stood out, however, as engaging in this very early on. Player 1 started it in the very first Practice round, and this no doubt served as a reinforcing marker, along with jori noloku, by which to identify each other, and in which to ground the Handshake. Later in the game, when it became hard work typing everything out, they started abbreviating the resource names (Team $2 \mathrm{a}$ did the same). Towards the last rounds of the game it is not clear that there was much information being transmitted about the actual amounts these players had. The point seems more that it reinforced their bond.

Team 5a's Secret Handshake had other subtleties. Members of this team would wait before beginning an interaction; if their interlocutor started with sekudi, they would reply with the same, and imitate their opponent's language throughout the interaction. If, on the other hand, they judged that no sekudi was coming, they 
would begin with jori noloku. This backfired once, when Player 2 began the interaction, for some reason, with sekudi. As a testament to the quality of the handshake, neither thought the other was their teammate.

This team was unusual in its cleverness (and it seems the brains of the team was Player 1), although the similarities with real-life greetings rituals, ${ }^{14}$ and the Biblical "shibboleth"-story are striking. Nevertheless, it is equally striking that the vast majority of players reported success at identifying their teammates without resorting to such inventive strategies. Fascinating as they are, cases of such Secret Handshakes should be seen as exceptional, and should not distract excessively from the less salient successes of other teams.

\subsubsection{Giving behaviour}

The fourth column of Table 5.1 shows the mean amount of resources given away per round by each team, and the mean amount given to teammates (whether or not players guessed who they were paired with). The most successful teams were the most generous (apart from Team 4b, who did score more than their opponents before the penalty was applied). Indeed, there is a very clear - if unsurprising - correlation between mean gift and score $(r(8)=.998, p<.0005$; based on provisional score). In spite of this, most players were rather conservative in their gift-giving. Even in the first two rounds, when players were told explicitly that they would be paired with their teammates, no player gave away everything, and only two players ever gave away more than half their resources (these were the two members of Team 5a). There are three points to be made about such conservativeness. First, there are very few real-life situations where giving away all one's resources is a good idea, so it is possible that players were following a generally adaptive heuristic. Second, as the results of Game 4 show, it was important to keep resources balanced, and players knew it; this obviously encourages caution. Finally, there was a simple physical constraint: giving away resources involved clicking on a + button. This means that, the more resources one wishes to give away, the more effort is required. When the amounts involved are relatively low, as was the case early in the game,

\footnotetext{
${ }^{14}$ And not only those employed by secret societies. Even "how do you do?" has, for most of the people who use it regularly, a correct response that is not a direct answer to the question.
} 
this does not make much difference. Later in the game, however, particularly if players have already amassed a large number of resources, it becomes increasingly harder to give away a large proportion of one's resources. Given the time limit, there is obviously a ceiling on the amount that can be given away at all.

Nevertheless, only one team really rose high enough to hit this ceiling. This is Team 5a, whose members were the only players in any game to ever give away more than half their resources, and who also achieved by far the highest score. It should not be surprising that this generosity occurred only in the team that employed a Secret Handshake from early on. Not only does this powerful strategy create a more secure environment for generous giving, but it is itself costly to establish - that is, it is more complex, and requires more coordination, than any other strategy - and is thus a non-conservative behaviour.

\subsubsection{Summary}

The most successful strategy was a combination of generous giving and the intentional creation of salient markers consisting of more than one element. The latter, as a particularly complex strategy, takes effort to establish and is thus the costliest of the identification strategies, but once established, is relatively cheap to use. Generous giving is inherently risky, but a safer environment can be created for it by the Secret Handshake.

An important point must be borne in mind: most players engaged very little in either of these behaviours. The majority relied instead on recognising less salient patterns of variation (this has been referred to here as Dialect Recognition), some less complex innovation, mimicry, and a relatively conservative exchange of resources. It is this behaviour, not the more sophisticated Secret Handshake strategy that led to the divergence described in Section 5.4.2.

\subsection{Discussion}

This experiment showed that linguistic processes which take a considerable time in real life can be modelled in a laboratory setting, using real human participants. Not only did players succeed in bootstrapping linguistic identity markers in a new 
language in a very short time (under an hour), but their behaviour in the game led the language to begin diverging into two "dialects."

The results, in other words, were very promising, and this experiment can be seen as a useful proof of concept. Nevertheless, there were shortcomings. Perhaps the most obvious concerns the high level of within-team interaction at the start of the game. Players were told nothing about who precisely their teammates were, but they could not play entirely blind; for this reason, they were told that the first player they spoke to would be their teammate. However, as was mentioned in Section 5.2.3, this period of explicit within-team interaction was in fact quite extensive: players knew that they would be paired with their teammates for both practice rounds and for the first two rounds of the game proper. What is more, they were in fact paired with their teammates for the first three rounds. This was partly because it was assumed that players would need a relatively long period of interaction with their teammates to be able to play the game. Second, this long within-team interaction was supposed to simulate childhood, in which individuals are less frequently exposed to outsiders without supervision. Nevertheless, in retrospect, this was almost certainly unnecessary. The third round of the game was always between teammates because, having just spent two rounds with their teammates, players were expected to be biased towards guessing they were paired with an opponent in the third round (in fact, only seven of the twenty players guessed they were paired with their teammates in this round). However, this problem could have been avoided by having players explicitly paired with teammates only in the first round, which would most likely have been a perfectly sufficient opportunity for players to anchor themselves.

A similar issue was that players interacted more frequently with their teammates overall than with either of their opponents. Although they interacted an equal amount of time with members of each team, this meant interacting $50 \%$ of the time with one's teammate and no more than $29 \%$ of the time with either opponent. ${ }^{15}$ Although there is nothing wrong with this as such, it does not allow a clear distinction to be made between the pressure to mark identity and frequency of interaction

\footnotetext{
${ }^{15}$ As there were 14 rounds in the game, players did not interact an equal number of rounds with both opponents. This was another minor shortcoming to be remedied.
} 
as explanations for the linguistic divergence. A control condition, either with a lower frequency of interaction or without the pressure to mark identity, would be necessary to make this distinction.

Those are the two chief shortcomings of the experiment. There were also three smaller problems. First, this experiment did not entirely exclude participants who had studied linguistics; participants were admitted who had studied a semester or less of the subject. While it is unlikely that a semester of linguistics would make a significant difference, individuals who have decided to study linguistics are likely to be individuals with a particular interest in language and, perhaps, linguistic variation specifically, and should probably have been excluded. ${ }^{16}$ Second, a relatively high number of players neglected to say, in the EXCHANGE stage of the round, whether or not they thought they were paired with a teammate. Since the rounds were timed, it was impossible to enforce this strictly, but this indicated at least that the importance of doing so should be stressed more clearly. Finally, as mentioned in Section 5.4, one game was cut short by a power cut. This is not a shortcoming of the experimental design, but it at least gave more reason to replicate the experiment, incorporating improvements as suggested above. Chapters 6 and 7 describe two further, larger-scale, experiments that do just that.

\footnotetext{
${ }^{16}$ The importance of this should not be overestimated. It is certainly safer to exclude all students of linguistics from an experiment that concerns language divergence. However, it is far from clear that it makes a great difference. There are, after all, a host of potential biases that different participants might bring, not all of which are related to language. As has been suggested elsewhere (e.g. Sears 1986), we should be wary of taking the behaviour of Western undergraduate students as accurately modelling the behaviour of all human beings. This participants in this experiment included both undergraduate and postgraduate students, and the age range was relatively wide. However, it would be very interesting to try running the experiment with non-university-educated participants and with participants outside this age range.
} 


\section{CHAPTER 6}

\section{Controlling for the pressure to mark identity: a sec- ond experiment}

Chapter 5 described an experiment in which four participants played a game that involved using an artificial "alien language" to negotiate anonymously to exchange resources. The players were divided into two teams of two and the game was set up in such a way that it was advantageous to give away resources to one's teammate, but not to one's opponents. Owing to the anonymity of the interactions, players were forced to rely on the alien language as a source of social markers, and they successfully used them to identity each other. Over the course of the game, this led the alien language to start diverging into team "dialects".

However, as discussed in Section 5.6, it is difficult to be sure what caused this divergence: each player interacted more with their teammate than with either of their opponents, so frequency of interaction may well play an important role, or perhaps even the only role, in the divergence. Indeed, frequency of interaction must play some role: there must be a minimum level of interaction with one's teammate, both absolute and relative to opponents, that allows players to accommodate to them. This leaves two questions: what is this minimum level, and is it sufficient to account for the divergence, or is a pressure to mark identity necessary? The experiment described in this chapter attempts to go some way to answering these questions. It introduces a control condition in which there is no pressure to mark identity, and it equalises the frequency of interaction between all players, so there is no frequency bias towards interacting with one's teammate. A further problem 
of the first experiment, noted in Section 5.6, was the relatively high interaction between teammates in the first rounds of the game. This was significantly reduced in the experiment described in this chapter.

\subsection{Description of experiment}

\subsubsection{Overview}

This experiment was carried out in May and June 2008, and involved forty participants, who were divided into ten groups of four. Each of these ten groups played a computer game. Five of the groups played a competitive version of the game, for which they were divided into two teams of two; the object of this game, as in the game described in Chapter 5, was for one's own team to score more points than the other team. In this case, however, players were also encouraged to beat their opponents by as high a margin as possible: a prize was given not (as in the previous experiment) to members of the winning team in each game, but to the members of the team which, out of all the teams in the condition, beat its opponents by the greatest margin.

The other five groups played a more cooperative game. ${ }^{1}$ In this game, all the four players in a group were on the same team, and the object was to score as highly as possible. Members of the team that got the highest score in the condition were awarded a prize. In neither condition of the game were players told how many other teams were playing or what score they had to beat.

The game played in this experiment was very similar to the game described in Chapter 5. It consisted of a series of fifteen timed rounds in each of which every player was, as in the previous experiment, paired pseudo-randomly with one of the other players. As before, a round of the game involved using an instantmessenger-style program to negotiate to exchange resources by typing messages in an "alien language" (which consisted this time of twenty randomly generated words; see Section 6.1.6), and, in the second stage, choosing whether or not to give anything away.

\footnotetext{
${ }^{1}$ This condition will be referred to as the "Cooperative condition", and the other condition as the "Competitive condition". The names refer to within-game competition/cooperation. Both conditions, of course, involved elements of cooperation and competition.
} 
In the Competitive condition, players were paired with their teammates in the first round of the game and the first of the practice rounds (of which there were three) and were told as much. They were not told who they would be paired with in any other round.

\subsubsection{Participants}

Forty participants were recruited via a student employment website. The advertisement stated that the experiment "involved playing a game with three other participants using an alien language". It was specified that participants must have a high level of English (so that they would be able to understand the instructions without difficulty), and must not have studied linguistics or have done an experiment for the same experimenter previously. As the experiment involved a lot of reading and typing, prospective applicants were asked not to apply if they suffered seriously from dyslexia or RSI. All but seven of the forty participants were native speakers of English, and they came from a wide variety of academic backgrounds. They ranged in age from 19 to 31. Participants were assigned to games on the basis of availability and to teams at random. Each participant was paid £8, and the members of the winning team in each condition were awarded $£ 10$ each.

\subsubsection{Experimental procedure}

The procedure was very similar to that of the previous experiment (see Section 5.2.3). The instructions were revised and separate instructions written for the Cooperative condition (see Appendix A).

After they had spent ten minutes learning the alien language, participants played three practice rounds, whose score did not count towards the game proper. In both the Competitive and the Cooperative conditions, players were paired with a different partner in each of these practice rounds. In the Cooperative condition, players were told as much. In the Competitive condition, players were told that the first person they were paired with was their teammate. They were told the same in the game proper. Players in the Cooperative condition (where everyone was on the same team) were told nothing about the first round of the game proper; no player in either condition was told anything about who they would be paired with in any 
106Chapter 6. Controlling for the pressure to mark identity: a second experiment

of the later rounds of the game. As there were more practice rounds (three rather than two) and more rounds in the game proper (15 rather than 14) than there had been in the first experiment, the practice rounds were shorter in this experiment. See Section 6.1.4 for more details.

After the practice rounds, the players were given a minute to relax and have a last look over their wordlists, as before. These were then taken away, and players began the 15 rounds of the game proper (as before, players were not told how many rounds to expect). As noted above, players were told in advance that they would be paired with their teammates for the first round. They were told nothing about the following rounds. The order of these rounds was randomised, but no player was paired with any other player for more than two rounds in a row. Every player was paired with every one of the other players for five rounds. Every game in the Cooperative condition was matched with a game in the Competitive condition, such that the same alien language was used for both, and the order in which players were paired was kept the same in both.

\subsubsection{Game structure}

Players began the game with more points than in the first experiment (28), and the distribution was more uneven (see Section 6.1.5 for more details), but the five kinds of resource remained the same. As before, players could see their available resources on the left of the screen throughout the game. In this experiment, the CHAT stage lasted for 180 seconds in the game proper and 120 seconds in the practice rounds. The EXCHANGE stage lasted 30 seconds in the main game, and 20 seconds in the practice rounds. Only in the Competitive Condition were players asked, "Were you talking to someone on your own team?" since the question would be meaningless in the Cooperative condition. The FEEDBACK stage, in which players were reminded what they had given their partner and told what they received, lasted 20 seconds in both the practice rounds and the main game. In the Competitive condition, as before, players were told if their partner had been on their team or not; no information of this sort was given in the Cooperative condition.

Since every player could be paired with one of three other players, and 14 (the number of rounds in the first experiment) is not cleanly divisible by three, each 
game in the second experiment lasted 15 rounds. After all rounds had finished, the teams' scores were revealed, and the penalty was applied as in the first experiment. In the Cooperative condition, a score was given for only one team, which was equal to the sum of all the players' resources.

\subsubsection{Resources and scoring}

Players began the game with 28 points each, divided between five resources: meat, grain, water, fruit, and fish. Every player began the game with fifteen points in one resource, seven points in one resource, three points each in two resources, and no points in the remaining resource. These quantities were allocated to specific resources at random, with the constraints that no two players in the same game had precisely the same distribution, and that the resource in which a given player had no points would be the resource in which their teammate had 15 points (therefore, no team in any game had fewer than six points between them in any resource). It will be noticed that the initial distribution of resources was more uneven than in the first experiment (see Section 5.2.5). This was mainly intended to make the Cooperative condition more interesting. Given that players in this condition did not have to do anything but exchange lots of resources with each other, the main interest of the game lay in coordinating resources to avoid unbalancing them. Players therefore started the game in a more unbalanced state than in the first experiment.

The penalty was applied just as in the first experiment and, as in that experiment, players could see how many of a given resource they had available throughout the game, but not how many resources their team had, until the end of the game, when the final team scores (or score) were revealed.

\subsubsection{Alien language}

The alien language was somewhat revised, and consisted of twenty, rather than nineteen words. Redundancy was both removed and added. A separate word for "goodbye" was found to be unnecessary, as it had been very little used in the first experiment. However, separate words were provided for "need" and "want", and for "grain" and "corn". This was intended as a seed for variation along which teams might divide (see Section 6.3.2.1). 
108Chapter 6. Controlling for the pressure to mark identity: a second experiment

The following is a sample set of words:

$\begin{array}{ll}\text { hello/goodbye - suve } & \text { need - petupu } \\ \text { please }- \text { jimujo } & \text { give }- \text { hiwo } \\ \text { thanks - yiyife } & \text { meat - jewa } \\ \text { yes - tiligo } & \text { grain - fekeda } \\ \text { no/not - gefa } & \text { corn - kemohu } \\ \text { and - kuta } & \text { water - fibi } \\ \text { I - holaki } & \text { fruit - kena } \\ \text { you - doso } & \text { fish - payawu } \\ \text { have - gajopo } & \text { much/a lot of - zeyu } \\ \text { want - leli } & \text { question word - bazeki }\end{array}$

\subsubsection{Summary of differences between first and second experiments}

Several differences have been mentioned between this experiment and the one described in Chapter 5. Table 6.1 provides a summary of the most important ones.

\begin{tabular}{lll}
\hline & First experiment & Second experiment \\
\hline No. participants & 20 & 40 \\
No. conditions & 1 (Competitive) & 2 (Competitive, Cooperative) \\
No. rounds & 14 & 15 \\
No. practice rounds & 2 & 3 \\
Length of round (seconds) & 230 & 230 \\
Length of CHAT stage & 180 & 180 \\
Length of EXCHANGE stage & 30 & 30 \\
Length of FEEDBACK stage & 20 & 20 \\
Length of practice round & 260 & 160 \\
Initial points per player & 7 & 28 \\
Size of alien language & 19 words & 20 words \\
No. practice rounds & & \\
with teammate & $2 / 2$ & $1 / 3$ \\
No. rounds with teammate & & \\
(game proper) & $7 / 14$ & $5 / 15$ \\
\hline
\end{tabular}

Table 6.1: Summary of differences between first and second experiments 
Chapter 6. Controlling for the pressure to mark identity: a second experiment 109

\subsection{Summary of terms}

Most of the terms defined in Section 5.3 will not be used differently in this chapter, and so will not be redefined. One term (TEAM) is used with a slightly expanded meaning, however, and this is explained below, along with any new terms.

Competitive condition In this condition, PLAYERS were divided into two TEAMS of two and the object was for one's own team to be the opposing team by as great a margin as possible.

Cooperative condition In this condition, all four PLAYERS were considered to be on the same TEAM, and the object of the game was for this team to get as high a score as possible.

Pseudo-opponent See PSEUdo-TEAM.

Pseudo-team In the analysis of the COMPETITIVe CONDITION, a PlAYER's linguistic output is compared with that of their TEAMMATE and with that of their two opponents. So that players in the COOPERATIVE CONDITION can be compared on the same basis, each player in this condition is allotted a pseudo-teammate. This simply means the first player they are paired with. ${ }^{2}$ The other two players are regarded as PSEUDO-OPPONENTS.

Pseudo-teammate See PSEUdo-TEAM.

Team This means something slightly different depending on the condition: in every GAME of the COMPETITIVE CONDITION there were two teams of two; in the COOPERATIVE CONDITION there was one team of four.

\subsection{Results}

Tables 6.2 and 6.3 summarise the results for this experiment. As in the first experiment, there were two main dependent variables in this experiment:

1. Players' success in distinguishing between teammates and opponents (in the Competitive condition only).

2. The degree to which the alien language diverged into two "dialects".

\footnotetext{
${ }^{2}$ In the third experiment, described in Chapter 7 , it will take on a greater significance, as players in the third experiment were paired more often with their teammates or pseudo-teammates than with either of the other players (cf. the first experiment).
} 
110Chapter 6. Controlling for the pressure to mark identity: a second experiment

As will be discussed in more detail in Section 6.3.1, players did not do especially well at identifying their teammates, and there was no significant divergence in the alien language in either condition (see Section 6.3.2). Section 6.5 will discuss what this means. Section 6.3.3 will look at how teams' scores relate to other results, and Section 6.4 will discuss the strategies the players employed.

\begin{tabular}{|c|c|c|c|c|c|}
\hline & $\begin{array}{l}\text { Final } \\
\text { score }^{a}\end{array}$ & $\begin{array}{l}\text { Convergence } \\
\text { level }^{\text {b }}\end{array}$ & $\begin{array}{l}\% \text { Correct } \\
\text { recognitions }\end{array}$ & $\begin{array}{l}\text { Mean } \\
\text { gift }^{d}\end{array}$ & $\begin{array}{l}\text { Strategies } \\
\text { employed }^{\mathrm{e}}\end{array}$ \\
\hline \multicolumn{6}{|c|}{ Game 1} \\
\hline Team a & $110(126)$ & 0.89 & $61.5(26)$ & $2.3(4.1)$ & DR, II \\
\hline Team b & 102 (102) & 0.86 & $64.3(28)$ & $1.8(2.7)$ & DR, II \\
\hline \multicolumn{6}{|c|}{ Game 2} \\
\hline Team a & 114 (163) & 0.87 & $57.7(26)$ & $2.4(4.0)$ & DR \\
\hline Team b & $114(131)$ & 0.86 & $78.6(28)$ & 3.7 (6.2) & DR, M, II \\
\hline \multicolumn{6}{|l|}{ Game 3} \\
\hline Team a & $205(467)$ & 0.88 & $62.5(24)$ & 13.3 (23.2) & $\mathrm{DR}, \mathrm{M}$ \\
\hline Team b & $236(544)$ & 0.93 & $50(24)$ & 16.7 (32.7) & $\mathrm{DR}, \mathrm{II}$ \\
\hline \multicolumn{6}{|c|}{ Game 4} \\
\hline Team a & 129 (224) & 0.83 & $75(24)$ & $7.3(9.1)$ & $\mathrm{DR}$ \\
\hline Team b & $78(245)$ & 0.82 & $56(25)$ & $4.6(3.7)$ & DR \\
\hline \multicolumn{6}{|c|}{ Game 5} \\
\hline Team a & 131 (189) & 0.89 & 74.1 (27) & $5.2(6.1)$ & DR, II \\
\hline Team b & 110 (207) & 0.86 & $65.4(26)$ & $4.2(4.3)$ & $\mathrm{DR}, \mathrm{M}$ \\
\hline
\end{tabular}

Table 6.2: Summary of results from second experiment (Competitive condition).

${ }^{\text {a }}$ Provisional score given in brackets;

b 1 - mean distance between teammates (higher figure means greater convergence); see Section 6.3.2;

c Excludes first round and rounds where players made no response; number of responses made given in brackets (out of a possible 28);

d Based on all rounds; mean gift to teammates only given in brackets;

e See Section 6.4.1; DR = Dialect Recognition; $\mathrm{M}=$ Mimicry; II = Intentional Innovation; $\mathrm{SH}=$ Secret Handshake.

\subsubsection{Recognising team-mates}

\subsubsection{Feedback questionnaires}

Compared with the first experiment (see Section 5.4.1), there was much greater variation in players' reported abilities at distinguishing between teammates and 
Chapter 6. Controlling for the pressure to mark identity: a second experiment 111

\begin{tabular}{|c|c|c|c|}
\hline & Final score $^{a}$ & Convergence level $^{\mathrm{b}}$ & Mean gift \\
\hline \multicolumn{4}{|l|}{ Game 1} \\
\hline Team $\mathrm{a}^{\mathrm{d}}$ & $490(594)$ & 0.92 & $15.3(15.9)$ \\
\hline Team b & $401(460)$ & 0.87 & $16.1(14.2)$ \\
\hline Combined & 911 (1054) & & $15.7(15.1)$ \\
\hline \multicolumn{4}{|l|}{ Game 2} \\
\hline Team a & 197 (214) & 0.79 & $8.5(8.5)$ \\
\hline Team b & $296(329)$ & 0.88 & $5.8(5.3)$ \\
\hline Combined & $493(543)$ & & $7.15(6.9)$ \\
\hline \multicolumn{4}{|l|}{ Game 3} \\
\hline Team a & $128(140)$ & 0.82 & 4.1 (3.1) \\
\hline Team b & 187 (213) & 0.89 & $3.97(4.7)$ \\
\hline Combined & 323 (353) & & $4(3.9)$ \\
\hline \multicolumn{4}{|l|}{ Game 4} \\
\hline Team a & $156(174)$ & 0.89 & $5.7(6.1)$ \\
\hline Team b & $169(235)$ & 0.89 & $4.2(4.1)$ \\
\hline Combined & $325(409)$ & & $4.95(5.1)$ \\
\hline \multicolumn{4}{|l|}{ Game 5} \\
\hline Team a & $66(117)$ & 0.90 & $5.9(5.5)$ \\
\hline Team b & $253(279)$ & 0.87 & $3.6(4.3)$ \\
\hline Combined & $321(396)$ & & $4.8(4.9)$ \\
\hline
\end{tabular}

Table 6.3: Summary of results from second experiment (Cooperative condition).

a Provisional score given in brackets;

b 1 - mean distance between pseudo-teammates (higher figure means greater convergence); see Section 6.3.2;

c Based on all rounds; mean gift to pseudo-teammates only given in brackets;

d Refers to pseudo-teams, for comparison with Competitive condition (see Section 6.2). 
opponents. This time, as well as being asked, "Were you able to tell if you were chatting to someone on the same team as you?" players were also asked "Were you able to tell if you were chatting to someone on the other team?" As in the first experiment, they were also asked, "If so, how?"

Of the twenty participants in the Competitive condition, only ten claimed to have been able to recognise their teammates at least some of the time (of whom only three gave an unqualified "Yes"). Of the remaining ten, six responded with an unqualified "No", and two with, "Not really," or, "Not easily". Of the remaining two, one said he had tried to pay attention to what resources his teammate claimed to have, but found this had been misleading. The other said that she had initially identified a particular "misspelling" her teammate used, but that this had been corrected later on in the game. One player, intriguingly, claimed to have been able to tell that his teammate was female, but could give no explanation for this beyond "a gut feeling". In answer to the second question, about identifying members of the other team, fourteen said they had managed to do so at least some of the time (seven gave an unqualified "Yes"); of the remaining six, one said, "Not really," and the others gave an unqualified "No". Of the twenty-five answers to either question that included some information about what they relied on or tried to rely on, nineteen referred to linguistic cues, of which six responses mentioned greetings specifically. One other mentioned punctuation, and one mentioned that her teammate simply spoke less than her opponents. Of the remaining six responses, one said that an opponent had demonstrated "superior knowledge", but did not explain this; presumably, however, it refers to grasp of the language. One participant claimed, in answer to both questions, to have relied on "intuition" and to have started to "recognise a pattern". The others referred to tracking their teammates' resources, and one who succeeded in identifying their teammate on the basis of their greeting identified opponents by a "process of elimination".

Obviously, players in the Cooperative condition were not asked either of the above questions, since they were all supposed to be on the same team. They were, however, asked, "Did you start to recognise other players by their use of the alien language? If so, how?" Of the twenty players in this condition, only one answered no to this question. Fifteen said they had, two said they had to some extent, one 
Chapter 6. Controlling for the pressure to mark identity: a second experiment 113

was uncertain, and one mentioned that they had always managed to communicate especially successfully with one other player.

\subsubsection{Explicit and implicit judgements in the Competitive condition}

As can be seen from the third column of Table 6.2, more players in this experiment failed to answer the question posed in the second stage of each round: "Were you talking to a member of your own team?" In fact, only two of the ten teams had perfect records (compared with six in the first experiment; see Table 6.2), and 7.9\% of potential responses are missing (22 out of 280). It is not entirely clear what the reason was for this, though two partial explanations present themselves. First, as discussed in Section 6.3.1.1, players found it difficult in this game to identify their teammates, so a non-response may, in some cases, simply mean, "No idea". A second fact worth noting is that the two worst teams at responding (both in Game 3) also have the highest mean gift; six non-responses occurred in rounds 11-15, in which players had begun giving away large amounts of resources (see Section 6.4.2), and were therefore preoccupied in the second stage of these rounds with doing this, rather than answering the question.

All 258 judgements that were made were submitted to a binomial sign test. Assuming that players had a 0.5 chance of being right by guessing on a given round, the result is significant $(S=91$ incorrect; $p<.001)$. This, however, is rather less impressive than it seems. Had they guessed 1 or 2 every time, they would actually have done better: $S=80$ incorrect out of 280; assuming a 0.5 chance of success, as for the previous result, this would also be significant $(p<.001)$. The point, of course, is that this was not a straightforward 50-50 choice, as the players had a 2 in 3 chance of being right by guessing no. At best, therefore, players seem simply to have recognised that they were more likely to be paired with an opponent than a teammate, and this would have become increasingly obvious throughout the game. Definite responses (a guess of 1 or 4) were, as in the first experiment, more often right than wrong, but less impressively so (72 correct against 25 incorrect). There were also fewer of them (97 compared with 111 in the first experiment), in spite of the extra round. 
As discussed in Section 5.4.1, there is another measure that can be employed to get at players' judgements: gift size. As in the first experiment, players' gifts - as a proportion of the the player's resources at the time of donation-were analysed for those rounds where they voted 1 or 2 (that is, for rounds when they claimed to believe they were talking to opponents). Did players give more to teammates on these rounds than to opponents? In fact, a Wilcoxon $T$ test (where $N_{1}=32$ and $N_{2}$ $=121$ ) showed that gifts to team-mates were significantly larger in these rounds than gifts to opponents $(T=2504, p<.05)$; furthermore, gifts to team-mates in these rounds did not differ significantly from gifts to team-mates where players guessed 3 or $4\left(T=913.5, N_{1}=32\right.$ and $\left.N_{2}=61 ; p>.05\right)$. Though apparently rather mysterious, this suggests that, while players learnt that they were more likely to be paired with an opponent than a team-mate, and made their explicit judgements on this basis, they were still in many cases able to identify their team-mates, if only on a subconscious level. ${ }^{3}$

In this context, it should be noted that there was a greater tendency in this experiment towards "malign generosity" - players' attempting to sabotage their opponents by offloading unbalanced resources on them and giving them things they explicitly did not ask for (more details will be given in Section 6.4.2). This does not, of course, explain why gifts to teammates were larger than gifts to opponents.

\subsubsection{Chatting time}

The CHAT stage of every round lasted 180 seconds. However, players were not obliged to chat for the whole of the time. It therefore seemed likely that players might chat for longer in the Competitive condition than in the Cooperative condition. Since this interaction was the primary means of distinguishing between teammate and opponent, they might be expected to spend longer over it when such distinctions mattered. Intriguingly, however, players spent longer chatting in the Cooperative condition (mean: 159.67 seconds) than in the Competitive condition (mean: 153.58 seconds). A one-tailed T-test, performed on log-transformed data, showed this to be significant $(t=-2.23, d f=283.36, p<0.01)$. This may reflect players' overall difficulty with identifying teammates in the Competitive condition.

\footnotetext{
${ }^{3}$ To look at it another way: players found it difficult to trust their intuition when it came to making an explicit judgement.
} 
Not only did players have little confidence in their ability to identify teammateswhich would explain why they did not talk more in the Competitive than in the Cooperative condition - they also tended to assume they were talking to opponents (see Section 6.3.1.2). This would explain why they talked significantly less. In the Cooperative condition, the content of the conversation mattered, as players wanted to exchange useful resources with their teammates. In the Competitive condition, players did not generally want to help the person they thought they were talking to, so there was less point in exchanging information.

\subsubsection{Divergence}

In this experiment, there were 100 potential linguistic variables in each condition (20 initial words $\times 5$ games). In the Competitive condition, 68 of these had more than one variant to them by the end of the game, and 75 in the Cooperative condition. 289 new variants were introduced in the Competitive condition (giving a mean of 5.25 variants in every variable set), and 375 in the Cooperative condition (a mean of 6 in every set).

The same method was used to measure divergence in the alien language as was used in the first experiment (see Section 5.4.2 and Appendix C for more details). There was no significant divergence (Competitive condition: $p=.566$; Cooperative condition: $p=.937)$. The competitive and cooperative conditions were also compared directly by performing a Monte Carlo test on a vector of the scores for all variable sets; there was no significant difference between them $(p=.275)$. As in the first experiment, within-team convergence scores were compared for every game in the Competitive condition: there was no significant between-team difference in convergence levels in any game.

\subsubsection{Other sources of divergence}

As in the case of word-level variants, and as in the first experiment, there was no significant syntactic divergence in either condition (Competitive: $p=.18$; Cooperative: $p=.73)$ or any significant difference between conditions $(p=.195)$.

It was mentioned in Section 6.1.6 that players were given synonyms in the data: words for both "grain" and "corn", though there was only one resource to which 
either could correspond, and words for both "need" and "want", though there was little to distinguish the two in terms of the game. It was anticipated that this might provide a seed for divergence; it did not. Instead, the pattern was of all players, by the end of every game, settling on one of the two variables - a result that recalls Garrod and Doherty's (1994) findings. If the frequencies of winning variables in each game are summed and compared with the frequency of losing variables, then the ratio for the grain/corn pair was 284 to 24 in the Competitive condition and 361 to 31 in the Cooperative condition - one variable was used $92 \%$ of the time in both conditions, in other words. The ratios for the want/need pair were similar, if slightly less unequal: 705 to 124 (85\% dominance) in the Competitive condition; 607 to $219(73 \%)$ in the Cooperative condition.

\subsubsection{Comparison with score}

In the first experiment, the teams with the best recognition rates tended to get the best provisional score. In the second experiment, this did not occur. In fact, the team that made the most correct guesses in this condition had the lower score before penalty in every case (the same is true even if only definite guesses are counted)! This suggests, as is supported by players' own evaluations in feedback questionnaires, that players were not doing well at all at recognising their teammates, at least on a conscious level (see Section 6.3.1). Nor was there a significant correlation between level of convergence and score: $r(8)=.49, p>.05$ (the same was true of the Cooperative condition: $r(8)=.37, p>.05$ ), or a significant difference between levels of within-team convergence in any game.

\subsection{Strategy}

\subsubsection{Identification strategies}

Only three identification strategies were used in this condition:

- Dialect Recognition (DR): try to remember how different players use the language and identify them based on this.

- Intentional Innovation (II): make changes to the language that are likely to be recognisable. 
- Mimicry (M): try to sound as much like the person you're talking to as possible.

It was suggested in Section 5.5 that there was some evidence for implicational universals of strategy. The evidence is poorer here. Certainly, all players claimed to have used Dialect Recognition, but there was no sign that either Intentional Innovation or Mimicry was more basic than the other, as can be seen in Table 6.2. There were, furthermore, no clear cases of a Secret Handshake (see Section 5.5.1), although there were two cases of Intentional Innovation in greetings, which could plausibly have developed into a Handshake. A player in Team 1b, for example, started saying "hello" (loku) twice in a row (as separate utterances) from the tenth round onwards, which her teammate noticed. This does not count as a Secret Handshake, however, as there was no specific response required. Similarly, a player in Team 3b started repeating the word for "hello" three times, in one utterance, at the start of a round (suve suve suve). However, her teammate failed to catch on, and she gave up the endeavour. It is worth noting, however, that this player was also the least conservative player in the condition (she gave away a mean of 16.9 points per round; see Section 6.4.2), and the only player in the condition to give her opponents less than half what she gave her team-mate. Her team won the game and was the highest scoring team in the condition.

\subsubsection{Giving behaviour}

The fourth column of Table 6.2 and the third column of Table 6.3 show the mean amount of resources given away per round by each team and pseudo-team, and the mean amount given to (pseudo-)teammates (whether or not players guessed who they were paired with). As before, every game in the Competitive condition for which there was a winner (Game 2 was a draw) was won by the most generous team, and combined mean gift is clearly correlated with score $(r(8)=.977, p<.0005) .{ }^{4}$ In the Cooperative condition, the combined mean gift also correlates with score $(r(3)=.99997, p<.0005)$, although this is rather trivial, as every gift in this condition contributes to the shared wealth. In every game in this condition, the

\footnotetext{
${ }^{4} \mathrm{As}$ for the previous experiment, this is based on the provisional score, as the most meaningful measure for these purposes. The correlation is still highly significant for final score as well, however.
} 
pseudo-team with the largest mean gift always has a smaller share of the score at the end of the game. This too is not in the least surprising, since players in this condition were not trying to keep their resources from anyone.

As mentioned in Section 6.3.1.2, players in this experiment were more inclined than in the first experiment to malign generosity. Eleven players claimed in feedback questionnaires to have employed this strategy, and two more to have used it as an occasional alternative to giving away nothing. This most likely reflects the structure of the game. Players were paired mostly with opponents, and found it difficult to identify teammates; this meant that it was difficult to actively help their teammates. The only remaining strategy was to try to actively scupper their opponents.

Overall, most players were rather conservative in their gift-giving. No player in the Competitive condition gave away more than half their resources, even in the first round of the game, when they knew they were paired with their teammates. Even in the Cooperative condition, players were cautious. Game 1 in this condition is something of an exception, and here one player did give away everything in the first round, and continued to be generous throughout. This game contained the only teams in the condition who gave away a mean gift of over 10; there were only two such teams in the Competitive condition too, both in Game 3. Figure 6.1 shows the pattern of gift giving over the course of the game. For most of the game, players were rather conservative. However, they began giving away large amounts of resources in the last five rounds of the game - a behaviour that appears to have originated with Player 4 in the eleventh round and spread to the others. Players did not discriminate successfully between team-mates and opponents in their generosity, however. What, then, explains this behaviour? Feedback questionnaires suggest players were mainly attempting to "dump" their excess points, with the intention of unbalancing their opponents' resources. There is an element of desperation in this, however, evidenced by the late stage at which this occurred, and Player 4's comment that "strategy went out the window towards the end of the game."

\subsubsection{Summary of results}

In this experiment, players did not do well at recognising their teammates (although they noticed how infrequently they were paired with them) and the alien 
Chapter 6. Controlling for the pressure to mark identity: a second experiment 119

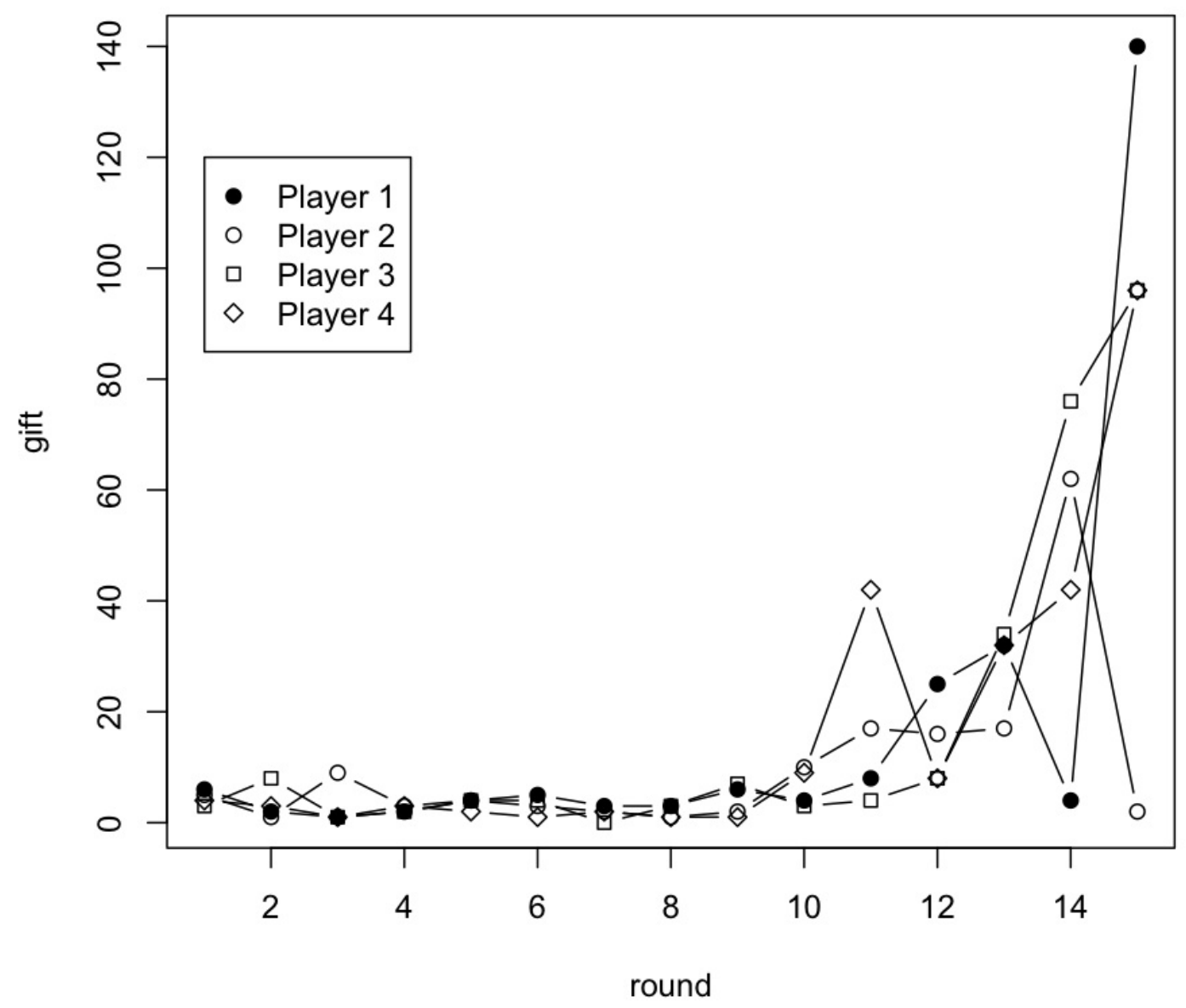

Figure 6.1: Gifts per round in Game 3 of the Competitive condition

language did not diverge into team dialects, as it did in the first experiment, in either condition.

Players in this experiment were very conservative in their gift giving, and there was a greater trend towards "malign generosity" (see Section 6.4.2) than in the first experiment. There were no examples of the most successful and salient strategy used in the first experiment - the Secret Handshake - presumably because players found the low level of contact with their teammates made it very hard to establish one. 
120Chapter 6. Controlling for the pressure to mark identity: a second experiment

\subsection{Discussion}

This experiment avoided most of the shortcomings discussed in Section 5.6: players were paired knowingly with their teammates far less; players did not interact more with any player than with the others; no participants had ever studied linguistics; finally, there was no power cut in this experiment. The problem of players neglecting to say whether they were talking to their teammate or not in the second stage of a round, however, remained. There was also one new shortcoming introduced in this experiment: a tendency, owing to the extra round, for the experiment to run slightly over time. This was partly due to the game simply being longer in this experiment than in the first, owing to the extra practice round and the extra round in the game proper. It was only three minutes longer, however. The whole session was made longer by the fact that participants were given as long as they wanted to read through the instructions, and some participants would take a relatively long time over this.

Neither of these problems was significant, however, and they are highly unlikely to have influenced the answers to the main research questions. These alone, in other words, were no reason to carry out a further experiment. There were other reasons for this. The results of this experiment are clearly somewhat discouraging for the social-selection model: they do not allow us to reject the null hypothesis that the seemingly exciting results from the first experiment are explicable purely with reference to frequency of interaction. This in itself, of course, is also no reason to run a further experiment - it might be the case, after all, that divergence is explicable purely with reference to frequency of interaction, and that finding would be an interesting one. The reason for a further experiment, which will be described in Chapter 7, is that the results from the second experiment also do not allow us to reject the hypothesis that social marking played some role in the first experiment. Had divergence occurred in the second experiment, the results would have been very interesting. Since it did not, all they seem to suggest is that frequency of interaction is important; they do not tell us how important, or if it is the only relevant factor in divergence. It was to try to get at this that a third experiment was carried out. 


\section{Chapter 7}

\section{Raising the frequency of interaction: a third exper- iment}

In the first experiment, described in Chapter 5, the alien language showed significant divergence into team dialects (see Section 5.4.2). Based on the data from this experiment alone, this could have been due either to the pressure to mark identity, or to the relatively high frequency of interaction between teammates $(50 \%$, while the other rounds were split between the two opponents). The second experiment, described in Chapter 6, lowered this frequency of interaction so that every player interacted an equal amount of time with every other player. It also introduced a control condition in which the frequency of interaction was kept the same, but there was no pressure to distinguish between teammates and opponents. In this experiment, the alien language did not diverge, and there was no significant difference between the Competitive and Cooperative conditions. This suggests that frequency of interaction does play a role, which should not be surprising. As noted in the introduction to Chapter 6, there must be some minimum level of interaction with one's teammate for sufficient accommodation between teammates to occur. However, it does not mean that pressure for social marking does not play any role at all. It may be that variation in frequency of interaction alone is sufficient for divergence, but it may also be that accommodation can be modulated by pressures for social marking. The next step is obvious: an experiment with the same frequency of interaction as in the first experiment, but incorporating the two conditions of the 
second experiment (along with other improvements introduced in that experiment). This chapter describes the results of doing precisely that.

\subsection{Description of experiment}

\subsubsection{Overview}

This experiment was carried out in October 2008, and involved forty participants. These participants were divided between a Competitive and a Cooperative condition just as in the second experiment, and the two conditions worked in the same way. This time, however, the game proper lasted for 16, rather than 15, rounds. As mentioned in Section 6.5, there had been a tendency for the experiment to run slightly over time. To avoid worsening this, the rounds were made slightly shorter (see Section 7.1.4). The number of practice rounds, the order in which players were paired in them, and the information given to players about the first round (that they would be paired with their teammate) were all kept the same as in the second experiment. Players were paid the same amount, and prizes were awarded on the same basis.

\subsubsection{Participants}

Forty participants were recruited via a student employment website. The advertisement stated that the experiment "involved playing a game with three other participants using an alien language". It was specified that participants must have a high level of English (so that they would be able to understand the instructions without difficulty), and must not have studied linguistics or have done an experiment for the same experimenter previously. As the experiment involved a lot of reading and typing, prospective applicants were asked not to apply if they suffered seriously from dyslexia or RSI. All but seven of the forty participants were native speakers of English, and they came from a wide variety of academic backgrounds. They ranged in age from 18 to 34. Participants were assigned to games on the basis of availability and to teams at random. Each participant was paid $£ 8$, and the members of the winning team in each condition were awarded $£ 10$ each. 


\subsubsection{Experimental procedure}

The procedure was very similar to that for the previous experiment (see Section 5.2.3). The instructions were again revised for clarity, as some participants had taken a relatively long time to read through them in the second experiment (see Section 6.5; instructions can be compared in Appendix A. There is further discussion in Section 8.1).

In this experiment, every player was paired with their teammate for 8 rounds and for 4 rounds each with their two opponents. This meant that there were 16 rounds in all in the game proper; these were preceded by 3 practice rounds, as in the second experiment. As detailed in Section 7.1.4, the rounds were slightly shorter in this experiment. The experimental design did not differ in any other respects.

\subsubsection{Game structure}

As in the second experiment, players began the game with 28 points, and these points were distributed in the same way. In this experiment, the CHAT stage lasted for 160 seconds in the game proper and 120 seconds in the practice rounds. The EXCHANGE stage lasted 30 seconds, and the FEEDBACK stage lasted 15 seconds, in both the main game and the practice rounds. There were 3 practice rounds and 16 rounds in the game proper.

\subsubsection{Resources and scoring}

Resources and scoring worked in the same way in this experiment as in the second experiment. See Section 6.1.5 for more information.

\subsubsection{Alien language}

The alien language had the same number of words and the same meanings as in the second experiment. The alien words themselves were generated anew for each pair of games; alien dictionaries from the second experiment were not reused. 


\subsubsection{Summary of differences between experiments}

A few differences have been mentioned between this experiment and the one described in Chapter 6. Table 7.1 provides a summary. The instructions were also revised for clarity (see Appendix A).

\begin{tabular}{llll}
\hline & First & Second & Third \\
\hline No. participants & 20 & 40 & 40 \\
No. conditions & 1 & 2 & 2 \\
No. rounds & 14 & 15 & 16 \\
No. practice rounds & 2 & 3 & 3 \\
Length of round (seconds) & 230 & 230 & 205 \\
Length of CHAT stage & 180 & 180 & 160 \\
Length of EXCHANGE stage & 30 & 30 & 30 \\
Length of FEEDBACK stage & 20 & 20 & 15 \\
Length of practice round & 260 & 160 & 165 \\
Initial points per player & 7 & 28 & 28 \\
Size of alien language & 19 words & 20 words & 20 words \\
No. practice rounds & & & \\
with teammate & $2 / 2$ & $1 / 3$ & $1 / 3$ \\
No. rounds with teammate & & & \\
(game proper) & $7 / 14$ & $5 / 15$ & $8 / 16$ \\
\hline
\end{tabular}

Table 7.1: Summary of differences between first, second, and third experiments

\section{$7.2 \quad$ Results}

Tables 7.2 and 7.3 summarise the results for this experiment. As in the first and second experiments, there were two main dependent variables:

1. Players' success in distinguishing between teammates and opponents (in the Competitive condition only).

2. The degree to which the alien language diverged into two "dialects".

As discussed in more detail in Section 7.2.1, players in the Competitive Condition succeeded in identifying their teammates. Moreover, the alien language diverged significantly in this condition, but not in the Cooperative condition (see Section 7.2.2). This suggests that social factors do play a role in language divergence. Section 7.4 will discuss this in more detail. Section 7.2 .3 will look at how teams' scores relate 
to other results, and Section 7.3 will discuss the players' strategies.

\begin{tabular}{|c|c|c|c|c|c|}
\hline & $\begin{array}{l}\text { Final } \\
\text { score }^{a}\end{array}$ & $\begin{array}{l}\text { Convergence } \\
\text { level }^{\text {b }}\end{array}$ & $\begin{array}{l}\% \text { Correct } \\
\text { recognitions }\end{array}$ & $\begin{array}{l}\text { Mean } \\
\text { gift }^{\mathrm{d}}\end{array}$ & $\begin{array}{l}\text { Strategies } \\
\text { employed }^{\mathrm{e}}\end{array}$ \\
\hline \multicolumn{6}{|l|}{ Game 1} \\
\hline Team a & $588(659)$ & 0.86 & $92.6(27)$ & $20.8(38.1)$ & $\mathrm{DR}, \mathrm{M}, \mathrm{II}, \mathrm{SH}$ \\
\hline Team b & $195(251)$ & 0.92 & $72.4(29)$ & $4.2(6.8)$ & $\mathrm{DR}, \mathrm{M}$ \\
\hline \multicolumn{6}{|l|}{ Game 2} \\
\hline Team a & $59(89)$ & 0.92 & $60(30)$ & $2.1(2.5)$ & DR, II \\
\hline Team b & $141(149)$ & 0.89 & $68.97(29)$ & $1.8(3.1)$ & DR, II \\
\hline \multicolumn{6}{|c|}{ Game 3} \\
\hline Team a & 146 (162) & 0.87 & $65.5(29)$ & $3.9(6.1)$ & DR, M, II \\
\hline Team b & $122(161)$ & 0.86 & $83.3(30)$ & $2.7(4.4)$ & $\mathrm{DR}, \mathrm{M}, \mathrm{II}$ \\
\hline \multicolumn{6}{|l|}{ Game 4} \\
\hline Team a & $142(176)$ & 0.93 & $62.96(27)$ & $4.3(6.1)$ & DR \\
\hline Team b & $1057(1248)$ & 0.86 & $100(25)$ & $36.8(71.6)$ & DR, II, SH \\
\hline \multicolumn{6}{|l|}{ Game 5} \\
\hline Team a & $78(91)$ & 0.88 & $62.1(29)$ & $1.2(1.7)$ & DR \\
\hline Team b & $95(95)$ & 0.86 & $73.3(30)$ & $1.1(1.6)$ & DR, II \\
\hline
\end{tabular}

Table 7.2: Summary of results from third experiment (Competitive condition).

a Provisional score given in brackets;

b 1 - mean distance between teammates (higher figure means greater convergence); see Section 7.2.2;

${ }^{\mathrm{c}}$ Excludes first two rounds and rounds where players made no response; number of responses made given in brackets (out of a possible 30);

${ }^{\mathrm{d}}$ Based on all rounds; mean gift to teammates only given in brackets;

e See Section 7.3.1; DR = Dialect Recognition; $\mathrm{M}=$ Mimicry; II = Intentional innovation; $\mathrm{SH}=$ Secret Handshake.

\subsubsection{Recognising team-mates}

\subsubsection{Feedback questionnaires}

As in the second experiment, players in the Competitive condition were asked if they had been able to tell if they were talking to their teammate, and if they had been able to tell if they had been talking to an opponent. Eight of the twenty participants gave an unqualified "Yes", citing linguistic cues (including greetings). Four said they had sometimes been successful, and four said they had become successful towards the end of the game. Only four players said no. In the Cooperative condition, in 


\begin{tabular}{|c|c|c|c|}
\hline & Final score ${ }^{\mathrm{a}}$ & Convergence level ${ }^{b}$ & Mean giftc \\
\hline \multicolumn{4}{|l|}{ Game 1} \\
\hline Team $\mathrm{a}^{\mathrm{d}}$ & 252 (309) & 0.90 & $5.8(6.9)$ \\
\hline Team b & 179 (194) & 0.85 & $6.4(6.1)$ \\
\hline Combined & $433(503)$ & & $6.1(6.5)$ \\
\hline \multicolumn{4}{|l|}{ Game 2} \\
\hline Team a & $212(370)$ & 0.89 & $15.4(15.94)$ \\
\hline Team b & $477(526)$ & 0.86 & $9.1(8.8)$ \\
\hline Combined & $691(896)$ & & $12.3(12.4)$ \\
\hline \multicolumn{4}{|l|}{ Game 3} \\
\hline Team a & 367 (387) & 0.85 & $14.5(15.9)$ \\
\hline Team b & $438(456)$ & 0.87 & $8.3(7.7)$ \\
\hline Combined & $817(843)$ & & $11.4(11.8)$ \\
\hline \multicolumn{4}{|l|}{ Game 4} \\
\hline Team a & 195 (232) & 0.80 & $6.9(6.6)$ \\
\hline Team b & $282(313)$ & 0.94 & $6.7(7.5)$ \\
\hline Combined & $477(545)$ & & $6.8(7.1)$ \\
\hline \multicolumn{4}{|l|}{ Game 5} \\
\hline Team a & $690(1421)$ & 0.89 & $24.97(26.3)$ \\
\hline Team b & $578(679)$ & 0.91 & 37.2 (33) \\
\hline Combined & 1411 (2100) & & 31.1 (29.7) \\
\hline
\end{tabular}

Table 7.3: Summary of results from third experiment (Cooperative condition).

a Provisional score given in brackets;

b 1 - mean distance between pseudo-teammates (higher figure means greater convergence); see Section 7.2.2;

c Based on all rounds; mean gift to pseudo-teammates only given in brackets;

${ }^{\mathrm{d}}$ Refers to pseudo-teams, for comparison with Competitive condition (see Section 6.2). 
answer to the question, "Did you start to recognise other players by their use of the alien language?" ten of the twenty players gave an unqualified "Yes", three said they had occasionally done so, four said they had not, but that they had been aware of differences between the other players' use of language. The other three gave an unqualified "No". ${ }^{1}$

Since a player in the second experiment had claimed to have been able to tell that his teammate was female, players in this experiment were also asked, "Could you tell anything about who your team-mate was (gender, age, identity etc.)? How?" Only four people made any guess. These are worth reproducing here if only for the window they provide onto gender stereotypes:

- (By a male participant) "Possibly female because I think guys are less prone to use exclamation marks." 2

- (By the female teammate of the previous participant) "I thought my partner might be female, with use of exclamations etc."

- (By a male participant) "I think a guy. He wasn't too generous. Just a guess..."

- (By a female participant) "Female. She seemed very nice!"

Similarly, players in the Cooperative condition were asked, "Could you ever tell anything (gender, age, identity etc.) about who you were talking to? How?" Seventeen of the twenty participants answered no. The remaining three responded:

- (By a female participant) "I guessed one was probably male. Not sure why."

- (By a female participant) "It seemed that the emphasis on having a lot by misspelling 'mujeju' was expressive in a 'feminine' manner."

- (By a male participant) "No, not at all. I had an argument with who I thought was a girl but I'm not sure".

\footnotetext{
${ }^{1}$ However, one of these thought that other players had been able to recognise her "Because I typed on the screen before", presumably meaning that she had started typing messages during the feedback stage of the previous round.

${ }^{2}$ Interestingly, in answer to the question, "Were you able to recognise your teammate?" his (female) teammate replied: "Yes. I use a lot of exclamation marks when I write \& found the other person did this too."
} 


\subsubsection{Explicit and implicit judgements in the Competitive condition}

There were slightly fewer failures in this experiment to answer the question, "Were you chatting to a member of your own team?" This time, $5 \%$ of potential responses were missing (15 of 300; compared with almost $8 \%$ in the second experiment), and three teams had perfect records. This is a small improvement on the second experiment, but not an enormous one. As in the second experiment (see Section 6.3.1.2), there seems to have been a connection with generous gift-giving, as can be seen in the case of Teams 1a and 4b, for example. Both these teams developed Secret Handshakes (see Sections 5.5.1 and 7.3.1), which helped create a relatively secure environment for generous gift-giving between teammates, meaning that the members of these teams were preoccupied in the second stage of the round with giving away resources to each other.

In this experiment, unlike the second one, players were equally likely to be paired with an opponent as with a teammate, and they did significantly better than chance at noticing which was the case $(S=75$ incorrect out of $285 ; p<.001)$. In further contrast to the second experiment, there was no significant difference between gifts to opponents and teammates on those rounds where players thought they were paired with opponents $\left(T=3620.5, N_{1}=49\right.$ and $\left.N_{2}=127 ; p>.05\right)$. There was no significant difference, however, between the time spent chatting in the Competitive condition (mean: 140.74 seconds) and in the Cooperative condition (mean: 137.6937; $t=1.43, d f=297.45, p>.05)$. As in previous experiments, the vast majority of definite guesses were correct (148 against 27 incorrect).

\subsubsection{Divergence}

In this experiment, as in the second, there were 100 potential variables in each condition. In the Competitive condition, 73 of these had more than one variant to them by the end of the game, and 79 in the Cooperative condition. 349 new variants were introduced in the Competitive condition (giving a mean of 5.78 variants in every variable set), and 336 in the Cooperative condition (a mean of 5.25 in every set). 
The same method was used to measure divergence in the alien language as was used in the first two experiments (see Section 5.4.2 and Appendix C for more details).

There was a significant between-team difference in within-team convergence in one game of the Competitive condition: Game $4(p=.035)$. This is interesting, since the less converged of the two teams in this game employed a Secret Handshake (see Section 7.3.1). This suggests that the Handshake helped create a secure environment for the team that used it, in which there was less pressure for convergence in other respects. The overall divergence results for this experiment are also very interesting. In the first experiment, the divergence could have been explained by frequency of interaction alone. In the second experiment, there was no divergence in either condition. In this third experiment, however, there was a significant tendency towards divergence in the Competitive condition $(p=.001)$, but not in the Cooperative condition $(p=.154)$; and this difference in significance reflected a significant difference between conditions $(p=.027$; on the need for such a test, see Gelman and Stern 2006). Frequent interaction alone was not, therefore, sufficient to cause divergence, although a comparison between the second experiment and this one (see Chapter 9) suggests that both factors must play a role.

\subsubsection{Other sources of divergence}

As in the first two experiments, there was no significant tendency for syntactic divergence in either condition, although the result for the Competitive condition approached significance (Competitive: $p=.058$; Cooperative: $p=.99$ ); there was, moreover, a significant difference between the level of syntactic divergence in the two conditions $(p<.05)$.

Results on synonym pairs ("grain" vs "corn" and "want vs need") were as in the second experiment (see Section 6.3.2.1). For the grain/corn pair, the overall ratio of winning variable to losing variable was 263 to 25 (91\%) in the Competitive condition and 304 to 87 (78\%) in the Cooperative condition. The want/need ratios were 654 to 129 (84\%) in the Competitive condition and 691 to 188 (79\%) in the Cooperative. 


\subsubsection{Comparison with score}

In four out of the five games in the Competitive condition, the team that made the greatest number of correct judgements also won the game; and in the one game where this was not the case - Game 3-the scores before penalty were extremely close (161 and 162). Similarly, the team that made the most definite guesses won Games 1, 4 and 5. Game 3, as noted, was very close. Game 2 was not. In this game, however, one member of the winning team made only one definite guess, which may reflect nothing more than a more cautious personality.

Unlike the first experiment, there was a significant correlation between recognition rate - the percentage of rounds in which players correctly judged whether they were talking to a teammate or opponent-and score $(r(8)=.86, p<.005)$. Figure 7.1 is a plot of the relationship. As in previous experiments, there was no significant correlation between level of convergence and score (Competitive condition: $r(8)=-.38, p>.05$; Cooperative condition: $r(8)=-.25, p>.05)$ and, as noted in Section 7.2.2, there was a significant difference between levels of withinteam convergence in only one game-Game 4-where the most closely converged team was in fact the losing team. As discussed above, this should not surprise us. The overall levels of within-team convergence and between-team divergence are better treated as by-products of adaptive strategies rather than as strategies themselves, and one of the strategies employed by the winning team in Game 4 made further accommodation (by which convergence comes about; see Section 9.4) between teammates unnecessary.

\subsection{Strategy}

\subsubsection{Identification strategies}

All four strategies used in the first experiment were used again here:

- Dialect Recognition (DR): try to remember how different players use the language and identify them based on this.

- Intentional Innovation (II): make changes to the language that are likely to be recognisable. 


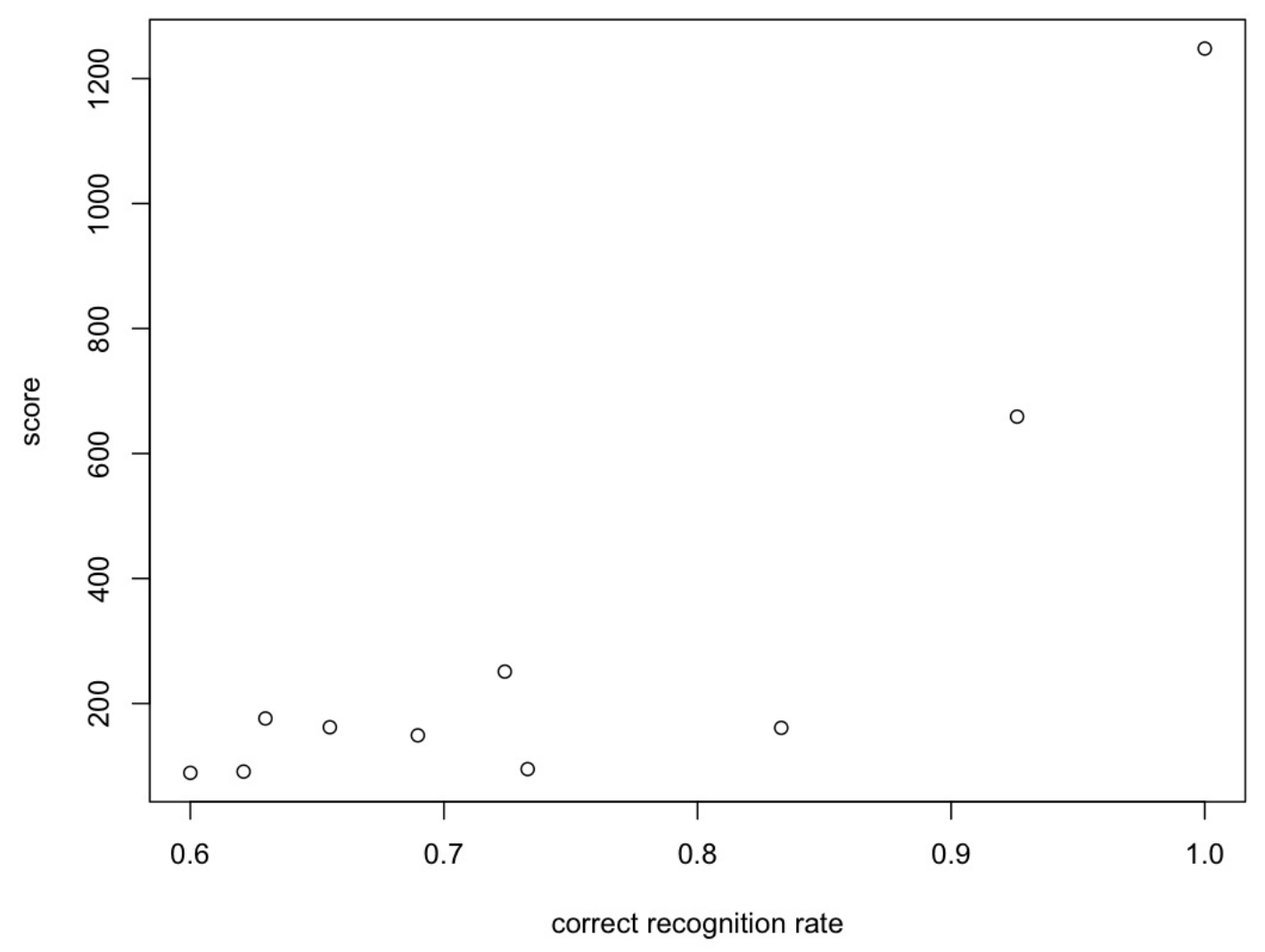

Figure 7.1: Scatterplot of recognition rate against score

- Mimicry (M): try to sound as much like the person you're talking to as possible.

- Secret Handshake (SH): say something at the beginning of the interaction that identifies you to your team-mate (and ideally not your opponent), and requires a specific response from them (see below for an example).

Again, inclusion of strategies in Table 7.2 is based mainly on self-reporting. As can be seen from the table, Dialect Recognition was again the only strategy to be used by all players, confirming that it is the simplest and most basic strategy. There is no reason, based on any of the data so far, to consider either Mimicry or Intentional Innovation as more basic than the other. Both are hard to identify in the data, and both may be underreported by the players themselves (with regard to mimicry, for example, players may mention trying to imitate their opponents to 
trick them, but may not mention trying to sound like their teammates). Assuming accurate reporting, however, this experiment produced the first case of Secret Handshake without Mimicry. This is not especially surprising. Both Intentional Innovation and Secret Handshake involve changing something in the language, and the latter implies the former. Mimicry, however, is unnecessary for the development of a Secret Handshake, although the first steps towards such a handshake may involve it.

Secret Handshakes were employed in this experiment by Teams 1a and 4b. The more sophisticated of the two Handshakes, and the most sophisticated to be used in any of the experiments was Team 1a's. It evolved to have three parts: one player gave the word for 'hello': vobu; the other responded by repeating it twice: vobu vobu; and the first responded again with vobu vobu!!!. This developed into a strict form. In the twelfth round, Player 3 tried to crack the code. The sequence went as follows:

Player 2: vobu

Player 3: vobu vobu

Player 2: vobu vobu!!!

Player 3: vobu vobu!!!

That final repetition of vobu vobu!!! was apparently enough to tell Player 2 that her partner for that round was not her teammate. The difficulty for an outsider in cracking such a code lies in never seeing the whole sequence in its entirety.

Team 4b's Handshake, although this team was more successful, was far simpler, and looks easy to crack. Player 4 introduced it in the very first of the practice rounds: she would greet everyone with Dulu X ("I [am] X'), while Player 3 would greet with Dulu A ("I [am] A"). This counts as a Secret Handshake, since it contains more than one element, and the first element to be used requires the other as a specific response. In fact, it is formally no less complex than the Handshake used in the first experiment (described in Section 5.5.1); it is merely more transparent. Compared with Team 1a's handshake, however, it is clearly less complex: the order or elements is freer and there are only two of them. The Handshake did not evolve further because the opposing team were apparently unable to crack it (although one member of Team $4 \mathrm{~b}$ reported fearing they would)! One of their opponents 
responded, both in one of the practice rounds and in four rounds of the game proper, with Dulu $k$. He apparently failed to understand the basis of what his opponents were doing, and was not recognised as their teammate. His own teammate made no similar attempts.

\subsubsection{Giving behaviour}

The fourth column of Table 7.2 and the third column of Table 7.3 show the mean amount of resources given away per round by each (pseudo-)team, and the mean amount given to (pseudo-)teammates (whether or not players guessed who they were paired with). In the Cooperative condition, there is the expected correlation between combined mean gift and score $(r(3)=.999996)$ and, as in the second experiment, the pseudo-team with the largest mean gift always has a smaller share of the score at the end of the game. In the Competitive condition, the team with the highest combined mean gift did not win the game in every case (although there is the expected correlation between mean gift and score: $r(8)=.996$ ); the team with the highest mean gift to teammates, however, did.

In both conditions, most players were again rather conservative in their giftgiving. In the first round of the Competitive condition, only one player gave away everything (in Team 4b), and only one other gave away more than half their resources (the other member of Team 4b). As in the second experiment, two teams stand out as being the least conservative, with a mean gift of over 10: Teams 1a and 4b. These teams are different from the two in the second experiment, however (see Section 6.4.2); first, they were not playing in the same game, so cannot have influenced each other; second, they were consistently generous - all gave away more than $20 \%$ of their resources on at least four rounds apart from the first (where all gave over $30 \%$ ). Third, they were more discriminating in who they gave their resources to. Finally, their generosity was benign, rather than malign; there is no reason to think they were deliberately trying to unbalance their opponents' resources. It is, of course, not surprising that generosity only occurs as a consistent behaviour in those teams that developed Secret Handshakes, for reasons already discussed.

Only one player claimed to have generally aimed to scupper his opponents by giving them large amounts of resources to unbalance their resources (cf. Sections 6.3.1 
and 6.4.2), although two others claimed to have done this to a small extent on occasion, but only when they had too much of something, and in addition to a general policy of giving nothing. Three players said they gave a little to opponents early in the game, but then started giving nothing. Two of these do not say why they gave anything at all in early rounds; the third, apparently misunderstanding the point of the game, or choosing to opt out of the competition, claimed he had been trying to "foster cooperation", but that he gave this up.

\subsubsection{Summary of results}

In this experiment, as in the first, players did considerably better than chance at identifying their teammates, and their behaviour in the game led the alien language to begin diverging significantly. This occurred only in the Competitive condition, however, where there was a pressure to distinguish between teammates and opponents. In the Cooperative condition, where there was no such pressure, but where frequency of interaction was similarly biased, the alien language did not diverge.

Players were once again (as in both previous experiments) notable for their conservatism in gift-giving in both conditions. All the main strategies used in the first experiment were seen again, although there were fewer examples of "malign generosity" in gift giving, whereby players attempt to sabotage their opponents by giving them large gifts that will unbalance their resources. This experiment saw the third example of a Secret Handshake, and the most complex example so far.

\subsection{Discussion}

The results of this experiment are highly intriguing. In Section 5.6, it was suggested that the divergence seen in the first experiment may simply be the result of frequency of interaction, or that the long period of knowing interaction between teammates at the start of the game might have played an important role. This third experiment, by having only one round of knowing interaction between teammates in the practice rounds and the game proper, ${ }^{3}$ and by including a control condition in which the only pressure for divergence is frequency of interaction, shows that this

\footnotetext{
${ }^{3}$ It would be interesting in future studies, however, to see whether players need any such rounds at all.
} 
cannot be the case. Nevertheless, as the results of the second experiment show, a pressure for social marking alone cannot explain the divergence. There must also be a minimum level of interaction between teammates (apparently around 50\%).

The second and third experiments can thus be treated as two conditions of one experiment, with the second experiment as a Low-frequency condition, and the third as a High-frequency condition. Figure 7.2 summarises the experimental design. Tables C.4 and C.5 compare divergence levels for all conditions.

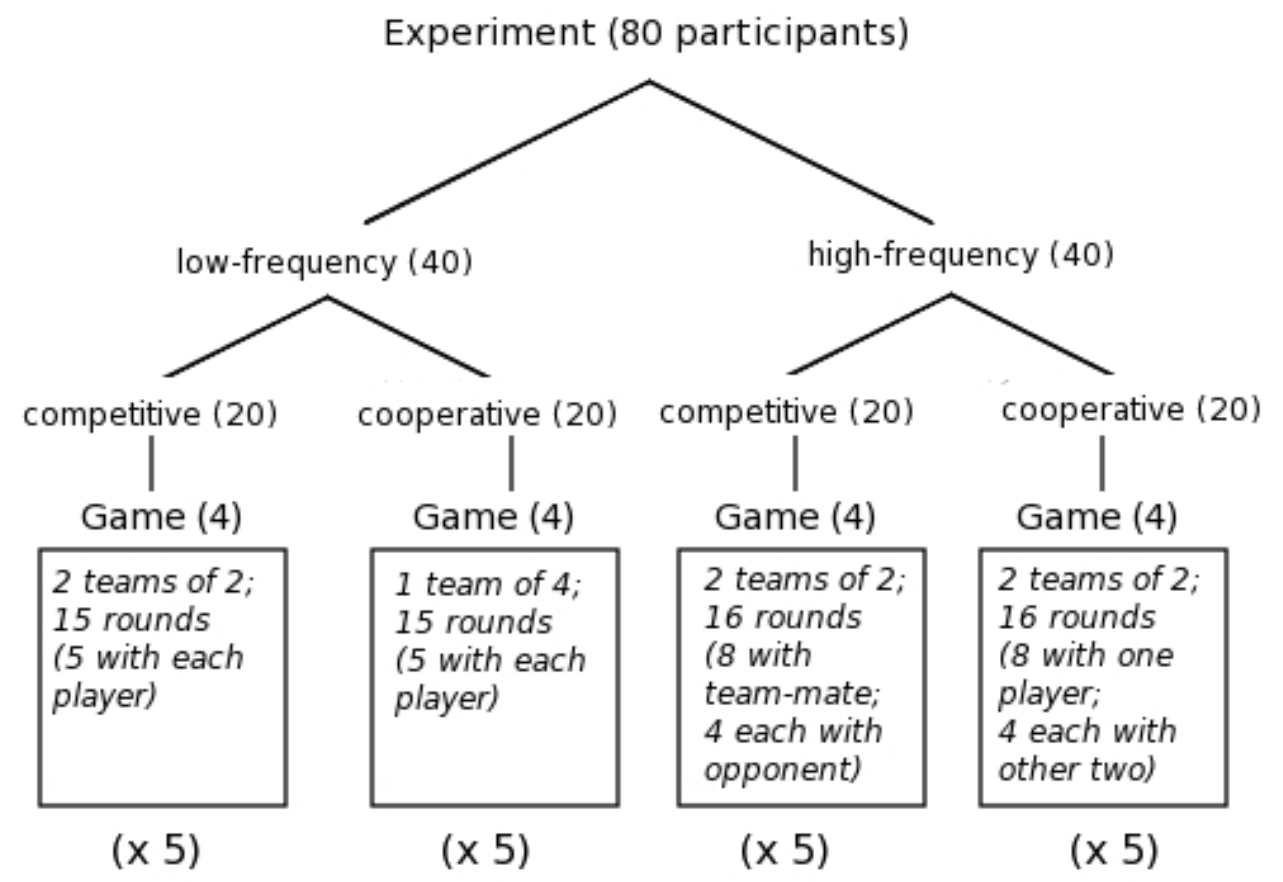

Figure 7.2: Diagram of experimental design (No. of participants given in brackets)

As can be seen Section 7.1.7, however, there were small differences between the two experiments beyond frequency of interaction. Chapter 8 will discuss these in more detail, and will describe a further small experiment designed to investigate the importance of these differences. 



\section{Chapter 8}

\section{Explaining variation in gift size: a fourth experi- ment}

\subsection{Comparing the second and third experiments}

Taken together, the results of the second and third experiments provide an intriguing picture, which supports and elaborates on the findings of the first experiment. The results suggest that a pressure to mark group identity, combined with at least a $50 \%$ level of contact between teammates, can sow the seeds of language divergence in a very short time, but that neither the social pressure nor the frequency of interaction alone is sufficient (although variation in frequency of interaction alone would doubtless lead to divergence over longer time periods; cf. Dunbar 2003, 230).

However, as summarised in Section 7.1.7, there are some differences aside from frequency of interaction between the second and third experiments. First, to fit more rounds into the third experiment, each round was made 25 seconds shorter (see Section 7.1.1 and 7.1.4). It is unlikely that this had any serious influence on the main research questions - the players' ability, that is, to distinguish between teammates and opponents, and the degree to which the alien language diverged into dialects. One would expect, after all, that having more time to chat would help players identify each other and pick up on linguistic cues, but players in the second experiment clearly found this a very hard task. The second change worth noting is that the instructions were revised for clarity, and this meant that the need to balance resources was less heavily emphasised in the instructions for the 
third experiment, although it was still stated more than once (see Appendix A to compare). Again, there is no reason to think that this had any influence on players' ability to recognise each other, or on the level of divergence in the alien language. However, it is possible that it might have encouraged greater conservativeness in gift-giving. While the difficulty in identifying teammates in the second experiment no doubt encouraged caution in the Competitive condition, one would not expect the same caution in the Cooperative condition, and there does seem to be a difference between the overall mean gift in the Cooperative condition of the second experiment (7.3) and of the third (13.5). Moreover, a large number of players in the second experiment claimed to have tried to sabotage their opponents by giving them large gifts so as to unbalance their resources (see Section 6.3.1.2). While this most likely reflects players' frustrations at being unable to recognise their teammates, leading them to focus on malign opponent-based strategies rather than benign teammatebased ones, it may also suggest that players in this experiment attached greater importance to balancing resources than in the third.

To confirm whether or not any of this had had an effect, it was decided to partially replicate the second experiment, using the instructions and the round length from the third experiment. To save time and money, only two games were played in each condition. In this chapter, and subsequent ones, for the sake of comparison, the second and fourth experiments will be referred to as the Low-frequency conditions, and the first and third experiments as the High-frequency conditions. ${ }^{1}$

\subsection{Description of experiment}

\subsubsection{Overview}

This experiment was carried out in January 2010, and involved sixteen participants. These participants were divided between a Competitive and a Cooperative condition just as in the second and third experiments, and the two conditions worked in the same way. The game proper lasted for 15 rounds, as in the second experiment, but each round lasted for 205 seconds, as in the third experiment. The number of

\footnotetext{
${ }^{1}$ These terms refer to the relative frequency with which a given player was paired with their teammate or pseudo-teammate.
} 
practice rounds, the order in which players were paired in them, and the information given to players about the first round (that they would be paired with their teammate) were all kept the same as in the second and third experiments. Owing to inflation, players were paid $£ 9$ rather than $£ 8$, but prizes were awarded as before.

\subsubsection{Participants}

Sixteen participants were recruited via a student employment website, and the same advertisement was used as for the second and third experiments. All but four of the sixteen participants were native speakers of English, and they came from a wide variety of academic backgrounds. They ranged in age from 18 to 25. Participants were assigned to games on the basis of availability and to teams at random. ${ }^{2}$ Each participant was paid $£ 9$, and the members of the winning team in each condition were awarded $£ 10$ each.

\subsubsection{Experimental procedure}

The procedure was very similar to that for the previous experiments (see Section 5.2.3), and the instructions were exactly as in the third experiment (see Appendix A).

As in the second experiment, every player was paired with their teammate for 5 rounds and also for 5 rounds each with their two opponents. This meant that there were 15 rounds in all in the game proper, preceded, as in the two previous experiments, by 3 practice rounds. Each round lasted 205 seconds.

\subsubsection{Game structure}

As in the second and third experiments, players began the game with 28 points, and these points were distributed in the same way. As in the third experiment, the CHAT stage lasted for 160 seconds in the game proper and 120 seconds in the practice rounds. The EXCHANGE stage lasted 30 seconds, and the FEEDBACK stage lasted 15 seconds, in both the main game and the practice rounds. There were 3 practice rounds and 15 rounds in the game proper.

\footnotetext{
${ }^{2}$ As in the first experiment, however, two participants in one game turned out unexpectedly to know each other; they were put, without their knowledge, in separate teams.
} 


\subsubsection{Resources and scoring}

Resources and scoring worked in the same way in this experiment as in the second and third experiments. See Section 6.1.5 for more information.

\subsubsection{Alien language}

The alien language had the same number of words and the same meanings as in the second and third experiments. As before, the alien words themselves were generated anew for each pair of games; alien dictionaries from the second and third experiments were not reused.

\subsubsection{Comparison of experiments}

Table 8.1 gives a full summary of differences between the four experiments.

\begin{tabular}{lllll}
\hline & First & Second & Third & Fourth \\
\hline No. participants & 20 & 40 & 40 & 16 \\
No. conditions & 1 & 2 & 2 & 2 \\
No. rounds & 14 & 15 & 16 & 15 \\
No. practice rounds & 2 & 3 & 3 & 3 \\
Length of round (seconds) & 230 & 230 & 205 & 205 \\
Length of CHAT stage & 180 & 180 & 160 & 160 \\
Length of EXCHANGE stage & 30 & 30 & 30 & 30 \\
Length of FEEDBACK stage & 20 & 20 & 15 & 15 \\
Length of practice round & 260 & 160 & 165 & 165 \\
Initial points per player & 7 & 28 & 28 & 28 \\
Size of alien language & 19 words & 20 words & 20 words & 20 words \\
No. practice rounds & & & & \\
$\quad$ with teammate & $2 / 2$ & $1 / 3$ & $1 / 3$ & $1 / 3$ \\
No. rounds with teammate & & & & \\
(game proper) & $7 / 14$ & $5 / 15$ & $8 / 16$ & $5 / 15$ \\
\hline
\end{tabular}

Table 8.1: Summary of differences between all four experiments

\subsection{Results}

Tables 8.2 and 8.3 summarise the results for this experiment. The main dependent variable for this experiment was the mean gift size, since it was expected that this might differ from that in the second experiment, owing to the emphasis put on 
keeping resources balanced in the instructions. In fact, the overall mean gift size was considerably higher in the Cooperative condition than in previous experiments, and slightly lower in the Competitive condition. Table 8.4 provides a summary. These results are somewhat surprising, and will be discussed in Section 8.3.1.

Other dependent variables will be discussed in Sections 8.3.2 to 8.4.1.

\begin{tabular}{|c|c|c|c|c|c|}
\hline & $\begin{array}{l}\text { Final } \\
\text { score }^{a}\end{array}$ & $\begin{array}{l}\text { Convergence } \\
\text { level }^{b}\end{array}$ & $\begin{array}{l}\% \text { Correct } \\
\text { recognitions }\end{array}$ & $\begin{array}{l}\text { Mean } \\
\text { gift }^{\mathrm{d}}\end{array}$ & $\begin{array}{l}\text { Strategies } \\
\text { employed }^{\mathrm{e}}\end{array}$ \\
\hline \multicolumn{6}{|l|}{ Game 1} \\
\hline Team a & 170 (199) & 0.92 & $80.8(26)$ & $6.5(11.4)$ & DR M II SH \\
\hline Team b & 94 (134) & 0.84 & 67.9 (28) & 2.1 (3.7) & DR M II \\
\hline \multicolumn{6}{|l|}{ Game 2} \\
\hline Team a & 178 (221) & 0.83 & $76.92(26)$ & $6.6(12)$ & DR M \\
\hline Team b & 169 (183) & 0.82 & $57.7(26)$ & $3.2(3.4)$ & $\mathrm{DR}$ \\
\hline
\end{tabular}

Table 8.2: Summary of results from fourth experiment (Competitive condition).

a Provisional score given in brackets;

b 1 - mean distance between teammates (higher figure means greater convergence); see Section 8.3.3;

${ }^{c}$ Excludes first two rounds and rounds where players made no response; number of responses made given in brackets (out of a possible 28);

${ }^{\mathrm{d}}$ Based on all rounds; mean gift to teammates only given in brackets;

e See Section 8.4.1; DR = Dialect Recognition; $\mathrm{M}=$ Mimicry; II = Intentional Innovation; $\mathrm{SH}=$ Secret Handshake.

\subsubsection{Giving behaviour}

The fourth column of Table 8.2 and the third column of Table 8.3 show the mean amount of resources given away per round by each team of pseudo-team, and the mean amount given to (pseudo-)teammates (whether or not players guessed who they were paired with). Only one player in the Competitive condition claimed to have attempted to sabotage his opponents by giving them a lot of resources; the other seven tried to give nothing. In the Cooperative condition, there is the expected relationship between combined mean gift and score. In both games in this condition, as before, the pseudo-team with the largest mean gift has the smaller share of the score at the end of the game. In both games in the Competitive 


\begin{tabular}{lccl}
\hline & Final score $^{\text {a }}$ & Convergence level $^{\text {b }}$ & Mean gift \\
\hline Game 1 & & & \\
Team a & $1648($ 2927) & 0.84 & $61.8(56.3)$ \\
Team b & $1026(1661)$ & 0.87 & $87.4(82.1)$ \\
Combined & $2675(4588)$ & & $74.6(69.2)$ \\
Game 2 & & & \\
Team a & $2956(3601)$ & 0.93 & $75.1(71)$ \\
Team b & $1909(2039)$ & 0.82 & $109.2(108.6)$ \\
Combined & $5080(5640)$ & & $92.2(89.8)$ \\
\hline
\end{tabular}

Table 8.3: Summary of results from fourth experiment (Cooperative condition).

${ }^{a}$ Provisional score given in brackets;

b 1 - mean distance between pseudo-teammates (higher figure means greater convergence); see Section 8.3.3;

${ }^{\mathrm{c}}$ Based on all rounds; mean gift to pseudo-teammates only given in brackets;

${ }^{\mathrm{d}}$ Refers to pseudo-teams, for comparison with Competitive condition (see Section 6.2).

condition, the team with the highest mean gift won the game. In both conditions, however, the data points are too few for inferential statistics to be very meaningful.

Table 8.4 summarises the mean gifts in the second, third, and fourth experiments. There are some surprises, most notably that the overall mean gift size for the Cooperative condition is considerably higher in the fourth experiment than in either of the previous experiments, and for the Competitive condition, rather lower; the same is true of the mean gift to teammates. It is far from immediately clear why this should be.

\begin{tabular}{llll}
\hline & $\begin{array}{l}\text { Second } \\
\text { experiment }\end{array}$ & $\begin{array}{l}\text { Third } \\
\text { experiment }\end{array}$ & $\begin{array}{l}\text { Fourth } \\
\text { experiment }\end{array}$ \\
\hline Competitive (overall) & 6.2 & 7.9 & 4.6 \\
Competitive (teammate) & 9.6 & 14.2 & 7.6 \\
Cooperative & 7.3 & 13.5 & 83.4 \\
\hline
\end{tabular}

Table 8.4: Summary of mean gifts

There are several factors that might be expected to influence gift size:

1. Identity of recipient. 
2. Relative certainty about identifying teammates.

3. Perceived importance of balancing resources.

4. Individual variation.

The first seems to make a clear difference. The overall mean gift to teammates in the Competitive condition is greater in every experiment than the overall mean gift; the same difference is not seen in Cooperative conditions. This is as should be expected, and means that most players at least understand the aim of the game. We should expect the second factor to amplify this; the more certain players are in their identification of teammates, the larger we should expect their gifts to be. This may go some way to account for the difference between the second and third experiments with respect to the Competitive condition. Based on this, we should expect players to behave in every round of the Cooperative conditions as they do in Competitive rounds where they are certain they are paired with teammates: these two factors should encourage large gifts. The third factor may pull in the other direction, however. It is easier, if one is concerned about dramatically unbalancing resources, to be relatively conservative about gift giving. On the other hand, while most participants reported trying to give as little as possible to opponents, a few claimed, on occasion, to have deliberately tried to unbalance their opponents' resources by giving them a lot of some resource that they already claimed to have too much of (see Sections 5.5.2, 6.4.2, and 7.3.2, where this is referred to as "malign generosity"). Very few participants seem to have done this, however, as evidenced both by their own feedback and by the fact that the mean gifts to teammates were higher than to opponents. In any case, as suggested above in Section 8.1, it seems plausible that the greater emphasis on balancing resources in the instructions for the second experiment explains the difference between that experiment and the third with respect to mean gifts in the Cooperative condition.

This leaves the results from the fourth experiment. The above will not account for the differences between these results and the previous experiments. The Competitive conditions are not such a problem to explain. While the overall mean is smaller than in the second experiment, this can be explained by the unusual behaviour of players in Game 3 of that experiment (see Section 6.4.2). If data from this game is excluded, the mean gift becomes 3.9 overall, and 5 to teammates. The 
results from the fourth experiment then look as expected: slightly higher than in the second experiment, perhaps owing to less caution over balancing resources, but somewhat lower than in the third experiment, owing to greater uncertainty as to the identity of teammates.

The mean gifts from the Cooperative conditions, however, cannot be explained so easily. The question is perhaps not so much why gifts in the fourth experiment should be so high, but why they should be so low in the other experiments. Caution about balancing resources may go some way to explaining it in the second experiment, but there is no reason for any greater caution in the third than in the fourth. The most likely explanation is that participants are simply biased towards conservatism. Giving away large amounts of resources in real life is something that is discouraged, at least in most modern countries. If this is true, then what explains the generosity in the fourth experiment? The only obvious explanation for that seems to be individual variation between participants, and it should be borne in mind in this respect that, provided other participants are not actively ungenerous, only one participant needs to be actively generous to encourage such behaviour. And if they encourage it early enough in the game, then there are more resources to play with throughout.

An example of such encouragement can be seen in Game 3 of the Competitive condition in the second experiment. The generous giving seems to have started with one player, before spreading throughout all players. Can this explain what happened in the fourth experiment? Figures 8.1 and 8.2 show the pattern of gift giving throughout the two games. As generosity was apparent from early on, practice rounds are included. As can be seen, gifts are quite low in the first round of both games, and certainly not remarkably high. No player gave away everything, for example. However, the appearance and spread of generosity can be observed. For example, in the fourth round of Game 1 (see Figure 8.1) ${ }^{3}$ Player 3 stands out in his generosity. In this round, he was paired with his pseudo-teammate Player 4. In the following round, Player 4 is similarly generous to Player 2, who is rather generous in the following round. A similar pattern can be seen in Game 2, except

\footnotetext{
${ }^{3}$ Since there were three practice rounds, this makes it the first round of the game proper.
} 
it seems to start with Player 4 in the fifth round. ${ }^{4}$ It seems, therefore, that the unusually high mean gifts in these games may be explained by the presence in each of them of one player inclined towards generosity. The results from all the experiments together suggest that such individuals are relatively unusual, but the role of individual differences in generosity in this paradigm would be an interesting subject for future investigation. ${ }^{5}$

\subsubsection{Recognising team-mates}

\subsubsection{Feedback questionnaires}

Of the eight players in the Competitive condition, only three claimed in feedback questionnaires to have generally been able to identify their teammates, and two of these had managed to develop a Secret Handshake (see Section 8.4.1); the other mentioned other linguistic cues. One said he had had occasional success towards the end of the game. Half of the players reported no success. This is comparable to the second experiment, in which only half of players reported success at this. There were similar responses to the question, "Were you able to tell when you were talking to opponents?" Four said they had. Two of these had been the players using a Secret Handshake, and one of the others was their opponent, who mentioned this Handshake as a means of identifying them. Her teammate reported partial success only. The other player who claimed to have generally succeeded reported using linguistic cues to do so. Of the remaining four players, three reported no success, and one failed to answer the question.

\footnotetext{
${ }^{4}$ The seesawing that can be observed, whereby a player gives a lot away in one round, and far less in the next round, is partly explicable as a response to their last partner: while they gave away a lot in the previous round, they did not necessarily receive as much. Not only does this mean they have considerably less to give away in the next round, but that they may be temporarily discouraged from doing so.

${ }^{5}$ There is evidence that a variety of factors can influence cooperative behaviour, such as gender and anonymity (Dufwenberg and Muren 2006), observation (Haley and Fessler 2005), training in economics (Frank et al. 1993), and levels of the hormone oxytocin (Zak et al. 2007). Nevertheless, participants in this experiment were no more or less anonymous or under observation than participants in previous ones. Oxytocin levels, understandably, were not ascertained. Of the two players who seem to have started off the generosity in these games, the first was male and the second female. The first had, in fact, studied economics, although Frank et al. (1993) found an effect in the other direction. However, economic training might have helped this player realise sooner than average that generous gift-giving was a good strategy.
} 


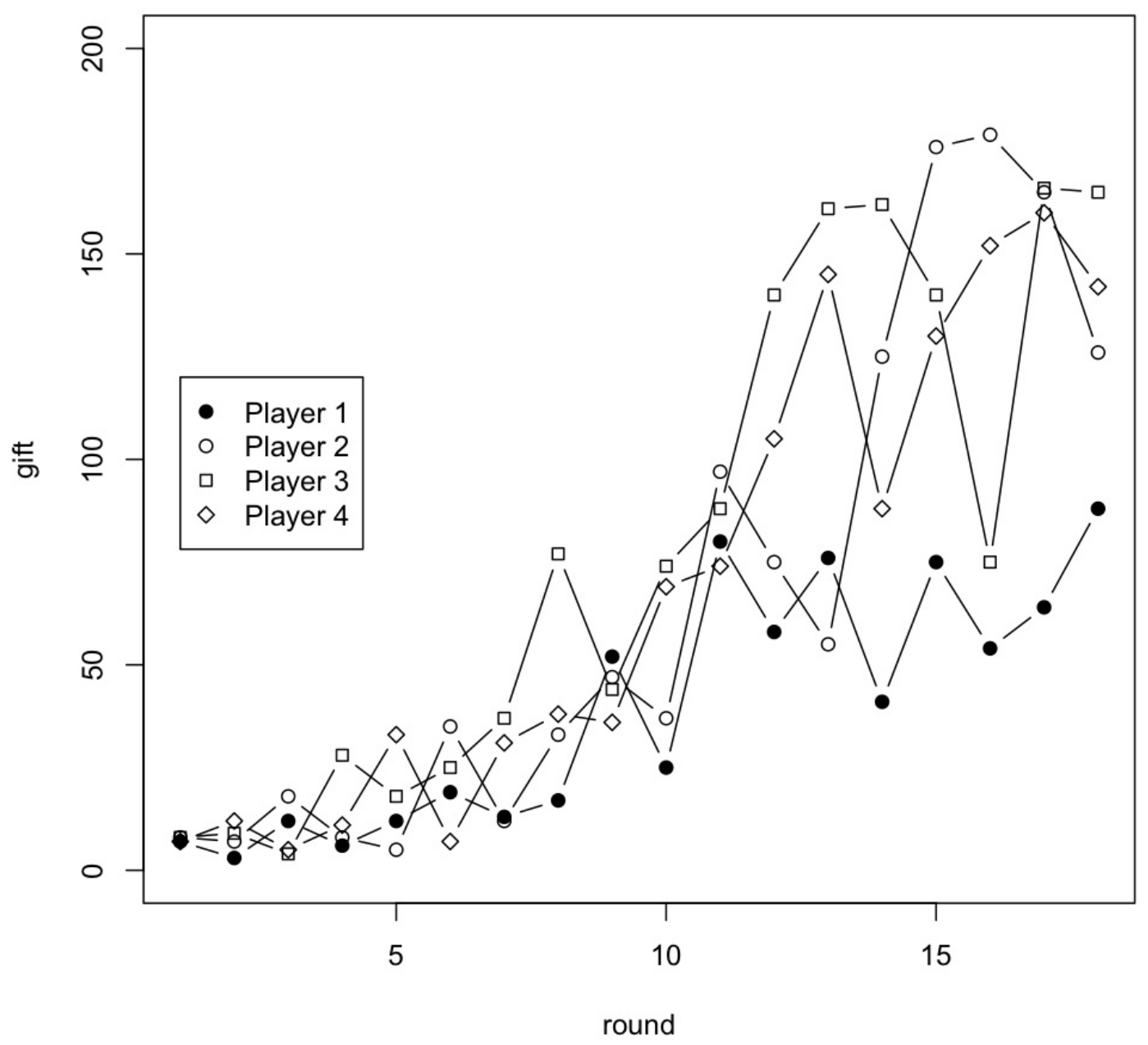

Figure 8.1: Gifts per round in Game 1 of the Cooperative condition 
Chapter 8. Explaining variation in gift size: a fourth experiment

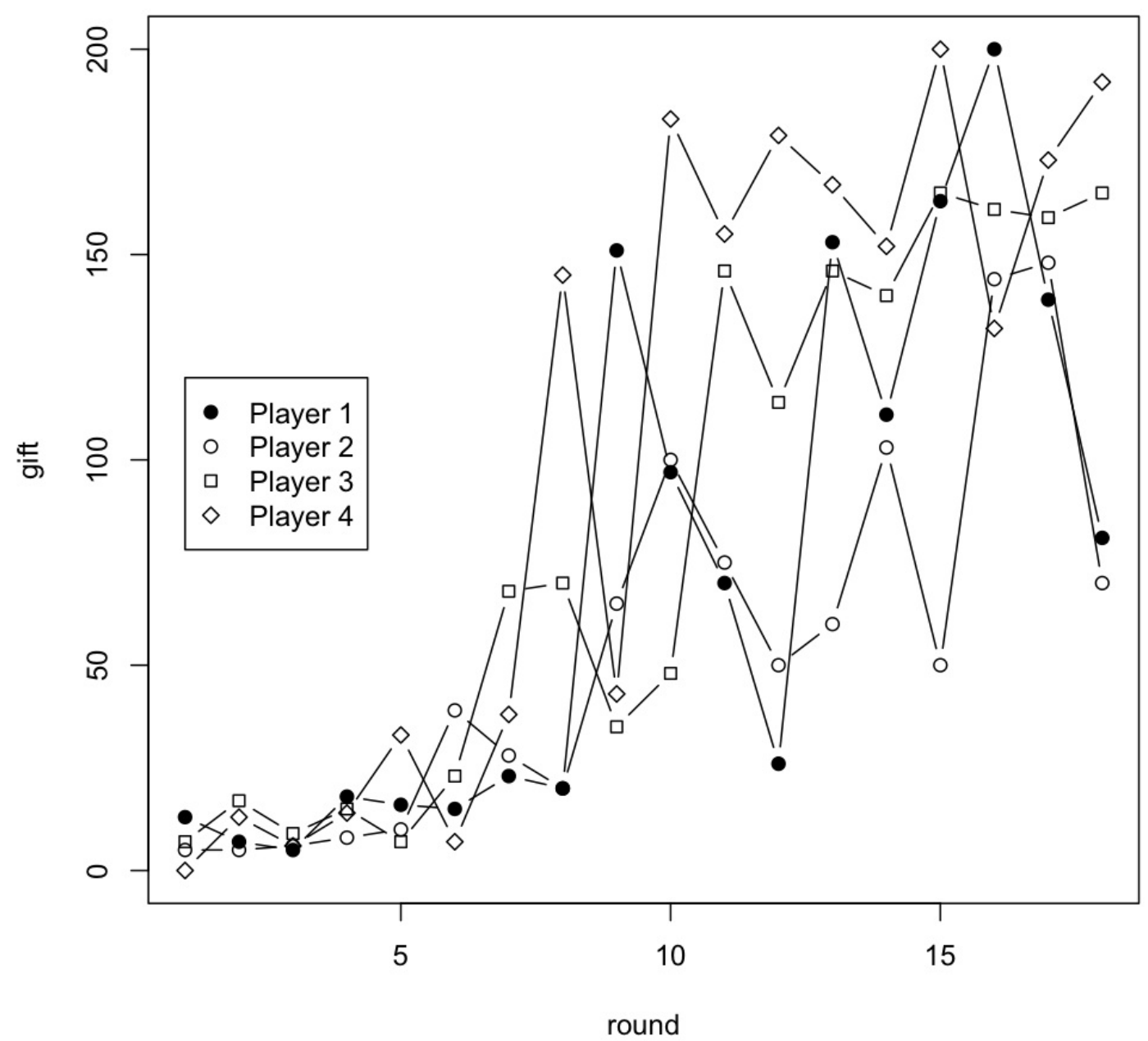

Figure 8.2: Gifts per round in Game 2 of the Cooperative condition 
In the Competitive condition, there were only a couple of intriguing responses to the question, "Could you tell (or guess) anything about who your team-mate was (e.g. gender, age, identity, nationality etc.)? If so, how?":

- (By a female participant) "No. But he is quick and polite."

- (By a male participant) "Not very good at the game. Very impulsive."

The responses in the Cooperative condition were more interesting:

- (By a male participant) "I think I guessed the gender because I reckon girls are more polite."

- (By a female participant) "I thought the player with all the exclamation marks, repetition was female." (cf. Section 7.2.1.1)

- (By a male participant) "One player was friendly to the point of flirty ... I hope it was a girl ... I think you could tell gender by politeness, punctuation, jokey tone."

- (By a male participant) "Perhaps some of the males in the group may have been more aggressive."

\subsubsection{Explicit and implicit judgements in the Competitive condition}

There were 6 failures in this experiment to answer the question, "Were you talking to a member of your own team?" representing $0.05 \%$ of potential responses. Only one of the four teams had a perfect record.

As can be seen from the third column of Table 8.2, players were more often right than wrong, which is significant only if players are assumed to have a $50 \%$ chance of being right $(S=31$ incorrect out of 106 responses; $p<.001$ ); the same caveats apply as in the second experiment (see Section 6.3.1.2), and again players could have done similarly well by guessing no every time. One team, however, did succeed in employing a Secret Handshake (see Section 8.4.1), and it can be assumed on that basis that members of this team actually succeeded in recognising each other. Members of this team made 22 definite guesses, of which only two were incorrect. In both games, the team that made the most definite guesses won. 
A Wilcoxon $T$ test revealed no significant difference between gifts to teammates and gifts to opponents in those rounds where players guessed they were paired with opponents $\left(N_{1}=7\right.$ and $\left.N_{2}=54 ; p>.05\right)$.

There was no significant difference between the time spent chatting in the Competitive condition (mean: 140.43) and in the Cooperative condition (mean: 143.12; $t$ $=-0.85, d f=109.1, p=0.198)$. It cannot be assumed that this reflects a significant difference between the second experiment (where there was a significant difference) and the third and fourth experiments. As the CHAT stage was 20 seconds shorter in the third and fourth experiments, the difference may reflect a ceiling, whereby players are more inclined to stop chatting in the last 20 seconds of the stage.

\subsubsection{Divergence}

In this experiment, there were 40 potential variables in each condition (20 words in each game $\times 2$ games). In the Competitive condition, 25 of these had more than one variant to them by the end of the game, and 28 in the Cooperative condition. 144 new variants were introduced in the Competitive condition (giving a mean of 5.76 variants in every variable set), and 142 in the Cooperative condition (a mean of 5.07 in every set). This is similar to previous experiments.

The same method was used to measure convergence and divergence in the alien language as was used in previous experiments. In no game was there a significant between-team difference in within-team convergence, and in neither condition was there significant divergence between teams (Competitive: $p=.364$; Cooperative: $p=.306)$; nor were the two conditions significantly different $(p=.29)$. This is consistent with the results in the second experiment. Similarly, there was no significant syntactic divergence in either condition (Competitive: $p=.66$; Cooperative: $p=.65$ ), nor any significant difference between conditions in this respect either $(p \approx 1)$.

Results for synonym pairs were similar to those in previous experiments. For the want/need pair: 181 to 19 (91\%) in the Competitive condition and 229 to 35 $(87 \%)$ in the Cooperative condition. The imbalance in the grain/corn pair was less 
striking, however: 69 to $29(70 \%)$ in the Competitive condition and 91 to $47(66 \%)$ in the Cooperative condition.

\subsubsection{Comparison with score}

In both Competitive games, the winning team was the team that did best at recognising teammates and the most generous. The data points, however, are too few for inferential statistics to be meaningful.

\subsection{Strategy}

\subsubsection{Identification strategies}

All four strategies used in the first and third experiments were used again here:

- Dialect Recognition (DR);

- Intentional Innovation (II);

- $\operatorname{Mimicry}(\mathrm{M})$;

- Secret Handshake (SH).

Team 1a's Secret Handshake began in the first round of the game proper, and simply involved each player greeting each other, repeating the last character of the word for "hello" (nopa). For example (from the eighth round):

Player 0: nopaaaaaaaaaaaa

Player 1: nopaaaaaaaaaaa

They also tended to do the same for "goodbye" at the end of an interaction. This time, their opponents did imitate the greeting, and had a little success. However, they were not completely successful at this. When Team 1a were actually paired up together, they tended to respond to the question, "Were you talking to someone on your own team?" by voting 4, meaning, "Definitely", and they would give each other correspondingly large amounts of resources. When tricked by their opponents, on the other hand, they were more cautious, voting 3, and giving away some resources, but not as many as to each other. The Handshake was not quite foolproof, therefore, but it was effective. In the eighth round, having realised their opponents had caught on, Player 2 followed his greeting with a smilie - :) - and Player 1 responded with 
the same. In subsequent rounds, this became an obligatory part of their Handshake. Player 3 caught on to this in the eleventh round, and fooled Player 2 completely. In the twelfth round, however, Player 1 was not to be fooled so easily. It seems the clue lay in Player 3 not making nopa long enough. In her feedback questionnaire, Player 1 described the greeting, and said both players had to make it the same length. In fact, this did not occur in any interaction in the game! However, there was never a difference of more than two letters between members of Team 1a. This was something that Team $1 \mathrm{~b}$ never caught on to.

In spite of the cleverness of this Handshake, it is striking that Team 1a did not get the highest score in the condition; Team 2a scored more. This is particularly interesting given that this team apparently did not employ any such sophisticated Handshake. Moreover, Player 2 of Game 2 reported that he initially found it difficult to recognise his teammate (though he said this was mainly because they were paired up rather infrequently). His teammate, however, seems to have been especially adept at identifying relatively subtle cues. As well as learning to recognise one of her opponents by his use of ropa for repa to mean "I", she noticed that her teammate tended to type faster than everyone else.

\subsubsection{Summary of results}

As in the second experiment, only half the players claimed to have been generally successful at identifying their teammates; this time, however, there was no sign of a gap between explicit and implicit judgements of identity. Nor did the alien language show any significant tendency to diverge. Gift giving was at a similar level in the Competitive condition in this experiment as in the second experiment. In the Cooperative condition, however, gifts were strikingly large. This is most likely due to the presence of a particularly generous player in both games, whose generosity spread to the other players.

All the main strategies identified in previous experiments were used again in this one. This experiment contained the fourth example of the most interesting of these, a Secret Handshake. 


\subsection{Discussion}

With respect to the two main research questions of the experiments so far-can players identify their teammates, and does their behaviour in the game lead to socially structured divergence in the alien language? - this experiment supported the findings of the previous three experiments.

The main reason for carrying out this fourth experiment was to determine whether or not the small differences between the second and third experiments, in round length and in instructions, might have influenced gift-giving behaviour. The results from this fourth experiment are slightly ambiguous. As regards the Competitive condition, if the unusually high mean gifts from Game 3 of the second experiment are ignored, then the overall mean gift for the fourth experiment is slightly higher, but still lower than the overall mean gift in the third experiment. This suggests there may be a very small effect from the instructions, a conclusion that may be further supported by the difference in the number of players attempting to sabotage their opponents by giving them a large number of resources. However, it is worth bearing in mind that a team in the fourth experiment succeeded in devising a Secret Handshake. This made recognising teammates relatively straightforward, and the level of frustration present in the second experiment, which, it was suggested, might have encouraged "malign generosity" towards opponents, was not present here. In any case, there is no evidence at all to suggest that the differences between the second and third experiments had any influence on the answers to the main research questions. 


\section{Chapter 9}

\section{Discussion of experimental results}

\subsection{Summary of findings}

Four experiments were carried out, which can be categorised as High- or Lowfrequency conditions depending on whether players were paired with their teammates for half the rounds (High-frequency) or only a third of them (Low-frequency). The first and third experiments were High-frequency experiments, and the second and fourth were the Low-frequency experiments. The second, third, and fourth experiments, moreover, consisted of two conditions each: a Competitive condition, in which players in a game were divided into two teams of two, and a Cooperative condition, in which the four players were all on the same team. There were two main research questions:

Recognition: Were players able to distinguish between their teammates and their opponents?

Divergence: Did the strategies they used to play the game lead the alien language to diverge?

The answers to both questions depended on the relative frequency with which players interacted with their teammates; the answer to the second question depended in addition on whether there was a pressure for players to make the distinction mentioned in the first question. In the High-frequency conditions only, players did significantly better than chance at identifying their teammates. In the Low-frequency conditions, players' success can be explained in terms of their 


\begin{tabular}{lll}
\hline & Low-frequency & High-frequency \\
\hline Competitive & $\begin{array}{l}\text { Poor recognition; } \\
\end{array}$ & No divergence. \\
& ${ }^{\mathrm{c}}$ & Divergence. $^{\mathrm{d}}$ \\
\hline Cooperative $^{\mathrm{b}}$ & No divergence. $^{\mathrm{c}}$ & No divergence. $^{\mathrm{d}}$ \\
\hline
\end{tabular}

Table 9.1: Summary of results for conditions

a See Sections 6.3.1 and 8.3.2.

b See Sections 5.4.1 and 7.2.1.

${ }^{c}$ See Sections 6.3.2 and 8.3.3.

${ }^{d}$ See Sections 5.4.2 and 7.2.2.

simply having realised that they were more likely to be paired with their opponents than their teammates (although there was some evidence that players in the second experiment did better at implicit identification than they were aware; see Section 6.3.1.2). Only in the High-frequency Competitive conditions did the alien language show signs of significant divergence. Only in these conditions, that is, were players significantly more like their teammates than their opponents in their use of the alien language. Most interestingly, while every player in the High-frequency Cooperative conditions was paired twice as often with one other player than with either of the others, this frequency bias did not lead these pairs to diverge significantly from the other players. Table 9.1 summarises these results.

The results suggest, first, that human beings are very adept at using variation in language (and potentially other cultural systems) to create markers of identity and to infer social information about other individuals, at least given a minimum level of interaction with members of their own community; moreover, they can do this very quickly (the experiments lasted about an hour each). Second, they suggest that a pressure to use language in this way influences its cultural evolution, causing members of the same group to converge with each other and to diverge away from members of disfavoured groups. The results do not show that such a pressure is a necessary condition - that is, that divergence can never occur through variation in the frequency of interaction alone. Indeed, the results show that frequency of interaction plays an important role in causing divergence (which did not occur in the Low-frequency conditions). It would, moreover, be rather surprising if biases in frequency of interaction did not lead to divergence on their own over long time 
periods. What the experiments suggest, however, is that a pressure to use linguistic variants to signal identity speeds up divergence. In evolutionary terms, this all looks rather as if it could be understood in terms of drift and selection (cf. Section 2.4.3 and Chapter 4), and it would be similarly misguided to assume that biological speciation events can occur only as a result of the latter. Section 9.5 will return to the question of analogies with biology (see also Chapter 4).

\subsection{Bridging the alien-human gap}

Gilbert and Troitzsch (2005), with reference to simulations in the social sciences, make a distinction between "explanatory" and "predictive" models, the difference chiefly lying in the level of detail modelled, which influences the degree to which accurate predictions can be made. "Phenomenological" models, by contrast with both, attempt simply to model the phenomenon in question without any claim to explanation or prediction. The experimental model presented in this thesis is at least explanatory, since it has a claim to be modelling a real-life phenomenon. It is predictive in the sense that it allows a broad prediction to be drawn from it, specifically that a pressure for social marking will lead to faster linguistic divergence. At this level, however, any explanatory model can be argued to be predictive, and the model does not make any claim to be a simulation of a particular real-life situation. ${ }^{1}$

Yet, if the model is to have a real claim to being explanatory, the connections between it and the real world must be made clear. The scenario with which participants are presented at the start of the experiment (see Appendix A) makes this somewhat explicit. The population being modelled is one that was previously relatively well integrated and linguistically homogeneous, but which becomes divided in two for some reason. What precisely the teams might correspond to in real life is open to interpretation. The Biblical story in which the Gileadites tested the Ephraimites by asking them to say "shibboleth" (Judges 12:5-6) is a classic example whose relevance to this area of research has not gone unnoticed (see e.g. Kinzler

\footnotetext{
${ }^{1}$ How could it, given the abstractions necessary for the experiment to be carried out? It is doubtful whether narrowly predictive models of language change can be implemented at all, except computationally.
} 
et al. 2007: 12577). This is a case of social significance being attached to a linguistic variant, of which there were certainly examples in the experiment.

\subsubsection{Serbo-Croatian disintegration}

The shibboleth story is not a story of language divergence as such, however: the divergence had already occurred before the events took place. Where, then, to seek real-life cases of linguistic divergence in which social divergence plays a causal role? On the face of it, the twentieth century provided several political situations that somewhat resemble the social structure in the Competitive condition of the experiment - a group splintering into two or more parts. An example of the linguistic consequences of such events is provided by David Norris's (1993) Teach Yourself Serbo-Croat, which became, in 2003, Teach Yourself Croatian. On the face of it, this is evidence of language divergence in a single decade. In reality, of course, it is not language divergence so much as rebranding (see Greenberg 2004 for a detailed study of the four-way disintegration of Serbo-Croat), and it is nothing new. Much linguistic ink, after all, has been spilt for decades on the question of what it means to call something a language (for a discussion of the issues, see Chambers and Trudgill 1998: 3-12; Croft 2000: 13-20). The extent to which it has actually influenced the distribution of linguistic variants, which remains primarily geographically conditioned, is doubtful (Norris, personal communication), and work remains to be done. Nevertheless, there have certainly been efforts among some Croatian linguists, for instance, to emphasise the differences between Croatian and Serbian and to consolidate a distinct standard (focusing primarily, for the sake of relative ease, on written rather than spoken language) - both enterprises that have been the source of considerable controversy (Greenberg 2004: 115-34). Such linguistic engineering should not surprise anyone who has read a popular history of the English language; modern American spelling owes much to Noah Webster, for example.

This is not, however, all that similar to what happened in the experiments. The divergence seen there is not best explained as the result of such intentional behaviour (this will be discussed in more detail in Section 9.3). Indeed, while some of the differences between British and American English are explicable with reference to lexicographers and linguists, this cannot account for anything near the majority 
of differences between the two dialects. The Balkan linguistic situation is an attractive real-life situation to relate to the experiment, and is currently a popular topic for research. It may well be, furthermore, that political events of the last two two decades have sown the seeds of greater future divergence between the languages involved. Nevertheless, it is also a good example of why simulations, both computational and experimental, must play an important complementary role in the study of language change and variation. While we can make tentative predictions about future divergence between Balkan languages, it will be a long time before we see it for sure, and picking apart the factors that bring it about will be difficult. There is, after all, already variation between groups of speakers: Serbs and Croats can already tell each other apart linguistically. This removes a certain pressure for socially based divergence. The experiment provided something that is impossible to find in real life: a language that was initially homogeneous, where every speaker initially received identical input. This is an advantage of the model, and of models generally, because it allows the researcher to abstract away from complicating factors. It does, however, make the model that much less similar to real-life cases - in real life, the input to multiple speakers is never identical. This is not to imply that simulations should not be made to relate to the real world. The point is merely that to do so is far from trivial, and that points of difference between models and reality are not necessarily drawbacks.

\subsubsection{Classic sociolinguistic studies}

The caveat from the previous section being borne in mind, other real-life linguistic cases are worth mentioning. Chapter 3 made reference to Labov's (1963) study of change in Martha's Vineyard. The results of the experiment are very much in line with Labov's findings here, and they should not be taken necessarily as contradicting his later downplaying of the role of identity in language change (see Labov 2001: 191, 506; Section 3.3 of this thesis). The experiment did not produce divergence as a result of frequency of interaction alone, but this does not mean that it would not have occurred given more time. The point is that social pressures significantly increase the rate of divergence. 
The other case worth mentioning here, also discussed in Chapter 3, with particular reference to work by Trudgill $(2004,2008 a, b)$, is that of New Zealand English, or rather of colonial dialects in general. The development of this kind of dialect presents a particularly interesting area for research. It involves both convergence between settlers and divergence from the people they left behind, as well, in certain cases, as between different groups of settlers. There is, furthermore, the advantage that these dialects began developing over a century ago, meaning that there has been enough time for significant change, but not so much time that the origins are lost in the mists of history. Indeed, the New Zealand case is made particularly fascinating for research purposes by the existence of recordings, made in the 1940s, of the first generation of native New Zealanders (see Gordon et al. 2004 for a detailed account of this and the development of this dialect in general). The starting point in such cases is also much cleaner than in many cases of dialect formation-Trudgill (2004: 26) refers to "tabula rasa situations". One must be clear: the linguistic starting position can hardly be called homogeneous in these cases - but, as noted before, it practically never can - the advantage of the colonial setting is that the dialects settlers bring with them are likely to lose a considerable amount of their original geographical and social significance (Trudgill 2004: 2), and they do not encounter any local dialects of the same language. For Trudgill, of course, this dialect contact is the key to explaining new colonial dialect formation. It is worth stressing again (see Section 3.3.1) that this view has much to recommend it. The question is whether identity can so easily be rejected as a plausible factor in dialect formation. This was discussed in Chapter 3, and the discussion will not be repeated here. The experiment can, however, be understood as a model of colonial dialect formation, where at least some contact remains between settlers and those who remain behind. For such cases, it predicts that, where there is a pressure for settlers to distinguish themselves and to be able to recognise outsiders who speak the same language (and, it must be stressed, this need not involve the establishment of a coherent new national identity), then we should expect this pressure to hasten the formation of a new colonial dialect. This, moreover, occurs through accommodation and alignment between speakers, not in spite of it. 


\subsubsection{Midlan High}

All the examples of real-life dialect formation discussed so far have been relatively large-scale. Such a focus may lead us to overlook somewhat smaller-scale everyday instances of socially grounded linguistic divergence. This is in fact ubiquitous, and familiar to most people who recall the social pressures they experienced as adolescents. Indeed, there is evidence that this age group is one of the most influential in terms of the propagation of linguistic variants (Kerswill 1996; Kerswill and Williams 2000).

Emma Moore spent three years in the company of female members of one year of a Bolton high school, which she refers to by the pseudonym "Midlan High" (Moore 2003, 2004). Here she observed a social split into two groups, defined by attitudes and behaviour: the "Populars" and the "Townies", the latter being more inclined to engage in such behaviours as drinking and drug taking (although the Populars also engaged in the first activity), and the former more inclined to play an active role in the life of their school. As might be anticipated, Moore found that the divergent social practice was reflected in "increasingly divergent linguistic practice" (Moore 2004: 385), with regard to four variables that she examined: nonstandard were, tag questions, negative concord and right dislocation. She makes an important point about this divergence, however. On the face of it, her data identify "nonstandard were and negative concord as Townie variants, tag questions as a Popular variant and right dislocation as a neutral sociolinguistic marker" (ibid.: 390). However, this obscures the fact that none of the girls involved rejected any of the variants completely. To ignore the Popular girls' use of nonstandard variants would be to fail to recognise that these variants, and the extent to which they used them, played a role in their own identity. The same variants, in other words, meant something different for the two groups of girls, and that social meaning was expressed not only in their use of the variants, but in the frequency with which they used them.

This nuanced view should be borne in mind in interpreting the results of the experiment. It was not the case that players could be divided into categories as "users of variant x" and "users of variant y". There were, granted, a few cases of variants being used only by members of one team (the Secret Handshakes were a very salient 
example of this), but this was not the norm. Context, moreover, was important. As the first utterance of an interaction, "jori noloku" in Game 5 of the first experiment had a very particular significance (see Section 5.5.1); used later on simply to mean "I have", it did not have this significance. Its use as a greeting, moreover, depended on who its user thought they were talking to. In general, it should not be forgotten that every player had to talk to every other player, and the alien language could not be split at the end of any game into "Team 1 words" and "Team 2 words". The divergence, measured in terms of relative distance between players, was based on the proportions according to which players used particular variants, not on categorical differences. What is more, there was considerable intra-team variation, and it should not be assumed that player's identities in the Competitive conditions of the game were entirely team-based. In both Competitive and Cooperative conditions, it is clear from feedback questionnaires that players noticed individual variation between all three of the other players, and were conscious of the ways they themselves differed in their use of the language not only from their opponents, but also from their teammates. In addition to this, players' use of particular variants was conditioned by the immediate context. Section 9.3 will discuss the role of mimicry, along with other strategies in the game. Even in the Cooperative condition, however, players were influenced in their use of language by their partner in the round; the role of accommodation, its contribution to divergence, and the ways in which it was affected by competitiveness and frequency of interaction will be examined in Section 9.4.

\subsection{The role of players' strategies}

Sections 5.5, 6.4, 7.3, and 8.4 discuss the strategies that players used in the Competitive conditions. As discussed in these sections, four main linguistic strategies can be identified:

- Dialect Recognition (DR): try to remember how different players use the language and identify them based on this.

- Intentional Innovation (II): make changes to the language that are likely to be recognisable. 
- Mimicry (M): try to sound as much like the person you're talking to as possible.

- Secret Handshake (SH): say something at the beginning of the interaction that identifies you to your team-mate (and ideally not your opponent), and requires a specific response from them

The relationship between these strategies and the divergence that occurred in the High-frequency conditions is worth exploring. None of these strategies is explicitly about causing the alien language to diverge; the closest we get to that is in Secret Handshakes, which involve two members of the same team coordinating with each other to produce a multi-turn variant not shared by members of the opposing team. However, to the extent that this is about causing the language to diverge, this stops at the greeting. Furthermore, the divergence calculated was based on wordlevel variation (see Appendix C), so variants that stretched over more than one conversation turn, as these Handshakes did by definition, did not contribute to it. Indeed, since Secret Handshakes were generally so successful in creating a safe environment for teammates to exchange resources, we might even expect there to be less overall convergence between teammates who used them, though this occurred in only one case (see Section 7.2.2), which should therefore be treated with caution.

This suggests that the overall divergence, if it has anything to do with players' strategies, is better explained by the other three strategies. Again, however, it must be stressed that there is no reason to assume that any of these strategies were employed with the purpose of creating divergence; their purpose was to recognise one's own teammate and to appear to be the teammate of one's interlocutor (the extent to which a distinction was made between teammate and opponent in this respect is discussed in Section 9.4). The relation between this and the linguistic divergence that arose in the game is best understood in terms of Keller's (1994) invisible-hand model of language change, in which the small-scale behaviour of individuals contributes to the emergence of large-scale phenomena, without any intention on the part of the individuals that these phenomena be created. For this reason, straw-man characterisations of the role of social factors in language change that rely on teleological mechanisms (some are mentioned, for example, in Section 3.3) should be treated with care. This, in general, is an argument for 
the utility of cultural-evolutionary explanations of language change as a means of shifting focus from the speaker to the language. In the history of linguistic thought, for example, a focus on speakers led to language universals being taken as evidence for a highly complex, and - as Kinsella (2009) argues - evolutionarily implausible, set of language-specific constraints in the human brain. A focus on language as an adaptive system in its own right, on the other hand, makes clearer the possibility that language universals can be understood as the result of adaptation to the human brain, rather than the other way round (Lass 1997; Kirby et al. 2004; Evans and Levinson 2009; Chater et al. 2009).

Such a shift in focus, however, does not in any way imply that the divergence seen in the game was not the result of players' strategies. How, then, do the linguistic strategies listed above contribute to divergence? Their role is best understood if the distinction between innovation and propagation (see Section 3.1.2) is borne in mind. Intentional Innovation clearly plays a role in the first: it is one means by which new variants get created. The other means is accidental innovation. The majority of new variants arising in the games can most likely be accounted for by the second, rather than the first, process. There are a few cases where innovations look clearly deliberate, and many players claimed to have deliberately innovated, but there is no reason to reject the more parsimonious assumption that this accounts for a relatively small proportion of new variants, and that the majority of them arose as a result of errors in production and recall.

If innovation, whether intentional or accidental, accounts for the appearance of new variants, mimicry accounts for their propagation. Whether it accounts for it entirely depends on whether the term is understood to refer to all cases in which a variant is copied from one individual to another (in which case, this is trivial, since that is the only means by which propagation can occur) or if it refers only to intentional imitation, as might be expected from its inclusion in the list of strategies. The distinction is important. As discussed in Section 3.3.1.2, speakers in a dialogue can be expected to automatically align themselves linguistically (and not only linguistically; see Pickering and Garrod 2004; Garrod and Pickering 2007) purely for the sake of establishing common ground, regardless of whether or not they want to signal affiliation. If players do want to signal affiliation, then we 
should expect greater alignment, whether consciously or unconsciously. Lakin and Chartrand (2003), for example, showed experimentally that a pressure for affiliation increases rates of "nonconscious mimicry" of behaviour, and that individuals who have previously failed to affiliate themselves to a group subsequently exhibited more mimicry than individuals who had previously been more successful. Lakin et al. (2008) showed similar results, and demonstrated, moreover, that excluded individuals mimicked in-group more than out-group members (see also Williams et al. 2000, 2005, and, for a review of research into imitation more broadly, Hurley and Chater 2005). In addition to this level of unconscious mimicry, several players explicitly stated that they had deliberately attempted to imitate both their teammates and their opponents (see Section 9.4 for more discussion of this). In this way, variants were propagated between players.

We can, as discussed in Sections 3.2 and 3.3, expect that the propagation of variants will be biased by variation in the frequency of interaction and by social factors. Both were manipulated in this experiment. The division of players into teams in the Competitive conditions led players to employ the strategy referred to above as Dialect Recognition. This is how variants acquire social significance: there is in language, it must be quite clear, no intrinsic social meaning attached to any particular variant (just as there is no intrinsic semantic meaning attached to any particular phonetic variant). ${ }^{2}$ Instead, variants must become associated mentally with particular individuals or groups, which means that some association outside the minds of hearers - that is, a correlation - must be recognised between variants and speakers. Again, such associations may be recognised on a conscious or an unconscious level (and this is the source of the distinction drawn in sociolinguistics between stereotypes and indicators). In any case, the recognition that certain

\footnotetext{
${ }^{2}$ It must be added, in point of contrast, that some variants may be intrinsically liable to attract certain meanings (otherwise there would be no onomatopoeia) or certain aesthetic valuations, which will affect their distribution. We might expect uvular and pharyngeal sounds, for example, to be less common in the world's languages, owing to the association of the relevant articulators with snoring, gargling, choking and coughing up phlegm-English speakers, for example, often make reference to phlegm in disparaging references to languages with "guttural" consonants. Indeed, it turns out that these sounds are rather uncommon in the world's languages. Only 19.1\% of languages in the WALS Database, for example, have uvular consonants (Maddieson 2008). This is intended, it should be noted, merely as a suggestive anecdote. Little serious work has yet been done on the association between typological frequency and aesthetic judgement. Such valuations, moreover, are not social in the sense meant here.
} 
variants are more likely to be associated with one team than the other encourages variation in their use.

\subsection{The role of accommodation/interactive alignment}

Had the alien language diverged in the High-frequency Cooperative condition as well as in the Competitive condition, then the divergence would be best explained with reference to interactive alignment and frequency of interaction alone (cf. Trudgill 2004, 2008a). The fact that it did not is evidence that the pressure to distinguish between teammates and opponents played an important role, and influenced the degree to which players accommodated to each other. As discussed in Section 9.3, there is evidence that human beings unconsciously vary the degree to which they mimic others' behaviour depending on their sense of affiliation, and that they do this selectively depending on the person they are talking to (Williams et al. 2000, 2005; Lakin and Chartrand 2003; Lakin et al. 2008). There are in fact two ways in which the pressure for social marking might have influenced rates of accommodation:

1. Players in the Competitive condition accommodated more to their teammates than to their opponents.

2. Players in the Competitive condition accommodated more to everyone than in the Cooperative condition.

The second possibility would account for the divergence owing to the biased frequency of interaction. The fact that there was no divergence in the Low-frequency conditions is not evidence against the first explanation, however, since players did so badly at recognising when they were talking to their teammates. The fact that it is advantageous in the game (and, broadly speaking in real life) to always appear to be on the same side as one's interlocutor seems to be a good motivation for preferring the second explanation, and certainly, as shown in Tables 5.1, 6.2, 7.2, and 8.2, many players claimed to have deliberately tried to imitate their opponents.

However, we might still expect there to be a bias in accommodation. Attempts to align oneself with members of another group come in different sorts. It has long been the case in Britain, for example, that a child born into the working class canas a result of education and career path - join the middle class quite legitimately. 
In this context, the consequences of not quite having the right accent can vary, and may well affect employment opportunities, but it tends (on the whole) not to lead to violence. If an individual fails in an attempt to trick their way into another group, on the other hand, then the consequences range to the considerably more serious, as the history of espionage reveals (and as shown in the Biblical shibboleth story). In this latter case - which is closer to the situation in the game - it is better not to even attempt to accommodate to one's opponents unless one is sure of one's imitative abilities. It is better to be taken for an honest outsider than a deceitful one; better, in other words, to be captured as a prisoner of war than as a spy. It is even worse to be taken for a spy by your own people; if one is rejected by one's opponents, there is at least a chance of getting home. If one is rejected by one's own, then there is no home left. ${ }^{3}$ There is real-life pressure, then, to accommodate primarily to one's own group and to be cautious about accommodating to one's opponents (which, in itself, can lead to rejection by the home group), and we might expect this to lead to a general bias which would influence players' behaviour in the game. However, it should be borne in mind that the pressure in the game itself cannot, clearly, be quite as strong as in real life. For reasons of ethics, no in-game punishment could ever be anywhere near as great as real-life rejection, or the punishments meted out on captured spies. For this reason, we cannot expect players to be as cautious. There is a further point to be made, which concerns the alien language. There are several advantages to using a small artificial language in the experiment: it limits explicit strategising; it makes the initial linguistic state of all players clear and relatively equal; it allows language change to be observed over a very short time period. Without it, in other words, the experimental results would have been considerably less interesting. However, the fact that the language is not native to any of the players and, moreover, was learnt by all of them during the half hour before the experiment began makes attempting to imitate one's opponents less risky. In real language it is relatively easy to recognise a poor attempt to mimic

\footnotetext{
${ }^{3}$ The case of the social climber is a little different. Frequently the adoption of the cultural practices of a group to which one has a legitimate claim to belong can lead to rejection by the group one grew up in. This is evidently hard, but at least the rejected individual is likely to have somewhere to go. The worst scenario is to be accepted by neither group.
} 
one's own accent; in the game, accents had not had long enough to develop to be so clearly fixed in the minds of players. ${ }^{4}$

And yet, in spite of this, players seem to have had a strong sense that there was a right and a wrong in the language, stating in feedback that some other players "had a better grasp" of it, or "made a lot of mistakes". Stereotypes, or at least markers in the sociolinguistic sense (see Section 2.4.1, footnote 6), also developed, with players recognising distinctive variants used by other players, or with the other team as a whole. There are, then, multiple pressures both to accommodate to every other player and to accommodate more to one's teammates. Nor should it be assumed that all players behaved the same; as seen in Section 7.2.2, at least one team employing a Secret Handshake converged less than other players. Since, moreover, there was individual variation in the strategies that players employed and the degree to which they employed them, it seems likely that there should have been individual variation too in the relative degree to which players imitated teammates and to opponents, whether consciously or unconsciously.

This is as far as such speculation can get us; it is time to look at what light the data can shed. The following analysis was undertaken: every time a non-canonical word form (i.e. a form not included in the original wordlist) was used by both partners in the CHAT stage of a round, the second user of the form was taken to be accommodating to the first. Consider, for example, the following exchange (from the Competitive condition of the third experiment), in which $k$ is an abbreviated variant of kazuru, meaning "I" and $v$ is a variant of venu, meaning "want":

Player 1: datu

Player 3: datu

Player 1: k hanete konura

Player 1: e kofu

Player 1: $\mathrm{k}$ hanete kofu

Player 3: hanate rogu

Player 1: v rogu

\footnotetext{
${ }^{4}$ As shown, for example, in Section 7.3.1, some variants arose in the game that were also very hard to imitate accurately. These super-reliable markers represent a minority of variants, however. More generally, it would be fascinating to carry out an iterated version of this game, where variation between teams was maintained across generations.
} 
Player 3: k v kanura

Player 3: pafa v?

As can be seen, Player 1 uses these abbreviated forms from early on in the round, and by the end of the round, Player 3 is also using them. With respect to these variants, we could say that Player 3 is accommodating to Player 1. For any variant in a round, the first such case of accommodation was categorised depending on whether it was to a player's teammate or opponent (subsequent uses of the same variant in the same round were excluded to avoid the results being biased by rounds in which the same variant was repeated multiple times). Tables 9.2 and 9.3 give a summary. ${ }^{5}$ The last row of each table gives the mean number of words accommodated to per interaction.

As the tables show, the difference between accommodation rate to teammates and accommodation rate to opponents seems mostly to be explained by frequency of interaction; in the Low-frequency conditions (the second and fourth experiments), players interacted a third of the time with their teammates and two thirds of the time with their opponents, and this is reflected in accommodation rates, where players accommodate roughly a third of the time to their teammates. Similarly, in the High-frequency conditions (the first and third experiments), players interacted half the time with their teammates and half the time with their opponents, and the accommodation rate is not far off $50 \%$ for each. There is, however, a striking difference in the third (High-frequency) experiment between the Competitive and Cooperative conditions (in fact, this is the greatest difference between any two of the average scores per round) showing that players accommodated more overall in the Competitive condition $(p<.001$, based on a one-tailed binomial test). This supports the second hypothesis given above, that the divergence in this condition is explained by players' accommodating more overall, rather than by players' accommodating more to their teammates specifically. The same is not seen in the second experiment, where the difference between overall accommodation rate in the Competitive and Cooperative conditions is rather smaller $(p>.05)$. This suggests, interestingly, that while a pressure to distinguish between teammates and

\footnotetext{
${ }^{5}$ These scores exclude data from the practice rounds, in which players had wordlists in front of them.
} 
opponents increases the rate of accommodation, it requires that players be reasonably confident about making such distinctions. It is intriguing, however, that there is a very slightly significant difference between conditions of the fourth experiment, but in the other direction $(p=.046)$. If this is meaningful, it is most likely related to the particularly high rates of gift giving in this condition (see Section 8.3.1), and it would be interesting in future research to investigate the connection between generosity and accommodation more closely.

\begin{tabular}{lllll}
\hline & First & Second & Third & Fourth \\
\hline To teammate & 144 & 73 & 163 & 23 \\
\multirow{2}{*}{ To opponent } & $(58.5 \%)$ & $(31.3 \%)$ & $(55.3 \%)$ & $(34.3 \%)$ \\
& 102 & 160 & 132 & 44 \\
Total & $(41.5 \%)$ & $(68.7 \%)$ & $(45.6 \%)$ & $(65.7 \%)$ \\
\hline Mean per interaction & 1.76 & 1.55 & 1.84 & 1.12 \\
\hline
\end{tabular}

Table 9.2: Summary of accommodation rate (Competitive conditions)

\begin{tabular}{llll}
\hline & Second & Third & Fourth \\
\hline To teammate & 72 & 87 & 29 \\
To opponent & $(34.1 \%)$ & $(50.3 \%)$ & $(32.6 \%)$ \\
& 139 & 86 & 60 \\
Total & $(65.9 \%)$ & $(49.7 \%)$ & $(67.4 \%)$ \\
\hline Mean per interaction & 1.41 & 1.08 & 1.48 \\
\hline
\end{tabular}

Table 9.3: Summary of accommodation rate (Cooperative conditions)

It seems that the first hypothesis also has some explanatory role to play. A one-tailed binomial test shows a significant difference between the rate of accommodation to teammates and to opponents in the Competitive conditions of the first and third experiments (first: $S=144, p<.01$; third: $S=163, p<.05$ ). This suggests that players did accommodate significantly more to teammates in these conditions than to opponents. There is no such significant difference in any other condition of the experiment. 
In summary, it appears that the divergence seen in the High-frequency Competitive conditions is chiefly to be explained by greater overall accommodation in the Competitive conditions, but that players in these conditions also accommodate somewhat more to teammates than to opponents. Intriguingly, however, a certain level of confidence in one's ability to distinguish between teammates and opponents seems necessary before players increase their accommodation. This is surprising, since it is beneficial to always seem to be on the same team as the interlocutor of the moment. It may be that players simply do not see it in those terms, and treat accommodating to their teammates as a different strategy from imitating their opponents; and where they cannot make the necessary distinctions, they implement neither.

\subsection{Social selection}

Section 2.4.3 introduced the term "social selection", defined as the (conscious or unconscious) selection of variants on the basis of their social significance. The role of social factors in language change was discussed in rather more detail in Section 3.3, and Chapter 4 discussed the degree to which selection need imply a memetic model of cultural evolution, and concluded that this was not likely to be a useful model except in a relatively limited context. This does not mean, however, that terms like "selection" are any less useful, merely that it may be misleading to assume that the processes involved are the same on the level of variants as in biological evolution.

So where did selection occur in the experiment? Most obviously, it occurred in production. This is what is involved in Mimicry and in the accommodation described in Section 9.4: players (whether consciously or unconsciously) used one variant in preference to another to accommodate to their interlocutors, in some cases (where this is identified as a deliberate strategy on the part of the players) explicitly because this allowed them to be identified as members of a particular team. This can be seen particularly in the Secret Handshakes: a particular greeting or response is selected because of its significance in identifying the speaker's affiliation.

We can be clear from the data and the participants' feedback that this kind of selection occurred. It is important to note, moreover, that it is contextual. As was 
made clear in Section 9.4, players accommodated to both teammates and opponents; this means that social selection occurred with regard to the immediate discourse context. Even when players thought they were talking to their opponents, and recognised variants as being associated with those opponents, they selected these variants. The selection bias here can be summed up in Keller's (1994: 100) maxim to "talk like the others talk", on which Trudgill is so keen (e.g. Trudgill 2004: 27). However, to the extent that players accommodated more to their teammates than to their opponents (see Section 9.4), there was another overarching bias, to "talk especially like your people talk". This still involves differential selection of variants at the point of production; but it also involves a different kind of selection. When a speaker selects a variant on the basis of its association with their interlocutor, it does not seem unreasonable to employ evolutionary terms and say that the speaker's language is adapting to its immediate environment. Given this, and given that speakers apparently vary the degree to which they do this between environments, then there is also selection of accommodation-degree, and this in turn will be influenced - in real life - by selection of environments. ${ }^{6}$

A further point is worth making, which recalls the discussion in Section 3.1.2.3. It was stated above that a speaker's language adapts to its immediate environment; this, as noted, is selection at the point of production. Assuming that the variants a speaker hears and produces influence their competence, and that there is some frequency effect involved, then we should expect the various biases described above, over time, to influence speakers' competence - and the probability with which they are likely to produce a given variant (since this is obviously not entirely predictable on the basis of accommodation alone; speakers do not simply sound like their interlocutors). Since production depends on competence, of course, this will feed back into interactions and exercise its influence on the variants that are propagated.

Debates about multilevel selection in biology boil down mainly to the question of whether such phenomena as group selection and niche construction are necessary levels of explanation, or if the data they are invoked to account for can be equally

\footnotetext{
${ }^{6}$ This was mentioned in Section 3.3.1.2 as the relatively uninteresting case of social factors influencing accommodation indirectly. In the game, of course, players in the game were denied the opportunity to choose who they were paired with. It would be interesting to give them this opportunity in future research.
} 
well accounted for in a gene-centric view (Traulsen and Nowak 2006; West et al. 2007; Lehmann et al. 2007; Wilson 2007). Similarly, this section has discussed various different evolutionary pressures on language change. While it may well be the case that biological evolution is best understood from a gene's-eye-view, it does not seem true to say (as was discussed in Chapter 4) that the different pressures operating on language change can be usefully reduced to a memetic account, even if such an account can be a useful lens through which to view certain linguistic phenomena.

\subsection{Conclusion}

The experiments described in this thesis provide good support for the role of a pressure for social marking in speeding up language divergence. This pressure works by influencing the degree to which speakers align with each other in communicative interactions, which varies according to both social context and to the identity of the interlocutor. This chapter also attempted to bridge the gap between the experimental findings are real-world speech communities. It did not, however, attempt to put the findings in the broader context of the evolution of language. This will be done in Chapter 10. 



\section{Chapter 10}

\section{An evolutionary perspective}

The experimental work described in Chapters 5 to 9 of this thesis is primarily about modelling divergence in modern language. However, there are clear evolutionary dimensions to it. First, as discussed in Section 9.3, a cultural-evolutionary perspective on the results of the experiments is illuminating, since it shifts a focus from an interpretation that puts a potentially misleading emphasis on players' strategies (see also Chapter 4). Second, there is the biological perspective. Chapter 2 put sociolinguistic marking in the context of the evolution of cooperation, giving an ultimate explanation for why accents and dialects matter. That chapter did not, however, go so far as to sketch an account of what this adds to our understanding of how language evolved, in the sense of the biological evolution of the apparatus that allows us to use language. Section 10.4 will go some way towards doing this, albeit tentatively - the amount that can be said with certainty about our distant evolutionary past is limited, particularly with regard to such a phenomenon as language, which is unique in nature, and leaves no fossils (cf. Christiansen and Kirby 2003). This does not mean, of course, that researchers have avoided such questions, and Sections 10.1 to 10.3 will critically examine several claims about human evolution relevant to the central question of this thesis.

\subsection{A bias towards language}

It seems likely that the drive to attach socially relevant interpretations to biological and cultural cues is, at least in its most basic form, the result of biological evolution. Human beings are far from being the only animals to do this (Beecher 
1982; Halpin 1991; Komdeur and Hatchwell 1999), and we must suppose that we acquired the basic ability relatively early in our evolutionary history - most likely as a development of kin-recognition mechanisms (see Section 2.2.1.1). It also seems inescapable, however, that for modern human beings the behaviour has a significant learnt component. The ways in which human beings can differ from each other is enormous, and it would be ludicrous to expect to find all the details of human variability written in the genome. Indeed, the most parsimonious assumption is that human beings are equipped with a keen eye for variation and a bias towards cooperating most with close kin and individuals with attributes in common with kin. ${ }^{1}$ Knowledge of the relative reliability of specific kinds of cues can be acquired through learning.

Experiments described in Kinzler et al. (2007) provide evidence for an innate social bias towards traits associated with close kin. In these experiments, 5- to 6month-old infants from American-English-speaking families looked reliably longer at images of adult women who had previously been seen speaking American English, compared with women who had been seen speaking Spanish or American English played backwards (the same effect was not seen where the women's faces were replaced with animated geometric shapes). Infants were also more likely to choose toys apparently presented to them by speakers of their own language. This is good evidence that preference for one's mother's language appears very young, though that in itself is not new (see e.g. Moon et al. 1993). More interestingly, it shows that this influences social behaviour. However, there is no reason on the basis of this particular study to assume that language is a special case, and that the results do not simply reflect a broader bias towards all traits associated with one's mother, or with close kin more generally.

A later study (Kinzler et al. 2009) presents evidence for an intriguing subtlety in children's social preferences. In these experiments, 5-year-old monolingual American-English-speaking children from predominantly white neighbourhoods were

\footnotetext{
${ }^{1}$ There may, of course, be some (well defined) cases where interacting with outsiders is preferable, such as in mate choice, where high levels of inbreeding can be harmful (see for example Bateson 1983b, and in particular Bateson 1983a, for a discussion of this). This does not contradict the main point.
} 
shown pairs of pictures of other children and, in most conditions, played a recording, apparently of the children speaking. For each pair, the participants were asked which of the two they would prefer to be friends with. There were two independent variables: the race of the children (black, white, ambiguous) in the pictures and the language of the recording (French, American-accented English, French-accented English, silence). Accent turned out to be more important than race for making friend choices: specifically, children preferred to be friends with silent children of their own race, but preferred native-sounding children of another race over foreign-sounding children of the same race. Interestingly, children did not distinguish significantly in their preferences between children speaking French and children speaking Frenchaccented English, suggesting that "children do not grant 'native' social status to people who communicate in their native language, yet speak with a foreign accent" (627).

These results are convincing. A further claim made by the researchers - that this reflects an innate rather than a learned bias - is less convincing, however. It is, after all, quite possible that the participants had had more exposure to children of other races, presented as members of their own group, than to children with different accents (on television and in films if not in real life). The researchers were aware of this issue, and attempted to answer it with a further experiment that replaced race with visual distortion (where the height:width ratio was changed from 1:1 to approximately 2:3), and found that children still preferred accent. This is a clever response, but it remains problematic. First, the aim was presumably to present the participants with images of children who look different from themselves in an unfamiliar way. The example pictures given in the paper, however, look more like distorted photographs of ordinary children than ordinary photographs of oddlooking children. It is, moreover, likely that most of the children were somewhat familiar with the idea of faces that had been visually distorted in this way, from fairground mirrors, television, and so on. In any case, it cannot be assumed that the children took the pictures to represent the children as they looked in real life; no evidence is given that they did. It can be countered that, presented only with the pictures and no linguistic cues, participants preferred to be friends with the children in undistorted images. This is indeed notable; however, all it means is that, given 
no other information, the participants chose the children whose picture looked most normal. They had, after all, to choose one of them. The second problem is that, even if the participants accepted the distorted pictures as pictures of odd-looking children, then their reaction can still be accounted for in terms of learnt behaviour. Even if the participants had never seen faces distorted in this particular way before, they might still have learnt that children who look different in some way should still be accepted as part of the group (indeed, this is a message many parents are very keen to instil in their children). In this context, after all, "race" means little more than "salient physical difference".

The researchers are not claiming that human children are innately biased to treat head shape in particular as of low social importance. Instead, they are claiming two things. In both Kinzler et al. (2007) and Kinzler et al. (2009) they claim that we prefer to interact with individuals similar to our close kin, and that this bias can be shown at a very young age, suggesting that it is innate. The existence of kin recognition and imprinting mechanisms in other species (see e.g. Sluckin 1973; Hepper 1991) suggests that we should expect as much, and these studies provide compelling evidence for it. Their second claim, made only in Kinzler et al. (2009), is more controversial: that we are innately biased to pay particular attention to linguistic cues. This remains entirely possible, and, if the bias exists, it could potentially be relatively ancient - it may, for example, relate to conspecific vocalisations, rather than to language per se. However, while the experiment described above shows elegantly that such a specific bias exists in some form, at least in modern American children, it does not succeed in showing satisfactorily that this is innate, rather than learnt, behaviour. It is a very promising basis for a broader research project, however, and if the results could be replicated cross-culturally, and accent compared with a broader range of variables, both natural and artificial, then this would be very interesting indeed. Such a study is, in fact, currently being planned by members of the Max Planck Institute for Evolutionary Anthropology.

Constructing a plausible evolutionary story remains a difficult task, and Kinzler et al. are on very shaky ground when they attempt to relate their results to a more specific account of human evolution: 
Given that social groups in ancient times likely differed in accent, but not in race, children may be predisposed to rely primarily on accent to guide their social evaluations of novel individuals. (Kinzler et al. 2009: $632)$

This is indeed consistent with their findings. Unfortunately, not only would the opposite scenario also be quite consistent with them, but the scenario described would fit equally well, if not better, with different findings. We know from experience that human beings do not simply ignore race, and, indeed, there is experimental evidence that amygdala responses to human faces are influenced by the interaction between the race of the face and the race of the participant (Hart et al. 2000). As noted above, we mean little more by "race", in this context, than "salient physical difference"; this is why Kinzler et al.'s (2009) use of distorted photographs made any sense at all. All these experiments confirm that people take note of salient physical difference, and they show that children take it into account in making friends. In the scenario described above, any person who looked obviously different would very clearly be an outsider, and could be recognised as such before they even opened their mouths. We might thus expect race to remain a very important marker. If we expect any distinction between race and accent given the scenario above, we might perhaps expect that, if human beings were able to make subtler distinctions in one of the two areas, it would be in accent. If people of different races are less frequently encountered than people with different accents, then a strong association of "otherness" with anyone who looks different would be enough; it would be more important to distinguish between five subtly different accents. But even this is not absolutely clear. What "differed in accent, but not in race" means is that the level of linguistic variation was greater than the level of physical variation among the individuals one would most often expect to encounter. In this case, if there were any observable physical differences at all between social groups (and we might expect there to be some, at least given some degree of reproductive isolation and inbreeding), they would be particularly subtle, and more subtle than differences in accent (since language changes faster than physical attributes). We might thus expect human beings to have a subtler sense of physical than of linguistic difference! This is if we assume any innate difference at all. It might equally be the case - and this would be more useful - that human beings are flexible enough to 
learn subtle distinctions in whatever system necessary. It is certainly quite clear that subtler distinctions between accents must be learnt, and that speakers are not immediately adept, on hearing two unfamiliar accents, in distinguishing well between them. American-English speakers, for example, tend to find it difficult on arrival in the UK to distinguish between different British regional accents. It is thus not clear that the scenario described above would lead us necessarily to expect the results that Kinzler et al. (2009) found - it is not even clear what it would lead us to expect with any confidence. Their findings are in fact more obviously consistent with a scenario in which people have come to live in multiracial societies, where racial variation is familiar, and no longer as reliable an indicator of being an outsider as accent. This is the situation in the modern United States, where the experiment was carried out. It is of course true, as suggested in Section 2.4.2, that accent is in general a more reliable indicator of group membership than biological traits, but this does not mean that human beings are innately disposed to recognise this fact.

Kinzler et al. (2009) present an elegant study with very interesting results. It remains, moreover, entirely possible that human beings attach especial social importance to conspecific vocalisations, or even to specifically linguistic behaviour. This would be very interesting and, indeed, entirely consistent with the main claim of this thesis. In the absence of good evidence, however, our default assumption should be that we are biased only to prefer individuals who are like us in some way, and that the relative importance we attach to different biological and cultural cues is learnt rather than biologically evolved behaviour.

\subsection{Selection for variability}

The central question of this thesis concerns the influence of a biologically evolved behaviour on cultural evolution. It is interesting to ask, however, whether there has been influence in the other direction. In other words, has the usefulness of language as a source of cultural markers led to a selective pressure on the biological evolution of the language faculty; has there, in other words, been selection for variability? Dunbar (2003: 230) argues that there has: 
After all, if language evolved to allow individuals to exchange information, one might expect stability to be an important consideration ... since it is not beyond the wit of evolution to have produced language structures that are more resistant (if not totally resistant) to corruption in this way, the implication is that the corruptibility of language is precisely the whole point (and has been deliberately selected for).

Dunbar's premise, that we should expect considerable pressure for an unchanging language, echoes Labov (2001: 5): "It is hard to avoid the conclusion that language, as an instrument of communication, would work best if it did not change at all." Obvious as this statement may sound, it is misleading. Even if we characterise communication very narrowly as the transmission of propositions, changes of environment as a result of migration or technological innovation uncontroversially introduce a need for new lexical items. In fact, Labov notes that additions of vocabulary, along with prestige forms, are a special case; his explicit focus is on "alterations in the mechanism of the language" (ibid., 6). There are other reasons to treat the lexicon as different: first, the addition of lexical items is not associated with the same negative reactions as structural change (ibid.); second, it is accepted in all linguistic theories that the lexicon can continue to be added to throughout a speaker's lifetime, while grammatical structure is assumed to consolidate at a relatively early age, and to be rather resistant to change thereafter - though the degree to which this is the case and the basis for the phenomenon is still very much subject to debate (Newport et al. 2001; Abrahamsson and Hyltenstam 2008).

So while changes in the lexicon can be directly attributed to the need to express new ranges of propositions - that is, to environmental change - it is harder to find examples of structural linguistic change (Labov's "alterations in the mechanism") that have the same basis. Models like that described in Kirby (1999) and Kirby et al. (2008), however, assume just such a change: the introduction of structure to an unstructured proto-language to allow speakers to express novel meanings. It could of course be countered that this study is concerned with the very early stages in human language - a movement from something we might not call language to something that we would - and that Labov is concerned with modern language. This is true, but extensions of paradigms by analogy (e.g. the regularisation of the past tense of English climb) are a smaller-scale example of a very similar process, 
and these are both common in modern language and frequently deprecated in their early stages.

Structural change can also result from changes in technology. The invention of writing, like other forms of recording, allowed utterances to be preserved out of context; it might be expected that this would lead to structural changes to compensate for the lack of contextual information. Wray and Grace (2007) make this argument, and suggest further that we should expect changes in population structure to be reflected in language change, as small close-knit speech communities have more common ground to rely on and, therefore, less need for explicitness. Recording technologies like writing can be seen as a special case of this, since they again open up the language to a wider audience. Wray and Grace's claim is supported by empirical work by Lupyan and Dale (2010). It is a useful approach, since it may help in answering a classic hard problem in historical linguistics: the "why then?" question (cf. Weinreich et al. 1968). For example, the development of the different ways of talking about the future in English are fairly well understood in terms of grammaticalisation and semantic change. Considering, however, that speakers of earlier forms of English managed to go about their daily business happily without such forms, and that many millions of speakers of different modern languages do likewise, it is not clear what, at different points in the history of English, changed to make the need for such forms more compelling. It seems likely, to the extent that there are identifiable factors that contributed to these tipping points, that changes in technology and population structure are among them.

It is, then, far from obvious that even a language designed solely for communicating propositions should not be expected to change. Moreover, it is not the case that language communicates only propositions. It seems reasonable to say, for example, that a speaker with an Edinburgh accent is communicating their connection with Edinburgh. This hangs somewhat on one's definition of communication. Scott-Phillips (2008) is a useful discussion of how the term can be defined (with respect not only to language), and concludes, in line with Maynard Smith and Harper (2003), that it should be defined in adaptive terms, where both the signal and the response must be adaptive. It may be argued on the basis of this definition 
that there is a difference between the two cases such that, while the transmission of propositional information is adaptive on both sides - and thus constitutes a signal in the strict sense employed by Scott-Phillips (2008; see also Diggle et al. 2007) - the transmission of social information is best treated as a cue, where only the response is adaptive. This is due to a (potential) difference in the intentions of the speaker, who usually intends to transmit the propositional content, but may not intend to transmit the social information. There are two responses to this: first, the speaker may very well intend to convey the social information, so this is at least a case of a signal some of the time; second, adaptation must be understood not only on the level of the signaller's intentions, but also on the level of the evolution of the signal itself. In both cases, the association of a particular meaning with a particular combination of sounds, be that meaning social or non-social, is the result of cultural-evolutionary processes. As it is hoped this thesis has shown, the fact that a particular combination of sounds is associated with the social meaning "Edinburgh" (or whatever) is the result of adaptation. It seems highly likely that language is designed to transmit social information as much as it is designed to transmit propositions.

The communication of social information relies on great variability in language. Social groups change rapidly, at least by comparison with the rate of technological change, and other environmental changes, through most of human history. To some extent this is Dunbar's point; Dunbar, however, contrasts two communicative functions of language - communication of propositions, and communication of social information - and suggests that one leads us to expect stability, while the other does not. For reasons given above, however, it should not be assumed that this is the case; it may be that human communication in general is best served by a relatively high minimal level of variability.

Since this question concerns selective pressure for variability, it is on the earliest stages of human language that we should again focus our attention. The claim that there has been pressure for variability in human language can be understood in two ways, depending on one's model of how we came to have language (see also Section 4.1): 
Biological faculty: the structure of human language is primarily the result of biological evolution and language-specific selection. (e.g. Pinker and Bloom 1990; Hauser et al. 2002)

Cultural tool: Human language is primarily a cultural artefact. (e.g. Kirby 1999; Everett in preparation)

The first model implies a relatively high level of genetic specification of the language faculty. Within this model, "selection for variability" can be understood either in terms of a barrier to greater specification (which seems to be what Dunbar is implying), or as a pressure for a very highly specified system to becomes less highly specified (cf. Deacon 2003; Yamauchi 2004). The second model implies that, while humans must be biologically ready (or pre-adapted) for language, there is relatively little in the genome that is language-specific; language, rather, arises out of (and adapts to) more general cognitive and physical characteristics. To some extent, this model can be understood in terms of the "barrier to greater specification" mentioned above. The advantage of this second account, however, is that it makes the pressure for variability fundamental to the nature of language; in Kirby's (1999) model, for example, it is because of its ability to adapt culturally that language is language. Its ability to adapt to later cultural innovations is more convincingly, and more parsimoniously, explained with reference to this than on its own terms.

The question that lies behind these two models concerns the degree to which the innate knowledge that constrains language learning is language-specific, and it has been the subject of decades of fierce debate (see Sampson 2005 for a detailed discussion of the history and the main arguments involved). Answering it conclusively will require a great deal of further research in psycholinguistics, neuroscience, and genetics.

\subsection{Bilingualism and maturational constraints}

There has long been good evidence that children find it easier to acquire native-like competence in a language than adults (Penfield and Roberts 1959; Lenneberg 1967). Children are even able to acquire native-like competence in more than one language at the same time, and without fundamental delay or confusion (Petitto and Holowka 
2002; De Houwer 2009). The standard explanation accounts for the difference in terms of a "critical", or "sensitive", ${ }^{2}$ period during which individuals are better able to acquire language. The fact that children are very good at acquiring native-like competence and adults are not contributes significantly to the reliability of speech patterns as a marker of identity. There is clearly no reason to suppose, however, that the reduced ability among adults was selected for on this basis. Indeed, there are very obvious advantages to being able to acquire native-like competence in new languages and dialects in adulthood; the reduced capacity to do so must be explained either in terms of some disadvantage to retaining the capacity (which may relate to brain plasticity in general, rather than to language acquisition specifically) or in terms of some inevitable reduction in plasticity, perhaps related to the physical or chemical properties of the brain. The use of speech patterns as a marker must be parasitic on this reduction; it cannot be the reason for it.

There are arguments, however, that the difference between children's and adults' ability to acquire language is related to human social behaviour. Hagen (2008a) claims, on the basis of archeological evidence, that:

All human cultures have two moral codes; one that applies intra-culturally (to "us"), and one that applies inter-culturally (to "them", whoever they may be). Throughout history, humans have been capable of committing atrocities against others that would be considered morally offensive if committed against one of their own. (55)

This leads him to the conclusion that, while it is is highly advantageous to rapidly learn to communicate with other members of one's own group from a young age, "there would have been no substantial benefits" from being able to acquire new languages later in life (57). This is clearly compatible with the evolutionary claims made in this thesis, but not exclusively so. It is important to be able to distinguish outsiders from insiders even if our default response to the former is not violent. Hirschfeld (2008), indeed, is highly critical of Hagen's claims and proposes to the contrary that "the ease with which children simultaneously learn multiple languages evinces a state of sustained cooperation and exchange between groups" (183; this is

\footnotetext{
${ }^{2}$ This term is preferred by some (e.g. Oyama 1978; Patkowski 1980) who wish to avoid making a strong claim about the criticality of maturational constraints. It should also be noted that there are in fact assumed to be different sensitive periods for different aspects of language (Neville et al. 1992); vocabulary acquisition continues in all speakers into adulthood.
} 
discussed in more detail in Hirschfeld 2001). Certainly he is right to point out that what is meant by late bilingualism is often not what is meant by childhood bilingualism: the former need only involve learning one language in adulthood, having already acquired one's first language; childhood bilingualism, on the other hand, tends to involve simultaneous learning (ibid.). A lot depends on the importance of this distinction. It certainly seems, on the face of things, that acquiring two language simultaneously is an impressive task. If it is indeed impressive enough that we must assume it to be an adaptation, then this points towards an evolutionary history in which children were often required to be bilingual. As Hisrchfeld suggests (ibid.), this seems, at least on the face of it, more consistent with peaceful movement of women between communities than with the capture of women as "spoils of war". Hirschfeld may, however, be relying too much on the apparent impressiveness of early bilingualism. Certainly within a generativist framework, which assumes that children approach the acquisition task with a great deal of prior linguistic knowledge, and that the differences between human languages are relatively superficial (cf. Hagen 2008b: 187), acquiring two languages is not necessarily much harder than acquiring one. In usage-based models, the main alternative to generative models, we should also not expect children to be specifically adapted to learn more than one language at once, since such models generally assume only minimal specific adaptation to learn one! Until we can say with any real clarity how much harder we should expect the task to be, we cannot assume that bilingualism provides evidence for any special adaptation, or any basis for making inferences about our evolutionary history.

Hagen demands less from language acquisition research: for his claim it is enough that children do considerably better than adults at acquiring native-like competence; and this is held to be the case by most researchers in the field. Nor does his claim rely on any special adaptation of the language faculty. The point is rather that, while it may seem extremely useful for adults to be able to acquire nativelike competence in new languages, the ability is obviously not useful enough for evolution to have given it to us. After all, adults are perfectly able to learn new languages, at least well enough to engage in social interaction and trade. The cost to a child of failing to learn any language is far far greater than the cost to an 
adult of failing to learn a second one. This, indeed, appears to be all we need to explain the relative poorness of adult language learning, if we assume on more general principles that brain plasticity decreases with age (see e.g. Nieto-Sampedro and Nieto-Diáz 2005). There remain problems with Hagen's argument, however. First, as noted above, other historical scenarios are entirely compatible with the data. All the data suggest is that the pressure to acquire native-like competence in a new language has historically been significantly greater for children than for adults. Yet, while Hagen is right that moral attitudes to within-group relations are different from moral attitudes to between-group relations, this does not necessarily mean that inter-group relations have been predominantly violent. A great deal of entirely amicable interaction between groups is perfectly possible, provided at least some members of both groups are able to get some sort of communication off the ground, and there are ample examples in history of amicable, or at least non-violent, interactions between different groups based on even quite rudimentary levels of communication.

Indeed, all this seems to be somewhat missing the point: learning an entirely new language is a difficult task even for children; as Hagen himself says (2008b: 187): "In reality, it takes about two years of sustained (not occasional!) exposure for a child to become a fluent speaker of a language." In that time and with that kind of exposure even an adult can get good enough at a language to get by. And getting by is good enough; sounding like a native may get you accepted faster, but if you are able to spend at least two years in sustained contact with members of another culture, then the problem of being accepted is likely already to have been solved.

There is a related question: learning an entirely new language is uncontroversially time-consuming; but what about learning a new accent or dialect of a language one already speaks natively? This, moreover, is likely to be of more frequent use: two groups who speak only slightly differing dialects of the same language are likely to be in relatively close proximity to each other. It would surely be useful for members of one group to occasionally pass themselves off as members of the other, and any individual who could do so would surely have a selective advantage. Why then, if this is likely to be advantageous, are adults not better at imitating nonnative accents? The most obvious reason is that learning to imitate another accent 
convincingly, without sustained exposure to it (by which time the learner has been identified as an outsider), is a very difficult task that should not be underestimated. There is simply a great deal to learn. The actors and impressionists who achieve it today (and many do not achieve it well enough to satisfy a native) do so only with considerable training and practice. This is why identification based on accent is so reliable: an accent is a very complex marker. And it is important to get it right: to get it almost right, but not quite, is to risk being identified as an impostor. This is the worst result. It is better to be seen as an honest outsider than as a dishonest intruder (see also Sections 2.4.2 and 9.4).

\subsection{A tentative sketch}

It is time to sketch some sort of evolutionary scenario for the origins of sociolinguistic marking.

It seems to be the case, first of all, that human beings have a general ability to observe physical and behavioural traits in conspecifics and compare them on the basis of these with members of their own group. It seems furthermore to be the case that we are biased towards interacting with individuals who are most like members of our own group. There is good reason to suppose that both the ability and the bias are innate, and that they considerably predate the emergence of Homo sapiens; it seems likely that they evolved originally for kin recognition, and mechanisms of kin recognition are common throughout the animal kingdom (a detailed discussion can be found in Hepper 1991). There is even some evidence for it, albeit controversial, in plants (Dudley and File 2007; Klemens 2008; Dudley and File 2008). There is no strong reason to suppose, therefore, that this behaviour is especially connected with language. First, it seems likely that vocalisations were used for this purpose before the advent of language; certainly vocal kin recognition occurs in other species - it is the most common means of kin recognition in birds (Sharp et al. 2005), and it occurs in many, though not all, species of primate (Halpin 1991: 245; Rendall et al. 1996). Second, vertebrates use kin recognition cues from all sensory modalities (Halpin 1991: 231), and several different kinds of cue are used among primates (ibid., 245-7); humans seem to use the same broad range of cues for this purpose as they use to distinguish between individuals (ibid.). 
It remains possible, of course, that human beings are innately predisposed to pay linguistic markers special attention, and Kinzler et al. (2007, 2009) provide evidence for that. As discussed in Section 10.1, however, their results fall short of showing conclusively that this is an innate bias. As was argued, to show that would require a more extensive, and very carefully designed, cross-cultural study. If such a study were to find that we treat linguistic cues as special, there would be an important further step to be taken in unravelling the evolutionary story. This would be to draw a distinction between language and speech; in other words, do we treat sign language the same way? If not, and it turns out that we treat speech as special, then the next question must be whether this is in fact true of speech, or of vocalisations more generally. While the sign-language question is relatively easy to test, this one is not. While nonnative accents can be straightforwardly compared with native ones, as in Kinzler et al.'s study, a four-way distinction of the sort shown in Table 10.1 is tricky, since it is not entirely clear what should go in the bottom rowso much of human vocalisation has been co-opted by language that it is not clear what a truly non-linguistic human vocalisation would be that was culture-specific.

\begin{tabular}{lcc}
\hline & In-group & Out-group \\
\hline Linguistic & Native accent & Foreign accent \\
Non-linguistic & Native babbling? & Foreign babbling? \\
\hline
\end{tabular}

Table 10.1: Four kinds of human vocalisation

The best answer, it would seem, is to look beyond our species. As noted above, there is evidence of vocalisation-based kin recognition in other primates (Halpin 1991: 245; Rendall et al. 1996); chimpanzees are also able to identify individuals on the basis of their vocalisations (Izumi and Kojima 2004). If it can be shown that both humans and chimpanzees treat vocal cues as especially reliable, then this is good evidence for such a bias being pre-linguistic. Chimpanzees may even show signs of social marking that goes beyond pure kin recognition: there is evidence for group-specific variation in wild chimpanzees that cannot be explained by habitat or genetics (Crockford et al. 2004). 
While Occam's razor urges us to treat those aspects of human language that we find in our closest relatives as prelinguistic in origin, the reverse is not true. We cannot assume that if something is present in humans, but absent in other primates, that it evolved after we had language, or even that it evolved for language. It is at least possible that the fine motor control human beings possess over their speech organs did not evolve specifically for linguistic communication, but rather to extend the range of possible non-linguistic vocalisations. Certainly, as researchers of sign language are keen to point out, the vocal-auditory channel (pace Hockett 1960) is not a necessary component of language. Proponents of a gestural origin for language ${ }^{3}$ have given various reasons why language is more likely to have started with the hands than the mouth, including, most prominently, greater continuity with other primates and a greater degree of iconicity, by which symbols might be bootstrapped. This seems very plausible, but explaining the transition from sign to speech in such a model is problematic (Levelt 2004; Emmorey 2005). The most obvious argument, that this left the hands free for other things, is rather weak. It is hard to believe that this can have constituted a significant enough evolutionary pressure for the development of the fine motor control over the vocal tract enjoyed by modern humans. This problem is avoided somewhat if the evolution of the vocal tract is explained non-linguistically, allowing it to take over the burden of language when it was already somewhat developed. ${ }^{4}$ Accounts based on sexual selection are one possible explanation (cf. Miller 2001), and it has been argued convincingly, through comparison with other species, that the descended larynx did not evolve for speech, but as a means of exaggerating body size (Fitch and Reby 2001). There is an advantage of parsimony in arguments by Dunbar $(1993,1996,1998)$, which explain a great deal with reference to an increase in social group size. In this account, human vocalisations took over some of the role of grooming, since this allowed more individuals to be groomed more quickly and more cheaply. A growth in group size also makes the identification of members of one's own group a harder task, which

\footnotetext{
${ }^{3}$ Kendon (2002) provides a good review of the historical development of this hypothesis, which is an old one (dating at least to the seventeenth and eighteenth centuries with Bulwer 1644, and Condillac 1746). Modern proponents include: Hewes (1976); Corballis (2002); Volterra et al. (2004); Arbib (2005); Tomasello (2008).

${ }^{4}$ Though we should almost certainly envisage this not as a sudden transition, so much as a parallel development where language evolved as a combination of gesture and speech (e.g. Brown 2010; Kendon 2010), and where the latter came eventually to dominate.
} 
makes reliable social markers more important (see Section 2.4.2). Potentially, a consequent need for greater subtlety in this domain - along with other pressures, as noted above - could have contributed to the development of finer control over the vocal tract. Testing this hypothesis is a hard task, however, although comparative studies (like Fitch and Reby 2001) are a potential way in, and computational simulations could shed light on its plausibility. It is, it should be noted, very consistent with the hypothesis discussed above that human beings are innately biased to pay especial attention to linguistic (or at least vocal) markers.

The use that modern humans make of language as a source of social markers almost certainly has its origins relatively deep in our evolutionary history, in vertebrate kin-recognition mechanisms. This exploitation of language by modern humans, as shown in this thesis, influences its cultural evolution. It is plausible that it also contributed something to the biological evolution of the vocal tract, although this is uncertain, as is the question of whether human beings are biologically predisposed to treat linguistic markers as special. Answering these questions will require a broad methodology, including cross-cultural experiments with human subjects, comparative studies of other species, and computational simulation. 



\section{Chapter 11}

\section{Conclusions}

The main question of this thesis, as stated in Chapter 1, is this:

Does the need for social marking lead languages to diverge into new dialects (or at least hasten their divergence)?

The results of the experiments described in Chapters 5 to 9 suggest that the answer is yes. Section 11.1 will summarise these results, Section 11.2 will summarise how the divergence came about, and Section 11.3 will discuss possibilities for future developments of this experimental paradigm (see Chapter 10 for a discussion of possible future research employing different methods).

\subsection{Summary of experimental findings}

\subsubsection{First experiment}

The first experiment, described in Chapter 5, was a useful proof of concept, which showed that language divergence could be modelled experimentally in the laboratory. The experiment drew inspiration from work by Nettle and Dunbar (1997) and Nettle (1999), who investigated a very similar question computationally, and from Garrod and Doherty (1994), who showed how the structure of paired interactions in a community of participants can affect linguistic coordination.

Participants played a game in which they typed messages to each other, using an artificial "alien language", to negotiate to exchange resources. There were two teams of two players in each game and there was an advantage to giving away 
resources to teammates. However, as the interactions were anonymous, players had to infer from the way their interlocutors used the alien language whether or not they were on the same team or not. The experiment showed that human beings are capable of coming up with useful sociolinguistic markers in under an hour in a language they have only just learnt, and suggested that this can lead the language to diverge significantly into socially structured dialects.

The experiment also revealed the linguistic strategies participants used to play the game. Three of these are relatively straightforward: Dialect Recognition involves paying attention to how other speakers use language; Mimicry involves trying to sound like other speakers; and Intentional Innovation involves deliberately creating novel forms by which you stand out to others, or at least to your teammate. The most interesting, and also the rarest, example of this was dubbed "Secret Handshake". This took the form of a greeting, or at least began with an utterance early on in the dialogue. This timing matters, since it is clearly preferable to establish identity as early as possible. However, what made it interesting was that the initial utterance required a specific response. Since opponents could only see one half of the handshake at any one time, imitating it was difficult. Such Handshakes, therefore, set up relatively secure environments in which players could exchange large amounts of resources with their teammates without giving too much away to their opponents.

However, although this first experiment produced impressive and intriguing results, it suffered from limitations. Players spent a relatively long time paired with their teammates at the start of the game. Moreover, although players only interacted with their teammates for half the game proper, this meant that every player was paired for twice as long with the one player who was their teammate than with either of their opponents. This did not allow a very certain conclusion to be drawn that the divergence was due to social selection. It might be better explained with respect to frequency of interaction.

\subsubsection{Second experiment}

In an attempt to resolve the questions left by the first experiment, the second experiment (described in Chapter 6) reduced the number of rounds at the start 
of the game in which players were paired with their teammates and introduced a control condition, in which all four players were on the same team. This experiment also changed the frequency of interaction such that every player interacted with every other player the same amount (in both conditions). This time there was no divergence (in either condition), and players did poorly at recognising their teammates. No team succeeded in establishing a Secret Handshake. This suggested that the divergence seen in the first experiment may have been the result of the relatively high frequency of interaction between teammates. However, the results did not show for certain that frequency of interaction was the only factor to play a role.

\subsubsection{Third experiment}

In order to see if a pressure for social marking might still also play a role, the third experiment (described in Chapter 7) took the frequency of interaction from the first experiment, but retained the control condition from the second. All of the strategies used in the first experiment were used in this one, and two teams employed Secret Handshakes; both of these scored very highly. As in the first experiment, there was a significant tendency for divergence in the Competitive (two-team) condition, and players did well at recognising their teammates. However, there was no significant divergence in the Cooperative (one-team) condition, even though every player in this condition was paired twice as long with one of the other players than with either of the other two. This is good evidence that, while frequency of interaction does play an important role in divergence, it is not sufficient on its own, at least given the time available. A pressure for social marking, in other words, significantly speeds up new-dialect formation.

\subsubsection{Fourth experiment}

There was a difference between the instructions for the second and the third experiments: although both sets of instructions mentioned that there was a penalty for unbalanced resources, those for the second experiment emphasised it more strongly. In case this had influenced gift giving, a smaller-scale experiment was run that used the instructions from the third experiment, but the frequency of interaction 
from the second. The results supported the findings with regard to the main research questions, and players in the Competitive condition behaved very much like those in the second experiment (although one team succeeded in employing a Secret Handshake). However, the Cooperative condition stood out in comparison not only with the Cooperative condition in the second experiment, but also with that in the third: players were extremely generous. This appears to have been the result of the presence of one particularly generous player in each game, who influenced other players early enough for generosity to take off as a strategy.

\subsection{How the divergence came about}

Since all players learnt exactly the same alien language, but no two players ended up using it in exactly the same way, all players can be said to have diverged from each other. This came about through innovation, some of which was a deliberate strategy. Players did not diverge equally and, where there was pressure for players to identify their teammates and sufficient interaction between them, teammates converged more with each other than with their opponents. This unbalanced convergence is how the between-team divergence came about (cf. Section 3.2).

The within-team convergence occurred through players' accommodating to each other. The Interactive-alignment model of dialogue (Pickering and Garrod 2004, 2006; Garrod and Pickering 2007) suggests that this should occur to an extent in any interaction as a means of establishing common ground, and this partly accounts for the convergence. However, there was variation in the degree to which players accommodated to each other. In the High-frequency conditions (the first and third experiments), where there was significant divergence, players not only accommodated more overall in the Competitive than in the Cooperative condition, but also accommodated more to teammates than to opponents. This strongly suggests that linguistic variants had acquired social meaning, with players associating particular variants (sometimes contextually, as in the case of Team 5a's Secret handshake in the first experiment; see Section 5.5.1) with particular players or teams, and that this influenced how players used them. From a cultural-evolutionary point of view, this can be understood in terms of selection (although, as discussed in Chapter 4 
and Section 9.5, not strictly in terms of replicator dynamics): players - whether consciously or unconsciously - selected variants on the basis of their social significance, which influenced the overall distribution of these variants.

This suggests, in line with Nettle and Dunbar (1997), Nettle (1999), Atkinson et al. (2008), and Baxter et al. (2009), that social selection should not be ignored in explanations of new-dialect formation and linguistic diversity, although it should not be assumed that it must play an important role in every case of divergence, since we should expect isolation and random drift to lead to the formation of new dialects given enough time (cf. Livingstone and Fyfe 1999; Livingstone 2002; Dunbar 2003; Trudgill 2004, 2008a). Given increasing globalisation and long-distance communication, however, few communities are now very isolated. We should expect greater contact to lower the rate of divergence though drift, and it may be that social selection will need to play an increasing role in explaining divergence in future centuries.

\subsection{Future developments}

Owing to the time and money involved in carrying out experiments with human subjects, experiments tend to be somewhat small in scale. Those described in this thesis are no exception, and a valuable future direction for the paradigm would be to investigate how larger populations of participants affect results. Nevertheless, this would undoubtedly lead experiments to take longer. A somewhat cheaper alternative (although not without its own disadvantages in terms of loss of genuine one-on-one interaction) would be an online version of the experiment that participants could engage in from their own homes, without an experimenter being present. Since it would be difficult to arrange for enough participants to be present at the same time, this would involve altering the paradigm somewhat, perhaps developing it into a more abstract, even less directly linguistic form (ongoing work presented by Dale and Lupyan 2010, involving the creation and interpretation of visual-signs for real-world objects, suggests a potential framework). A complementary approach to this would be computational simulation, which could be used to predict the results of expanding the game. 
In fact, the game can be extended in two dimensions: synchronically (or pseudosynchronically), as described above, and diachronically. Although the wordlists and cartoons given to players in the training stage of the game can be seen as modelling the output of a previous generation of speakers, the experiment was not a model of multi-generational change. It would therefore be very interesting to use the linguistic output from one game as the input for the next, to form a diffusion chain (cf. Kirby et al. 2008). The expectation is that the dialects would become more stable over time. The most significant challenge in carrying out such an experiment would lie in reducing the large amount of variation to a manageable size in such a way that there remained a meaningful connection between one generation and the next.

A further avenue to explore is in changing social dynamics. The teams in the experiment were pre-determined: players had no idea who else in the room was their teammate and had no choice in the matter; nor were they given any freedom to switch allegiance over the course of the game. It would be very interesting to introduce this freedom, particularly if players were given different dialects of the alien language at the start of the game, which, it might be expected, would lead them to disproportionately ally themselves with the players most like them linguistically.

Finally, there is the question of individual differences. As can be seen in Chapters 5 to 9 , players varied in their generosity and conservativeness. As noted in Sections 8.3.1 and 9.4, it would be interesting to investigate this further, particularly the influence this may have on accommodation rates. Players also varied in the linguistic strategies they employed, and it seems that human beings vary in the degree to which they can (or tend to) imitate other people's accents, learn second languages, and accommodate to other speakers (Giles et al. 1973; LoCastro 2001). The role of individual variation in this area, and its influence on cooperation, could be investigated experimentally either by selecting participants on the basis of their abilities or by artificially manipulating the degree to which they can accommodate.

This thesis presents not only a contribution to our understanding of how languages diverge, but also an experimental paradigm for its investigation which, it 
is hoped, will be used and adapted to contribute further to our understanding of language change and variation in the future. 



\section{ApPENDIX A}

\section{Experiment materials}

The following pages contain the instructions for each experiment (which were presented to the participants as a series of screens that they could click through at their leisure) and the feedback questionnaires (which were given to them on paper; response boxes are not included). To save space, example screenshots from the instructions will be included here for the first experiment and the Cooperative condition of the second experiment only, although they were used in all four of them.

To save space, and for ease of use, the raw data from the experiments is not included here, but is available online at: http://www.ling.ed.ac.uk/homes/gareth/ alien_language_experiment/data/ 


\section{A.1 Instructions for the experiments}

\section{A.1.1 First experiment}

\section{The game}

Welcome, and thank you for taking part. You're going to learn an alien language and use it to play a game with some other people. Please read through the following screens, which explain how it works...

You are living in a community on another planet. The whole community used to get on, but recently it's become divided into two groups of equal size. You are a member of one of these groups. Each group has a certain amount of resources available to it (like water and various kinds of food), and each member has a different amount of each resource (you may even start the game with none of a particular resource).

The object of the game is for your team to get more resources overall than the other team. You can do this by trading them, and there is a very simple rule that makes this useful:

Anything you give away is worth double to the person who receives it.

So, for example, if you give 1 point of meat to someone, they will then have 2 new points of meat. This means that exchanging resources with your own team is very good, as it increases your team's overall total. Giving resources to the other team is usually bad.

There is one other thing to bear in mind: you need to keep the resources balanced. At the end of the game, the difference between your team's highest scoring resource and the lowest scoring resource will be deducted from the score. For example, if you have 100 points of meat, less of everything else, and 0 points of something, you will lose 100 points.

Each round of the game is a few minutes long and consists of three stages. 


\section{Stage 1 - Chat}

In the first stage, you use the communicator to negotiate with another player for resources. If you don't have any water, ask them for some. They will want something in return.

But beware! The communicator is broken and will connect you randomly with another player every round. It won't tell you who you're talking to, so you don't know if the other person is on your team or not. You'll have to guess!

The decision you make is important for the next stage (which begins when the timer runs out) $\ldots$

\section{Example of stage 1}

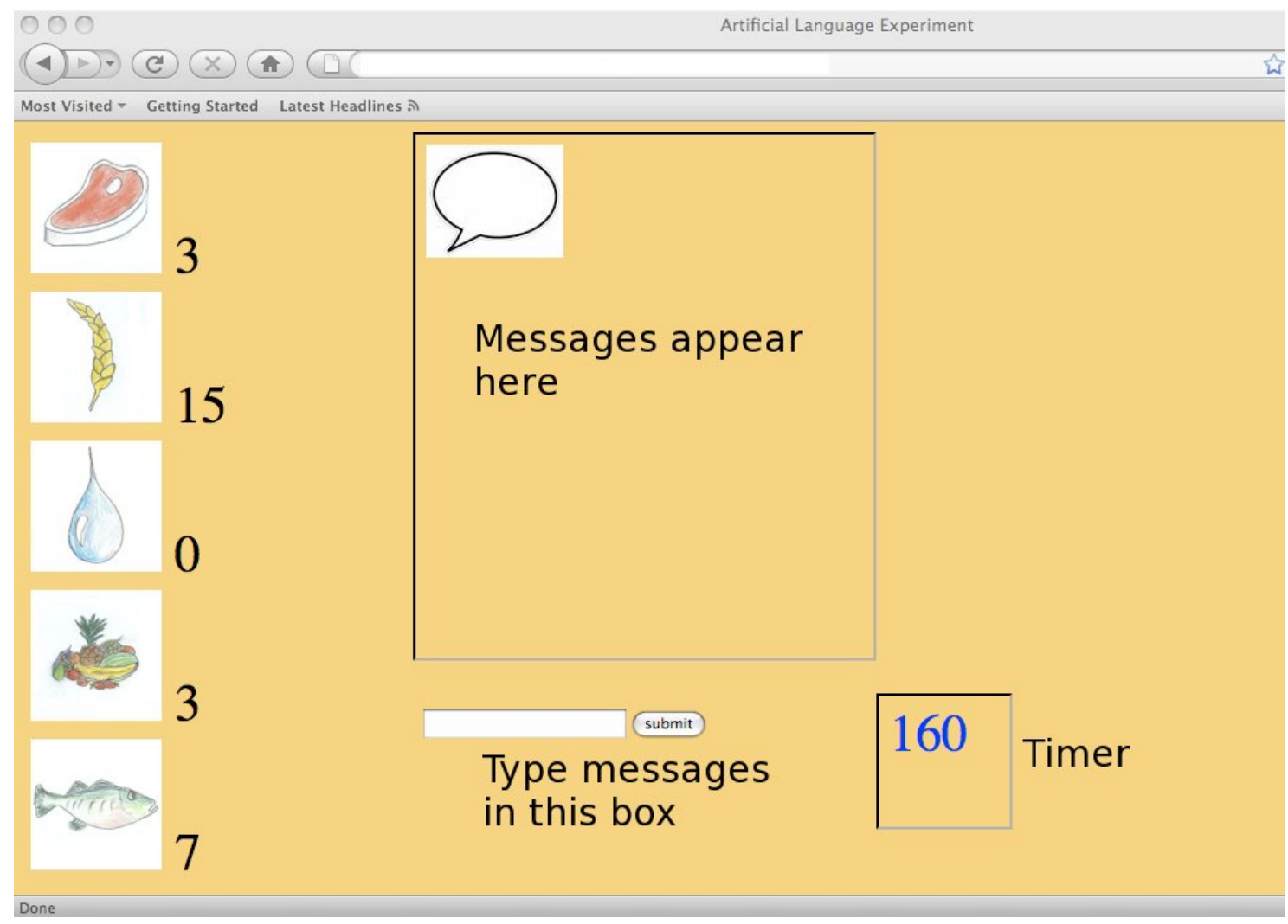




\section{Stage 2 - Exchange}

In the second stage, you can decide to give some of your resources to the player you've just spoken to. If you think they're on your team, giving them something is probably a good idea. If you think they're on the other team, it's most likely a bad idea. You're perfectly free to just sit back and not give them anything.

If you do think they're on your team and want to give them something, just click "+" next to the resource you want to give. If you make a mistake or change your mind, click"_" to take away a point.

You can give as much or as little as you like (provided you have the resources to do so). Remember that every point you give counts as double to the receiver, and to keep your resources balanced

Please also indicate how confident you are that this person is on your team. Your choice in this case does not affect the game.

When the timer runs out, you move to stage $3 \ldots$

\section{Example of stage 2}

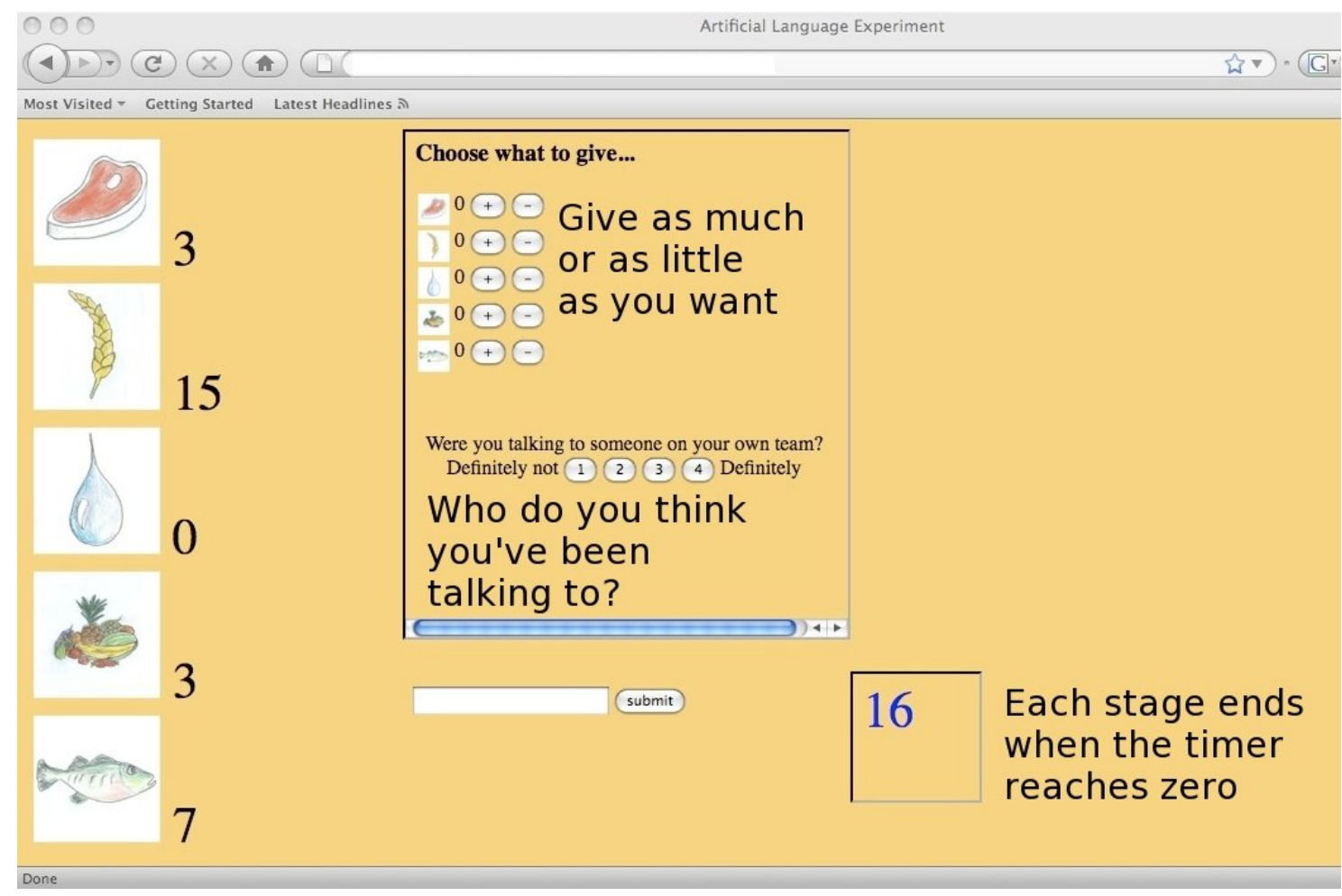




\section{Stage 3 - Feedback}

In the third stage, you finally find out if you've been dealing with a member of your own team or a member of the other one. It also reminds you if you gave them anything, and you find out if they gave you anything.

When the timer runs out, you start a new round from stage 1 . This time, the communicator might connect you with the same person again, or it might connect you with someone new.

The game carries on like this for a set number of rounds. At the end of the game, you get to see how well your team did...

\section{Example of stage 3}

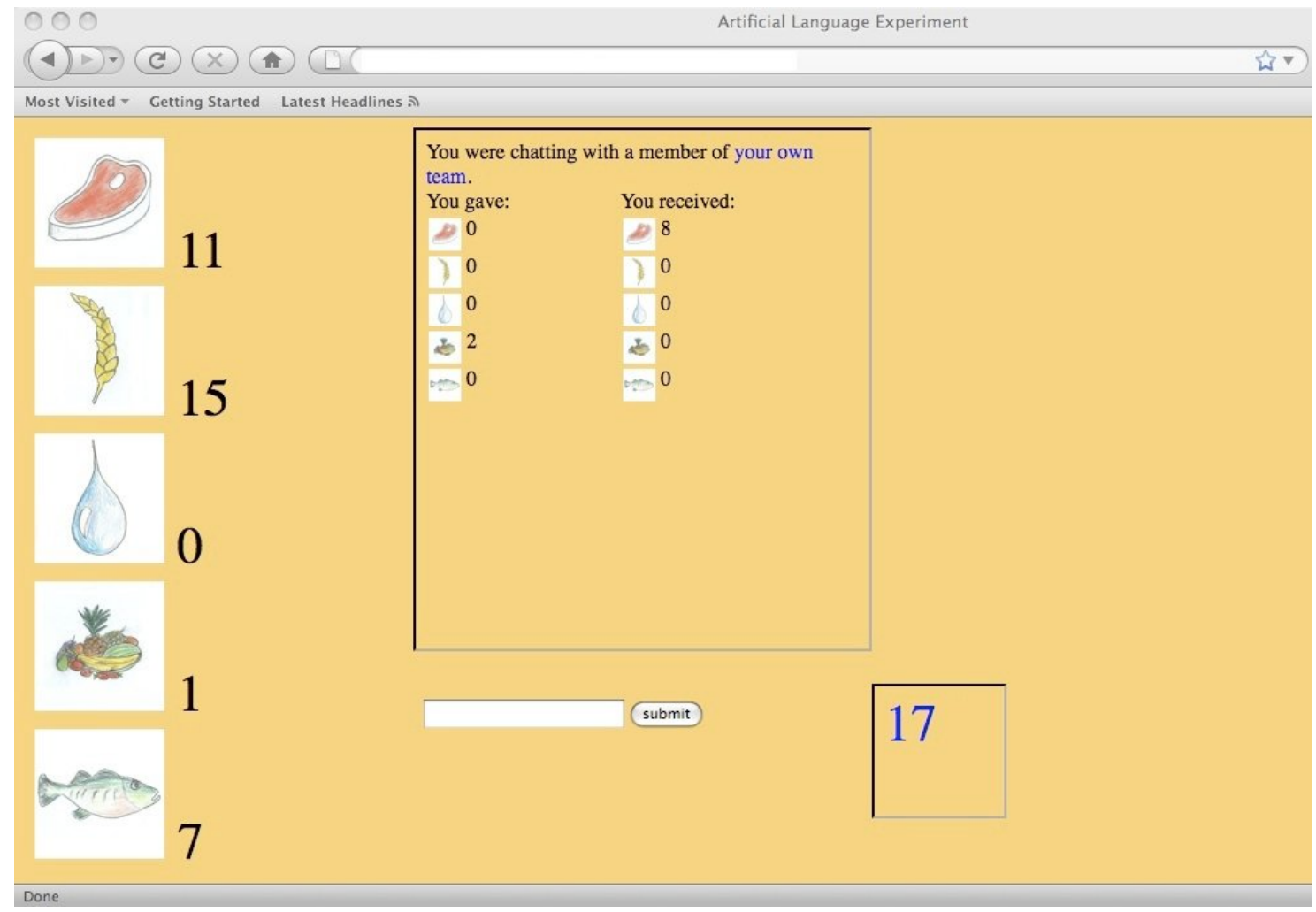




\section{Stage $4-$ Final results}

This is the end of the game, and you get to see how well your team did.

Note the penalty for unbalanced resources. The difference between your team's greatest resource and smallest resource is deducted from the total score. This is why it's important to find out what a player needs before you give them something.

\section{Example of stage 4}

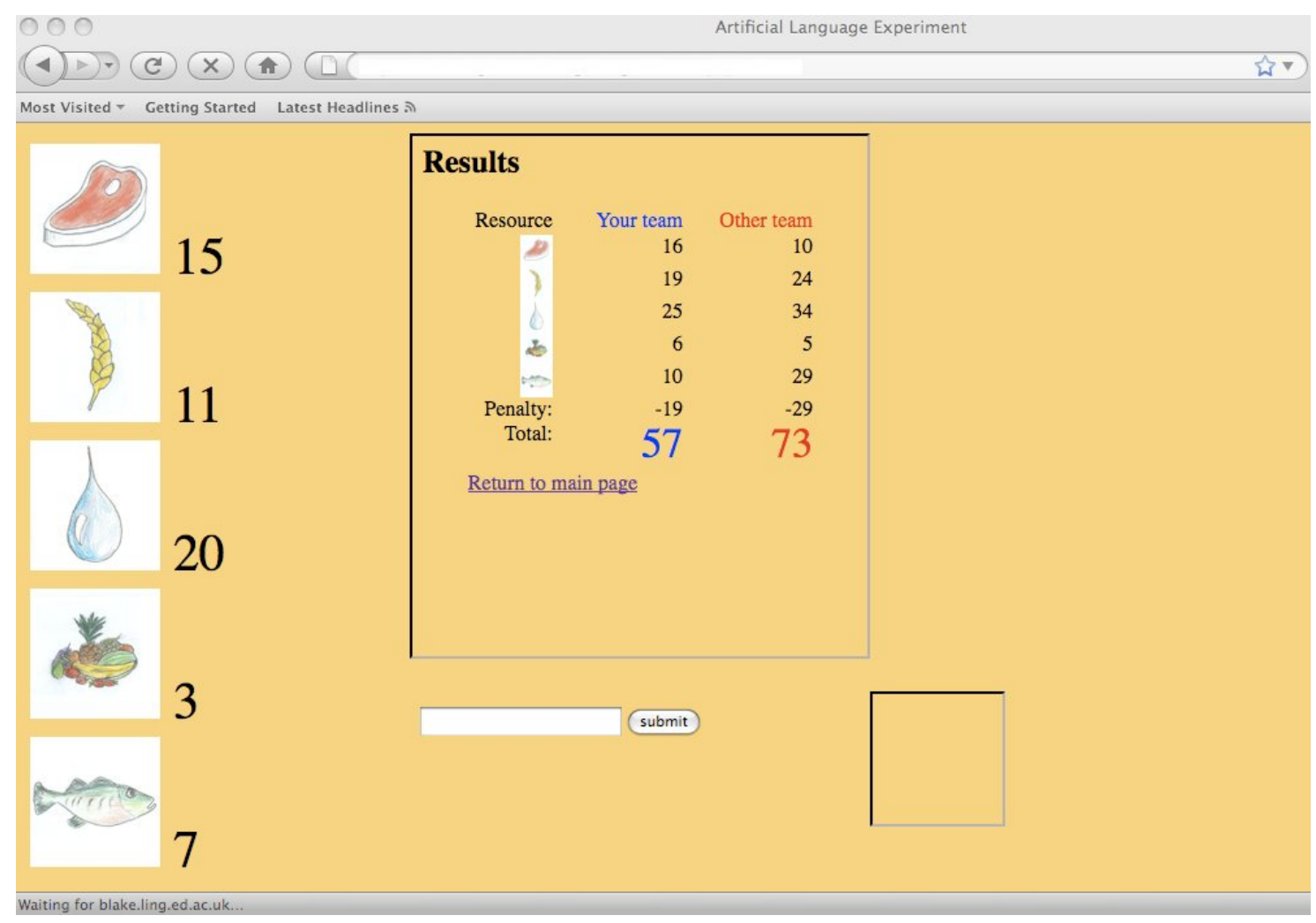




\section{Alien language}

There is a catch. You are living on an alien planet, and you have to use an alien language. You are not allowed to use English, or any other real language, in the game. This is cheating, and you'll be disqualified. You're also not allowed to use numbers.

You'll learn the alien language in a moment, then you'll play a practice round to get used to the game, and then you'll start the game properly.

Don't worry at all about making mistakes in the language. The important thing is to make yourself understood without using English (or any other real language).

And for your team to do well, of course. 


\section{A.1.2 Second experiment: Competitive condition}

\section{The game}

Welcome, and thank you for taking part. You're going to learn an alien language and use it to play a game with some other people. Here is how it works...

You are living in a community on another planet. The whole community used to get on, but recently it's become divided into two groups of equal size. You are a member of one of these groups. Each group has a certain amount of resources available to it (like water and various kinds of food), and each member has a different amount of each resource (you may even start the game with none of a particular resource).

The object of the game is for your team to get more resources overall than the other team. You can do this by trading them, and there is a very simple rule that makes this useful:

Anything you give away is worth double to the person who receives it.

So, for example, if you give 1 point of meat to someone, they will then have 2 new points of meat. This means that exchanging resources with your own team is very good, as it increases your team's overall total. Giving resources to the other team is usually bad.

There is one other thing to bear in mind: you need to keep the resources balanced. At the end of the game, the difference between your team's highest scoring resource and the lowest scoring resource will be deducted from the score. For example, if you have 100 points of meat, less of everything else, and 0 points of something, you will lose 100 points (the difference between 100 and 0 ).

Each round of the game is a few minutes long and consists of three stages. 


\section{Stage 1 - Chat}

In the first stage, you use an on-screen communicator to negotiate with another player for resources. If you don't have any water, ask them for some. They will want something in return.

But beware! The communicator is broken and will connect you randomly with another player every round. It won't tell you who you're talking to, so you don't know if the other person is on your team or not. You'll have to guess!

The decision you make is important for the next stage (which begins when the timer runs out) ...

\section{Stage 2 - Exchange}

In the second stage, you can decide to give some of your resources to the player you've just spoken to. If you think they're on your team, giving them something is probably a good idea. If you think they're on the other team, it's most likely a bad idea. You're perfectly free to just sit back and not give them anything.

If you do think they're on your team and want to give them something, just click "+" next to the resource you want to give. If you make a mistake or change your mind, click"-" to take away a point.

You can give as much or as little as you like (provided you have the resources to do so).

Please also indicate how confident you are that this person is on your team. Your choice in this case does not affect the game.

There are two things to remember:

- Every point you give counts as double to the receiver (this is how your team can accumulate points). 
- You need to keep your team's resources balanced (there is a penalty at the end if you have too much of one resource compared to others).

When the timer runs out, you move to stage $3 \ldots$

\section{Stage 3 - Feedback}

In the third stage, you finally find out if you've been dealing with a member of your own team or a member of the other one. It also reminds you if you gave them anything, and you find out if they gave you anything.

When the timer runs out, you start a new round from stage 1 . This time, the communicator might connect you with the same person again, or it might connect you with someone new.

The game carries on like this for a set number of rounds. At the end of the game, you get to see how well your team did...

\section{Stage $4-$ Final results}

This is the end of the game, and you get to see how well your team did.

Note that there is a penalty for unbalanced resources. The difference between your team's greatest resource and smallest resource is deducted from the total score. This is why it's important to find out what a player needs before you give them something. 


\section{Alien language}

There is a catch. You are living on an alien planet, and you have to use an alien language. You are not allowed to use English, or any other real language, in the game. This is cheating, and you'll be disqualified. You're also not allowed to use numbers.

You'll learn the alien language in a moment, then you'll play a practice round to get used to the game, and then you'll start the game properly.

Don't worry at all about making mistakes in the language. The important thing is to make yourself understood without using English (or any other real language).

And for your team to do well, of course. 


\section{A.1.3 Second experiment: Cooperative condition}

\section{The game}

Welcome, and thank you for taking part. You're going to learn an alien language and use it to play a game with some other people. Here is how it works...

You are living in a community on another planet. Each member of the community has a set of resources (water and various kinds of food) in different amounts (you may even start the game with none of a particular resource). The object of the game is to increase the amount of resources your community has overall. You can do this by trading resources, and there is a very simple rule that makes this useful:

\section{Anything you give away is worth double to the per- son who receives it.}

So, for example, if you give 1 point of meat to someone, they will then have 2 new points of meat.

However, there is a catch: you need to keep the resources balanced. At the end of the game, the difference between the community's highest scoring resource and the lowest scoring resource will be deducted from the score. For example, if you have 100 points of meat, less of everything else, and 0 points of something, you will lose 100 points (the difference between 100 and 0 ).

Each round of the game is a few minutes long and consists of three stages. 


\section{Stage 1 - Chat}

In the first stage, you use an on-screen communicator to negotiate with another player for resources. If you don't have any water, ask them for some. They will probably want something in return.

However, the communicator is broken and will connect you randomly with another player every round. It won't tell you who you're talking to.

\section{Example of stage 1}

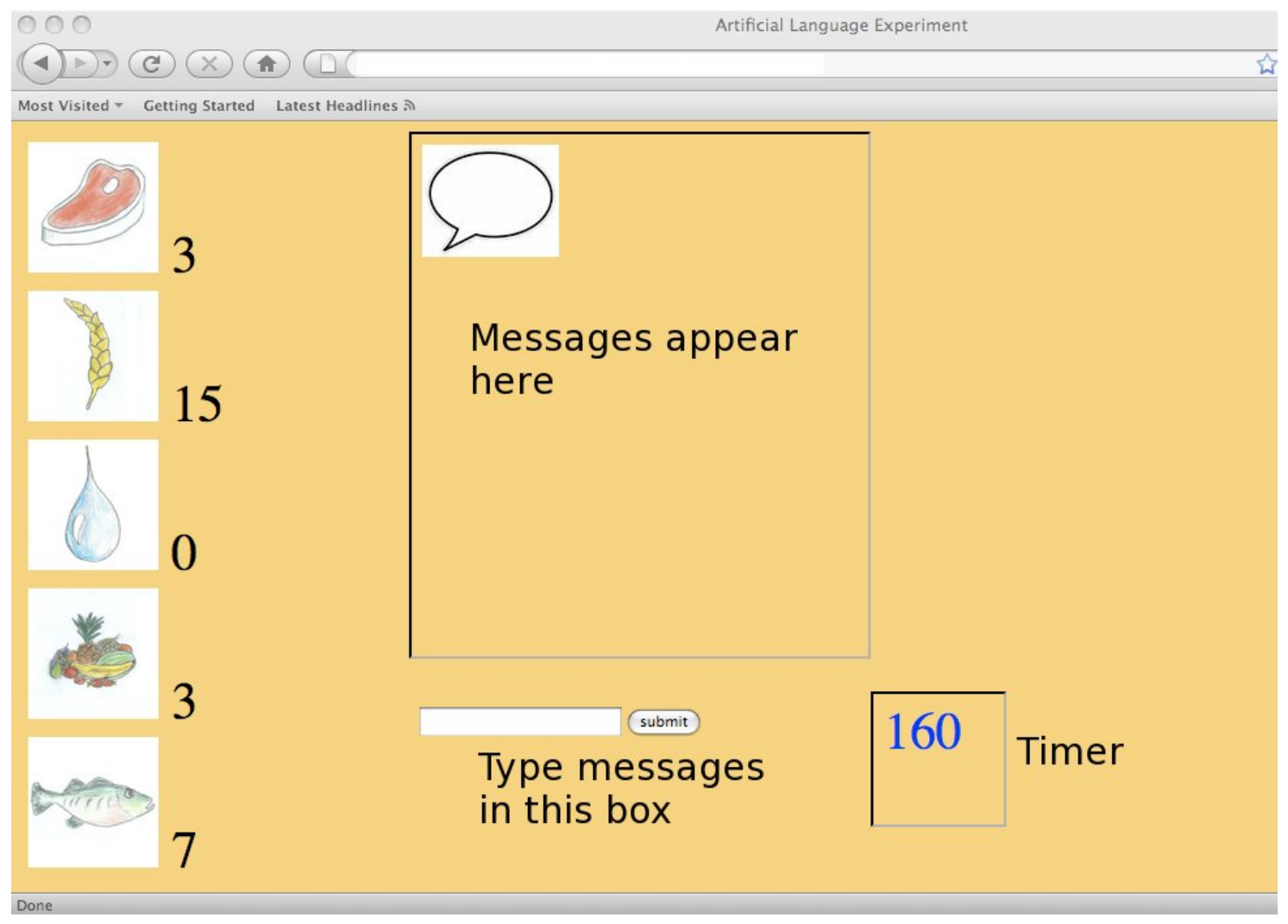




\section{Stage 2- Exchange}

In the second stage, you can decide to give some of your resources to the player you've just spoken to.

If you want to give them something, just click "+" next to the resource you want to give. If you make a mistake or change your mind, click "-" to take away a point.

You can give as much or as little as you like (provided you have the resources to do so).

There are two things to remember:

- Every point you give counts as double to the receiver (this is how the community can accumulate points).

- You need to keep your community's resources balanced (there is a penalty at the end if you have too much of one resource compared to others).

When the timer runs out, you move to stage $3 \ldots$ 
Example of stage 2

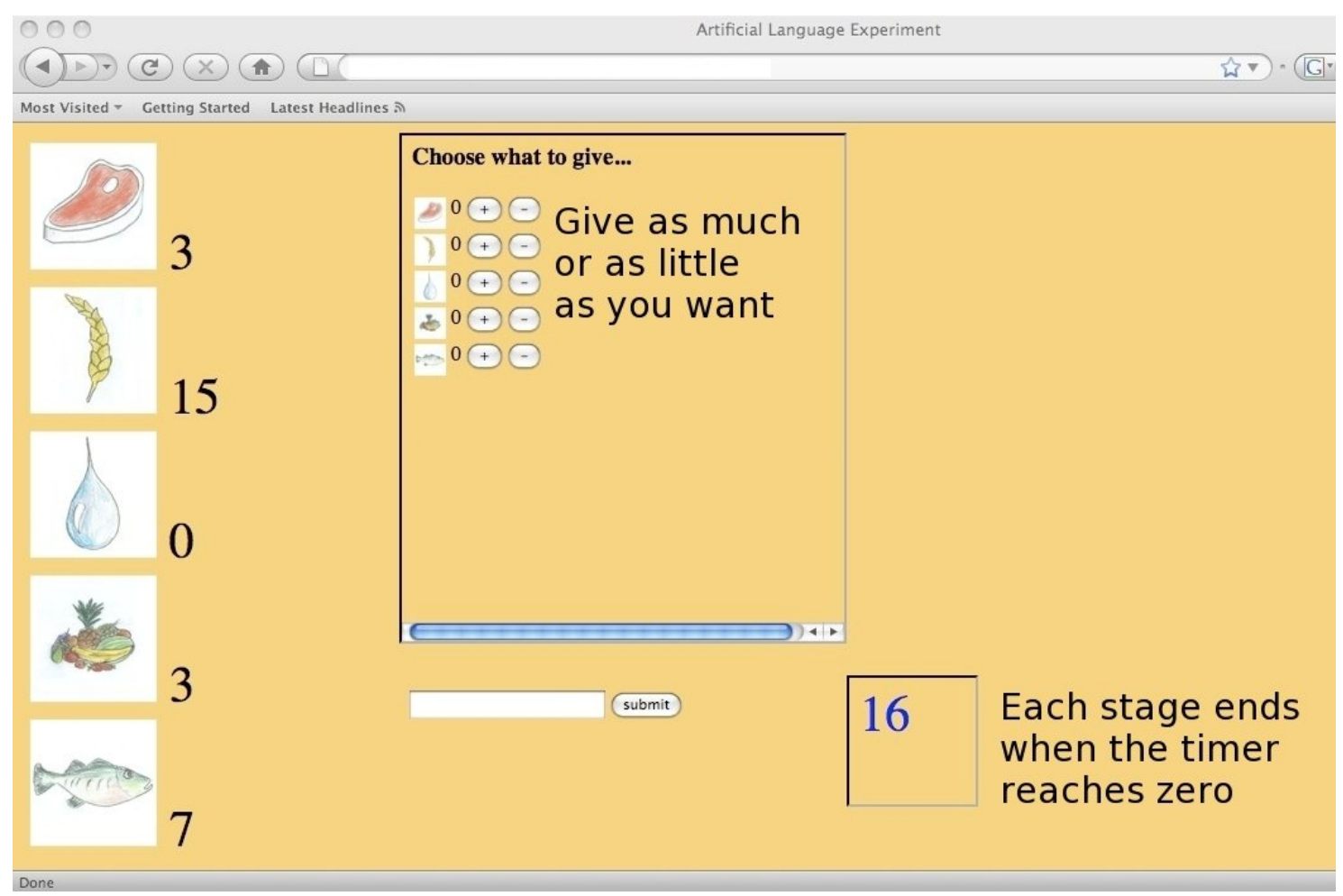




\section{Stage 3 - Feedback}

In the third stage, you are reminded what you gave the other player and you find out if they gave you anything.

When the timer runs out, you start a new round from stage 1 . This time, the communicator might connect you with the same person again, or it might connect you with someone new.

The game carries on like this for a set number of rounds. At the end of the game, you get to see how well the community did overall...

\section{Example of stage 3}

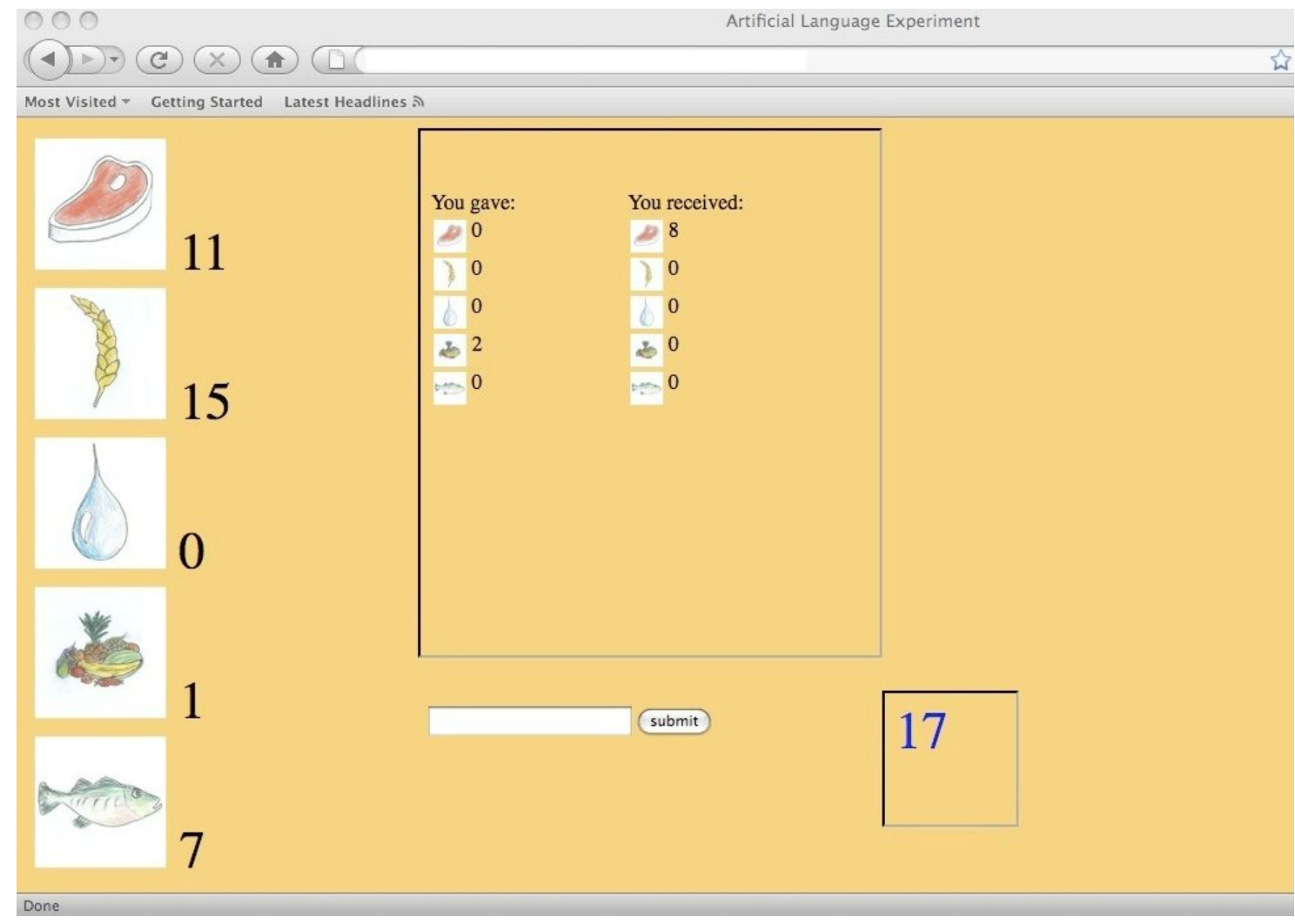




\section{Stage 4 - Final results}

This is the end of the game, and you get to see how well you did.

Note the penalty for unbalanced resources. The difference between your greatest overall resource and smallest resource is deducted from the total score. This is why it's important to find out what a player needs before you give them something.

\section{Example of stage 4}

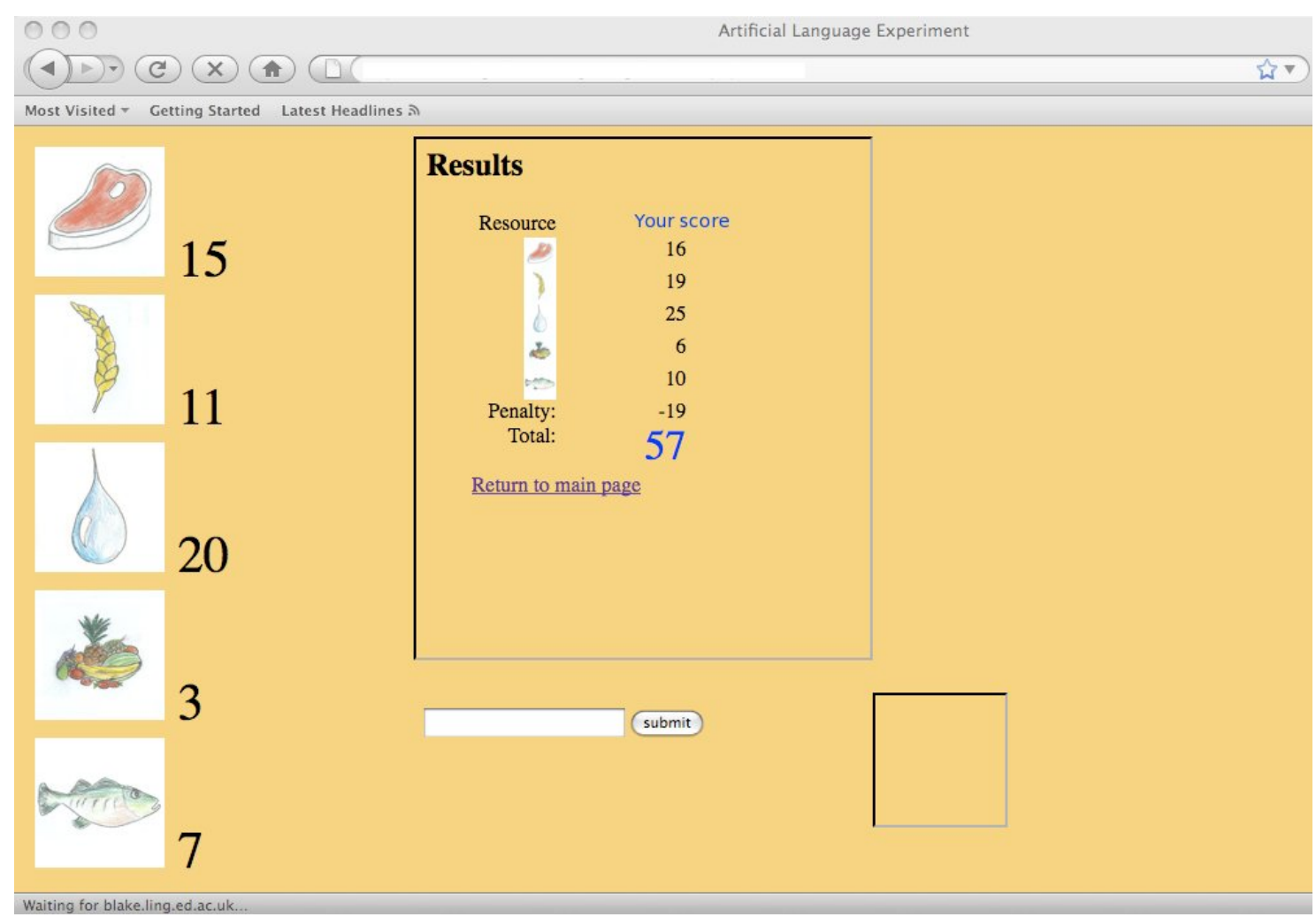




\section{Alien language}

There is a catch. You are living on an alien planet, and you have to use an alien language. You are not allowed to use English, or any other real language, in the game. This is cheating, and you'll be disqualified. You're also not allowed to use numbers.

You'll learn the alien language in a moment, then you'll play a practice round to get used to the game, and then you'll start the game properly.

Don't worry at all about making mistakes in the language. The important thing is to make yourself understood without using English (or any other real language).

And for your community to do well, of course. 


\section{A.1.4 Third and fourth experiments: Competitive condition}

\section{The game}

\section{Welcome!}

You are a member of a community of colonists on a distant planet. The whole community used to get on, but recently it's become divided into two groups of equal size. You are a member of one of these groups. Each group has a certain amount of resources available to it (like water and various kinds of food), and each member has a different amount of each resource.

Your goal is to get more resources than the other team. You can do this by trading them, and there is a very simple rule that makes this useful:

\section{Anything you give away is worth double to the per- son who receives it.}

For example, if you give 1 point of meat to someone, they will then have 2 new points of meat. This is 1 new point of meat for your whole team! This means that exchanging resources with your own team is very good. And giving resources to the other team is usually bad.

You should also try to keep your team's resources balanced. At the end of the game, the difference between your team's highest scoring resource and their lowest scoring resource will be deducted from the total score.

The game consists of a series of rounds, each of which consists of three stages. 


\section{Stage 1 - Chat}

In the first stage, you use an on-screen communicator to negotiate with another player for resources. Here you can tell the other player what you have to exchange, and what you want from them.

But beware! The communicator is broken and will connect you randomly with another player every round. It won't tell you who you're talking to, so you don't know if the other person is on your team or not. You'll have to guess!

The decision you make is important for the next stage (which begins when the timer runs out)...

\section{Stage 2 - Exchange}

In the second stage, you can decide to give some of your resources to the player you've just spoken to. If you think they're on your team, this is probably a good idea; if you think they're on the other team, this is probably a bad idea.

If you want to give something, click "+" next to the resource you want to give. If you make a mistake or change your mind, click "-" to take away a point.

You can give as much or as little as you like.

Please also indicate how confident you are that this person is on your team (your choice in this case does not affect your score)

And remember: Every point you give counts as double to the receiver (this is how your team can accumulate points).

When the timer runs out, you move to stage $3 \ldots$ 


\section{Stage 3 - Feedback}

In the third stage, you finally find out if you've been dealing with a team-mate or an opponent. You're also reminded what you gave them, and told what they gave you.

When the timer runs out, you start a new round from stage 1 . This time, the communicator might connect you with the same person again, or it might connect you with someone new.

The game carries on like this for a set number of rounds. At the end of the game, you get to see how well your team did...

\section{Stage $4-$ Final results}

This is the end of the game, and you get to see how well your team did. The score you see is for your whole team, not just you. Note the penalty for unbalanced resources. The difference between your team's greatest resource and smallest resource is deducted from the total score, so it's good to find out what your team-mate needs before you give them anything. 


\section{Alien language}

One final thing: You are not allowed to use English, or any other real language, in the game. This is cheating, and you'll be disqualified. You also can't use numbers.

Instead, you're going to use an alien language. You'll learn that in a moment; then you'll play a practice round to get used to the game; and then you'll start the game properly.

Don't worry at all about making mistakes in the language. As long as you don't use a real language, you can do what you like with the alien words. The important thing is to make yourself understood without using English (or any other real language). And for your team to do well, of course. 


\section{A.1.5 Third and fourth experiments: Cooperative condition}

\section{The game}

\section{Welcome!}

You are a member of a community of colonists on a distant planet. Each member of the community has a certain amount of resources (like water and various kinds of food), in different amounts.

Your goal is to increase the amount of resources your community has overall. You can do this by trading them, and there is a very simple rule that makes this useful:

\section{Anything you give away is worth double to the per- son who receives it.}

For example, if you give 1 point of meat to someone, they will then have 2 new points of meat. This means 1 extra point of meat for the whole community!

You should also try to keep your team's resources balanced. At the end of the game, the difference between your community's highest scoring resource and their lowest scoring resource will be deducted from the total score.

The game consists of a series of rounds, each of which consists of three stages. 


\section{Stage 1 - Chat}

In the first stage, you use an on-screen communicator to negotiate with another player for resources. Here you can tell the other player what you have to exchange, and what you want from them.

But beware! The communicator is broken and will connect you randomly with another player every round. It won't tell you who you're talking to.

\section{Stage 2 - Exchange}

In the second stage, you can decide to give some of your resources to the player you've just spoken to.

If you want to give something, click "+" next to the resource you want to give. If you make a mistake or change your mind, click "-" to take away a point.

You can give as much or as little as you like.

And remember: Every point you give counts as double to the receiver (this is how your community can accumulate points).

When the timer runs out, you move to stage $3 \ldots$

\section{Stage 3 - Feedback}

In the third stage, you're reminded what you gave the other player, and told what they gave you.

When the timer runs out, you start a new round from stage 1 . This time, the communicator might connect you with the same person again, or it might connect you with someone new.

The game carries on like this for a set number of rounds. At the end of the game, you get to see how well your community did... 


\section{Stage $4-$ Final results}

This is the end of the game, and you get to see how well your community did. The score you see is for your whole community, not just you. Note the penalty for unbalanced resources. The difference between your community's greatest resource and smallest resource is deducted from the total score, so it's good to find out what the other person needs before you give them anything.

\section{Alien language}

One final thing: You are not allowed to use English, or any other real language, in the game. This is cheating, and you'll be disqualified. You also can't use numbers.

Instead, you're going to use an alien language. You'll learn that in a moment; then you'll play a practice round to get used to the game; and then you'll start the game properly.

Don't worry at all about making mistakes in the language. As long as you don't use a real language, you can do what you like with the alien words. The important thing is to make yourself understood without using English (or any other real language). And for your community to do well, of course. 


\section{A.2 Feedback questionnaires}

\section{A.2.1 First experiment}

1. What do you think this experiment was about?

2. How well do you think you did in the game?

3. Were you able to tell if you were chatting to someone on your own team? And if so, how?

4. What did you do if you thought they were on the other team?

5. Did you ever try to trick the other team? How?

6. Did you make any deliberate changes to the alien language?

7. Please list any specific strategies you used, or tried to use, during the game, and say how successful you think they were.

8. Is there anything else you'd like to comment on? 


\section{A.2.2 Second experiment}

\section{Competitive condition}

1. What do you think this experiment was about?

2. How well do you think you did in the game?

3. Were you able to tell if you were chatting to someone on the same team as you? And if so, how?

4. What did you do if you thought they were on the same team?

5. Were you able to tell if you were chatting to someone on the other team? And if so, how?

6. What did you do if you thought they were on the other team?

7. Did you ever try to trick the other team? How?

8. Did you make any deliberate changes to the alien language?

9. Please list any specific strategies you used, or tried to use, during the game, and say how successful you think they were.

10. Is there anything else you'd like to comment on? 


\section{Cooperative condition}

1. What do you think this experiment was about?

2. How well do you think you did in the game?

3. What did you find most difficult?

4. Did you make any deliberate changes to the alien language?

5. Please list any specific strategies you used, or tried to use, during the game, and say how successful you think they were.

6. Could you ever tell (or guess) anything about who you were talking to (gender, age, identity, nationality etc.)? If so, how?

7. Is there anything else you'd like to comment on? 


\section{A.2.3 Third and fourth experiments}

\section{Competitive condition}

1. What do you think this experiment was about?

2. How well do you think you did in the game?

3. Were you able to tell if you were chatting to someone on the same team as you? And if so, how?

4. What did you do if you thought they were on the same team?

5. Were you able to tell if you were chatting to someone on the other team? And if so, how?

6. What did you do if you thought they were on the other team?

7. Did you ever try to trick the other team? How?

8. Did you make any deliberate changes to the alien language?

9. Please list any specific strategies you used, or tried to use, during the game, and say how successful you think they were.

10. Could you tell (or guess) anything about who your team-mate was (e.g. gender, age, identity, nationality etc.)? If so, how?

11. Is there anything else you'd like to comment on? 


\section{Cooperative condition}

1. What do you think this experiment was about?

2. How well do you think you did in the game?

3. What did you find most difficult?

4. Did you start to recognise other players by their use of the alien language? And if so, how?

5. Did you make any deliberate changes to the alien language?

6. Please list any specific strategies you used, or tried to use, during the game, and say how successful you think they were.

7. Could you ever tell (or guess) anything about who you were talking to (gender, age, identity, nationality etc.)? If so, how?

8. Is there anything else you'd like to comment on? 


\section{APPENDIX B}

\section{Alien language cartoons}

For the last five of the ten minutes players had to learn the alien language, they were given 12 cartoons to look at, several of which included full sentences in the language, giving them some idea of possible syntax. Figure B.1 shows how they were presented on screen. Players could click on the thumbnails to see them fullsize. The seven cartoons that were not simply pictures of the resources are shown in larger scale on the following pages.

\section{Click on the pictures to see them full-size}

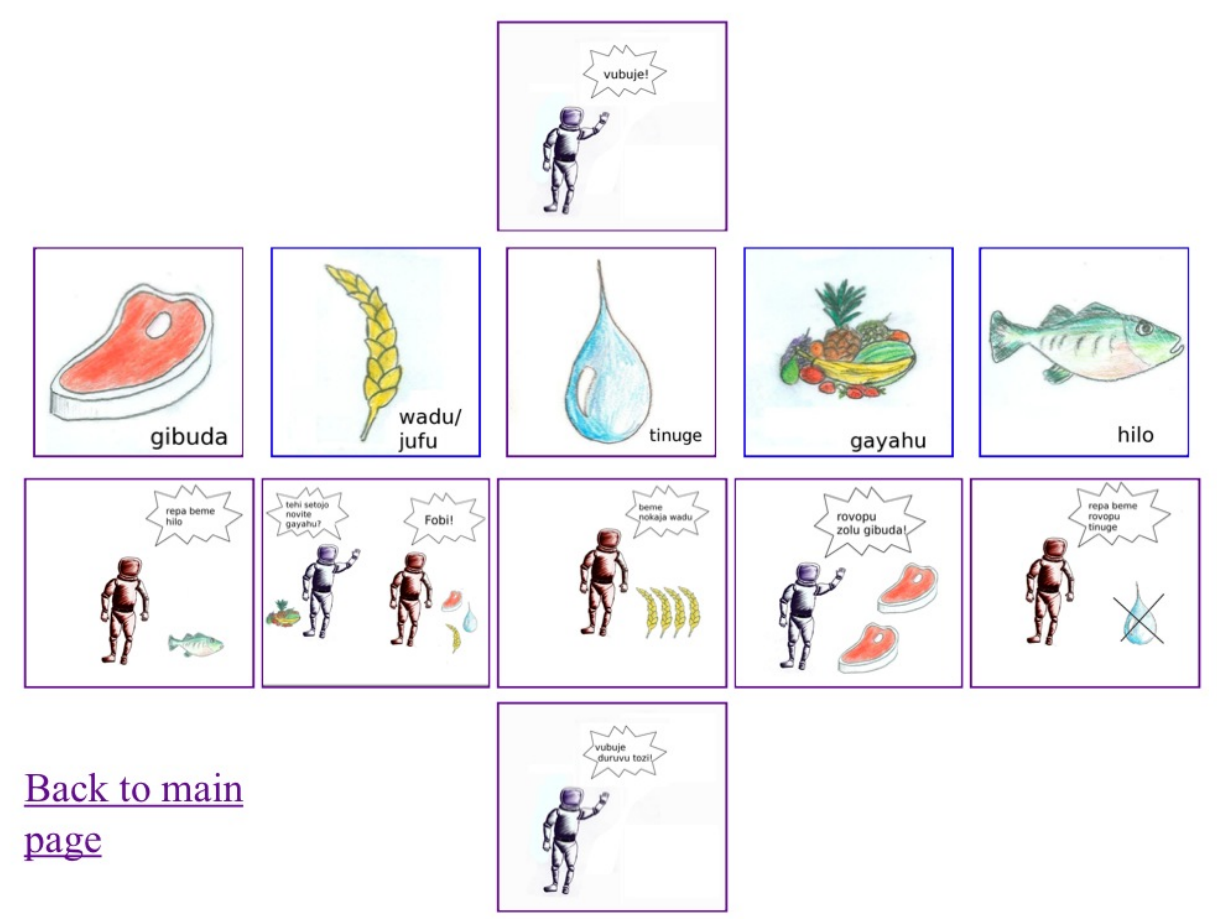

Figure B.1: Index of cartoons 

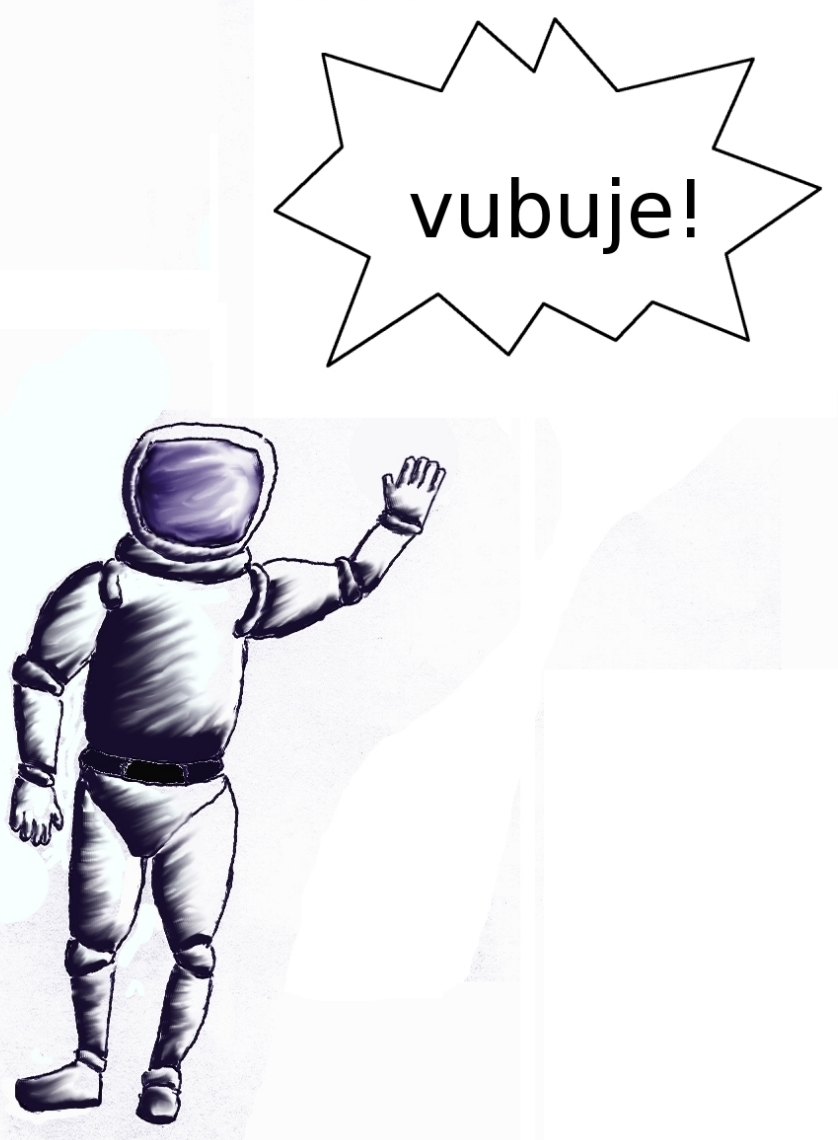

Figure B.2: Cartoon: "hello!" 

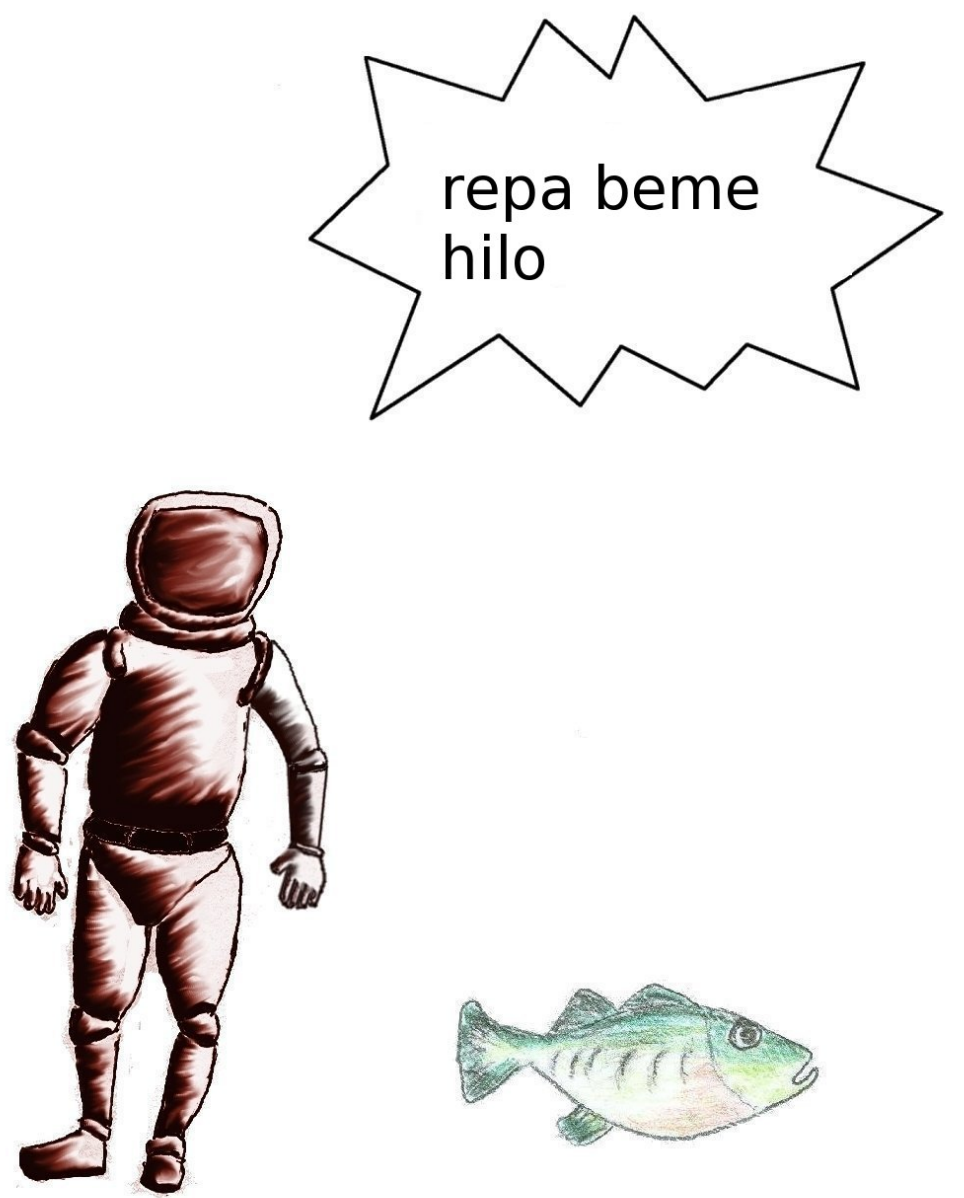

Figure B.3: Cartoon: "I have fish" 

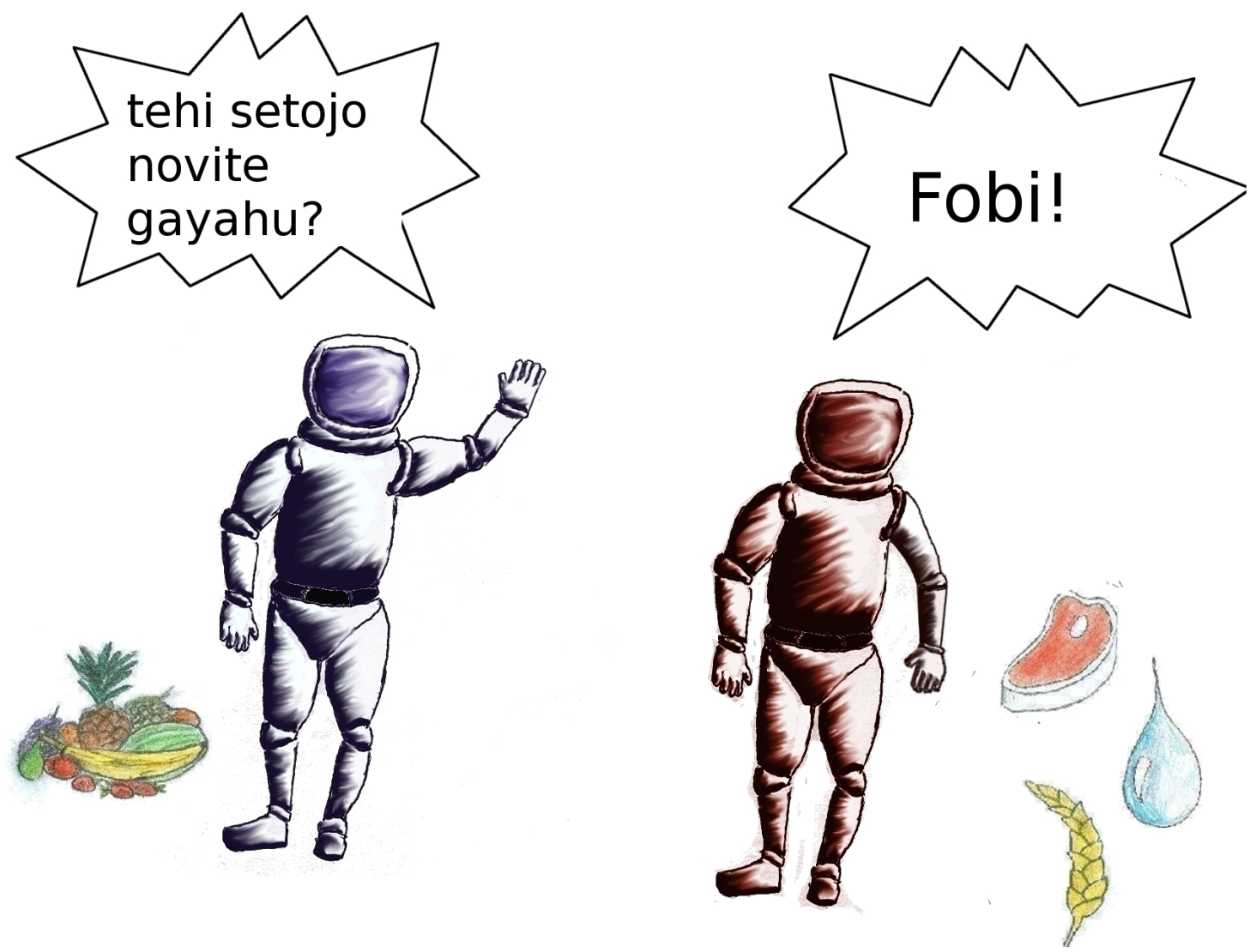

Figure B.4: Cartoon: "do you want fruit?" "Yes!" 


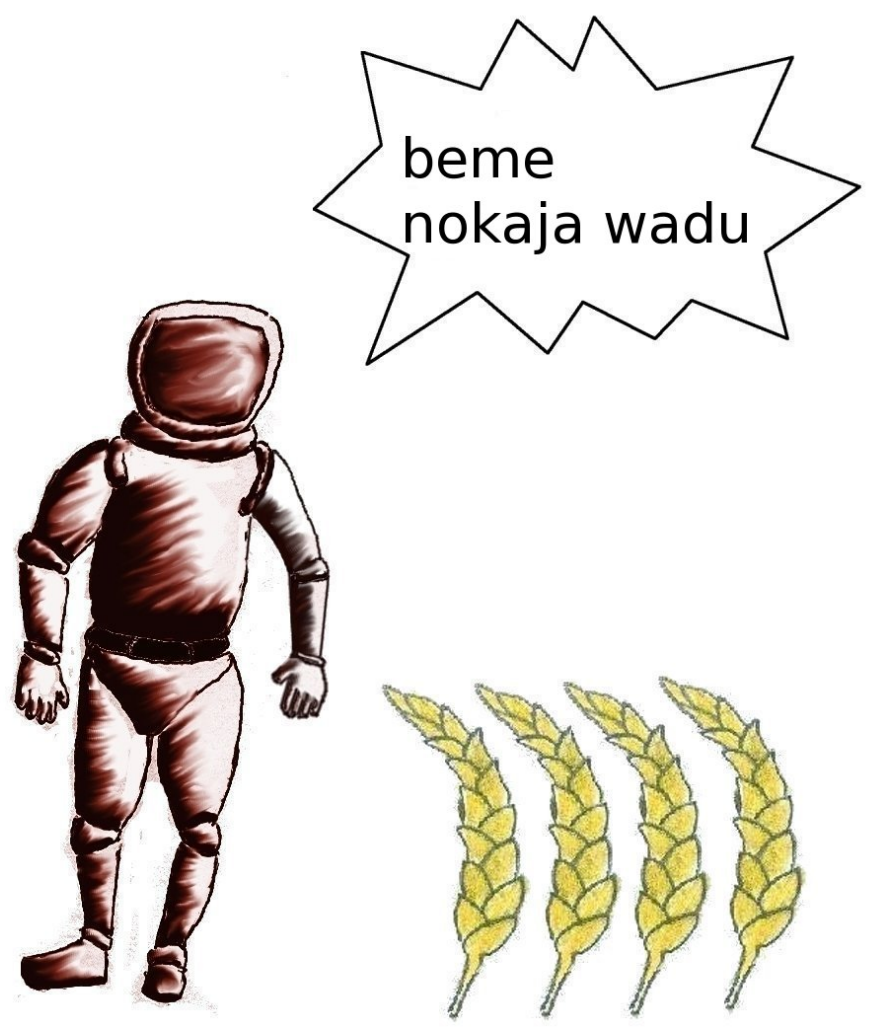

Figure B.5: Cartoon: "have much corn" 

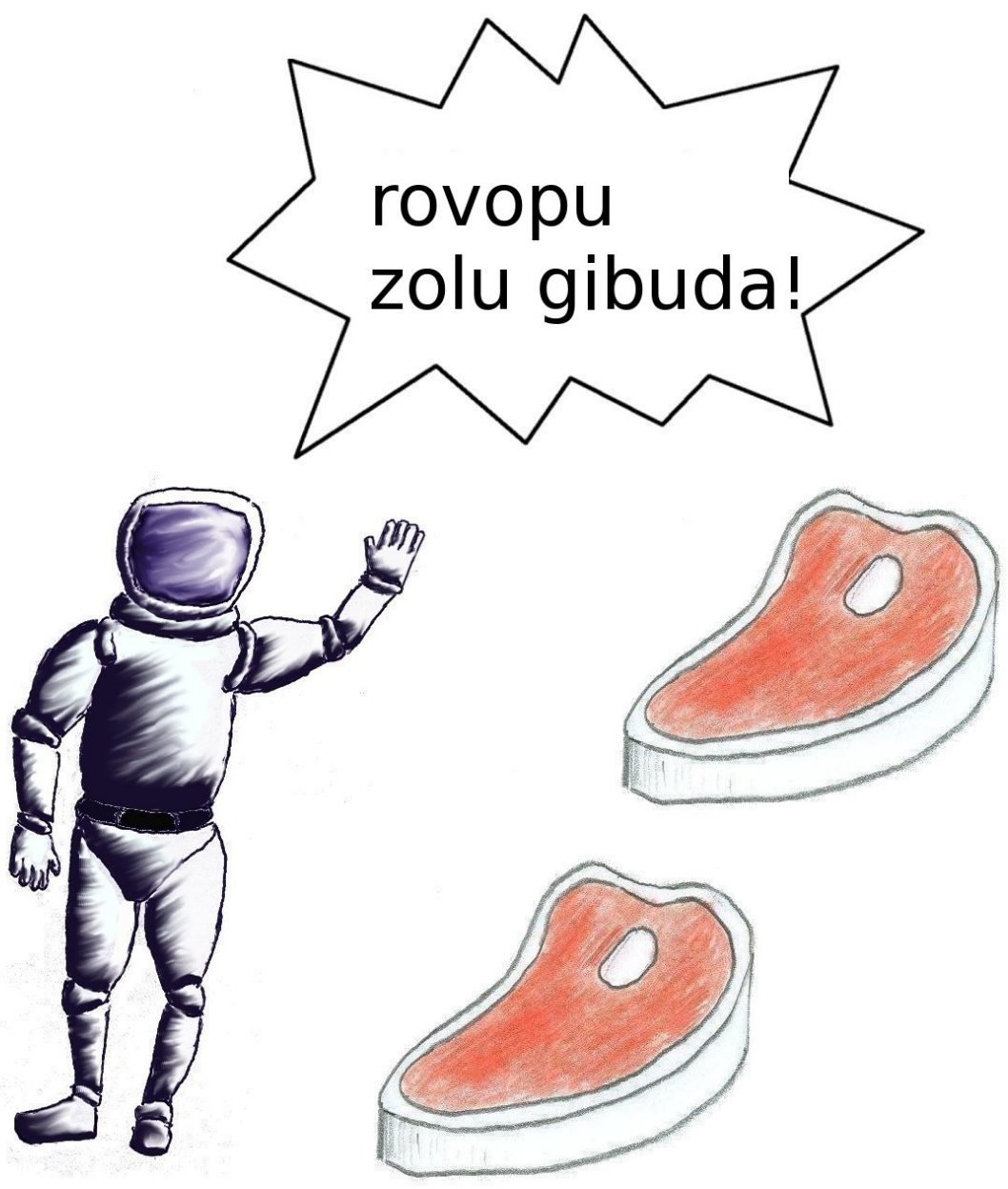

Figure B.6: Cartoon: "no need meat!" 


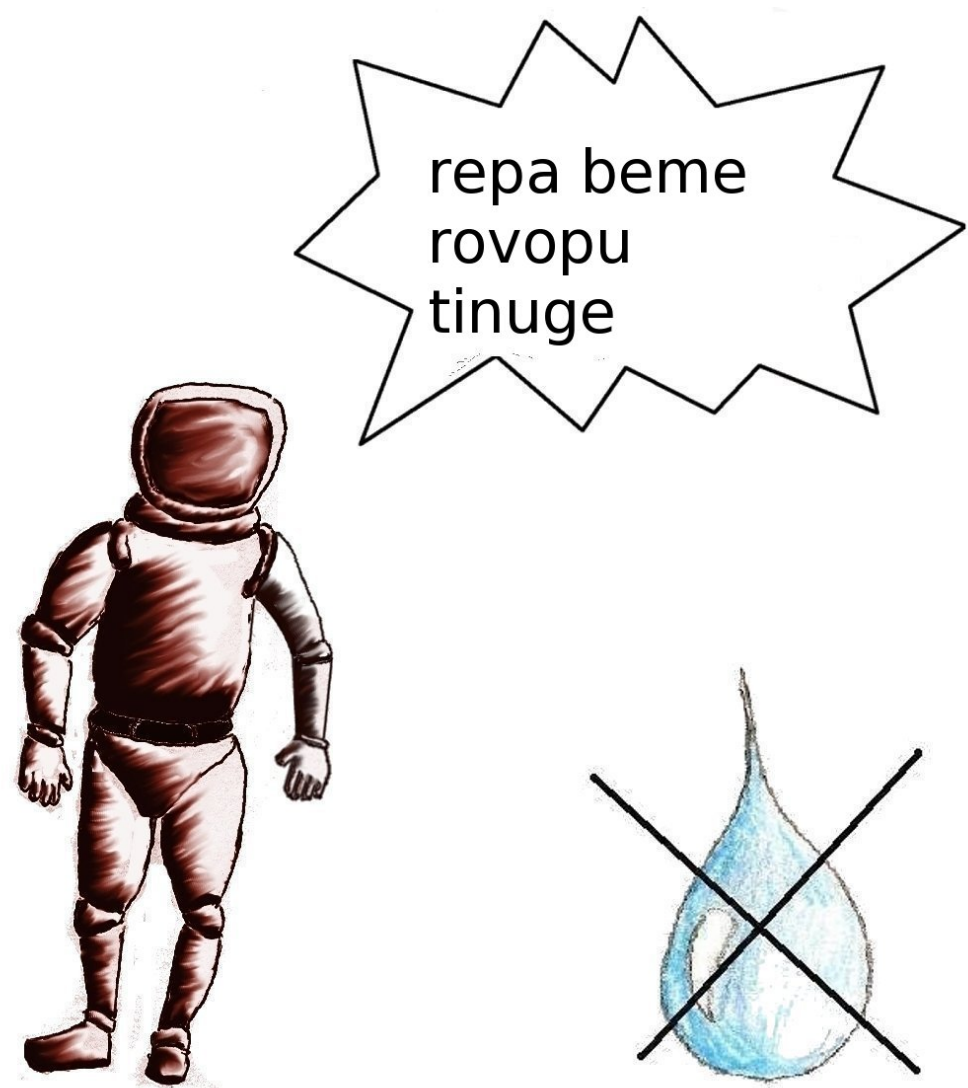

Figure B.7: Cartoon: "I have no water" 

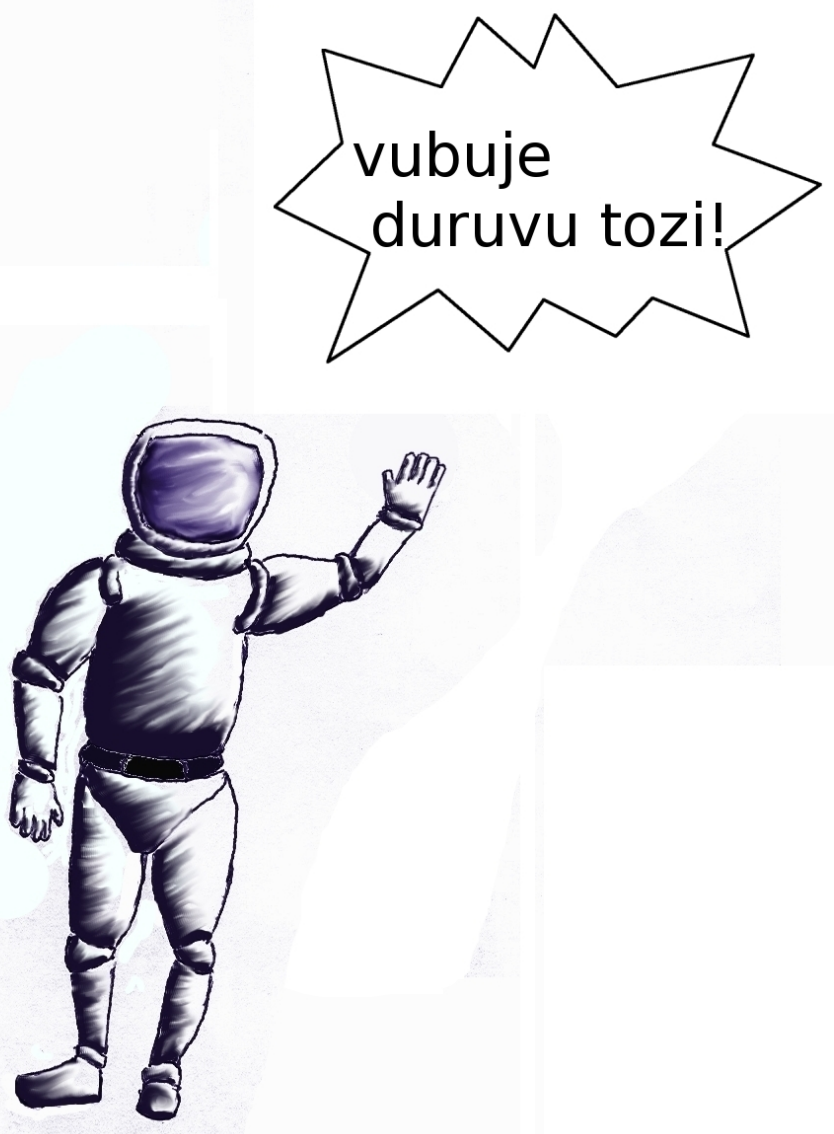

Figure B.8: Cartoon: "goodbye and thanks!" 


\section{APPENDIX C}

\section{Calculating divergence}

The divergence reported in this thesis is based on a comparison of intra-team and inter-team convergence. A condition showing significant divergence, therefore, is a condition in which players are significantly more like their teammates than their opponents in their use of the alien language.

Players were compared on the basis of variable sets. A variable set is a set of all the word-forms used in a particular game with a particular meaning, together with a frequency count for every player. The example from the first experiment (see page 92) is repeated below (Table C.1), where the number in brackets represents the proportional frequency: that is, the raw frequency count for that word divided by the sum of frequencies for that player in that set. Player 3, for example, used three different words to express the meaning "want/need". The frequency counts for these three words is 21 , so the proportion for the form rohapi, which was used 11 times, is $11 / 21=0.52$.

\begin{tabular}{lllll}
\hline & \multicolumn{2}{c}{ Team 1 } & \multicolumn{2}{c}{ Team 2 } \\
& Player 1 & Player 2 & Player 3 & Player 4 \\
\hline rohapi & $34(.97)$ & $27(.93)$ & $11(.52)$ & $10(.48)$ \\
roahpi & $1(.03)$ & $2(.07)$ & $0(0)$ & $0(0)$ \\
ropahi & $0(0)$ & $0(0)$ & $9(.43)$ & $9(.43)$ \\
rophui & $0(0)$ & $0(0)$ & $1(.05)$ & $0(0)$ \\
wohapi & $0(0)$ & $0(0)$ & $0(0)$ & $2(.09)$ \\
\hline
\end{tabular}

Table C.1: Example variable set from first experiment 


\begin{tabular}{lllll}
\hline & \multicolumn{2}{c}{ Team 1 } & \multicolumn{2}{c}{ Team 2 } \\
& Player 1 & Player 2 & Player 3 & Player 4 \\
\hline yaji & $23(.82)$ & $0(0)$ & $0(0)$ & $0(0)$ \\
jadi & $0(0)$ & $17(.37)$ & $0(0)$ & $0(0)$ \\
yadi & $0(0)$ & $25(.54)$ & $0(0)$ & $0(0)$ \\
yagi & $5(.18)$ & $4(.09)$ & $69(1)$ & $56(1)$ \\
\hline
\end{tabular}

Table C.2: Example variable set scoring 0.5

\begin{tabular}{lllll}
\hline & \multicolumn{3}{c}{ Team 1 } & \multicolumn{2}{c}{ Team 2 } \\
& Player 1 & Player 2 & Player 3 & Player 4 \\
\hline Player 1 & 0 & .455 & $\mathbf{. 4 1}$ & $\mathbf{. 4 1}$ \\
Player 2 & .455 & 0 & .455 & .455 \\
Player 3 &. $\mathbf{4 1}$ & .455 & 0 & $\mathbf{0}$ \\
Player 4 & $\mathbf{. 4 1}$ & .455 & $\mathbf{0}$ & 0 \\
\hline
\end{tabular}

Table C.3: Mean distances from example set in Table C.2

For every condition of the experiment, all the variable sets were gathered together and scored. This score was based on the mean Euclidean distance between players (that is, between the proportional frequency counts, rather than the raw counts). If every player in a given set was closer to their teammate than to either opponent, the set was scored 1 (as was the case for the set in Table C.1). If this was true of both members of one team, but not the other, then the set was scored 0.5 (see Tables C.2 and C.3). Otherwise the set was scored 0. The sum of these scores gave an overall divergence score for each condition. To test whether or not this overall score was significant, a Monte Carlo test was carried out. This meant shuffling all the raw frequency counts, recalculating the proportions, and rescoring all sets thousands of times (in fact every dataset was shuffled and rescored 10,000 times, a figure that balanced reliability with practicality). ${ }^{1}$ In fact there were three ways in which the data could have been shuffled:

1. Any frequency score can appear anywhere else in the entire dataset;

2. Any frequency score can appear anywhere else in the same variable set;

3. Any frequency score can appear anywhere else in the same row.

\footnotetext{
${ }^{1}$ See Edgington (1995) for a good introduction to randomisation tests, including arguments in favour of their wider use on the basis of their transparency, applicability, and versatility - they can be tailored to a very broad range of problems.
} 
There are advantages and disadvantages to each of these. The first is the simplest. However, information about the structure of variable sets is lost with this method: in every set, certain variants are particularly favoured, and others (such as the results of occasional typing errors) not. This is an important fact that is not captured in the first model. The third maintains this structure, but there is a danger here that the results might be skewed by data from one game. The second measure is an unhappy compromise, since it has both the disadvantages, but neither of the advantages, of the first and third methods. As it happens, the dilemma of which to choose need not concern us too much: all three methods produce similar results (see Tables C.4 and C.5). Results reported in the main body of the thesis are based on the third method, since this is the method with which the least information is lost.

\begin{tabular}{llll}
\hline & $\begin{array}{l}\text { First } \\
\text { method }\end{array}$ & $\begin{array}{l}\text { Second } \\
\text { method }\end{array}$ & $\begin{array}{l}\text { Third } \\
\text { method }\end{array}$ \\
\hline First experiment & $0^{* * *}$ & $0^{* * *}$ & $0^{* * *}$ \\
Second experiment & .827 & .887 & .566 \\
Third experiment & $.006^{* *}$ & $.023^{*}$ & $.001^{* *}$ \\
Fourth experiment & .43 & .644 & .364 \\
\hline
\end{tabular}

Table C.4: P-values for divergence depending on shuffling method (Competitive conditions)

\begin{tabular}{llll}
\hline & $\begin{array}{l}\text { First } \\
\text { method }\end{array}$ & $\begin{array}{l}\text { Second } \\
\text { method }\end{array}$ & $\begin{array}{l}\text { Third } \\
\text { method }\end{array}$ \\
\hline Second experiment & 1 & .999 & .937 \\
Third experiment & .447 & .616 & .154 \\
Fourth experiment & .579 & .578 & .306 \\
\hline
\end{tabular}

Table C.5: P-values for divergence depending on shuffling method (Cooperative conditions) 



\title{
APPENDix D
}

\author{
Published papers
}

This appendix includes two papers published before completion of the thesis: Roberts (2008) and Roberts (2010). 


\title{
Language and the freerider problem: an experimental paradigm
}

\author{
Gareth Roberts \\ Language Evolution and Computation Research Unit, \\ University of Edinburgh \\ gareth@ling.ed.ac.uk
}

\begin{abstract}
Change and variation, while inherent to language, might be seen as running counter to human communicative needs. However, variation also gives language the power to convey reliable indexical information about the speaker. This has been argued to play a significant role in allowing the establishment of large communities based on cooperative exchange (Enquist and Leimar 1993; Dunbar 1996). There has, however, been little experimental investigation of this hypothesis. This paper presents a preliminary study intended to help fill this gap. Participants played a team-game online in which they negotiated anonymously for resources using an artificial language. Players succeeded in using linguistic cues to distinguish between their team-mates and their opponents and this led to the development of between-team variation in the use of the language.
\end{abstract}

Keywords: Language evolution; Language change; Freerider problem; Historical linguistics; Sociolinguistics; Cultural evolution; Experimental 


\section{DRAFT COPY - PLEASE DO NOT CITE WITHOUT PERMISSION OF AUTHOR}

Much recent work has recognised that language is an adaptive system in its own right (see e.g. Deacon 1997/1998, Steels 2000, Kirby and Hurford 2002, Christiansen and Chater 2007). This has deep roots - the image of language as a living thing was popular among eighteenth- and nineteenth-century linguists, who took it in some cases to fanciful extremes (such as Jacob Grimm's Sprachgeist). In modern evolutionary linguistics, however, it represents a change of focus: rather than assuming that language is highly genetically specified and focussing attention on the selective pressures that led to this, the cultural evolutionary standpoint shifts some of the burden of explaining linguistic structure onto the pressures acting on language itself. For example, in acquiring a language, an infant is exposed to only a small part of it. This has traditionally raised questions as to the cognitive mechanisms involved in forming correct generalisations about the language. From a cultural evolutionary viewpoint, however, the "transmission bottleneck" (for an early use of this term see Deacon: 110) helps explain something about its structure. Language is forced to change to be learnable; low-frequency irregularities disappear (cf. Lieberman et al. 2007; Pagel et al. 2007); and systematicity increases (Kirby 2000; Cornish 2006). This contributes to the appearance of design.

Language, however, changes rather more than good design would appear to dictate. As Labov (2001: 6) puts it:

Linguists and language teachers get some employment from the results of language divergence. But that seems to be about all the benefit there is to language change. It is hard to avoid the conclusion that language, as an instrument of communication, would work best if it did not change at all.

To some extent this is just the way it has to be: in any cultural system, drift is inevitable. In addition, we should not expect the pressures acting on language to be equal in all cases. Various factors, like the invention of writing, or the degree to which a language is used for intragroup versus inter-group communication, can be expected to have a significant impact (Wray and Grace 2007). But this leaves unanswered the question of why language should not be more genetically specified in the first place. It would seemingly be of enormous advantage to be able to communicate instantly with conspecifics without the need for interpreters. Yet, while a certain amount of the structure of language may be hardwired, so much of language is culturally transmitted that dialects only a few miles apart can diverge sufficiently in a relatively short time to cause serious communication difficulties. There are several factors involved here. One is the time available: there may simply not have been enough for greater hardwiring to have evolved (for a proposed "speed limit" in biological evolution, see Worden 1995). Moreover, it should not be assumed that, given enough time, such hardwiring would occur: as Tooby and Cosmides (1990: 21) point out, the "evolutionary process can be said to store information necessary for development in both the environment and the genes." Indeed, the speed of cultural evolution relative to biological evolution means that language can rapidly adapt to a changing environment: something that is of great benefit to its speakers (cf. Worden: 148). It is also not clear how much benefit there would be to an individual whose language was much more genetically specified. There has recently been evidence that combinations of certain alleles may bias a population towards speaking, or not speaking, a tonal language (Dediu and Ladd 2007). However, this is a case of genes being selected for reasons almost certainly unrelated to language; the linguistic bias (if that is indeed what explains the correlation) is an accidental by-product. It is, moreover, only a small bias, whose effects are felt only after many generations. Importantly, any cognitively normal human infant can acquire any human language, be it tonal or non-tonal. It is hard to see why a stronger genetic bias, strong enough to noticeably influence the speech patterns of a particular individual, would be selected for, unless linked to some other beneficial phenotypic trait. Or what of an English-biased mutant born into an English-speaking community? The only 
obvious benefit would be an ability to acquire the language faster. Yet this benefit would have to be great enough for the allele to spread through the population before the speech patterns of that population changed to the point that it was no longer beneficial.

There is something else involved. Variation and change are useful to us. Any speaker of a language knows that not everyone pronounces and uses words in quite the same way; any speaker also knows that such variation has great social significance. Labov claims that an unchanging language would be a better "instrument of communication", but all utterances communicate more than the sum meaning of their words and syntax. We all belong to various social groups, based on origin, profession, class and so on; and when we speak, we transmit information about our membership of such groups. This would be impossible without variation, the result of change. It is only because Britons and Americans tend to speak differently that the concept of an "American accent" is meaningful. ${ }^{1}$ Significantly, this kind of indexical ${ }^{2}$ information is a good deal more reliable than the propositional kind: any English speaker can say, "I was born and raised in Australia." Only a minority can say it in such a way that an Australian is likely to believe them immediately.

\section{Markers}

It is hardly disputed that human beings exploit the variation inherent in language and employ it as a marker; but to what end? There is good reason to suppose that this ability to signal one's membership of a group (however that may be defined), and to recognise others' membership, has been vital in allowing the foundation and maintenance of communities based on cooperative exchange. $^{3}$ Reciprocal altruism relies on the ability to detect and deal with "freeriders", or defectors, who exploit the cooperative behaviour of others. Punishment and reputation play a crucial role in minimising the effect of such behaviour on the part of other community members (Axelrod 1984/1990: 150-4; Boyd et al. 2003). Even here, language plays an important role, as a means of reporting who did what to whom (Dunbar 1996). Reputation and gossip, however, are of little use in dealing with freeriders from outside the community, who may not stick around long enough to face the consequences (Enquist and Leimar 1993). An obvious tactic on the part of the community members in this case is to act altruistically only towards other members of the group. As one's group increases in size, however, distinguishing between insiders and outsiders becomes very difficult. Some kind of shared marker is a prerequisite for the establishment of a large cooperative network.

The ideal marker must be costly to obtain and hard to imitate; otherwise it is unreliable. However, it must also be sufficiently flexible to cope with inevitable changes in the structure of communities, and sufficiently salient to function usefully as a marker. Genetically encoded markers may fulfil the first two criteria well, but they are highly inflexible. Cultural markers like clothing and tattoos are more flexible, but easier to imitate. By comparison, the patterns of an individual's speech are extremely well suited to this purpose ${ }^{4}$ : they are salient, flexible and close to inalienable. They are acquired young and are hard to change in adulthood. Indeed, the only reliable means of acquiring them is through prolonged exposure. Moreover, people are capable of multiple competencies: at least if we are exposed to them early enough, we can all acquire more than one set of speech patterns and switch between them according to context.

\footnotetext{
${ }^{1}$ Of course, the term "American accent" actually stands for a multitude of accents, based on narrower geographical definitions, social divisions and so on.

${ }^{2}$ I use the term not in the Piercian sense, contrasted with iconicity and symbolicity, but in the sociolinguistic sense, in which a particular linguistic feature indexes something with social meaning.

${ }^{3}$ One might speculate that it played a more important role, earlier in our evolutionary history, in identifying kin.

${ }^{4}$ This is not to say that the other things mentioned are never used as markers of group identity in the context described. They all almost certainly are, along with other things. The point is that language does the job especially well.
} 
Several claims are being made here:

- To distinguish between insiders and outsiders to their group, human beings exploit variation in cultural (and biological) systems.

- We have evolved to be highly adept at spotting and interpreting the cues provided by such variation.

- This helps us to maintain social networks based on cooperative exchange.

- This has an effect on the evolution of cultural systems (and, potentially, biological traits).

- Language is especially well suited to this purpose; it is, indeed, the cultural system best suited to it.

In cases where there is pressure to distinguish between insider and outsider, we should therefore expect human beings to be adept at using linguistic cues to make such distinctions; we should further expect this to have a linguistic effect, specifically divergence into different dialects (as well, potentially, as the apparently superfluous retention of irregularities and "difficult" features that might distinguish non-natives from natives; cf. Wray and Grace 2007).

It is important to note what claims are not being made: first, although this may be a trigger for linguistic change, and potentially an important one, it should not be assumed to be the only one; other factors - like analogy, phonetic reduction, misanalysis, and so on - are clearly involved. The degree to which human beings actually innovate linguistically for the sake of creating a marker (as opposed to selecting a particular variant that already exists) is an empirical question that cannot be answered a priori. Second, the use of language in this way cannot be used to explain the existence of a critical or sensitive period for language acquisition. It should not be beneficial to an individual to be less able to acquire new patterns in adulthood.

\section{Empirical Investigation}

The indexical role of variation has long been recognised by linguists. Labov's (1963) Martha's Vineyard study (which can reasonably be said to be a foundational paper in the discipline of sociolinguistics) showed how speakers' choice of a phonetic variant correlated well with their attitude towards outsiders. Related themes can be found in later sociolinguistic work. The concepts of prestige and covert prestige (see e.g. Trudgill 1972), where speakers aim to associate themselves linguistically with a particular socially defined group, are clearly relevant. More recently, Evans (2004) showed how gender and integration into the local community were significant predictors of the extent to which migrants to Ypsilanti, Michigan acquired a feature of the local accent. The role of gender is interesting and echoes Labov's three generalisations about gender and language variation (see Labov 1990, 2001), which make women the leaders of linguistic change. One might speculate that this reflects a tendency towards female exogamy, voluntary or otherwise, in early human societies (Hagen 2008 notes that women of reproductive age are disproportionately lacking among the remains of the dead at the site of an ancient massacre), making it more important for women to be able to integrate well with new communities in adulthood. But this is clearly not the only plausible explanation.

Sociolinguistic studies of existing speech communities are clearly very valuable to our understanding of linguistic variation and change. However, and particularly with regard to research into the evolution of language, they are also limited by the kind of communities that actually exist and are available for investigation. For ethical and practical reasons, researchers are restricted in their ability to manipulate speech communities to measure the effects of particular variables. If language change is at issue, the investigator is faced with severe practical problems. As a proxy, data can be analysed by more than one contemporary generation of the same family or community, but this relies on the assumption that a speaker's patterns do not change over their lifetime. Studies like Harrington (2006), however, show that they do. Aside from these 
practical considerations, it is very hard to isolate the pressures and causal factors implicated in a particular change. No natural speech community is homogeneous: the linguistic input is not the same for all speakers, and this makes it very hard to compare the influence of different variables.

These problems have led to the use of computational simulations, which allow far greater manipulation of variables by the investigator. For example, Nettle and Dunbar (1997) simulate a population of 100 agents who exchange gifts that are of higher value to the recipient than to the giver, constituting a Prisoners' Dilemma game. Some of the agents are POLYGLOTS, who only give to organisms with a similar dialect to them, but will change their dialect to match that of another agent who gives them a gift. If allowed to cluster together, and given certain other parameters, 5 POLYGLOTS are able to invade a population of CHEATS (who accept gifts, but give none in return). Nettle (1999) describes a different set of simulations that model a population living on a spatial grid of $7 \times 7$ positions; each position on the grid is inhabited by a group of 20 individuals, which progress through 5 life stages before being replaced. By introducing imperfect learning, migration, social selection, and functional selection, he shows that social selection of linguistic variants "hugely increases both the amount of diversity which evolves and its stability" (54). Livingstone (2002) and Livingstone and Fyfe (1999) have challenged this view, with simulations that produce high levels of linguistic diversity without adaptive benefits. This is an important point: to say that social selection plays an important role in language diversity is not the same as saying it is a necessary condition for divergence; isolation and drift may well be sufficient conditions.

Livingstone (2002: 115) argues that simulation is valuable as it "allows the development of models which can incorporate different aspects of society [and] of language and language learning" in a way that "is not possible ... in real life". But such in silico approaches have their own downside. While they give the researcher far greater control, they offer, as Nettle (1999: 378) points out, only highly simplified models of the real world. They show us what computational agents do given certain assumptions, but we can not be sure that real human beings would do quite the same thing. With this in mind, there are perhaps two kinds of middle-ground approach that can complement both the methods described: the placing of simplified (usually robotic) agents in a real environment (e.g. Quinn 2001), and the placing of real human agents in a more or less artificial environment (e.g. Cornish 2006). The first approach has most advantage over fully computational simulations in cases where such behaviour as movement, vision, and the traversing of obstacles is involved. This paper describes a preliminary experiment of the second sort: it places human subjects in a simulated environment with an artificial language and an artificially imposed social structure.

\section{Overview}

In this experiment, participants played an online team-game that involved accumulating points by exchanging resources. Players negotiated for these resources in pairs on an instant-messenger style program using a made-up "alien language"; however, they did not know until after they had exchanged a particular item whether they had been negotiating with a team-mate or an opponent.

All participants began the game with exactly the same alien language (having been exposed to identical training data for the same amount of time). The experiment therefore simulated a situation in which a community had split into two parts, which were competing for resources ${ }^{5}$, yet still occupied a very close geographical area and were far from isolated one from the other: apart from two practice rounds (in which accumulated points were not counted), players interacted an equal amount of time with both teams.

\footnotetext{
5 The exchange of "resources" in the game should be interpreted as representing a broad range of activities in the real world, such as exchange of skills, or of knowledge, or indeed any form of cooperation towards a shared goal.
} 
The experiment aimed to investigate the following:

- Success at recognition.

- Effect on the alien language.

- Strategies employed.

In line with the hypothesis discussed above, there are several predictions to be made. First, players should be able to use linguistic cues to distinguish fellow team-members from members of the opposing team. It is crucial to note that the teams were not in any way distinguished linguistically at the start of the game (except inasmuch as they differed in their native languages or dialects, which they were not permitted to use). Any linguistic cues had to arise through innovation, whether intentional or accidental. Therefore, a second prediction is that the alien language should split into "dialects" or "accents" by which teams, or at least individuals, can be distinguished. Third, if the ability to recognise insider from outsider based on linguistic cues is adaptive, it can be predicted that the teams who do best at distinguishing between their team and the opposing team should achieve higher scores.

\title{
Participants
}

Twenty participants were selected from among students who responded to an advertisement on a student employment website. The experiment was described as a linguistics experiment that "involves playing a game with three other participants using an alien language". To avoid difficulties in understanding instructions, applicants were selected according to level of English: all but six were native speakers. Participants came from a wide variety of academic backgrounds, and none had studied more than a semester of linguistics. Ages ranged from 18 to 42. The experiment was run five times over a two-week period, and each run involved four participants, split into teams of two. Participants were assigned to runs according to availability and assigned to teams at random. ${ }^{7}$ They were not told who their team-mate was until after the game had finished. Everyone was paid $\ell^{8}$ for participating and a $£ 10$ bonus was shared between members of the winning team in each run.

\begin{abstract}
Alien language
Participants were not permitted to use English or any other real language during the experiment; numerals were also prohibited. Instead, they were given an "alien language" to learn, which consisted of nineteen randomly generated words, all of which had a CV syllable structure and were between two and three syllables long (e.g. ralu, tejizo). The training data consisted of a sheet of paper giving the nineteen made-up words with the following English glosses: hello, goodbye, please, thanks, yes, no/not, and, I, you, have, want/need, give, meat, grain, water, fruit, fish, much/a lot of, question word. Participants were asked to study this in silence for ten minutes, and were allowed to make notes on the sheet. They were not allowed to confer, and could not even see each other. For the last five minutes of the training session, they were also given access to a set of twelve online pictures showing cartoons of people using the language. There was a very small amount of variation in word order introduced here (the word glossed as "not", for example, might appear before the verb in one cartoon and after it in another). This was not, however, pointed out explicitly, and it is important to note that all participants in each game were given identical training data and shown identical pictures.

The language was regenerated for each run of the experiment and the length of words varied (in three of the runs, ten words had three syllables and nine, two; this was reversed in the

\footnotetext{
${ }^{6}$ The choice of the term "alien language" was based on Cornish (2006), who found it helped stimulate interest in participating in her project.

${ }^{7}$ There was one exception. In one case, two participants on the same run knew each other; they were put on separate teams.
} 
other two runs). Words that closely resembled English words or proper names were filtered out, along with homonyms or close homonyms.

\section{Procedure}

Participants were seated in adjacent closed cubicles, each containing a PC. Having read the instructions and spent ten minutes with the language training data, they played two practice rounds in which they communicated only with their team-mate (and were made aware of this fact); for the practice rounds, they were allowed to refer to their wordlists and had thirty seconds longer than in later rounds to converse with their partners.

After the practice rounds, participants were allowed one minute to relax and have a last look at their wordlists, which were then removed. They then played 14 rounds of the game (though were not told how many they would play). For half the rounds, players were paired with their team-mates; for the other half, with the opposing team. The order was randomised, but no player was ever paired with the same team for more than three rounds in a row. Participants were unaware of this and knew only that they would be paired with their team-mates for the first two rounds. In fact, this was the case for the first three rounds. ${ }^{8}$

The game proceeded as follows. Each player began with five resources (meat, grain, water, fruit and fish) in different quantities (which added up to 7 for each player). The distribution was the same in each game, and players were randomly assigned player numbers and teams (this was unrelated to the particular cubicle a participant chose to sit in). Throughout the entire game, each player could see their own resources (and only their own) displayed on the left of the screen. In each round, a player was paired with another player, who might or might not be a team-mate. Each round consisted of three timed stages: Negotiation, Exchange and Feedback. In the Negotiation Stage, players could (by typing messages) chat and negotiate for resources using the alien language. In the Exchange Stage, players could choose (by clicking on + and signs) to give away some or all of their resources to the person they had been chatting to, and were also asked to answer on a scale of 1 to 4 (where 1 means "Definitely not" and 4 means "definitely") the question, "Were you talking to someone on your own team?" In the Feedback Stage, they were reminded what they had given, told what they had received, and told whether or not they had been talking to a team-mate. Then a new round began, and so on until the last round had finished. At this point, players were told the overall scores for each team. The team with the best combined score was the winner.

Points were accumulated by exchanging resources. Crucially, any resource was worth twice as much to a receiver than to the giver. For example, if Player 1 gave 3 points of meat to Player 2, Player 2 would gain 6 points of meat (this is based on Nettle and Dunbar's (1997) simulation). As final scores were calculated by team, and not by player, it was therefore beneficial to give resources to one's team-mate and to receive resources from any other player. It was detrimental to one's success to give resources to the opposing team. To help stimulate negotiation, players were also encouraged to keep their team's resources balanced: as a penalty, the difference between a team's highest and lowest scoring resources was deducted from its total score at the end of the game.

In each round, the negotiation stage lasted 180 seconds, the exchange stage 30 seconds, and the feedback stage 20 seconds. In the practice rounds, the negotiation stage was 30 seconds longer. All text and decisions submitted by participants, along with time of submission, were recorded.

\footnotetext{
${ }^{8}$ This was intended to frustrate participants' expectations about the third round, where we might expect a bias towards assuming they would not be paired with their team-mates again. Of course, a similar bias was to be expected in the fourth round, but it was hoped that the results of the bias in the two rounds would cancel each other out.
} 


\section{Results}

According to post-experiment questionnaires, participants enjoyed taking part. Some guessed that the game was at least partly about identifying people based on language, although others thought it had been more concerned with how people learn language. Estimations of personal success ranged from "not very well" to "excellent". Two participants claimed to have been able to guess their team-mate's gender, but had no explanation of how, beyond "a gut feeling". One game was unfortunately cut short by a power failure in the middle of the ninth round. In Tables 1 to 3, the teams and players affected are marked with an asterisk. Results will be given both including and excluding data from this game.

Table 1: Correct judgements in Stage 2 for all players

\begin{tabular}{lllll}
\hline Player & $\begin{array}{l}\text { No. correct } \\
\text { judgements }\end{array}$ & $\begin{array}{l}\text { \% correct } \\
\text { judgements }\end{array}$ & $\begin{array}{l}\text { Correct } \\
\text { identification of } \\
\text { team-mate }\end{array}$ & $\begin{array}{l}\text { Correct } \\
\text { identification of } \\
\text { opponent }\end{array}$ \\
$1 \mathrm{a}$ & 8 & 66.67 & $1(20 \%)$ & $7(100 \%)$ \\
$1 \mathrm{~b}$ & 11 & 91.67 & $4(80 \%)$ & $7(100 \%)$ \\
$2 \mathrm{a}$ & 10 & 83.33 & $3(60 \%)$ & $7(100 \%)$ \\
$2 \mathrm{~b}$ & 9 & 75 & $2(40 \%)$ & $7(100 \%)$ \\
$3 \mathrm{a}^{*}$ & 5 & 83.33 & $1(50 \%)$ & $4(66.67 \%)$ \\
$3 \mathrm{~b}^{*}$ & 6 & 100 & $2(100 \%)$ & $4(66.67 \%)$ \\
$4 \mathrm{a}^{*}$ & 3 & 50 & $1(50 \%)$ & $2(33.33 \%)$ \\
$4 \mathrm{~b}^{*}$ & 5 & 83.33 & $2(100 \%)$ & $3(50 \%)$ \\
$5 \mathrm{a}$ & 8 & 66.67 & $3(60 \%)$ & $5(71.42 \%)$ \\
$5 \mathrm{~b}$ & 10 & 83.33 & $4(80 \%)$ & $6(85.71 \%)$ \\
$6 \mathrm{a}$ & 6 & 50 & $4(80 \%)$ & $2(28.57 \%)$ \\
$6 \mathrm{~b}$ & 10 & 83.33 & $3(60 \%)$ & $7(100 \%)$ \\
$7 \mathrm{a}$ & 9 & 75 & $3(60 \%)$ & $6(85.71 \%)$ \\
$7 \mathrm{~b}$ & 9 & 75 & $3(60 \%)$ & $6(85.71 \%)$ \\
$8 \mathrm{a}$ & 10 & 83.33 & $4(80 \%)$ & $6(85.71 \%)$ \\
$8 \mathrm{~b}$ & 8 & 66.67 & $2(40 \%)$ & $6(85.71 \%)$ \\
$9 \mathrm{a}$ & 10 & 83.33 & $3(60 \%)$ & $7(100 \%)$ \\
$9 \mathrm{~b}$ & 11 & 91.67 & $4(80 \%)$ & $7(100 \%)$ \\
$10 \mathrm{a}$ & 8 & 66.67 & $3(60 \%)$ & $5(71.43 \%)$ \\
$10 \mathrm{~b}$ & 10 & 83.33 & $3(60 \%)$ & $7(100 \%)$ \\
Total & 166 & 77 & 55 & 111 \\
\hline
\end{tabular}

Success at identification. Table 1 shows the number of correct judgements made by each player in the second (Exchange) stage of each round. This is based on responses to the question, "Were you talking to someone on your own team?" A reply of 1 or 2 is taken to mean "no" and 3 or 4 to mean "yes". Results from the first two rounds, in which players knew they were talking to their team-mates, are ignored for this purpose. Where participants neglected to respond to this question (as happened on seven occasions), their guess is inferred on the basis of trading behaviour, which was fairly transparent.

Overall, eighteen of the twenty players were correct in their judgement on at least $67 \%$ of rounds (the remaining two players were correct $50 \%$ of the time); or fifteen out of sixteen players if the second (asterisked) game is excluded. The mean for all players was $77 \%$ and the mode $83 \%$; these are the same if the second game is excluded. In total, 216 judgements were made over all the games, of which 166 were correct and 50 incorrect. In line with predictions, this is significantly better than chance: $\chi^{2}(1)=62.296, p<.001$ (excluding the second game: $\chi^{2}(1)=54.188, p<.001$, based on 147 correct judgements and 45 incorrect). 
As described above, players were paired with their team-mates in half of the rounds; however, in the first two rounds, they were made aware of this fact, and the judgements from these rounds are excluded from the analysis. Therefore 88 of the 216 judgements were from rounds in which players were paired with their team-mates. Of these, 55 were correct and 33 incorrect. This is significant at $p<.05\left(\chi^{2}(1)=5.5 ; \chi^{2}(1)=4.05\right.$ excluding the second game). For those rounds in which players were paired with members of the opposing team, 111 guesses were correct and 17 incorrect, which is highly significant: $\chi^{2}(1)=69.031, p<.001$ (excluding the second game: $\left.\chi^{2}(1)=81.385, p<.001\right)$. This would appear to suggest that participants were erring on the side of caution, and were biased towards assuming they were paired with their opponent. This may reflect an inherent conservatism, but is most probably the result of knowing who their partner was in the first two rounds (see above). This would have led to a bias in the third round and (when they turned out, against expectation, to be paired with their team-mates) again in the fourth round. In fact, only two guesses in the fourth round were incorrect, and of the 33 failures to identify team-mates, 14 occurred in the third round. Although these biases should cancel each other out to an extent, his is a problem that needs to be solved in future experiments.

Table 2: Definite judgements in Stage 2 for all players.

\begin{tabular}{llllll}
\hline Player & Game & $\begin{array}{l}\text { No. of } \\
\text { definite } \\
\text { guesses }\end{array}$ & No. correct & $\begin{array}{l}\text { Team score } \\
\text { before } \\
\text { penalty }\end{array}$ & $\begin{array}{l}\text { Team score } \\
\text { after penalty }\end{array}$ \\
1a & 1 & 6 & $4(67 \%)$ & 47 & 35 \\
$1 \mathrm{~b}$ & 1 & 5 & $5(100 \%)$ & 47 & 35 \\
$2 \mathrm{a}$ & 1 & 6 & $6(100 \%)$ & 46 & 33 \\
$2 \mathrm{~b}$ & 1 & 4 & $3(75 \%)$ & 46 & 33 \\
$3 \mathrm{a}^{*}$ & 2 & 2 & $1(50 \%)$ & 61 & 52 \\
$3 \mathrm{~b}^{*}$ & 2 & 3 & $3(100 \%)$ & 61 & 52 \\
$4 \mathrm{a}^{*}$ & 2 & 0 & 0 & 17 & 13 \\
$4 \mathrm{~b}^{*}$ & 2 & 4 & $4(100 \%)$ & 17 & 13 \\
$5 \mathrm{a}$ & 3 & 3 & $2(67 \%)$ & 55 & 41 \\
$5 \mathrm{~b}$ & 3 & 7 & $7(100 \%)$ & 55 & 41 \\
$6 \mathrm{a}$ & 3 & 5 & $2(40 \%)$ & 44 & 28 \\
$6 \mathrm{~b}$ & 3 & 0 & 0 & 44 & 28 \\
$7 \mathrm{a}$ & 4 & 5 & $5(100 \%)$ & 67 & 54 \\
$7 \mathrm{~b}$ & 4 & 2 & $2(100 \%)$ & 67 & 54 \\
$8 \mathrm{a}$ & 4 & 7 & $7(100 \%)$ & 82 & 52 \\
$8 \mathrm{~b}$ & 4 & 9 & $7(78 \%)$ & 82 & 52 \\
$9 \mathrm{a}$ & 5 & 9 & $8(89 \%)$ & 463 & 432 \\
$9 \mathrm{~b}$ & 5 & 10 & $9(90 \%)$ & 463 & 432 \\
$10 \mathrm{a}$ & 5 & 7 & $6(86 \%)$ & 35 & 28 \\
$10 \mathrm{~b}$ & 5 & 1 & $1(100 \%)$ & 35 & 28 \\
Total & & 96 & $82(85 \%)$ & & \\
\hline
\end{tabular}


Table 3: Combined correct judgements for teams and team scores.

\begin{tabular}{lllll}
\hline Team & Game & $\begin{array}{l}\text { Combined } \% \\
\text { correct } \\
\text { judgements }\end{array}$ & $\begin{array}{l}\text { Score before } \\
\text { penalty }\end{array}$ & $\begin{array}{l}\text { Score after } \\
\text { penalty }\end{array}$ \\
1 & 1 & 79.17 & 47 & 35 \\
2 & 1 & 79.17 & 46 & 33 \\
3 & 2 & 91.67 & 61 & 52 \\
4 & 2 & 66.67 & 17 & 13 \\
5 & 3 & 75 & 55 & 41 \\
6 & 3 & 66.67 & 44 & 28 \\
7 & 4 & 75 & 67 & 54 \\
8 & 4 & 75 & 82 & 52 \\
9 & 5 & 87.50 & 463 & 432 \\
10 & 5 & 75 & 35 & 28 \\
\hline
\end{tabular}

Table 2 shows the number of definite judgements per player (defined as a guess of 1 or 4), and the number of these that were correct. As might be expected, definite guesses were incorrect overall in a minority $(15 \%)$ of cases. In each game, the highest score before penalty was achieved by the team with the greater number of definite guesses. The winning team in each game was also the one with the higher combined percentage of correct judgements (see Table 3). The scores themselves, however, cannot be easily predicted from this recognition rate. The different strategies employed need to be taken into account.

Strategies. It is worth reiterating at this point the limits imposed by the alien language. Players were not allowed to use any real language during the game; the nineteen words they were given to use simply did not allow the explicit discussion of strategy between team-mates. In spite of this, strategies did emerge, and some teams and players used them to great effect.

The ideal player of this game would have a reliable way of recognising if they were paired with their team-mate and, if so, making it clear who they were. If they were paired with an opponent, they would pretend convincingly to be part of their opponent's team. They would also keep their resources balanced throughout in order to avoid a penalty at the end of the game. This last task is complicated by the fact that the penalty is based on the combined score; the ideal player must therefore either rely on their team-mate to do the same, or keep track of their teammate's resources sufficiently to compensate for imbalance. As team-mates' resources are hard to keep track of, and team-mates themselves not necessarily reliable, one or two teams hit upon the simpler tactic of just giving away as much as possible in rounds where they were knew they were paired together. This was the strategy employed by the most successful team, but it is an extremely risky one, and depends on there being a very reliable means of identifying one's own team-mate. Should that fail, a player might give away all their resources to the other team.

To identify themselves, players employed roughly five identifiable linguistic strategies:

- "Secret handshake": indicate affiliation through greeting.

- Mimicry: imitate the other person.

- “Accent spotting": identify idiosyncratic features of your team-mate's (and others') use of language.

- Salient innovation: use the language in a noticeably different way.

- Expressive innovation: modify the language to increase expressivity.

Such strategies can be, and were, combined. It is also not always possible to draw a clear distinction between them: any expressive innovation is likely to be salient, for instance. Strategies 
may also conflict: accent spotting becomes less effective if one's team-mate begins imitating the spotter. There are other difficulties in the case of strategies like mimicry: many participants said that they had used this strategy, but in a given case of two players' using the language in a similar way, it is not always clear if this should be interpreted as intentional mimicry (or on whose part). Owing to these difficulties, this section will not attempt a statistical comparison of the overall effect of strategy on score. Instead, it will focus on the behaviour of the most successful team: Team 9 (see Table 3).

The members of this team used all of the above-identified linguistic strategies to some extent, and used the reliability this gave them to exchange with each other as much as possible when they were paired together. Most noticeably, they hit upon a simple innovation that was both expressive and highly salient: they repeated the names of their resources to indicate quantity; for example, if "ralu" meant "water", "ralu ralu ralu" would mean "three points of water". As this became impractical as quantities increased, they repeated them in abbreviated form, using only the first letter of the resource name (so rrrrrrrrrr meant "ten points of water"). ${ }^{9}$ This not only allowed the efficient transmission of information; it also allowed reliable teammate recognition. Impressively, the team also managed to keep this innovation secret from the other team by using a "secret handshake". Instead of "sekudi", glossed as "hello", members of this team greeted with "jori noloku", glossed as "I have". In early rounds, this seems to have been used literally; the players simply dispensed with a greeting and got down to the business of listing resources. When it became clear that the other team still began each interaction with "sekudi", this stylistic difference became conventionalised. That "jori noloku" did indeed take on the status of a greeting is shown by the fact that it came to be repeated by both players before they actually said what resources they had. Members of Team 9 even began employing the further strategy of waiting at the start of each round for the other player to begin the interaction. If the other person greeted with "sekudi", they would reply with the same. In one case this backfired, as a member of Team 9 began with "sekudi" despite being paired with their teammate. Nothing was exchanged in this round. Perversely, this indicates how powerful and reliable the strategy had become. As can be seen from Table 3, Team 9 won by a margin of 403 points. It is also notable that they made the greatest number of definite guesses of any team (see Table 2).

Linguistic divergence. As described above, all players learnt an identical artificial language at the start of the game, and were not permitted to use any real language or numerals during the game. No participant broke this rule. There was, however, an enormous amount of innovation: although the language began with only 19 unique words, far more unique forms were used in the games themselves. In the third game, 120 unique forms were used (127, if forms used in the practice rounds are counted). Even in the game cut short by a power cut, 56 unique forms were used. Most of this seems to be the result of error, either of memory or in typing. For example, a word like "dikono" might be also appear as "dinoko" or "dikno". These can be considered as analogous to production errors in speech; many occur only once or twice in a given game. However, there is also evidence of propagation: whatever the source of linguistic innovation, it can be adopted by other language users and eventually become established in a particular dialect. It may happen, for example, that all players in a game use both "dikono" and "dinoko" at some point, but that one player uses the second more often, while the other players prefer the first. It may also happen that two teams may be distinguished by such a choice: one team may come to use "dinoko" more often, while the other continues to favour "dikono". In this case, we could speak of something like dialectal difference. A real-life analogy would be the use of /aks/ in place of /ask/ among certain communities of English speakers. Many if not most of these speakers also use /ask/ on certain occasions, but they are distinguished by the relative frequency

\footnotetext{
${ }^{9}$ Two other teams employed repetition in this way. One was Team 3, in the game that was cut short. Had the game continued, it seems likely they would have been the second most successful team overall. The other was Team 8 , who only hit upon the strategy late in the game.
} 
of the two variants in their language (by contrast, probably most English speakers utter /aks/ only as a rare production error). In fact, such patterns occurred in the case of several word-forms in the games. In itself, however, isolated cases like this do not tell us much. The question is whether the game set-up has led to a divergence of the alien language into two distinct forms; in other words, does the independent variable Team have an effect on the use of particular variants by players? Are players more like their team-mates than their opponents?

As a test, all the individual word-forms used in each game (not including the game that was cut short) were extracted and a frequency count derived for each player in the game. For example, Player 1 might have used "dikono" 25 times, "dinoko" 18 times and "dikno" 0 times, while the corresponding counts for Player 2 might have been 20, 23 and 7. All the word-forms were then divided into sets, 19 for each game (the second game was excluded). Thus "dikono", "dinoko" and "dikno" would all form one set, while "bivo", "bivvo" and "biivo" would form another. In a very few cases, where it was not at all certain which set a form should belong to, it was excluded from the analysis. Also excluded were any sets containing only one item. Where players repeated abbreviated forms of words, these were treated as one variant (so, for example, rrrrr and rrrrrrrr were counted as tokens of the same variant). The final tally was 54 sets. For each of these sets, the relative frequencies of variants were worked out for each player (so in a set of 4 items, the distribution for one player might be .25.25.5 and 0). The mean Euclidean distance was then measured between each pair of players. Each variant set was then scored: if all four players were closer to their team-mates than to their opponents, the set was given a score of 1; if members of only one team were closer to each other than to their opponents, the set was given a score of $0.5 .^{10}$ The result was a combined score of 19 for all sets. The raw frequency counts were shuffled randomly for each item and the data rescored; this was repeated 10,000 times. Scores of 13.5 or above occurred 0 times, which is highly significant $(p<.001)$.

\section{Discussion}

To summarise: the results from this game provide evidence that adult human beings are well able to correctly interpret patterns of linguistic variation and use the information gained to protect resources from freeriders, even after a very short time, little exposure to the language, and no means of explicitly discussing strategy. Moreover, such behaviour seems to be sufficient to cause an initially homogeneous language to diverge according to the structure of the community that speaks it.

Of course, this remains only a preliminary run of an experimental paradigm. This paper hopes above all to show that linguistic behaviour of the kind discussed here can be investigated in the laboratory, and that this complements fully computational and mathematical models and studies of actual speech communities. As a preliminary run, it is necessarily small-scale, and has certain shortcomings that result from this. First, to clearly establish that the behaviour in the game was the result of the particular pressure to distinguish between team-mate and opponent, a control is needed in which participants play the same game, but where there is no such pressure. Second, the number of players is small; participants have only to recognise one team-mate. It would be very interesting to see if even a slight increase in team size would have a significant impact. The issue of how players are paired in the first rounds also needs addressing. To give some grounding to the idea that players belong to a team, it seems reasonable, and a better approximation to reality, that they should be explicitly paired with their team-mates at the beginning of the game. As noted above, however, this creates an inevitable bias in the following round; pairing them unexpectedly with their team-mate in this round may go some way towards cancelling out this bias, but it is not entirely satisfactory. The best solution may be to make

\footnotetext{
${ }^{10}$ This is somewhat conservative scoring. Nothing was awarded where one member of a team was closer to their team-mate, but that team-mate was closer to an opponent.
} 
participants aware of their partners only in the practice rounds. Alternatively, a more radical change would be to have the teams emerge from the interactions.

It should be clear that the "alien language" used in the experiment was highly simplified in comparison with real human languages; moreover, it was written rather than spoken. It is therefore worth noting that much of the linguistic behaviour seen in the game has real-life analogues. Reduplication (the repetition of a word or part of a word) is used in various languages, usually to convey plurality or intensity (as in English "very very good"). Croft (2000: 75-6) also notes that communities with specific shared interests often save superfluous effort (and emphasise their group's exclusiveness) by abbreviating commonly used terms, as when wine connoisseurs refer to Cabernet Sauvignon as "Cab Sav" and Chardonnay as "Chard". The "secret handshake" strategy, moreover, recalls Cruttenden (1986: 169):

There are certain areas which are particularly susceptible to the idiosyncratic use of tones: Greetings, farewells and social formulas are one such area: the conventional way of intoning the equivalent of Good morning will vary from language to language; moreover, variation within one language in such areas will be sensitive to very subtle social conventions.

A final point worth mentioning is the issue of innovation, the "actuation riddle" posed by Weinreich et al. (1968): where does change come from? This experiment is not designed to answer this question. However, it is notable that there is practically no evidence of innovation for the sake of innovation to be found in the data. There is no reason to suppose, for example, that the vast majority of linguistic variation was not the result of error, even if such error-based forms were propagated. Team 9's use of "I have" as a greeting was innovative, but was merely an extension of its literal meaning: the secret-handshake use arose out of an accidental difference between the teams' style. The repetition and abbreviation of resource names was a salient change to the language, but it fulfilled an expressive function. At no point, in other words, is there evidence that players changed anything about the language purely for the sake of being different. The linguistic cues that allowed players to identify each other were parasitic on more functional linguistic behaviour. In this context, it is worth going back to Team 9's behaviour. As their resources increased in quantity, they seem to have paid less and less attention to keeping their resources balanced, and concentrated on simply exchanging as much as possible when they were paired together. Actually listing how many of each resource they had thus became redundant. In other words, not only did "jori noloku" become conventionalised as a greeting; any information exchanged between the players became only a means of identifying each other; the parasitic function of the language had become its primary function. This is particularly clear in the case of Team 9, but there is no reason to suppose it was not true of other teams. It would also appear to be the case for much human communication: the actual content of the message is often far less important than the way it's transmitted, or the fact it's transmitted at all.

\section{Acknowledgments}

Big thanks go to Karen Ludke, Remco Knooihuizen, Will Barras, Lauren Stewart, Hannah Cornish, Anna Martowicz, Cyprian Laskowski, Derek Murphy and Dorli Gschwandtner for happily taking part in very preliminary pilots of the experiment described here; further thanks go to the whole of the Language Evolution and Computation Research Unit at the University of Edinburgh for further piloting, feedback, advice and support, in particular to Dan Dediu, Alexandra Dima and Simon Kirby for their advice on data analysis. I am extremely grateful to April McMahon and Andrew Smith for their excellent supervisory input throughout this study, including reading over early drafts of this paper. I would also like to thank three anonymous reviewers for their insightful comments. Funding for this work comes from the Economics and Social Science Research Council, without whom it would never have happened. Very special 
thanks go to Justin Quillinan, who is a far better programmer than I'll ever be, and who did the difficult technical work in setting the game up. 


\section{References}

Axelrod R (1984/1990) The Evolution of Co-operation. London: Penguin Books

Boyd R, Gintis H, Bowles S, Richerson, PJ (2003) The evolution of altruistic punishment. Proceedings of the National Academy of Sciences 100(6): 3532-5.

Christiansen MH, Chater N (2007) Language as shaped by the brain.

http://www.isrl.uiuc.edu/ amag/langev/paper/christiansen07languageBrain.html

Cornish H (2006) Iterated learning with human subjects: an empirical framework for the emergence and cultural transmission of language. Unpublished masters thesis, University of Edinburgh, United Kingdom. http://www.ling.ed.ac.uk/lec/LEC/Research.html

Croft W (2000) Explaining Language Change: an Evolutionary Approach. Harlow: Longman

Cruttenden A (1986) Intonation. Cambridge: Cambridge University Press

De Roeck A, Johnson R, King M, Rosner M, Sampson G, Varile N (1982) A myth about centreembedding. Lingua 58: 327-40.

Deacon TW (1997/1998) The Symbolic Species: the Co-evolution of Language and the Brain.

New York/London: Norton

Dediu D, Ladd D R (2007) Linguistic tone is related to the population frequency of the adaptive haplogroups of two brain size genes, ASPM and Microcephalin. PNAS 104:10944-10949.

Dunbar RIM (1996) Grooming, Gossip and the Evolution of Language. London: Faber and Faber

Enquist M, Leimar O (1993) The evolution of cooperation in mobile organisms. Animal Behaviour 45: 747-57.

Evans B (2004) The role of social network in the acquisition of local dialect norms by Appalacian immigrants in Ypsilant, Michigan. Language Variation and Change 16(4): 153-67.

Garrod S, Doherty G (1994) Conversation, co-ordination and convention: an empirical investigation of how groups establish linguistic conventions. Cognition 53: 181-215

Hagen L K (2008) The bilingual brain: human evolution and second language acquisition. Evolutionary Psychology 6(1): 43-63

Kirby S (2000) Syntax without natural selection: how compositionality emerges from vocabulary in a population of learners. In: The Evolutionary Emergence of Language: Social Function and the Origins of Linguistic Form (Knight C, ed), 303-23. Cambridge: Cambridge University Press.

Kirby S and Hurford J (2002) The emergence of linguistic structure: an overview of the iterated learning model. In Simulating the Evolution of Language (Cangelosi A, Parisi D, eds), 121-48. London: Springer Verlag.

Labov W (1963) The social motivation of a sound change. Word 19: 273-309. 
Labov W (1990) The intersection of sex and social class in the course of linguistic change. Language Variation and Change 2: 205-254.

Labov W (2001) Principles of Linguistic Change. Volume 2: Social Factors. Malden MA/Oxford: Blackwell.

Lieberman E, Michel J, Jackson J, Tang T, Nowak MA (2007) Quantifying the evolutionary dynamics of language. Nature 449: 713-6.

Livingstone D (2002) The Evolution of dialect diversity. In Simulating the Evolution of Language (Cangelosi A, Parisi D, eds), 99-117. London: Springer Verlag.

Livingstone D, Fyfe C (1999) Modelling the evolution of linguistic diversity. In Advances in Artificial Life, Proceedings of the $5^{\text {th }}$ European Conference in Artificial Life, ECAL '99, Laussane, Switzerland (Floreano D, Nicoud J-D, Mondada F, eds). London: Springer Verlag: 704-8.

Nettle D, Dunbar RIM (1997) Social markers and the evolution of cooperative exchange. Current Anthropology 38(1): 93-9.

Nettle D (1999) Linguistic Diversity. Oxford: Oxford University Press.

Pagel M, Atkinson QD, Meade A (2007) Frequency of word-use predicts rates of lexical evolution throughout Indo-European history. Nature 449: 717-21.

Quinn M (2001) Evolving communication without dedicated communication channels in J. Kelemen \& P. Sosik (Eds.) Advances in Artificial Life: Sixth European Conference on Artificial Life, ECAL'99. London: Springer Verlag.

Steels L (2000) Language as a complex adaptive system. In Proceedings of PPSN VI (Schoenauer M, ed). Berlin: Springer Verlag.

Tooby J, Cosmides L (1990) On the universality and the uniqueness of the individual: the role of genetics and personality. Journal of Personality 27: 17-67.

Trudgill P (1972) Sex, covert prestige and linguistic change in the urban British English of Norwich. Language in Society 1: 179-96.

Weinreich U, Labov W, Herzog M (1968). Empirical foundations for a theory of language change. In Directions for Historical Linguistics: A Symposium. (Lehmann W P, ed.), 95-195. Austin: University of Texas Press.

Worden RP (1995) A speed limit for evolution. Journal of Theoretical Biology 176: 127-52.

Wray A, Grace GW (2007) The consequences of talking to strangers: evolutionary corollaries of influences on linguistic form. Lingua 117: 543-78. 
An Experimental Study of Social Selection and
Frequency of Interaction in Linguistic Diversity

Gareth Roberts

Language Evolution and Computation Research Unit School of Philosophy, Psychology and Language Sciences

University of Edinburgh

EH8 9AD

gareth@ling.ed.ac.uk 


\begin{abstract}
Computational simulations have provided evidence that the use of linguistic cues as group markers plays an important role in the development of linguistic diversity (Nettle \& Dunbar, 1997; Nettle, 1999). Other simulations, however, have contradicted these findings (Livingstone \& Fyfe, 1999; Livingstone, 2002). Similar disagreements exist in sociolinguistics (e.g. Labov, 1963, 2001; Trudgill, 2004, 2008a; Baxter et al., 2009). This paper describes an experimental study in which participants played an anonymous economic game using an instant-messenger-style program and an artificial 'alien language'. The competitiveness of the game and the frequency with which players interacted were manipulated. Given frequent enough interaction with team-mates, players were able to use linguistic cues to identify themselves. In the most competitive condition, this led to divergence in the language, which did not occur in other conditions. This suggests that both frequency of interaction and a pressure to use language to mark identity play a significant role in encouraging linguistic divergence over short periods, but that neither is sufficient on its own.
\end{abstract}

Keywords: Cooperation, Cultural evolution, Experimental, Historical linguistics, Language change, Sociolinguistics

The author is an ESRC funded PhD student in the Language Evolution and Computation Research Unit at the University of Edinburgh. His PhD is supervised by Prof. April M. S. McMahon and Dr Andrew D. M. Smith. 


\section{DRAFT-PLEASE DO NOT CITE WITHOUT PERMISSION OF THE AUTHOR}

This is an experimental study of language divergence. Participants played a team-game in which they used an instant-messenger-style program to negotiate anonymously for resources in an artificial 'alien language'. Frequency of interaction was manipulated, as was the competitive nature of the game. Given sufficiently frequent interaction and a pressure to use language as a source of social markers, the 'alien language' diverged significantly. Neither factor was sufficient on its own. The first section of the paper discusses the theoretical background; the second section discusses previous computational, sociolinguistic, and experimental studies of the role of identity marking and frequency of interaction in language change; and the remaining sections describe the experiment and discuss its implications.

\section{Why we need markers of identity}

In evolutionary biology, cooperative behaviour is behaviour that benefits another individual, and whose evolution is dependent on this benefit; several mechanisms have been identified by which this can become an evolutionary stable strategy (see West, Griffin, \& Gardner, 2007, for a useful summary). However, many of these mechanisms rely on individuals staying around long enough to face the consequences of cheating (Axelrod, 1984/1990, 169-91) and, among mobile social organisms like human beings, individuals can move relatively easily from community to community, exploiting each in turn (Enquist \& Leimar, 1993). A solution to the problem of distinguishing between members and non-members of one's community is to employ some kind of marker. The ideal marker must be relatively costly to obtain or imitate (cf. Irons, 2001; Sosis, 2003; Ruffle \& Sosis, 2007), and suitably salient, yet flexible enough to cope with changing group dynamics. Any trait that varies across human populations can serve as a marker of this sort, but among all possible candidates, linguistic variation seems an especially good source (cf. Nettle \& Dunbar, 1997, p. 98). An accent or dialect is a salient trait that is flexible both across generations and within a single lifetime (Harrington, 2006). Yet, despite this flexibility, learning new speech patterns takes a considerable time, and considerable exposure to the target; this appears, moreover, to be generally easier for children than for adults, for reasons that re- 
main controversial (see e.g. Penfield \& Roberts, 1959; Lenneberg, 1967; Newport, Bavelier, \& Neville, 2001; Abrahamsson \& Hyltenstam, 2008). There is no reason to suppose that linguistic traits are the only human traits to be used in this way, but linguistic markers are likely to be especially reliable, and there is evidence that even young children attach especial importance to them (Kinzler, Dupoux, \& Spelke, 2007; Kinzler, Shutts, DeJesus, \& Spelke, in press).

\section{Selection in language change}

If linguistic variation is exploited as a source of identity markers, this exploitation can be expected to influence the evolution of a language, since it introduces a selective pressure, usually called social selection (whereby variants are selected according to their association with something of social relevance to the speaker. See e.g. Nettle, 1999; Croft, 2000). We might expect this to lead to a high level of socially structured variation-whereby individuals converge linguistically with other members of their group, but groups diverge linguistically from each other-faster than would be expected by drift alone (Dunbar, 2003, p. 230). Indeed, a study of phylogenetic trees of language families by Atkinson et al. (2008) found evidence for 'punctuational bursts of evolution ... during the emergence of new and rival groups.'

This idea is familiar to sociolinguists, and Labov's (1963) description of a socially mediated sound change in Martha's Vineyard is a classic example (see also Blake \& Josey, 2003; Pope, Meyerhoff, \& Ladd, 2007). Such studies of real-world communities are complemented by computational models. Simulations by Nettle and Dunbar (1997) and Nettle (1999) appear to confirm that the existence of variability in language, coupled with a bias towards cooperating with those who speak in a similar way, leads to a population that cannot be invaded by 'cheats', and a high level of (geographically structured) linguistic diversity. Nevertheless, other simulations have cast doubt on the importance of social selection in explaining linguistic diversity; in studies by Livingstone and Fyfe (1999) and Livingstone (2002), a high level of geographically structured linguistic diversity emerges owing to variation in frequency of interaction, and no social selection.

This scepticism about the role of social selection is even echoed in the work of Labov, who argues that frequency of interaction is likely to be more important (Labov, 2001, p. 506). Similar views are expressed in Trudgill 
(2004) and Trudgill (2008a), and have been hotly debated (Bauer, 2008; Coupland, 2008; Holmes \& Kerswill, 2008; Mufwene, 2008; Schneider, 2008; Tuten, 2008; Trudgill, 2008b; Baxter et al., 2009). In psycholinguistics, Pickering and Garrod's mechanistic interactive alignment model of dialogue predicts that speakers will converge as a function of the amount they interact (Fay, Garrod, \& Carletta, 2000; Pickering \& Garrod, 2004). This does not seem to leave much space for more complex social factors.

Part of the difficulty in resolving this question is that the two factors of social selection and frequency interact considerably, and teasing them apart in the real world is difficult. Simulations in silico provide a solution to some of the practical difficulties but, as we have seen, give conflicting results, partly to be explained by the necessary simplicity of the agents that interact in them. A middle ground - a kind of simulation in vivo - is found in studies that put real human agents in a controlled artificial environment (e.g. Galantucci, 2005; Kirby, Cornish, \& Smith, 2008; Scott-Phillips, Kirby, \& Ritchie, in press). In a small-scale study of this sort described in Roberts (2008), participants were divided into two teams and played a game that involved typing messages anonymously on an instant messenger program using a simple artificial 'alien language' and exchanging resources with other players. The aim of the game was to share resources with team-mates, but to avoid giving them to opponents. The results suggested that the pressure to identify oneself to one's team-mate caused the alien language to diverge into team 'dialects'. However, the study did not investigate the role of frequency of interaction in bringing this about. The purpose of this paper is to present a larger-scale experimental study, based on Roberts (2008), that incorporates this factor.

\section{Description of experiment}

\subsection{Overview}

An experiment was carried out with 80 participants; it had a $2 \times 2$ (betweensubjects) design, so there were four conditions of 20 participants each. Five games were played in each condition, with four players in each game. A game consisted of a series of timed rounds in each of which every player was paired (anonymously) with one of the other players, their 'partner' for that round. The round involved negotiating anonymously, by typing messages in a simple 
artificial 'alien language' (the use of real languages was forbidden) on an instant-messenger-style program, to exchange resources with their partner. After this negotiation stage, players could choose to give resources away to their partner; any resource given was worth double to the receiver, which allowed teams to accumulate points.

In a typical round, players would greet each other, then state what resources they wanted and were prepared to give away, and ask what their partner wanted in return (e.g. 'I need lots of meat. Do you have meat? I give you fruit.'). Depending on this exchange, and the player's estimate of the benefits of doing so (see below), each player might then go on to give away a certain amount of their resources to their partner. After this they would be told the results of the exchange.

The first independent variable was competitiveness:

- Competitive: the four players were divided into two teams. The object of the game was to beat the opposing team by as great a margin as possible. There was a prize for the team in the condition that beat its opponents by the greatest margin.

- Cooperative: ${ }^{1}$ all four players were considered part of the same team. The object of the game was to get as high a score as possible. There was a prize for the team in the condition that achieved the highest score.

Every game of the cooperative condition was matched with a game in the competitive condition; for each pair, the words of the alien language and the order in which players were paired up was kept the same.

The second independent variable was frequency of interaction:

- Low-frequency: 15 rounds set up so that each player interacted an equal amount of time with every other player. In the competitive condition, this meant being paired only a third of the time ( 5 rounds) with their own team, and two thirds of the time (i.e. 10 rounds) with the opposing team.

- High-frequency: 16 rounds set up so that each player interacted half the time (i.e. 8 rounds) with one other player (always their team-mate in the competitive condition), and a quarter of the time (4 rounds) with each of the remaining two players. While there was thus a bias in 
terms of individual players, it meant that players interacted an equal amount of time with each team in the competitive condition.

Figure 1 is a diagram of the experimental design.

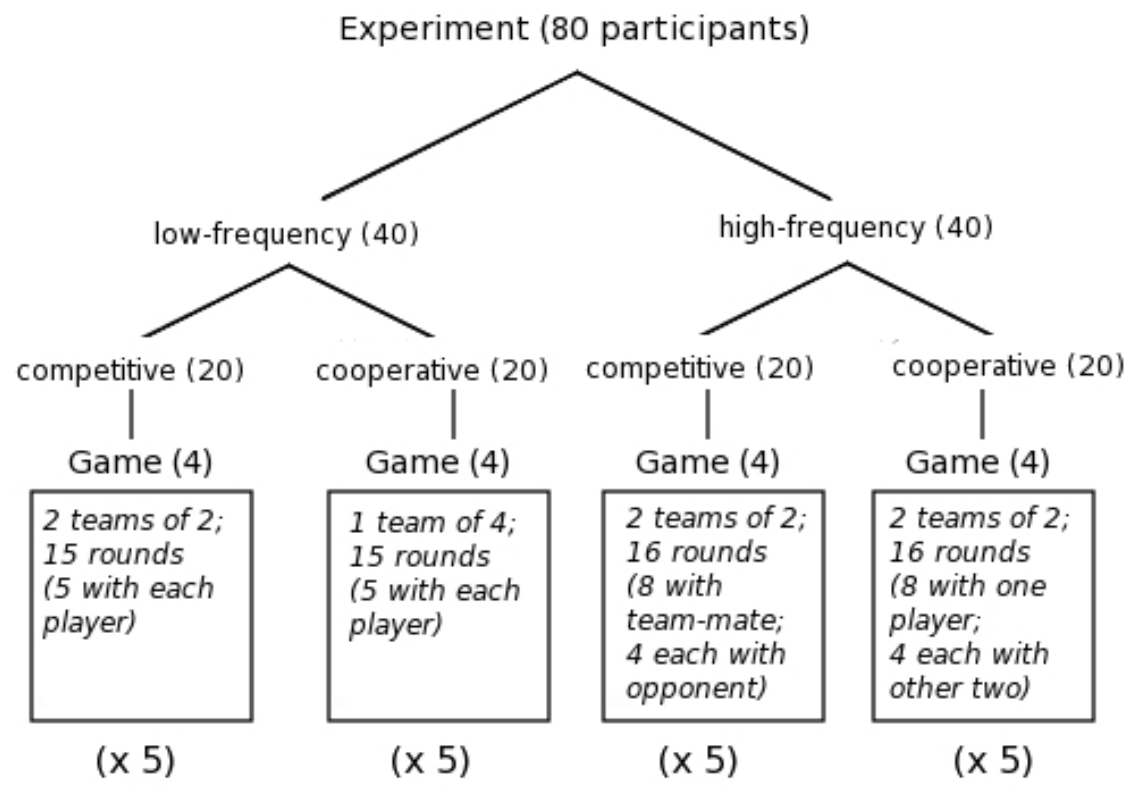

Figure 1: Diagram of experimental design (No. of participants given in brackets)

In the cooperative conditions, it will be noticed that the players' task was relatively straightforward: players had only to give as much as possible to their team-mates (i.e. all the other players in the game), while paying attention to what players asked for, so as to keep resources balanced. In the competitive conditions, the task was somewhat more complex. In addition to giving as much as possible to their team-mate (while keeping resources balanced), the ideal player would avoid ever giving resources away to their opponents, while always appearing to be the team-mate of whoever they were partnered with for a given round. This meant finding some way of identifying one's team-mate, and the only channel by which this could be achieved was the negotiation stage of the round. As will be seen in Section 3.5, however, 
the 'alien language' players had to communicate with severely limited their opportunity for explicit strategising; instead, players had to rely mainly on cues in the linguistic form of their messages, rather than the content.

With this in mind, two predictions were made for competitive condition:

1. That players would be able to identify their team-mates using only linguistic cues.

2. That the alien language, identical for all players at the beginning of the game, would diverge into dialects.

It was further predicted that these effects would be greater in the highfrequency condition, where team-mates were in greater contact.

\subsection{Participants}

Eighty participants were selected from among students who responded to an advertisement on a student employment website. The advertisement required that students should not apply if they suffer seriously from dyslexia or RSI, have studied linguistics, or have done an experiment for the same experimenter in the past. Participants were aged between 18 and 30, were a mixture of undergraduates and postgraduates from a variety of disciplines, and came from a variety of linguistic backgrounds. They were assigned to sessions according to their availability and assigned to teams at random. ${ }^{2}$ Every participant was paid $£ 8$ for participating. As a motivator, members of the most successful teams were awarded $£ 10$ each.

\subsection{Game structure}

Each player sat in a separate cubicle with a desktop computer. After they had all read through the instructions for the game, they were each presented with a sheet of paper with a list of twenty English words and glosses in an 'alien language' (see Section 3.5). They were given ten minutes to learn this, using any method that did not involve saying the words aloud. They were specifically told that they could write on the paper, and were given pens for this purpose. After five minutes had elapsed, they were directed on the screen to a set of cartoons with examples of the language being used. ${ }^{3}$ Every player learnt exactly the same alien words, and saw exactly the same cartoons. After training, participants played three practice rounds, in which they could 
refer to their word-lists. Players were paired with a different partner in each practice round. In the competitive condition, the first of these was their team-mate, and players were told as much. In the cooperative condition, they were told that each partner would be different. After the practice rounds were over, the word-lists were removed and the players played the full game, using the alien language from memory. Players were paired in the first three rounds of the full game as they had been in the practice rounds, and players in the competitive condition were again told that their first partner would be their team-mate. Players were told nothing else about who they would be paired with in each round, or how many rounds they would play. After the first three rounds, the order of pairings was random, though no two players were paired for more than two rounds in a row. A typical round was described in Section 3.1.

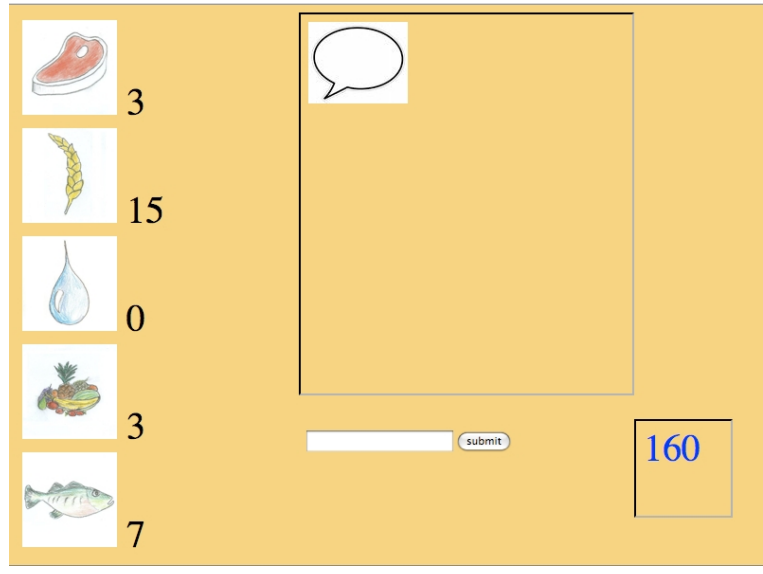

(a) CHAT Stage (in which players negotiate to exchange resources)

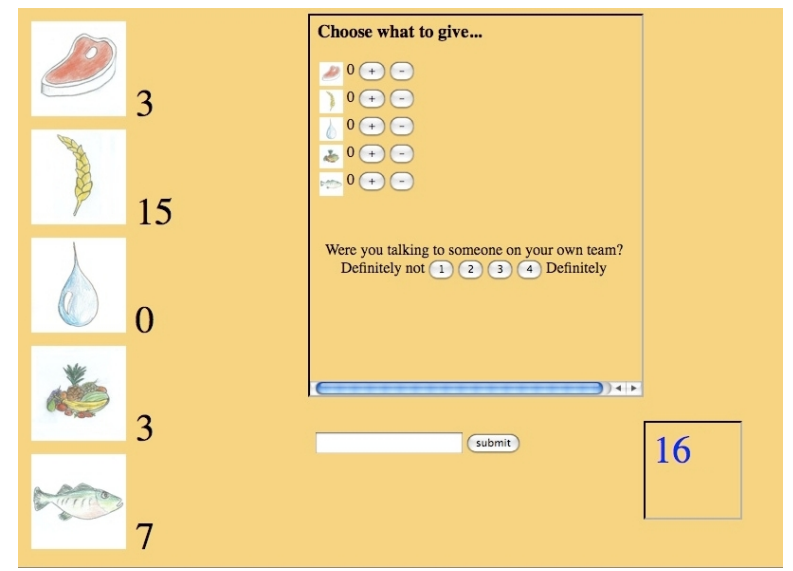

(b) EXCHANGE Stage (in which players exchange resources)

Figure 2: Examples of CHAT and EXCHANGE stages (Competitive condition)

For the duration of a round, each player was paired with one of the other players; except in the first round, they were given no information about who this was until the end of the round. Each round consisted of three stages. The first stage (CHAT; see Figure 2(a)) lasted for 160 seconds (120 in the practice rounds, which were otherwise identical to those in the full game), which pilots showed to constitute a considerable time pressure, considering that players could not use their native languages. Only during this stage could 
players communicate with each other, which they did by typing messages; no particular order of turns was imposed. The second stage (EXCHANGE; see Figure 2(b)) lasted 30 seconds. Only during this stage could players give away resources to their partner; there was no limit on gift size. In the competitive condition, they were also asked in this stage to judge if they had been talking to a member of their own team. In the third stage (FEEDBACK; see Figure 3(a)), which lasted 15 seconds, players were reminded how much of each resource they had given away, and told what they had received from the other player; in the competitive condition, they were also told whether or not the other player had been a member of their team. After that, a new round started, and so on until the end of the game, when the final score for both teams (or, in the cooperative game, the score for the whole group) was revealed (see Figure 3(b)).

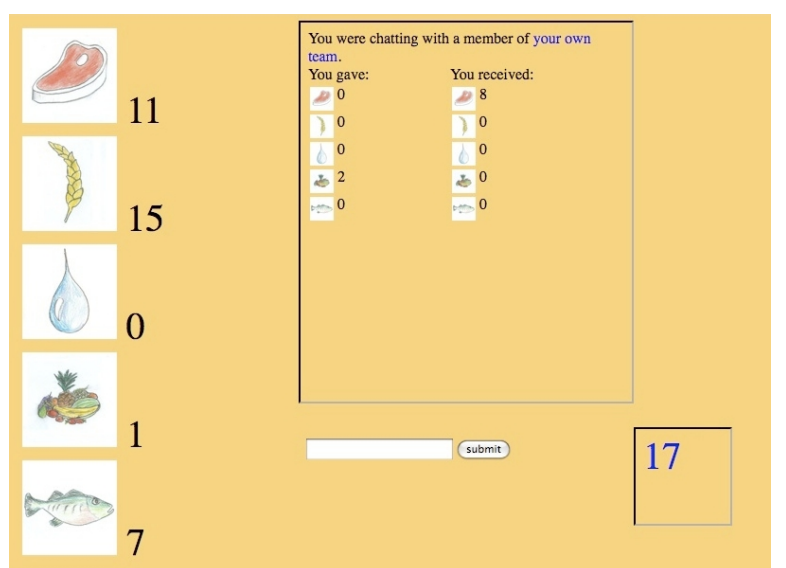

(a) FEEDBACK stage (in which players receive feedback on exchange and interlocutor's identity)

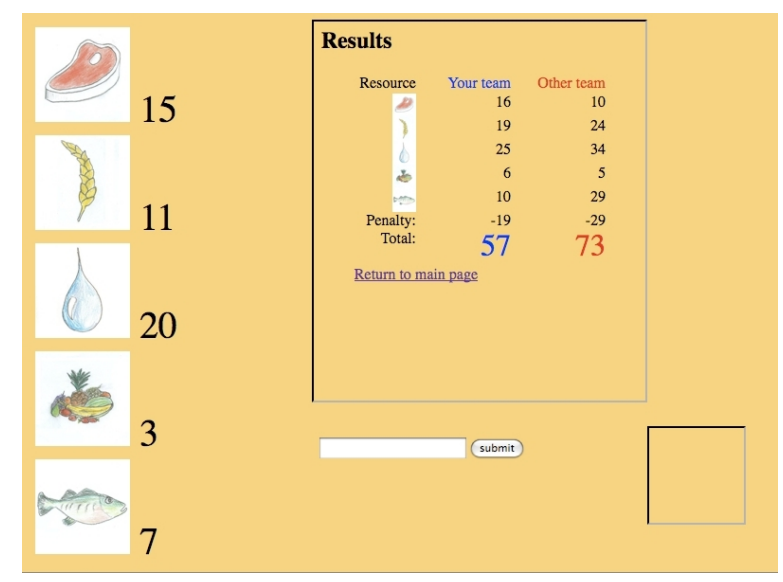

(b) Final screen (shows combined team scores at end of game)

Figure 3: Examples of FEEDBACK stage and final screen (Competitive condition)

\subsection{Resources and scoring}

Players began the game with 28 points each, distributed among the following resources: meat, grain, water, fruit and fish. Each player would begin with 0 points in one resource, 3 points in two, 7 points in one, and 15 points in another. No two players had identical distributions, but the 0 resource always 
corresponded to 15 in the player's team-mate's set. No resource was worth inherently more than another (10 points of meat, for example, was worth as much as 10 points of water). Throughout the game, players could see how much of each resource they had, but not how much their team, or any other player, had. The only way these quantities could change was by giving resources away to, or receiving them from, another player. Any resource given away was worth double to the receiver (cf. Nettle \& Dunbar, 1997); giving away resources to one's team-mate therefore allowed the team to accumulate points. The team's score for each resource was simply the sum of its members' scores. For the purposes of winning the game, it was the teams' scores, not the scores of their individual members, that mattered; there was thus a direct benefit to cooperating with members of one's own team. However, at the end of the game, teams were penalised for having unbalanced resources: the difference between the highest scoring resource and the lowest scoring resource was deducted from the total score (see Figure 3(b)). This was to discourage players from simply giving away all their resources, and to encourage them instead to use the alien language to negotiate a beneficial exchange. The only reason not to give away as much as possible to one's team-mateapart from uncertainty as to their identity in the competitive condition - was the possibility of dangerously unbalancing the team's resources. In the event, most players proved to be rather conservative, and the most successful teams were those who gave away large quantities of resources, but who established reliable markers to make this less risky (see Sections 4.4.1 and 4.4.2). Players did not see their team's score, or any score but their own, until the end of the game.

\subsection{Alien language}

Players were not allowed to use English, any other real language, or numerals in the experiment. Instead they were given an 'alien language' to learn, which consisted of twenty randomly generated words of either two or three CV syllables. This severely limited the potential for explicit strategising. The alien words were generated anew for each pair of games (that is, the same language was used for one game in the cooperative condition, and one in the competitive condition - although games in the high-frequency condition were not matched in this way with games in the low-frequency condition). The following is an example set, with English glosses: 


$\begin{array}{ll}\text { hello/goodbye }- \text { suto } & \text { need - ganita } \\ \text { please }- \text { gowene } & \text { give }- \text { ziga } \\ \text { thanks - wumovo } & \text { meat - nimu } \\ \text { yes - dejizi } & \text { grain - jemulo } \\ \text { no/not - falo } & \text { corn - kovuha } \\ \text { and - tuga } & \text { water - yane } \\ \text { I - takola } & \text { fruit - golo } \\ \text { you - bofa } & \text { fish }- \text { nesewi } \\ \text { have - hilapi } & \text { much/a lot of }- \text { firo } \\ \text { want - hiyi } & \text { question word }- \text { yukini }\end{array}$

So that players would remain focused on the game and the screen in front of them, and so that memory lapses (along with typing mistakes) would provide a source for 'natural' variation in the language, analogous to production errors and slips of the tongue in real language, players were not able to refer to a word-list during the game itself and had to use the alien language from memory. Early pilots had suggested that participants felt somewhat overwhelmed by the prospect of having to 'get the alien language right', so participants were also told not to 'worry at all about making mistakes in the language. The important thing is to make yourself understood without using English (or any other real language).' This was also intended to encourage players to communicate as naturally as possible, and not to treat the task as a memory test.

\section{Results}

As in Roberts (2008), there were two main dependent variables:

1. Players' success in distinguishing between team-mates and opponents.

2. The degree to which the alien language diverged into two 'dialects'.

Only in one condition, the high-frequency competitive condition, were players able to identify their team-mates; and only in this condition did the alien language diverge into dialects.

Tables 1 and 2 summarise the raw results for every team in the competitive conditions. Section 4.1 analyses players' success at recognising their 
team-mates; Section 4.2 analyses the level to which the alien language diverged; Section 4.3 discusses the relationship of these measures with players' success in the game; Section 4.4 discusses the strategies players used.

\begin{tabular}{|c|c|c|c|c|c|}
\hline & Score $^{a}$ & $\begin{array}{l}\text { Convergence } \\
\text { level }^{b}\end{array}$ & $\begin{array}{l}\% \text { Correct } \\
\text { recognitions }\end{array}$ & $\begin{array}{l}\text { Mean } \\
\text { gift }^{d}\end{array}$ & $\begin{array}{l}\text { Strategies } \\
\text { employed }^{e}\end{array}$ \\
\hline \multicolumn{6}{|c|}{ Game 1} \\
\hline Team a & $110(126)$ & 0.89 & 61.5 (12) & $2.3(4.1)$ & DR, II \\
\hline Team b & 102 (102) & 0.86 & $64.3(14)$ & $1.8(2.7)$ & DR, II \\
\hline \multicolumn{6}{|c|}{ Game 2} \\
\hline Team a & 114 (163) & 0.87 & 57.7 (12) & $2.4(4.0)$ & DR \\
\hline Team b & 114 (131) & 0.86 & $78.6(14)$ & 3.7 (6.2) & DR, M, II \\
\hline \multicolumn{6}{|c|}{ Game 3} \\
\hline Team a & $205(467)$ & 0.88 & $62.5(10)$ & 13.3 (23.2) & $\mathrm{DR}, \mathrm{M}$ \\
\hline Team b & $236(544)$ & 0.93 & $50(10)$ & $16.7(32.7)$ & DR, II \\
\hline \multicolumn{6}{|c|}{ Game 4} \\
\hline Team a & 129 (224) & 0.83 & $75(10)$ & $7.3(9.1)$ & DR \\
\hline Team b & $78(245)$ & 0.82 & $56(11)$ & $4.6(3.7)$ & DR \\
\hline \multicolumn{6}{|c|}{ Game 5} \\
\hline Team a & 131 (189) & 0.89 & 74.1 (13) & $5.2(6.1)$ & DR, II \\
\hline Team b & $110(207)$ & 0.86 & $65.4(12)$ & $4.2(4.3)$ & $\mathrm{DR}, \mathrm{M}$ \\
\hline
\end{tabular}

Table 1: Summary of data for competitive low-frequency condition. Notes: a) score before penalty given in brackets; b) 1 - mean distance between team-mates (higher figure means greater convergence); see Section 4.2. c) excludes first round and rounds where players made no response; number of responses made given in brackets. d) based on all rounds; mean gift to team-mates only given in brackets. e) see section 4.4.1. 


\begin{tabular}{|c|c|c|c|c|c|}
\hline & Score $^{a}$ & $\begin{array}{l}\text { Convergence } \\
\text { level }^{b}\end{array}$ & $\begin{array}{l}\% \text { Correct } \\
\text { recognitions }\end{array}$ & $\begin{array}{l}\text { Mean } \\
\text { gift }^{d}\end{array}$ & $\begin{array}{l}\text { Strategies } \\
\text { employed }^{e}\end{array}$ \\
\hline \multicolumn{6}{|l|}{ Game 6} \\
\hline Team a & $588(659)$ & 0.86 & 92.6 (12) & 20.8 (38.1) & $\mathrm{DR}, \mathrm{M}, \mathrm{II}, \mathrm{SH}$ \\
\hline Team b & 195 (251) & 0.92 & $72.4(14)$ & $4.2(6.8)$ & $\mathrm{DR}, \mathrm{M}$ \\
\hline \multicolumn{6}{|c|}{ Game 7} \\
\hline Team a & $59(89)$ & 0.92 & $60(15)$ & $2.1(2.5)$ & DR, II \\
\hline Team b & $141(149)$ & 0.89 & $68.97(14)$ & $1.8(3.1)$ & DR, II \\
\hline \multicolumn{6}{|c|}{ Game 8} \\
\hline Team a & 146 (162) & 0.87 & $65.5(14)$ & $3.9(6.1)$ & DR, M, II \\
\hline Team b & $122(161)$ & 0.86 & $83.3(15)$ & $2.7(4.4)$ & $\mathrm{DR}, \mathrm{M}, \mathrm{II}$ \\
\hline \multicolumn{6}{|c|}{ Game 9} \\
\hline Team a & $142(176)$ & 0.93 & 62.96 (12) & $4.3(6.1)$ & DR \\
\hline Team b & 1057 (1248) & 0.86 & $100(10)$ & $36.8(71.6)$ & DR, II, SH \\
\hline \multicolumn{6}{|c|}{ Game 10} \\
\hline Team a & $78(91)$ & 0.88 & $62.1(14)$ & $1.2(1.7)$ & DR \\
\hline Team b & $95(95)$ & 0.86 & $73.3(15)$ & $1.1(1.6)$ & DR, II \\
\hline
\end{tabular}

Table 2: Summary of data for competitive high-frequency condition. Notes: a) score before penalty given in brackets; b) 1 - mean distance between team-mates (higher figure means greater convergence); see Section 4.2. c) excludes first round and rounds where players made no response; number of responses made given in brackets. d) based on all rounds; mean gift to team-mates only given in brackets. e) see section 4.4.1. 


\subsection{Recognising team-mates}

In the second stage of each round in the competitive condition, players were asked: 'Were you talking to someone on your own team?' and answered on a scale of 1 to 4 , where 1 meant 'Definitely not' and 4 meant 'Definitely'. The third column of Tables 1 and 2 shows the percentage of correct recognitions for each team, where an answer of 1 or 2 is taken to mean 'no', and an answer of 3 or 4 is taken to mean 'yes'. ${ }^{4}$ As can be seen, players were more often right than wrong. All 258 judgements for the low-frequency condition were submitted to a binomial sign test. The result is significant $(S=91$ incorrect; $p<.001$ ), but less impressive than it seems. Players in this condition interacted more with the opposing team than their own. Had they guessed 1 or 2 every time, they would actually have done better $(S=80$ incorrect out of $280 ; p<.001)$.

It is possible, however, that these explicit judgements hide more impressive implicit judgements. To test whether players treated all assumed opponents the same, gifts were compared (as a proportion of the player's resources at the time of donation) for those rounds where they voted 'no': if players were talking to team-mates, did they give them more than they gave their opponents, despite explicitly guessing 'opponent' in both cases? In fact, a Wilcoxon $T$ test (where $N_{1}=32$ and $N_{2}=121$ ) showed that gifts to teammates were significantly larger in these rounds than gifts to opponents ( $T$ $=2504, p<.05$ ); furthermore, gifts to team-mates in these rounds did not differ significantly from gifts to team-mates where players guessed 'yes' ( $T$ $=913.5, N_{1}=32$ and $\left.N_{2}=61 ; p>.05\right)$. This suggests that, while players learnt that they were more likely to be paired with an opponent than a team-mate, and made their explicit judgements on this basis, they were still in many cases able to identify their team-mates, if only on a subconscious level.

In the high-frequency condition, players were paired equally often with opponents as with team-mates, and they did significantly better than chance at noticing which was the case $(S=75$ incorrect out of $285 ; p<.001)$. In this condition, there was no significant difference between gifts to opponents and team-mates on those rounds where players thought they were paired with opponents $\left(T=3620.5, N_{1}=49\right.$ and $\left.N_{2}=127 ; p>.05\right)$. 


\subsection{Linguistic divergence}

To measure the degree to which players converged and diverged linguistically, every individual word used in each game was extracted along with a frequency count for each player (i.e. how many times each player used a given form) and sorted into 'variable sets', according to which players could be compared. For example, one player might use only the form 'nimu' to refer to meat, while another player might use 'nimu' sometimes and (the innovative form) 'nimo' the rest of the time. These - along with any other forms used to mean 'meat' in a given game - would constitute a variable set. The innovative forms appear mainly to have risen accidentally, through typing mistakes or memory lapses, although several players claimed to have intentionally introduced small innovations (see Section 4.4.1). On the basis of these sets, pairs of players could be compared according to which variant they favoured. This is analogous to variation in real language, where speakers vary in the phonetic forms they use (e.g. the word 'meat' might be pronounced variously as [mit], [mi?], [mit], [mər?] etc.). Most variation was analogous to this kind of phonetic variation, and the introduction of entirely new forms - that had no obvious connection with the original form - was extremely rare: there were only two clear cases across all conditions (noti for $k e b i$ 'not'; and penah, and variations on it, for leba 'fish'). ${ }^{5}$

For each condition, all the forms used were assigned to variable sets as described and the raw frequency scores converted to proportions. The mean Euclidean distance between each pair of players was then calculated. Table 3 shows a simple example, taken from one game, of a small variable set consisting of two variants. The number on the left of each cell is the raw frequency and the number in brackets is the proportion. The form lale was given to participants in the word list at the start of the game, and glossed as 'you'. The variant lele most likely arose as an error, but was the only form Player 2 used throughout the game. Player 4, as it happened, was similarly consistent in her use of the other form. Table 4 shows the mean distance (in proportions) between every pair of players for this example set; the smallest distances are marked in bold.

The second column of Tables 1 and 2 shows the mean level of convergence for every team (calculated as 1 minus the mean distance between team-mates across variable sets; a score of 1 would indicate complete convergence). Only in Game 9 was there a significant difference $(p=0.035$, based on a Monte Carlo test) between the convergence level of the two teams. For the majority 
of games, therefore, both teams were converged to a similar level. It is important to note, however, that this is not a measure of between-team divergence: if all four players in a game used the language in exactly the same way, both teams would show the same level of convergence; on the other hand, two teams could show the same level of convergence, yet diverge significantly from each other. To test whether teams had diverged in any condition, the variable sets were scored: if, for a given variable set in the competitive condition, every player was closer to their team-mate than to either of their opponents (which is the case in the example set), the set was awarded a score of 1 (meaning that the teams had diverged with regard to this variable); if this was true of both members of only one team, the set was scored 0.5 ; otherwise, it was scored 0 (meaning no between-team divergence). The scores for all variable sets were summed in each condition. $P$ values were calculated using the Monte Carlo method (the data was shuffled and rescored 10,000 times). Precisely the same method was employed for the cooperative conditions, except that 'team-mate' here meant the player corresponding to a team-mate in the competitive condition, namely those who happened to be paired in the first round. In the high-frequency condition, this meant a player's team-mate was the player they were paired with most often. If social selection did not play a significant role in stimulating divergence, and frequency of interaction were sufficient on its own, we should thus not expect a significant difference between the competitive and cooperative high-frequency conditions, as the frequency of interaction was the same in the two conditions.

\begin{tabular}{lllll}
\hline & \multicolumn{2}{c}{ Team 1 } & \multicolumn{2}{c}{ Team 2 } \\
& Player 1 & Player 2 & Player 3 & Player 4 \\
\hline lale & $2(.2)$ & $0(0)$ & $12(.857)$ & $43(1)$ \\
lele & $8(.8)$ & $18(1)$ & $2(.143)$ & $0(0)$ \\
\hline
\end{tabular}

Table 3: Example variable set

The first two rows of Table 5 show the resulting $p$ values: there was significant divergence in only one condition. The competitive and cooperative conditions were also compared directly by performing a Monte Carlo test on the scores for each variable set. The results of this are shown in the third row of Table 5: there was a significant difference between conditions only in the high-frequency condition, suggesting that both a pressure to distinguish between team-mates and opponents and sufficiently frequent interaction with the former can cause divergence in the short term. 


\begin{tabular}{lllll}
\hline & \multicolumn{2}{c}{ Team 1 } & \multicolumn{2}{c}{ Team 2 } \\
& Player 1 & Player 2 & Player 3 & Player 4 \\
\hline Player 1 & 0 &. $\mathbf{2}$ & .657 & .5 \\
Player 2 &. $\mathbf{2}$ & 0 & .857 & .5 \\
Player 3 & .657 & .857 & 0 &. $\mathbf{1 4 3}$ \\
Player 4 & .5 & .5 & $\mathbf{. 1 4 3}$ & 0 \\
\hline
\end{tabular}

Table 4: Mean distances between players in example set

\begin{tabular}{lll}
\hline & high-frequency & low-frequency \\
\hline Competitive & $p=.0014^{* *}$ & $p=.566$ \\
Cooperative & $p=.937$ & $p=.275$ \\
Conditions compared & $p=.027^{*}$ & $p=.275$ \\
\hline
\end{tabular}

Table 5: Significance values for linguistic divergence per condition

\subsection{Comparison with score}

In four out of five competitive games in the high-frequency condition, the team that made the greatest number of correct judgements also won the game; and in the one game where this was not the case, the score before penalty was extremely close (161 to 162). This suggests, as expected, that there is a fitness advantage to being able to identify members of one's own community, which agrees with the finding in Roberts (2008).

The low-frequency condition was very different; the team that made the most correct guesses in this condition had the lower score before penalty in every case. This suggests (as is supported by players' own evaluations in feedback questionnaires) that players were not doing well at recognising their team-mates, at least on a conscious level (see Section 4.1). As noted above, players would be correct in their judgements two thirds of the time simply by guessing 1 or 2 every round.

In neither condition was there a significant correlation between level of convergence and score (low-frequency condition: $r(8)=.49, p>.05$; highfrequency condition: $r(8)=-.38, p>.05)$ and, as noted in Section 4.2, there was a significant difference between levels of within-team convergence in only one game - Game 9-where the most closely converged team was in fact the losing team. While this appears to contradict expectations, it is not as strange as it may appear. For team-mates to be able to identify each other, an especially high level of convergence is in fact unnecessary; one suitably 
salient variant or shared code is sufficient for recognition; and, as can be seen from Table 2, the winning team in Game 9 had a particularly powerful code: a 'secret handshake' (see Section 4.4.1). The overall levels of within-team convergence and between-team divergence are better treated as by-products of adaptive strategies rather than as strategies themselves. The next section discusses the strategies that players actually used.

\subsection{Strategy}

\subsubsection{Identification strategies}

The strategies available to players to identify, and be identified as, teammates can be summarised as follows: ${ }^{6}$

- Dialect recognition (DR): try to remember how different players use the language and identify them based on this. For example, a player in Game 8 noticed (and mentioned in feedback) that another player always spelt dobo as dovo.

- Intentional innovation (II): make changes to the language that are likely to be recognisable. For example, a player in Game 10 (team b) exaggerated the form of the greeting (suto) by repeating the final vowel (e.g. sutooo, sutooooooo etc.).

- Mimicry (M): try to sound as much like the person you're talking to as possible. For example, the team-mate of the 'sutooo' player in Game 10 also used the form 'sutooo' on two occasions.

- 'Secret handshake' (SH): say something at the beginning of the interaction that identifies you to your team-mate (and ideally not your opponent), and requires a specific response from them (see below for an example).

The strategies are referred to in abbreviated form in the last column of Tables 1 and 2. Inclusion in that column is based on players' own reporting in feedback questionnaires of the strategies they employed and, where this can be clearly seen, evidence in the data.

As the tables show, the rarest, but apparently most successful, strategy was 'secret handshake', which occurred in Games 6 and 9. The more sophisticated of the two handshakes evolved to have three parts: one player gave 
the word for 'hello': vobu; the other responded by repeating it twice: vobu vobu; and the first responded again with vobu vobu!!!. Both handshakes were reliable enough to allow members of this team to exchange large amounts of resources with little fear of being tricked by the opposing team, and both teams won their games. Considering the power of this strategy, it is notable that it was used so rarely. The strategy is costly, however: given the rather tight time constraints in the game, devising and attempting to establish a shared system of this kind is very difficult, particularly when there is uncertainty about the identity of one's interlocutor. There are no proper examples of it in the low-frequency condition, where the low frequency of interaction between team-mates made it especially difficult to establish. One player in Game 3 seems to have attempted to establish such a system by repeating the word for hello three times at the start of each round ('suve suve suve'), but her team-mate failed to catch on, and she gave up the endeavour. It is worth noting, however, that this player was also the least conservative player in the condition (she gave away a mean of 16.9 points per round; see Section 4.4.2), and the only player in the condition to give her opponents less than half what she gave her team-mate. Her team won the game and was the highest scoring team in the condition.

\subsubsection{Giving behaviour}

The fourth column of Tables 1 and 2 shows the mean amount of resources given away per round by each team, and the mean amount given to teammates (whether or not players guessed who they were paired with). The most successful teams were the most generous. In both conditions, however, most players were rather conservative in their gift-giving. Even in the first round, when players were told explicitly that they would be paired with their team-mates, only one player gave away everything, and only one other gave away more than half their resources. It is worth bearing in mind that there are very few real-life situations where giving away all your resources is a good idea. The importance of keeping resources balanced to avoid a penalty also encouraged caution.

Two teams stand out in both conditions as being the least conservative; these are the only teams with a mean gift of over 10 . There is a difference between the two conditions, however. In the low-frequency condition, the non-conservative players began giving away large amounts of resources in the last five rounds of the game - a behaviour that appears to have originated 
with one player in the eleventh round and spread to the others. Players also did not discriminate successfully between team-mates and opponents in their generosity. In the high-frequency condition, on the other hand, nonconservatives were more consistently generous: all gave away more than $20 \%$ of their resources on at least four rounds apart from the first (where all gave over 30\%). They were also more discriminating. It is not surprising that generosity only occurs as a consistent behaviour in those teams that developed 'secret handshakes'; not only does the secret handshake provide a more secure environment for generous giving, it is itself costly to establish, and thus a non-conservative behaviour.

\subsubsection{Relation between strategy and linguistic divergence}

The 'secret handshakes' discussed above have parallels in real-life linguistic behaviour. Cruttenden $(1986 / 1997,169)$ notes, for example, that greetings and other social formulas are 'particularly susceptible to the idiosyncratic use of tones'. Occurring early and almost obligatorily in social interactions, yet carrying little semantic load, they are a convenient focus for variation and a useful linguistic marker, and this can lead to divergence in greeting behaviour between groups of speakers (see also Manzini, Sadrieh, \& Vriend, 2009 for experimental evidence for the role of gestures like smiles, winks and handshakes in enhancing coordination by eliciting trust). While they are an impressive strategy, however, they are a special case. The main focus of this paper is on the large-scale, though subtler, divergence discussed in Section 4.2.

This divergence was mainly the result of the other linguistic strategies, but an important point should be noted: not all these strategies can necessarily be expected to lead to linguistic divergence or convergence (they did not in the other conditions, at any rate to a significant level) and, when they do, this is not necessarily the conscious aim of the individuals involved; divergence is better understood as a large-scale by-product, or 'epiphenomenon' (cf. Keller, 1994), of smaller-scale strategies on the part of individuals. An important strategy in this respect is mimicry, as this is the means by which variant forms are adopted. 'Intentional innovation' also plays a role, as, like accidental and unconscious innovation, it increases the number of new variants available for propagation between team-mates. Finally 'dialect recognition', the most commonly used strategy, encourages team-mates to converge, since convergence reduces the amount of information that needs to be mem- 
orised about one's team-mates' linguistic behaviour. This can be expected to become more and more important as group size increases, to the point where keeping track of variation on the individual level becomes impossible (cf. Dunbar, 1993).

\subsubsection{Summary}

The most successful strategy was a combination of generous giving and the intentional creation of complex salient markers. Both are potentially costly behaviours, but the latter helped create an environment in which the former was less risky. Two points should be borne in mind, however. First, most players engaged very little in either of these behaviours. The majority relied instead on recognising less salient patterns of variation, some innovation, mimicry, and a conservative exchange of resources. It is this behaviour, not the more sophisticated 'secret handshake' strategies that led to the divergence described in Section 4.2. Secondly, these strategies were very successful only where frequency of contact between fellow group members was sufficiently high. Where it was low, the establishment of complex shared codes seems to have been too difficult.

\section{Discussion}

There is disagreement in the literature regarding the relative roles of social selection and frequency of interaction in stimulating linguistic divergence. In this experiment, players were able to identify their team-mates reliably only when they communicated with them $50 \%$ of the time, but this frequency of interaction only led to linguistic divergence where there was pressure to use the language to mark identity. Neither of these factors was sufficient on its own. This is not to say that neither factor can ever be sufficient; it is at least plausible that much longer games would have seen divergence in other conditions. The results show that a combination of frequent interaction and pressure to distinguish in-group from out-group encourages a language to diverge over a very short period of time.

These results replicate the results of Roberts (2008), and support the findings of Nettle and Dunbar (1997) and Nettle (1999): a pressure for social selection increases the amount of divergence between groups in spite of inter-group contact; in addition, the level of isolation in the high-frequency 
condition was not sufficient to produce significant divergence in the absence of social selection. As Dunbar (2003, p. 230) accepts, however, there is no reason to reject drift as an explanation for language diversity over long time periods. In this respect, these results do not contradict the findings of Livingstone and Fyfe (1999) and Livingstone (2002).

The study has certain limitations. As each game requires four participants, the number of games is relatively small in each condition; the games were also relatively short. An obvious future direction would be to run largerscale and longer games. There was also little scope in the experiment for investigating the role of such factors as changing team dynamics, or greater communicative pressures to encourage meaning-based change (cf. Smith, 2007), which can all be expected to play a role in real-life language change. It would be interesting in the future to incorporate these factors. Finally, in contrast with the computational simulations described in Section 2, which all involved multiple generations of agents, this study involved only one generation of speakers - although the output of the previous generation is simulated by the word-lists and cartoons in the training rounds. It would be interesting to incorporate inter-generational change into the game by using the output of one set of players to create the word-lists for the next set (cf. Kirby et al., 2008). It is nonetheless striking that divergence can be observed in the laboratory in only one generation (and-given that the game consists of fewer than twenty rounds of a few minutes each-over a very short time period).

To think solely in terms of divergence would be misleading. This is how we normally perceive the phenomenon in question - we consider, for instance, that Proto-Romance diverged into the modern Romance languages - but convergence is of just as much importance. Indeed, divergence occurred in all conditions of the game: just as no two speakers of Italian can be expected to have identical idiolects, no two players in the game used the alien language in exactly the same way. The between-team divergence measured in the game, however, depends not only on the distance between teams, but also on the distance between team-mates. It is important to be clear about the direction of the relationship: within-team convergence does not necessarily mean between-team divergence, but the latter does imply the former. In other words, if team-mates sound like each other, this does not mean they don't sound like their opponents; to be able to say that one team does not sound like the other, on the other hand, we need to also be able to say that members of one team have something in common.

Divergence occurs in two ways: through innovation in a subset of speak- 
ers, and as a by-product of convergence (where not all speakers converge). Innovation can result from both error and intention; both occurred in the experiment. Convergence occurs when speakers imitate each other's patterns of usage. Where players claimed to have done this intentionally in the game, this has been referred to as mimicry; but there is a substantial literature showing that this behaviour consistently occurs on an unconscious level in dialogue (e.g. Giles, Coupland, \& Coupland, 1991; Shepard, Giles, \& Poire, 2001), while the interactive alignment model predicts convergence as a function of the amount of interaction (Pickering \& Garrod, 2004). Indeed, it is almost always reasonable to accommodate to one's interlocutor. Rationally, this is less necessary in the 'cooperative' condition of the game, where there's only one side to be on. However, the interactive alignment model predicts that speakers will accommodate in this condition too, and this is a reasonable expectation: alignment aids communicative success. Moreover, if alignment is advantageous in the way suggested, then it is likely operate on a 'rule-ofthumb' level (cf. Goodie, Ortmann, Davies, Bullock, \& Werner, 1999). Yet the results in Section 4.2 show a difference in levels of convergence between the competitive and cooperative games. The divergence in the competitive condition cannot therefore be explained purely on the basis of frequency of interaction. That is not to say that the results contradict the view that frequency of interaction alone may lead to linguistic divergence. They strongly suggest, however, that a pressure to select linguistic variants on the basis of their social significance plays an important role in speeding up this process.

\section{Acknowledgements}

The author would like to thank the ESRC for financial support, April McMahon and Andrew Smith for academic support, and Justin Quillinan for technical assistance. 


\section{Notes}

${ }^{1}$ It will be noted that even games in the 'cooperative' condition were competitive with respect to other groups of players in the same condition. The conditions are named with regard to within-game competition.

${ }^{2}$ There was one exception, where two participants in the same session turned out unexpectedly to know each other; they were put (unknown to them) on separate teams.

${ }^{3}$ They were not given this from the beginning of the training session, as early pilots had suggested that it distracted too much from the word-lists.

${ }^{4}$ The responses from the first round, where players know they are talking to their teammate, were ignored, as were rounds where players neglected to respond. It will also be recalled (see Section 3.3) that games in the high-frequency condition were one round longer than in the low-frequency condition.

${ }^{5}$ No distinction was made between different kinds of variant (e.g. on the basis of edit distance), and all the variation measured was on the individual-word level. Word order or punctuation, for example, was not taken into account; these sources of variation remain open to future investigation.

${ }^{6} \mathrm{~A}$ few players also claimed to have attempted to keep track of their team-mates' resources (based on what they offered and asked for) so as to identify them on this basis. Since they reported very little success at this, it will be ignored in the following analysis. It should also be noted that it is hard to identify cases of intentional mimicry in the data; this may therefore be underreported. 


\section{References}

Abrahamsson, N., \& Hyltenstam, K. (2008). The robustness of aptitude effects in near-native second language acquisition. Studies in Second Language Acquisition, 30, 481-509.

Atkinson, Q. D., Meade, A., Venditti, C., Greenhill, S. J., \& Pagel, M. (2008). Languages evolve in punctuational bursts. Science, 319(5863), 588.

Axelrod, R. (1984/1990). The evolution of co-operation. London: Penguin Books.

Bauer, L. (2008). A question of identity: A response to Trudgill. Language in Society, 37(2), 270-3.

Baxter, G. J., Blythe, R. A., Croft, W., \& McKane, A. J. (2009). Modeling language change: an evaluation of Trudgill's theory of the emergence of New Zealand English. Language Variation and Change, 21 (2), 257-96.

Blake, R., \& Josey, M. (2003). The /ay/ diphthong in a Martha's Vineyard community: What can we say 40 years after Labov? Language in Society, 32, 451-85.

Coupland, N. (2008). The delicate constitution of identity in face-to-face accommodation: A response to Trudgill. Language in Society, 37(2), $267-70$.

Croft, W. (2000). Explaining language change. Harlow: Pearson Education Ltd.

Cruttenden, A. (1986/1997). Intonation. Cambridge: Cambridge University Press.

Dunbar, R. I. M. (1993). Coevolution of neocortex size, group size and language in humans. Behavioral and Brain Sciences, 16(4), 681-735.

Dunbar, R. I. M. (2003). The origin and subsequent evolution of language. In M. H. Christiansen \& S. Kirby (Eds.), Language evolution (pp. 219-34). Oxford: Oxford University Press.

Enquist, M., \& Leimar, O. (1993). The evolution of cooperation in mobile organisms. Animal Behaviour, 45, 747-57.

Fay, N., Garrod, S., \& Carletta, J. (2000). Group discussion as ineractive dialogue or as serial monologue. Psychological Science, 11(6), 481-6.

Galantucci, B. (2005). An experimental study of the emergence of human communication systems. Cognitive Science, 29(5), 737-67.

Giles, H., Coupland, N., \& Coupland, J. (1991). Accommodation theory: Communication, context and consequence. In H. Giles, J. Coupland, \& N. Coupland (Eds.), Contexts of accommodation: Developments in 
applied sociolinguistics (pp. 1-68). Cambridge: Cambridge University Press.

Goodie, A. S., Ortmann, A., Davies, J. N., Bullock, S., \& Werner, G. M. (1999). Demons versus heuristics in artificial intelligence, behavioral ecology, and economics. In G. Gigerenzer, P. M. Todd, \& the ABC Research Group (Eds.), Simple heuristics that make us smart (pp. 32756). Oxford University Press.

Harrington, J. (2006). An acoustic analysis of "happy tensing" in the Queen's Christmas broadcasts. Journal of Phonetics, 34, 439-57.

Holmes, J., \& Kerswill, P. (2008). Contact is not enough: A response to Trudgill. Language in Society, 37(2), 273-7.

Irons, W. (2001). Religion as a hard-to-fake sign of commitment. In R. M. Nesse (Ed.), Evolution and the capacity for commitment (pp. 292-309). New York: Russell Sage Foundation.

Keller, R. (1994). On language change: The invisible hand in language. London: Routledge.

Kinzler, K. D., Dupoux, E., \& Spelke, E. S. (2007). The native language of social cognition. Proceedings of the National Academy of Sciences, $104(30), 12577-12580$.

Kinzler, K. D., Shutts, K., DeJesus, J., \& Spelke, E. S. (in press). Accent trumps race in guiding children's social preferences. Social Cognition.

Kirby, S., Cornish, H., \& Smith, K. (2008). Cumulative cultural evolution in the laboratory: An experimental approach to the origins of structure in human language. Proceedings of the National Academy of Sciences, $105(31), 10681-6$.

Labov, W. (1963). The social motivation of a sound change. Word, 19, 273-309.

Labov, W. (2001). Principles of linguistic change. volume 2: Social factors. Malden, MA: Blackwell.

Lenneberg, E. (1967). Biological foundations of language. New York: Wiley.

Livingstone, D. (2002). The evolution of dialect diversity. In A. Cangelosi \& D. Parisi (Eds.), Simulating the evolution of language (pp. 99-117). London: Springer-Verlag.

Livingstone, D., \& Fyfe, C. (1999). Modelling the evolution of dialect diversity. In D. Floreano, J.-D. Nicoud, \& F. Mondada (Eds.), Advances in Artificial Life, proceedings of the 5th European conference in Artificial Life, ECAL '99, Laussane, Switzerland (pp. 704-8). London: Springer-Verlag. 
Manzini, P., Sadrieh, A., \& Vriend, N. J. (2009). On smiles, winks and handshakes as coordination devices. Economic Journal, 119, 826-54.

Mufwene, S. S. (2008). Colonization, population contacts, and the emergence of new language varities: A response to Peter Trudgill. Language in Society, 37(2), 254-8.

Nettle, D. (1999). Linguistic diversity. Oxford: Oxford University Press.

Nettle, D., \& Dunbar, R. I. M. (1997). Social markers and the evolution of cooperative exchange. Current Anthropology, 38(1), 93-9.

Newport, E., Bavelier, D., \& Neville, H. (2001). Critical thinking about critical periods. In D. Dupoux (Ed.), Language, brain, and cognitive development: Essays in honor of Jacques Mehler. Cambridge, MA: MIT Press.

Penfield, W., \& Roberts, L. (1959). Speech and brain mechanisms. Princeton, NJ: Princeton University Press.

Pickering, M., \& Garrod, S. (2004). Towards a mechanistic psychology of dialogue. Behavioral and Brain Sciences, 27, 169-226.

Pope, J., Meyerhoff, M., \& Ladd, D. R. (2007). Forty years of language change on Martha's Vineyard. Language, 83(3), 615-27.

Roberts, G. (2008). Language and the freerider problem: an experimental paradigm. Biological Theory, 3(2), 174-83.

Ruffle, B. J., \& Sosis, R. (2007). Does it pay to pray? Costly ritual and cooperation. B.E. Journal of Economic Analysis and Policy, 7, 1-35.

Schneider, E. W. (2008). Accommodation versus identity? A response to Trudgill. Language in Society, 37(2), 262-67.

Scott-Phillips, T., Kirby, S., \& Ritchie, G. R. S. (in press). Signalling signalhood and the emergence of communication. Cognition.

Shepard, C. A., Giles, H., \& Poire, B. A. L. (2001). Communication accommodation theory. In W. P. Robinson \& H. Giles (Eds.), The new handbook of language and social psychology (pp. 33-56). New York: Wiley.

Smith, A. D. M. (2007). Language change and the inference of meaning. In C. Lyon, C. Nehaniv, \& A. Cangelosi (Eds.), Emergence of communication and language (pp. 323-7). London: Springer-Verlag.

Sosis, R. (2003). Why aren't we all Hutterites: Costly signaling theory and religious behavior. Human Nature, 14 (2), 91-127.

Trudgill, P. (2004). New-dialect formation: The inevitability of colonial Englishes. Edinburgh: Edinburgh University Press.

Trudgill, P. (2008a). Colonial dialect contact in the history of European lan- 
guages: On the irrelevance of identity to new-dialect formation. Language in Society, 37(2), 241-54.

Trudgill, P. (2008b). On the role of children, and the mechanical view: A rejoinder. Language in Society, 37(2), 277-80.

Tuten, D. N. (2008). Identity formation and accommodation: Sequential and simultaneous relations. Language in Society, 37(2), 259-62.

West, S. A., Griffin, A. S., \& Gardner, A. (2007). Social semantics: Altruism, cooperation, mutualism, strong reciprocity and group selection. Journal of Evolutionary Biology, 20(2), 415-32. 


\section{References}

Abrahamsson, N. and Hyltenstam, K. (2008), 'The robustness of aptitude effects in near-native second language acquisition', Studies in Second Language Acquisition 30, 481-509.

Alexander, R. D. (1987), The Biology of Moral Systems, Aldine de Gruyter, New York.

Andersen, H. (1989), Understanding linguistic innovations, in L. E. Breivik and E. H. Jahr, eds, 'Language Change: Contributions to the Study of its Causes', Mouton de Gruyter, Berlin, pp. 5-28.

Andersen, H. (2006), Synchrony, diachrony and evolution, in O. N. Thomsen, ed., 'Competing Models of Linguistic Change: Evolution and Beyond', Current Issues in Linguistic Theory, John Benjamins, Amsterdam, pp. 59-90.

Arbib, M. A. (2005), 'From monkey-like action recognition to human language: An evolutionary framework for neurolinguistics', Behavioral and Brain Sciences 28(2), 105-24.

Atkinson, Q. D., Meade, A., Venditti, C., Greenhill, S. J. and Pagel, M. (2008), 'Languages evolve in punctuational bursts', Science 319(5863), 588.

Axelrod, R. (1984/1990), The Evolution of Co-operation, Penguin Books, London. Axelrod, R. and Hamilton, W. D. (1984), The evolution of cooperation in biological systems, in R. Axelrod, ed., 'The Evolution of Co-operation', Penguin Books, London, chapter 5, pp. 88-105.

Barash, D. P. (1982), Sociobiology and Behavior, 2nd edn, Elseview, New York.

Bargh, J. A. (1989), Conditional automaticity: Varieties of automatic influence in social perception and cognition, in J. S. Uleman and J. A. Bargh, eds, 'Unintended Thoughts', Guilford Press, London, pp. 3-51. 
Barkow, J. H. (2006), 'Vertical/compatible integration versus analogizing with biology.', Behavioral and Brain Sciences 29(4), 348-9.

Bateson, P. (1983a), Optimal outbreeding, in P. Bateson, ed., 'Mate Choice', Cambridge University Press, Cambridge, pp. 257-78.

Bateson, P., ed. (1983b), Mate Choice, Cambridge University Press, Cambridge.

Bauer, L. (2008), 'A question of identity: A response to Trudgill', Language in Society 37(2), 270-3.

Baxter, G. J., Blythe, R. A., Croft, W. and McKane, A. J. (2009), 'Modeling language change: an evaluation of Trudgill's theory of the emergence of New Zealand English', Language Variation and Change 21(2), 257-96.

Beecher, M. D. (1982), 'Signature systems and kin recognition', American Zoologist 22, 477-90.

Bergen, B. K. (2004), 'The psychological reality of phonaesthemes', Language 80(2), 290-311.

Blackmore, S. J. (2000), The Meme Machine, Oxford University Press, Oxford.

Blackmore, S. J. (2003), 'Consciousness in meme machines', Journal of Consciousness Studies 10(4-5), 19-30.

Blake, R. and Josey, M. (2003), 'The /ay/ diphthong in a Martha's Vineyard community: What can we say 40 years after Labov?', Language in Society 32, 451-85.

Bock, J. K. (1986), 'Syntactic persistence in language production', Cognitive Psychology 18, 355-87.

Bod, R. and Cochran, D. (2007), Introduction to exemplar-based models of language acquisition and use, in R. Bod and D. Cochran, eds, 'Proceedings of the ESSLLI Workshop "Exemplar-Based Models of Language Acquisition and Use", ESSLLI 2007.

Bod, R., Hay, J. and Jannedy, S., eds (2003), Probabilistic Linguistics, MIT Press, Cambridge, MA.

Boehm, C. (1997), 'Impact of the human egalitarian syndrome on Darwinian selection mechanics', The American Naturalist 150, S100-S121.

Bourhis, R. Y. and Giles, H. (1977), The language of intergroup distinctiveness, in H. Giles, ed., 'Language, Ethnicity and Intergroup Relations', Academic Press, London/New York, pp. 119-34. 
Bourhis, R. Y., Giles, H., Leyens, J. P. and Tajfel, H. (1979), Psycholinguistic distinctiveness: Language divergence in Belgium, in H. Giles and R. S. Clair, eds, 'Language and Social Psychology', Blackwell, Oxford, pp. 158-85.

Boyd, R., Gintis, H., Bowles, S. and Richerson, P. J. (2003), 'The evolution of altruistic punishment', Proceedings of the National Academy of Sciences 100(6), 35325.

Boyd, R. and Richerson, P. J. (1985), Culture and the Evolutionary Process, The University of Chicago Press, Chicago/London.

Boyd, R. and Richerson, P. J. (1987), 'The evolution of ethnic markers', Cultural Anthropology 2, 65-79.

Boyd, R. and Richerson, P. J. (2000), Memes: Universal acid or a better mousetrap, in R. Aunger, ed., 'Darwinizing Culture: The Status of Memetics as a Science', Oxford University Press, Oxford, pp. 143-62.

Branigan, H. P., Pickering, M. J., McLean, J. F. and Cleland, A. A. (2007), 'Syntactic alignment and participant role in dialogue', Cognition 104, 163-97.

Branigan, H. P., Pickering, M. J., Stewart, A. J. and McLean, J. F. (2000), 'Syntactic priming in spoken production: Linguistic and temporal interference', Memory and Cognition 28(8), 1297-302.

Brighton, H., Kirby, S. and Smith, K. (2005), Cultural selection for learnability: Three principles underlying the view that language adapts to be learnable, in M. Tallerman, ed., 'Language Origins: Perspectives on Evolution', Oxford University Press, chapter 13.

Brighton, H., Smith, K. and Kirby, S. (2005), 'Language as an evolutionary system', Physica of Life Reviews 2, 177-226.

Brown, E. J. (2010), Coordinated multi-modal expression and embodied meaning in the emergence of symbolic communication, in A. D. M. Smith, M. Schouwstra, B. de Boer and K. Smith, eds, 'The Evolution of Language: Proceedings of the 8th International Conference (EVOLANG8)', World Scientific, Singapore, pp. 375-6. Bulwer, J. (1644), 'Chirologia: On the natural language of the hand', London. Bybee, J. (2006), 'From usage to grammar: The mind's response to repetition', Language 82(4), 710-33. 
Bybee, J. L. (2007), Frequency of Use and the Organization of Language, Oxford University Press, Oxford.

Byrne, R. and Whiten, A., eds (1988), Machiavellian Intelligence, Oxford University Press, Oxford.

Cangelosi, A. and Parisi, D., eds (2002), Simulating the Evolution of Language, Springer-Verlag, London.

Chambers, J. K. and Trudgill, P. (1998), Dialectology, second edn, Cambridge University Press, Cambridge.

Chater, N., Reali, F. and Christiansen, M. H. (2009), 'Restrictions on biological adaptation in language evolution', Proceedings of the National Academy of Sciences 106, 1015-20.

Chomsky, N. (1986), Knowledge of Language: Its Nature, Origin and Use, Praeger, Westport.

Christiansen, M. H. and Kirby, S. (2003), Language evolution: The hardest problem in science?, in M. H. Christiansen and S. Kirby, eds, 'Language Evolution', Oxford University Press, Oxford, pp. 1-15.

Clark, A. and Chalmers, D. J. (1998), 'The extended mind', Analysis 58(1), 7-19.

Clutton-Brook, T. (2009), 'Cooperation between non-kin in animal societies', $N a$ ture 462(5), 51-7.

Cohen, E. and Cooper, R. L. (1986), 'Language and tourism', Annals of Tourism Research 13, 535-63.

Condillac, E. B. (1746), Essai sur l'origine des conaissances humaines, ouvrage ou l'on réduit à un seul principe tout ce qui concerne l'entendement humain, in 'CEuvres Philosophiques de Condillac', Georges LeRoy, Paris.

Corballis, M. C. (2002), From Hand to Mouth: The Origins of Language, Princeton University Press, New Jersey.

Coupland, N. (2008), 'The delicate constitution of identity in face-to-face accommodation: A response to Trudgill', Language in Society 37(2), 267-70.

Coupland, N. and Jaworski, A. (1997), 'Relevance, accommodation, and conversation: Modeling the social dimension of communication', Multilingua 16, 235-58.

Crockford, C., Herbinger, I., Vigilant, L. and Boesch, C. (2004), 'Wild chimpanzees produce group-specific calles: a case for vocal learning', Ethology 110, 221-43. 
Croft, W. (2000), Explaining Language Change, Pearson Education Ltd, Harlow.

Dale, R. and Lupyan, G. (2010), Squiggle: Large-scale social emergence of simple symbols, in A. D. M. Smith, M. Schouwstra, B. de Boer and K. Smith, eds, 'The Evolution of Language: Proceedings of the 8th International Conference (EVOLANG8)', World Scientific, Singapore, pp. 391-2.

Daly, M. and Wilson, M. I. (1982), 'Whom are newborn babies said to resemble?', Ethology and Sociobiology 3, 69-78.

Darwin, C. (1879/2004), The Descent of Man, 2nd edn, Penguin Books, London.

Dawkins, R. (1982/1999), The Extended Phenotype, Oxford University Press, Oxford.

Dawkins, R. (1989), The Selfish Gene, 2nd edn, Oxford University Press, London.

de Bot, K. (2008), 'Review article: The imaging of what is in the multilingual mind?', Second Language Research 24(1), 111-33.

De Houwer, A. (2009), Bilingual First Language Acquisition, Multilingual Matters, Bristol.

de Oliveira, P. M. C., Stauffer, D., Wichmann, S. and de Oliveira, S. M. (2008), 'A computer simulation of language families', Journal of Linguistics 44, 659-75.

de Saussure, F. (1916/1995), Cours de Linguistique Générale, Payot, Paris/Lausanne.

Deacon, T. W. (1997), The Symbolic Species: The Co-Evolution of Language and the Brain, Norton, New York.

Deacon, T. W. (2003), Multilevel selection in a complex adaptive system: The problem of language origins, in B. H. Weber and D. J. Depew, eds, 'Evolution and Learning', MIT Press, Cambridge, MA, pp. 81-106.

Dennett, D. C. (1990), 'Memes and the exploitation of imagination', The Journal of Aesthetics and Art Criticism 48(2), 127-35.

Dennett, D. C. (1993), Consciousness Explained, Penguin Books, London.

Dennett, D. C. (1996), Darwin's Dangerous Idea: Evolution and the Meanings of Life, Penguin Books, London.

Diggle, S. P., Gardner, A., West, S. A. and Griffin, A. S. (2007), 'Evolutionary theory of bacterial quorum sensing: When is a signal not a signal?', Philosophical Transactions of the Royal Society B 362, 1241-9. 
Dudley, S. A. and File, A. L. (2007), 'Kin recognition in an annual plant', Biology Letters 3, 435-8.

Dudley, S. A. and File, A. L. (2008), 'Yes, kin recognition in plants!', Biology Letters 4, 69-70.

Dufwenberg, M. and Muren, A. (2006), 'Generosity, anonymity, gender', Journal of Economic Behavior \& Organization 61(1), 42-9.

Dunbar, R. I. M. (1993), 'Coevolution of neocortex size, group size and language in humans', Behavioral and Brain Sciences 16(4), 681-735.

Dunbar, R. I. M. (1996), Grooming, Gossip and the Evolution of Language, Faber and Faber, London.

Dunbar, R. I. M. (1998), 'The social brain hypothesis', Evolutionary Anthropology 6, 178-90.

Dunbar, R. I. M. (2003), The origin and subsequent evolution of language, in M. H. Christiansen and S. Kirby, eds, 'Language Evolution', Oxford University Press, Oxford, pp. 219-34.

Dunbar, R. I. M. and Shultz, S. (2007), 'Evolution in the social brain', Social Cognition 317, 1344-7.

Edgington, E. S. (1995), Randomization Tests, 3rd edn, Marcel Dekker, New York.

Emmorey, K. (2005), 'Sign languages as problematic for a gestural origins theory of language evolution', Behavioral and Brain Sciences 28, 130-1.

Enquist, M. and Leimar, O. (1993), 'The evolution of cooperation in mobile organisms', Animal Behaviour 45, 747-57.

Evans, N. and Levinson, S. (2009), 'The myth of language universals: Language diversity and its importance for cognitive science', Behavioral and Brain Sciences 32(5), 429-92.

Everett, D. (in preparation), Cognitive Fire: Language as a Cultural Tool, Profile, London.

Fehr, E. and Fischbacher, U. (2004), 'Third-party punishment and social norms', Evolution and Social Behavior 25, 63-87.

Fehr, E. and Rockenbach, B. (2003), 'Detrimental effects of sanctions on human altruism', Nature 422, 137-40.

Fitch, W. T. (2007), 'Linguistics: An invisible hand', Nature 449, 665-7. 
Fitch, W. T. and Reby, D. (2001), 'The descended larynx is not uniquely human', Proceedings of the Royal Society 268, 1669-75.

Fox, J., Arena, D. and Bailenson, J. N. (2009), 'Virtual reality: A survival guide for the social scientist', Journal of Media Psychology 21(3), 95-113.

Frank, R. H., Gilovich, T. and Regan, D. T. (1993), 'Does studying economics inhibit cooperation', Journal of Economic Perspectives 7(2), 159-71.

Fresco, A., McGrory, D. and Norfolk, A. (July 6 2006), 'Video of London suicide bomber released', Times Online (Accessed 22 May 2010). http://www. timesonline.co.uk/tol/news/uk/article683824.ece.

Galantucci, B. (2005), 'An experimental study of the emergence of human communication systems', Cognitive Science 29(5), 737-67.

Garrod, S. and Doherty, G. (1994), 'Conversation, co-ordination and convention: an empirical investigation of how groups establish linguistic conventions', Cognition $\mathbf{5 3}(3), 181-215$.

Garrod, S. and Pickering, M. J. (2007), Alignment in dialogue, in M. G. Gaskell and G. Altmann, eds, 'The Oxford Handbook of Psycholinguistics', Oxford University Press, Oxford, pp. 443-52.

Gell-Mann, M. (1992), Complexity and complex adaptive systems, in J. A. Hawkins and M. Gell-Mann, eds, 'The Evolution of Human Languages', Addison-Wesley, New York, pp. 3-18.

Gelman, A. and Stern, H. (2006), "The difference between "significant" and "not significant" is not itself statistically significant', The American Statistician 60(4), 328-31.

Gilbert, N. and Troitzsch, K. G. (2005), Simulation for the Social Scientist, second edn, Open University Press, Maidenhead.

Giles, H., Coupland, N. and Coupland, J. (1991), Accommodation theory: Communication, context and consequence, in H. Giles, J. Coupland and N. Coupland, eds, 'Contexts of Accommodation: Developments in Applied Sociolinguistics', Cambridge University Press, Cambridge, pp. 1-68.

Giles, H., Taylor, D. M. and Bourhis, R. (1973), 'Towards a theory of interpersonal accommodation through language: Some Canadian data', Language in Society 2, 177-92. 
Giles, H. and Wiemann, J. (1987), Language, social comparison, and power, in S. Chaffee and C. R. Berger, eds, 'Handbook of Communication Science', Sage, Beverly Hills, CA, pp. 350-84.

Gintis, H. (2000), 'Strong reciprocity and human sociality', Journal of Theoretical Biology 206(169-79).

Gintis, H., Smith, E. A. and Bowles, S. (2001), 'Costly signaling and cooperation', Journal of Theoretical Biology 213, 103-19.

Gordon, E., Campbell, L., Hay, J., Maclagan, M., Sudbury, A. and Trudgill, P. (2004), New Zealand English: Its Origins and Evolution, Cambridge University Press, Cambridge.

Greenberg, R. D. (2004), Language and Identity in the Balkans: Serbo-Croatian and its Disintegration, Oxford University Press, Oxford.

Hagen, L. K. (2008a), 'The bilingual brain: Human evolution and second language acquisition', Evolutionary Psychology 6(1), 43-63.

Hagen, L. K. (2008b), 'Hagen replies to Hirschfeld (2008)', Evolutionary Psychology 6(1), 186-9.

Haley, K. J. and Fessler, D. M. T. (2005), 'Nobody's watching? subtle cues affect generosity in an anonymous economic game', Evolution and Human Behavior 26(3), 245-56.

Halpin, Z. T. (1991), Kin recognition cues of vertebrates, in P. G. Hepper, ed., 'Kin Recognition', Cambridge University Press, Cambridge, chapter 8, pp. 220-58.

Hamilton, W. D. (1964), 'The genetical evolution of social behaviour, i and ii', Journal of Theoretical Biology 7, 1-52.

Hamilton, W. D. (1996), Narrow Roads of Gene-Land: Evolution of Social Behaviour, Vol. I, W. H. Freeman, Oxford.

Harrington, J. (2006), 'An acoustic analysis of "happy tensing" in the Queen's Christmas broadcasts', Journal of Phonetics 34, 439-57.

Hart, A. J., Whalen, P. J., Shin, L. M., MacInerney, S. C., Fischer, H. and Rauch, S. L. (2000), 'Differential response in the human amygdala to racial outgroup vs ingroup face stimuli', NeuroReport 11(11), 2351-5.

Hauser, M. D., Chomsky, N. and Fitch, W. T. (2002), 'The faculty of language: What is it, who has it, and how did it evolve?', Science 298. 
Henrich, J., Ensminger, J., McElreath, R., Barr, A., Barrett, C., Bolyanatz, A., Cardenas, J. C., Gurven, M., Gwako, E., Henrich, N., Lesorogol, C., Marlowe, F., Tracer, D. and Ziker, J. (2010), 'Markets, religion, community size, and the evolution of fairness and punishment', Science 327, 1480-4.

Hepper, P. G. (2008), 'Kin recognition: Functions and mechanisms. a review', Biological Reviews 61(1), 63-91.

Hepper, P. G., ed. (1991), Kin Recognition, Cambridge University Press, Cambridge.

Herrmann, E., Call, J. and Hernández-Lloreda, M. V. (2007), 'Humans have evolved specialized skills of social cognition: The cultural intelligence hypothesis', Science 317, 1360-6.

Hewes, G. W. (1976), 'The current status of the gestural theory of language origin', Annals of the New York Academy of Sciences 280, 482-504.

Hirschfeld, L. A. (2001), 'On a folk theory of society: Children, evolution, and mental representations of social groups', Personality and Social Psychology Review $5(2), 107-17$.

Hirschfeld, L. A. (2008), 'The bilingual brain revisited: A comment on Hagen (2008)', Evolutionary Psychology 6(1), 182-5.

Hockett, C. F. (1960), 'The origin of speech', Scientific American 203, 88-96.

Hoefler, S. H. and Smith, A. D. M. (2009), 'The pre-linguistic basis of grammaticalisation: A unified approach to metaphor and reanalysis', Studies in Language 33(4), 883-906.

Holmes, J. and Kerswill, P. (2008), 'Contact is not enough: A response to Trudgill', Language in Society $\mathbf{3 7}(2), 273-7$.

Houser, D., Xiao, E., McCabe, K. and Smith, V. (2008), 'When punishment fails: Research on sanctions, intentions and non-cooperation', Games and Economic Behavior 62, 509-32.

Hurley, S. and Chater, N., eds (2005), Perspectives on Imitation, MIT Press, Cambridge, MA.

Hutchinson, J. M. C. and Gigerenzer, G. (2005), 'Simple heuristics and rules of thumn: Where psychologists and behavioural biologists might meet', Behavioural Processes 69, 97-124. 
Ingold, T. (2007), 'The trouble with "evolutionary biology"', Anthropology Today $23(2), 13-7$.

Irons, W. (2001), Religion as a hard-to-fake sign of commitment, in R. M. Nesse, ed., 'Evolution and the Capacity for Commitment', Russell Sage Foundation, New York, pp. 292-309.

Izumi, A. and Kojima, S. (2004), 'Matching vocalizations to vocalizing faces in a chimpanzee (Pan troglodytes)', Animal Cognition 7(179-84).

Jäger, G. (2008), Language evolution and George Price's "general theory of selection", in R. Cooper and R. Kempson, eds, 'Languages in Flux: Dialogue Coordination, Language Variation, Change and Evolution', College Publications, pp. $53-82$.

Jäger, G. (2010), Applications of the price equation to language evolution, in A. D. M. Smith, M. Schouwstra, B. de Boer and K. Smith, eds, 'The Evolution of Language: Proceedings of the 8th International Conference (EVOLANG8)', World Scientific, Singapore, pp. 192-7.

Janda, R. D. and Joseph, B. D. (2003), On language, change, and language changeor, of history, linguistics, and historical linguistics, in B. D. Joseph and R. D. Janda, eds, 'The Handbook of Historical Linguistics', Blackwell, Oxford/Malden, Mass., pp. 3-180.

Joseph, B. D. (1992), Diachronic explanation: Putting speakers back into the picture, in G. W. Davies and G. K. Iverson, eds, 'Explanation in Historical Linguistics', John Benjamins, Amsterdam/Philadelphia, pp. 123-44.

Kalish, M. L., Griffiths, T. L. and Lewandowsky, S. (2007), 'Iterated learning: Intergenerational knowledge transmission reveals inductive biases', Psychonomic Bulletin \& Review 14(2), 288-94.

Keller, L. and Ross, K. G. (1998), 'Selfish genes: A green beard in the red fire ant', Nature 394, 573-5.

Keller, R. (1994), On Language Change: The Invisible Hand in Language, Routledge, London.

Kendon, A. (2002), Historical observations on the relationship between research on sign languages and languages origins theory, in D. F. Armstring, M. A. Karchmer 
and J. V. V. Cleve, eds, 'The Study of Signed Languages: Essays in Honor of William C. Stokoe', Gallaudet University Press, Washington DC, pp. 35-52.

Kendon, A. (2010), The co-involvement of hands and mouth in utterance construction: Implications for language origins theories, in A. D. M. Smith, M. Schouwstra, B. de Boer and K. Smith, eds, 'The Evolution of Language: Proceedings of the 8th International Conference (EVOLANG8)', World Scientific, Singapore, pp. $427-8$.

Kerswill, P. (1996), 'Children, adolescents, and language change', Language Variation and Change 8, 177-202.

Kerswill, P. and Williams, A. (2000), 'Creating a New Town koine: Children and language change in Milton Keynes', Language in Society 29, 65-115.

Kinsella, A. R. (2009), Language Evolution and Syntactic Theory, Cambridge University Press, Cambridge.

Kinzler, K. D., Dupoux, E. and Spelke, E. S. (2007), 'The native language of social cognition', Proceedings of the National Academy of Sciences 104(30), 1257712580 .

Kinzler, K. D., Shutts, K., DeJesus, J. and Spelke, E. S. (2009), 'Accent trumps race in guiding children's social preferences', Social Cognition 27(4), 623-34.

Kirby, J. P. (to appear), The role of probabilistic enhancement in phonologization, in A. Yu, ed., 'Origin of Sound Patterns: Approaches to Phonologization', Oxford University Press, Oxford.

Kirby, S. (1999), Function, Selection and Innateness: The Emergence of Language Universals, Oxford University Press, Oxford.

Kirby, S. (2002), 'Natural language from artificial life', Artificial Life 8, 185-215.

Kirby, S., Cornish, H. and Smith, K. (2008), 'Cumulative cultural evolution in the laboratory: An experimental approach to the origins of structure in human language', Proceedings of the National Academy of Sciences 105(31), 10681-6.

Kirby, S., Dowman, M. and Griffiths, T. L. (2007), 'Innateness and culture in the evolution of language', Proceedings of the National Academy of Sciences 104(12), 5241-5.

Kirby, S., Smith, K. and Brighton, H. (2004), 'From UG to universals: Linguistic adaptation through iterated learning', Studies in Language 28(5), 587-607. 
Kirby, T. (1 April 2006), 'Voice from Jamaica divides Radio 4 listeners', The Independent Online (Accessed 22 May 2010). http://www.independent.co.uk/news/ media/voice-from-jamaica-divides-radio-4-listeners-472301.html.

Klemens, J. A. (2008), 'Kin recognition in plants?', Biology Letters 4, 67-8.

Kokko, H., Johnstone, R. A. and Clutton-Brock, T. H. (2001), 'The evolution of cooperative breeding through group augmentation', Proceedings of the Royal Society 268, 187-96.

Komdeur, J. and Hatchwell, B. J. (1999), 'Kin recognition: Function and mechanisms in avian societies', Trends in Ecology and Evolution 14, 237-41.

Komdeur, J., Richardson, D. S. and Hatchwell, B. (2008), Kin-recognition mechanisms in cooperative breeding systems: Ecological causes and behavioral consequences of variation, in J. Korb and J. Heinze, eds, 'Ecology of Social Evolution', Springer-Verlag, Berlin, chapter 8, pp. 175-93.

Krackhardt, D. and Stern, R. N. (1988), 'Informal networks and organizational crises: An experimental simulation', Social Psychology Quarterly 51(2), 123-40.

Kroch, A. S. (1978), 'Toward a theory of social dialect variation', Language in Society 7, 17-36.

Labov, W. (1963), 'The social motivation of a sound change', Word 19, 273-309.

Labov, W. (1982), Building on empirical foundations, in W. P. Lehmann and Y. Malkiel, eds, 'Perspectives on Historical Linguistics', John Benjamins, Amsterdam, pp. 17-92.

Labov, W. (2001), Principles of Linguistic Change. Volume 2: Social Factors, Blackwell, Malden, MA.

Laham, S. M., Gonsalkorale, K. and von Hippel, W. (2005), 'Darwinian grandparenting: Preferential investment in more certain kin', Personality and Social Psychology Bulletin 31(1), 63-72.

Lakin, J. L. and Chartrand, T. L. (2003), 'Using nonconscious behavioral mimicry to create affiliation and rapport', Psychological Science 14, 334-9.

Lakin, J. L., Chartrand, T. L. and Arkin, R. M. (2008), 'I am too just like you: Nonconscious mimicry as an automatic behavioral response to social exclusion', Psychological Science 19(8), 816-22. 
Laland, K. N. (2002), Gene-culture co-evolution, in L. Nadel, ed., 'Encyclopedia of Cognitive Science', John Wiley \& Sons, pp. 268-74.

Laland, K. N. (2008), 'Exploring gene-culture interactions: Insights from handedness, sexual selection and niche-construction case studies', Philsophical Transactions of the Royal Society B 363, 3577-89.

Lass, R. (1990), 'How to do things with junk: Exaptation in language change', Journal of Linguistics 26(1), 79-102.

Lass, R. (1997), Historical Linguistics and Language Change, Cambridge University Press, Cambridge.

Lehmann, L., Keller, L., West, S. and Roze, D. (2007), 'Group selection and kin selection: Two concepts but one process', Proceedings of the National Academy of Sciences 104(16), 6736-9.

Lenneberg, E. (1967), Biological Foundations of Language, Wiley, New York.

Levelt, W. J. M. (2004), 'Speech, gesture and the origins of language', European Review 12(4), 543-9.

Lindeman, R. L. (1942), 'Experimental simulation of winter anaerobiosis in a senescent lake', Ecology 23(1), 1-13.

Lindgren, J. R. and Pegalis, L. (1989), 'Non-kin adoption and sociobiology', Journal of Social and Biological Structures 12, 83-6.

Livingstone, D. (2002), The evolution of dialect diversity, in A. Cangelosi and D. Parisi, eds, 'Simulating the Evolution of Language', Springer-Verlag, London, pp. 99-117.

Livingstone, D. and Fyfe, C. (1999), Modelling the evolution of dialect diversity, in D. Floreano, J.-D. Nicoud and F. Mondada, eds, 'Advances in Artificial Life, Proceedings of the 5th European Conference in Artificial Life, ECAL '99, Laussane, Switzerland', Springer-Verlag, London, pp. 704-8.

LoCastro, V. (2001), 'Individual differences in second language acquisition: Attitudes, learner subjectivity and L2 pragmatic norms', System 29(1), 69-89.

Lupyan, G. and Dale, R. (2010), 'Language structure is partly determined by social structure', PLoS ONE 5(1), e8559. 
Maddieson, I. (2008), Uvular consonants, in M. Haspelmath, M. S. Dryer, D. Gil and B. Comrie, eds, 'The World Atlas of Language Structures Online', Max Planck Digital Library, Munich, chapter 6.

Martindale, C. (1973), 'An experimental simulation of literary change', Journal of Personality and Social Psychology 25(3), 319-26.

Maynard Smith, J. and Harper, D. G. C. (2003), Animal Signals, Oxford University Press, Oxford.

Maynard Smith, J. and Szathmáry, E. (1995), The Major Transitions in Evolution, Oxford University Press, Oxford.

McElreath, R., Boyd, R. and Richerson, P. J. (2003), 'Shared norms and the evolution of ethnic markers', Current Anthropology 44(1), 122-9.

McMahon, A. M. S. (1994), Understanding Language Change, Cambridge University Press, Cambridge.

McMahon, A. and McMahon, R. (2005), Language Classification by Numbers, Oxford University Press, Oxford.

Mendlowitz, M. and Leslie, A. (1942), 'The experimental simulation in the dog of the cyanosis and hypertrophic osteoarthropathy which are associated with congenital heart disease', American Heart Journal 42(2), 141-52.

Mesoudi, A. and O'Brien, M. (2008), 'The cultural transmission of Great Basin projectile-point technology I: An experimental simulation', American Antiquity 73(1), 3-28.

Mesoudi, A., Whiten, A. and Laland, K. N. (2004), 'Is human cultural evolution Darwinian? evidence reviewed from the perspective of the Origin of Species', Evolution 58(1), 1-11.

Mesoudi, A., Whiten, A. and Laland, K. N. (2006), 'Towards a unified science of cultural evolution', Behavioral and Brain Sciences 29(4), 329-83.

Meyerhoff, M. (1998), 'Accommodating your data: The use and misuse of accommodation theory in sociolinguistics', Language and Communication 18, 205-25.

Meyerhoff, M. (2006), Introducing Sociolinguistics, Routledge, Oxon/New York.

Miall, C. E. (1987), 'The stigma of adoptive parent status: Perceptions of community attitudes toward adoption and the experience of informal social sanctioning', Family Relations 36(1), 34-9. 
Michalski, R. L. and Shackelford, T. K. (2005), 'Grandparental investment as a function of relational uncertainty and emotional closeness with parents', Human Nature 16(3), 293-305.

Miller, G. F. (2001), The Mating Mind: How Sexual Choice Shaped the Evolution of Human Nature, Vintage, London.

Miller, J. L. (1994), 'On the internal structure of phonetic categories: A progress report', Cognition 50, 271-85.

Milroy, J. (1992), A social model for the interpretation of language change, in M. Rissanen, O. Ihalainen, T. Nevalainen and I. Taavitsainen, eds, 'History of Englishes: New Methods and Interpretation in Historical Linguistics', Mouton de Gruyter, Berlin, pp. 72-91.

Milroy, J. and Milroy, L. (1985), 'Linguistic change, social network and speaker innovation', Journal of Linguistics 21, 339-84.

Moon, C., Cooper, R. P. and Fifer, W. P. (1993), 'Two-day-olds prefer their native language', Infant Behavior and Development 16, 495-500.

Moore, E. (2003), Learning Style and Identity: A Sociolinguistic Analysis of a Bolton High School, PhD thesis, University of Manchester, UK.

Moore, E. (2004), 'Sociolinguistic style: A multidimensional resource for shared identity creation', Canadian Journal of Linguistics 49(3/4), 375-96.

Morowitz, H. J. and Singer, J. L., eds (1994), The Mind, The Brain, and Complex Adaptive Systems, Addison-Wesley, New York.

Mufwene, S. S. (2001), The Ecology of Language Evolution, Cambridge University Press, Cambridge.

Mufwene, S. S. (2006), 'The comparability of new-dialect formation and creoledevelopment', World Englishes 25, 177-86.

Mufwene, S. S. (2008), 'Colonization, population contacts, and the emergence of new language varities: A response to Peter Trudgill', Language in Society $37(2), 254-8$.

Nettle, D. (1999), Linguistic Diversity, Oxford University Press, Oxford.

Nettle, D. and Dunbar, R. I. M. (1997), 'Social markers and the evolution of cooperative exchange', Current Anthropology 38(1), 93-9. 
Neville, H. J., Mills, D. L. and Lawson, D. S. (1992), 'Fractionating language: Different neural subsystems with different sensitive periods', Cerental Cortex 2(3), 24458.

Newport, E., Bavelier, D. and Neville, H. (2001), Critical thinking about critical periods, in D. Dupoux, ed., 'Language, Brain, and Cognitive Development: Essays in Honor of Jacques Mehler', MIT Press, Cambridge, MA.

Nieto-Sampedro, M. and Nieto-Diáz, M. (2005), 'Neural plasticity: Changes with age', Journal of Neural Transmission 112, 3-27.

Norris, D. A. (1993), Teach Yourself Serbo-Croat, Hodder and Stoughton, London.

Norris, D. A. (2003), Teach Yourself Croation, Hodder Headline, London.

Nottebohm, F. (1972), 'The origins of vocal learning', The American Naturalist 106(947), 116-40.

Nowak, M. A. and Sigmund, K. (1998a), 'The dynamics of indirect reciprocity', Journal of Theoretical Biology 194, 561-74.

Nowak, M. A. and Sigmund, K. (1998b), 'Evolution of indirect reciprocity by image scoring', Nature 393, 573-7.

O'Brien, M. J. (2006), 'Archaeology and cultural macroevolution.', Behavioral and Brain Sciences 29(4), 359-60.

Ohala, J. J. (1990), The phonetics and phonology of aspects of assimilation, in J. Kingston and M. Beckman, eds, 'Papers in Laboratory Phonology I: Between the Grammar and the Physics of Speech', Cambridge University Press, pp. 25875.

Oudeyer, P.-Y. (2005), 'How phonological structures can be culturally selected for learnability', Adaptive Behavior 13(4), 269-80.

Oyama, S. (1978), 'The sensitive period and comprehension of speech', The Journal for the National Association for Bilingual Education 3(1), 25-39.

Panchanathan, K. and Boyd, R. (2003), 'A tale of two defectors: The importance of standing for evolution of indirect reciprocity', Journal of Theoretical Biology 224, 115-26.

Pardo, J. S. (2006), 'On phonetic convergence during conversational interaction', Journal of the Acoustic Society of America 119(4), 2382-93. 
Patkowski, M. S. (1980), 'The sensitive period for the acquisition of syntax in a second language', Language Learning 30, 449-72.

Penfield, W. and Roberts, L. (1959), Speech and Brain Mechanisms, Princeton University Press, Princeton, NJ.

Petitto, L. A. and Holowka, S. (2002), 'Evaluating attributions of delay and confusion in young bilinguals: Special insights from infants acquiring a signed and a spoken language', Sign Language Studies 3(1), 4-33.

Pickering, M. and Garrod, S. (2004), 'Towards a mechanistic psychology of dialogue', Behavioral and Brain Sciences 27, 169-226.

Pickering, M. J. and Garrod, S. (2006), 'Alignment as the basis for successful communication', Research on Language and Computation 4, 203-228.

Pierrehumbert, J. (2001), Exemplar dynamics: Word frequency, lenition, and contrast, in J. L. Bybee and P. Hopper, eds, 'Frequency Effects and the Emergence of Linguistic Structure', John Benjamins, Amsterdam, pp. 137-57.

Pinker, S. and Bloom, P. (1990), 'Natural language and natural selection', Behavioral and Brain Sciences 13, 707-84.

Pope, J., Meyerhoff, M. and Ladd, D. R. (2007), 'Forty years of language change on Martha's Vineyard', Language 83(3), 615-27.

Price, G. R. (1970), 'Selection and covariance', Nature 227(5257), 520-1.

Price, G. R. (1972), 'Extension of covariance selection mathematics', Annals of Human Genetics 36(2), 129-40.

Price, G. R. (1995), 'The nature of selection', Journal of Theoretical Biology $\mathbf{1 7 5}(3), 373-88$.

Quinn, M. (2001), Evolving communication without dedicated communication channels, in J. Kelemen and P. Sosik, eds, 'Advances in Artificial Life. Proceedings of the 6th European Conference on Artificial Life, ECAL 2001', Springer-Verlag, London, pp. 357-66.

Rendall, D., Rodman, P. S. and Emond, R. E. (1996), 'Vocal recognition of individuals and kin in free-ranging rhesus monkeys', Animal Behaviour 51, 1007-15.

Richerson, P. J., Boyd, R. T. and Henrich, J. (2003), Cultural evolution of human cooperation, in P. Hammerstein, ed., 'Genetic and Cultural Evolution of Cooperation', MIT Press, Cambridge, MA, chapter 19, pp. 357-88. 
Ritt, N. (2004), Selfish Sounds and Linguistic Evolution: A Darwinian Approach to Language Change, Cambridge University Press, Cambridge.

Roberts, G. (2008), 'Language and the freerider problem: an experimental paradigm', Biological Theory 3(2), 174-83.

Roberts, G. (2010), 'An experimental study of the role of social selection and frequency of interaction in linguistic diversity', Interaction Studies 11(10), 138-59.

Ruffle, B. J. and Sosis, R. (2007), 'Does it pay to pray? Costly ritual and cooperation', B.E. Journal of Economic Analysis and Policy 7, 1-35.

Sampson, G. (2005), The "Language Instinct" Debate, 2nd edn, Continuum, London/New York.

Schneider, E. W. (2008), 'Accommodation versus identity? A response to Trudgill', Language in Society 37(2), 262-67.

Scott-Phillips, T. (2008), 'Defining biological communication', Journal of Evolutionary Biology 21, 387-95.

Scott-Phillips, T., Kirby, S. and Ritchie, G. R. S. (2009), 'Signalling signalhood and the emergence of communication', Cognition 113, 226-33.

Scotton, C. M. (1985), What the heck, sir: Style shifting and lexical colouring as features of powerful language, in R. L. Street, Jr. and J. N. Cappella, eds, 'Sequence and Pattern in Communicative Behaviour', Edward Arnold, London, pp. 103-19.

Sears, D. O. (1986), 'College sophomores in the laboratory: Influences of a narrow data base on social psychology's view of human nature', Journal of Personality and Social Psychology 51(3), 515-30.

Seltig, M. (1985), 'Levels of style-shifting exemplified in the interaction strategies of a moderator in a listener participation programme', Journal of Pragmatics 9, 179-97.

Sharp, S. P., McGowan, A., Wood, M. J. and Hatchwell, B. J. (2005), 'Learned kin recognition cues in a social bird', Nature 434, 1127-30.

Shepher, J. (1971), 'Mate selection among second generation kibbutz adolescents and adults: Incest avoidance and negative imprinting', Archives of Sexual Behavior 1(4), 293-307. 
Sluckin, W. (1973), Imprinting and Early learning, second edn, Transaction Publishers, New Jersey.

Soltis, J., Boyd, R. and Richerson, P. J. (1995), 'Can group-functional behaviors evolve by cultural group selection', Current Anthropology 36(3), 473-94.

Sosis, R. (2003), 'Why aren't we all Hutterites: Costly signaling theory and religious behavior', Human Nature 14(2), 91-127.

Sperber, D. (2000), An objection to the memetic approach to culture, in R. Aunger, ed., 'Darwinizing Culture: The Status of Memetics as a Science', Oxford University Press, Oxford, pp. 167-73.

Steels, L. (2000), Language as a complex adaptive system, in M. Schoenauer, K. Deb, G. Rudolph, X. Yao and E. Lutton, eds, 'Proceedings of the 6th International Conference on Parallel Problem Solving from Nature', Springer-Verlag, Berlin, pp. 17-26.

Taylor, P. D. (1992a), 'Altruism in viscous populations - an inclusive fitness model', Evolutionary Ecology 6, 654-74.

Taylor, P. D. (1992b), 'Inclusive fitness in a homogeneous environment', Proceedings of the Royal Society 249, 299-302.

Tomasello, M. (2008), Origins of Human Communication, MIT Press, Cambridge, MA.

Traulsen, A. and Nowak, M. A. (2006), 'Evolution of cooperation by multilevel selection', Proceedings of the National Academy of Sciences 103(29), 10952-5.

Trivers, R. L. (1971), 'The evolution of reciprocal altruism', The Quarterly Review of Biology 46, 35-57.

Trudgill, P. (1974), The Social Differentiation of English in Norwich, Cambridge University Press, Cambridge.

Trudgill, P. (1986), Dialects in Contact, Blackwell, Oxford.

Trudgill, P. (2004), New-Dialect Formation: The Inevitability of Colonial Englishes, Edinburgh University Press, Edinburgh.

Trudgill, P. (2008a), 'Colonial dialect contact in the history of European languages: On the irrelevance of identity to new-dialect formation', Language in Society $37(2), 241-54$. 
Trudgill, P. (2008b), 'On the role of children, and the mechanical view: A rejoinder', Language in Society $\mathbf{3 7}(2)$, 277-80.

Tuten, D. N. (2008), 'Identity formation and accommodation: Sequential and simultaneous relations', Language in Society 37(2), 259-62.

Vogt, P. (2000), 'Bootstrapping grounded symbols by minimal autonomous robots', Evolution of Communication 4(1), 89-118.

Volterra, V., Caselli, M. C., Capirci, O. and Pizzuto, E. (2004), Gesture and the emergence and development of language, in M. Tomasello and D. I. Slobin, eds, 'Beyond Nature-Nurture: Essays in Honor of Elizabeth Bates', Lawrence Erlbaum Associates, New Jersey, pp. 3-40.

Waters, J. F. and Glass, R. E. (1970), Penetration of sonic boom energy into the ocean: an experimental simulation, Technical report, Department of Transportation, Federal Aviation Administration, Office of Noise Abatemant.

Webster, N. (1789), Dissertations on the English Language, Isaiah Thomas, Boston.

Wedel, A. B. (2006), 'Exemplar models, evolution and language change', The Linguistic Review 23, 247-74.

Weinreich, U., Labov, W. and Herzog, M. I. (1968), Empirical foundations for a theory of language change, in W. P. Lehmann and Y. Malkiel, eds, 'Directions for Historical Linguistics: A Symposium', University of Texas Press, Austin/London, pp. $95-188$.

West, S. A. and Gardner, A. (2010), 'Altruism, spite, and greenbeards', Science 327, 1341-4.

West, S. A., Griffin, A. S. and Gardner, A. (2007), 'Social semantics: Altruism, cooperation, mutualism, strong reciprocity and group selection', Journal of Evolutionary Biology 20(2), 415-32.

Whallon, R. (2006), "Social networks and information: Non-"utilitarian" mobility among hunter-gatherers', Journal of Anthropological Archaeology 25, 259-70.

Whinnom, K. (1971), Linguistic hybridization and the 'special case' of pidgins and creoles, in D. Hymes, ed., 'Pidginization and Creolization of Languages', Cambridge University Press, Cambridge, pp. 91-115. 
Williams, K. D., Cheung, C. K. T. and Choi, W. (2000), 'Cyberostracism: Effects of being ignored over the internet', Journal of Personality and Social Psychology 79(5), 748-62.

Williams, K. D., Forgas, J. P. and von Hippel, W., eds (2005), The Social Outcast: Ostracism, Social Exclusion, Rejection, and Bullying, Psychology Press, New York.

Willis, R. H. and Long, N. J. (2007), 'An experimental simulation of an internation truel', Behavioral Science 12(1), 24-32.

Wilson, D. S. (1975), 'A theory of group selection', Proceedings of the National Academy of Sciences 72, 143-6.

Wilson, D. S. (1977), 'Structured demes and the evolution of group-advantageous traits', American Naturalist 111, 157-85.

Wilson, D. S. (2007), 'Social semantics: Toward a genuine pluralism in the study of social behavior', Journal of Evolutionary Biology 21, 368-73.

Wilson, D. S., Pollock, G. B. and Dugatkin, L. A. (1992), 'Can altruism develop in a purely viscous population', Evolutionary Ecology 6, 331-41.

Wray, A. and Grace, G. W. (2007), 'The consequences of talking to strangers: Evolutionary corollaries of socio-cultural influences on linguistic form', Lingua $117,543-78$.

Yamauchi, H. (2004), Baldwinian Accounts of Language Evolution, PhD thesis, University of Edinburgh, Edinburgh.

Zahavi, A. (1975), 'Mate selection - a selection for a handicap', Journal of Theoretical Biology 53, 205-14.

Zahavi, A. (1977), 'The cost of honesty (further remarks on the handicap principle)', Journal of Theoretical Biology 67, 603-5.

Zahavi, A. and Zahavi, A. (1997), The Handicap Principle: A Missing Piece of Darwin's Puzzle, Oxford University Press, Oxford.

Zak, P. J., Stanton, A. A. and Ahmadi, S. (2007), 'Oxytocin increases generosity in humans', PLoS ONE 2(11), e1128. 